\title{
DOMINATION NUMBER WITHIN ON-LINE SOCIAL NETWORKS
}

\author{
by \\ Marc Lozier \\ Bachelor of Engineering in Computer Engineering, \\ Ryerson University, 2013
}

A thesis presented to Ryerson University

in partial fulfillment of the requirements for the degree of

Master of Science

in the Program of

Applied Mathematics

Toronto, Ontario, Canada, 2015

(c) Marc Lozier 2015 


\section{Author's Declaration}

I hereby declare that I am the sole author of this thesis. This is a true copy of the thesis, including any required final revisions, as accepted by my examiners.

I authorize Ryerson University to lend this thesis to other institutions or individuals for the purpose of scholarly research.

I further authorize Ryerson University to reproduce this thesis by photocopying or by other means, in total or in part, at the request of other institutions or individuals for the purpose of scholarly research.

I understand that my thesis may be made electronically available to the public. 


\title{
Domination Number within On-line Social Networks \\ Master of Science, 2015 \\ Marc Lozier \\ Applied Mathematics, Ryerson University
}

\begin{abstract}
There is particular interest in on-line social networks (OSNs) and capturing their properties. The memoryless geometric protean (MGEO-P) model provably simulated many OSN properties. We investigated dominating sets in OSNs and their models. The domination numbers were computed using two algorithms, DS-DC and DS-RAI, for MGEO-P samples and Facebook data, known as the Facebook 100 graphs. We establish sub-linear bounds on the domination numbers for the Facebook 100 graphs, and show that these bounds correlate well with bounds in graphs simulated by MGEO-P.

A new model is introduced known as the Distance MGEO-P (DMGEO-P) model. This model incorporates geometric distance to influence the probability that two nodes are adjacent. Domination number upper bounds were found to be well-correlated with the Facebook 100 graphs.
\end{abstract}




\section{Acknowledgements}

I would first like to take this opportunity to extend my thanks and obligation to those people whose help and active guidance were critical in the completion of the thesis.

I am foremost indebted to my supervisor Dr. Anthony Bonato for the wealth of knowledge he has provided. His support and guidance has been invaluable to both my research and future endeavors.

I would like to offer my profound thanks to Dr. Dejan Delic and Dr. Laleh Samarbaksh for their participation as my defense committee. I would also like to thank Dr. Sebastian Ferrando for encouraging me to pursuit my Masters in Applied Mathematics. Collectively, I would like to thank the entire Department of Mathematics at Ryerson University for the great experience as a Masters student and Graduate Assistant.

Lastly, I would like to thank my family — my mother and father Judy and Michel, and my brothers Matthew and Michael — and my friends for their support, encouragement and understanding. 


\section{Contents}

List of Figures vii

$\begin{array}{ll}\text { Chapter 1. Introduction } & 1\end{array}$

1.1. Motivation 1

1.2. Graph Theory 2

1.3. Properties of On-line Social Networks 6

$\begin{array}{ll}\text { 1.4. Probability } & 14\end{array}$

1.5. Dominating On-line Social Networks 19

1.6. Outline of Thesis 21

Chapter 2. Memoryless Geometric Protean Model 23

2.1. Background 23

2.2. The Model 24

$\begin{array}{ll}\text { 2.3. Simulations } & 25\end{array}$

Chapter 3. Domination Number in Facebook 100 and the MGEO-P Model 31

3.1. Facebook $100 \quad 31$

3.2. Domination Number within Facebook 100

3.3. Domination Number within MGEO-P 34

Chapter 4. Distance Memoryless Geometric Protean Model 39 
4.2. The Model 40

4.3. Domination Number of the DMGEO-P Model 45

Chapter 5. Conclusions and Future Directions 49

5.1. Summary 49

5.2. Open Problems 50

Appendix A. Data Tables 53

A.1. MGEO-P Data Tables 53

A.2. Facebook 100 Data Tables 68

A.3. DMGEO-P Data Tables 81

$\begin{array}{lll}\text { Appendix B. Program Code } & 91\end{array}$

B.1. JUNG Code (Java) 91

$\begin{array}{lll}\text { B.2. } & \text { SNAP Code }(\mathrm{C}++) & 164\end{array}$

$\begin{array}{ll}\text { Bibliography } & 219\end{array}$ 


\section{List of Figures}

1.1 An example of a simple graph.

1.2 A graph and one of its dominating sets (represented by the white vertices).

1.3 The 1-core, 2-core and 3-core of a random graph. 5

1.4 Dominating sets generated by the algorithms DS-DC and DS-RAI. 7

1.5 General shape of a power-law distribution. 11

1.6 A log-log plot for Facebook friendships [43]. 11

1.7 Log-log plots for Flickr, LiveJournal, Orkut and Youtube demonstrating the existence of power law degree distribution [35]. 12

1.8 Sample graph from $G(n, p)$ where $n=10$ and $p=0.5$. 17

$\begin{array}{lll}1.9 & \text { General shape of a binomial distribution. } & 18\end{array}$

2.1 Time steps of a MGEO-P(3,2,0.17, 0.27) sample. 26

2.2 Samples of the $\operatorname{MGEO-P}(n, m, \alpha, \beta, p)$ model, with various choices of parameters.

2.3 Degree distribution of $\operatorname{MGEO}-\mathrm{P}(n, 4,0.17,0.27,1)$ samples and $\mathrm{G}(n, p)$ model.

2.4 Log-log plots of $\operatorname{MGEO-P}(n, 4,0.17,0.27,1)$ samples and $\mathrm{G}(n, p)$ model. 
3.1 Domination number upper bounds of the Facebook 100 graphs.

3.2 Domination number upper bounds of the Facebook 100 graphs with Theorem 1.1 upper bound $(\delta=5)$, presenting a significant overestimation.

3.3 Domination number upper bounds of MGEO-P samples corresponding to the Facebook 100 graphs.

3.4 Domination number upper bounds of MGEO-P samples corresponding to the Facebook 100 graphs with Theorem 1.1 upper bound $(\delta=5)$.

3.5 Domination number upper bounds of the Facebook 100 graphs with Theorem 3.1 upper bound, showing a very close correlation.

4.1 Time steps of a DMGEO-P $(3,2,0.17,0.27)$ sample.

4.2 Samples of the DMGEO-P $(n, m, \alpha, \beta)$ model, with various choices of parameters.

4.3 Degree distribution of $\operatorname{DMGEO-P}(n, 4,0.17,0.27)$ samples for $n=1,000,5,000,10,000$ and 50,000.

4.4 Log-log plots of $\operatorname{DMGEO-P}(n, 4,0.17,0.27)$ samples for $n=1,000,5,000,10,000$ and 50,000.

4.5 Domination number upper bounds of DMGEO-P samples corresponding to the Facebook 100 graphs using the DS-RAI algorithm. 
4.6 Domination number upper bounds of DMGEO-P samples corresponding to the Facebook 100 graphs and FB100.

4.7 Domination number upper bounds of DMGEO-P samples corresponding to the Facebook 100 graphs with Theorem 1.1 upper bound $(\delta=5)$.

4.8 Domination number upper bounds of DMGEO-P samples corresponding to the Facebook 100 graphs with Theorem 1.1 upper bound $(\delta=5)$. 



\section{CHAPTER 1}

\section{Introduction}

\subsection{Motivation}

Over the last decade, research on on-line social networks (or OSNs), such as Facebook and Twitter, has been increasing within the network science community. There is particular interest in these networks and capturing their properties. A few of the commonly observed properties of OSNs and other complex networks are power-law degree distributions [5] and the small world property [44] (constant or shrinking diameter with network size and high local clustering) [25]. These properties will be defined in Section 1.3. Graph theorists have the challenge to come up with a model that can mimic these properties asymptotically with high probability, and which correlate well with real-world data sets. Modelling allows mathematicians to uncover the underlying mechanisms behind the network, predict its future, and reveal any hidden reality of the network; see $[\mathbf{8}]$.

Graphs capture relationships between objects. A graph is called a network when it is represented by real-world interactions, such as the web graph, biological networks, or social networks. The friendship graph, collaboration graph and actor graphs are examples of social networks. These graphs are structured differently when compared to the web graph: the web graph is organized by link structure, while social networks are governed by social 
interactions between people. On-line networks have their data more readily accessible and measurable than offline social networks (where the data is noisier and harder to gather). Hence, there is an increasing interest for a rigorous model to capture OSN's evolutionary properties. However, some models are more difficult to analyze than others. While some may fit naturally and realistically with real world data, they are often challenging to analyze.

\subsection{Graph Theory}

Some knowledge of graph theory is necessary before discussing OSNs and their models. A graph $G$ consists of a non-empty set of vertices, denoted $E(G)$, and an edge set, denoted $E(G)$, which consists of pairs of vertices. A graph is directed if each edge has an orientation. Otherwise, it is known as an undirected graph. When two nodes are joined by an edge, they are adjacent to each other or called neighbours. For simplicity, we will only consider simple graphs (see Figure 1.1): graphs that do not contain loops or multiple edges. The order of a graph is its number of vertices (that is, $|V(G)|$ ), while its size is the number of edges (that is, $|E(G)|$ ). In OSNs, vertices correspond to people, and edges correspond to their friendship relationships.

A subgraph of graph $G$ is a graph $H$ where $V(H) \subseteq V(G)$ and $E(H) \subseteq$ $E(G)$. An induced subgraph of graph $G$ is a graph $S$, where $V(S) \subseteq V(G)$ and each vertex is adjacent to each other only if they are adjacent in $G$. A path is said to exist between two distinct vertices when there is a sequence of edges that connect a sequence of non-repeated vertices to them. A graph is 
connected when for each pair of distinct vertices, there exist a path between them.

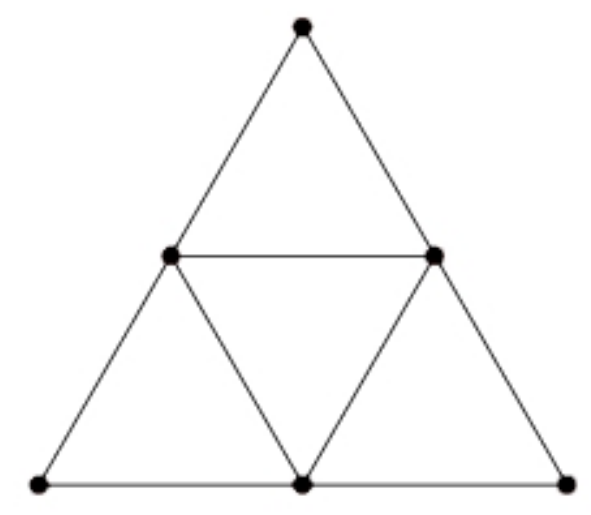

Figure 1.1. An example of a simple graph.

The neighbour set of a given vertex $u$, denoted by $N(u)$, consists of the set of vertices adjacent to $u$. The degree of a vertex, written $\operatorname{deg}(u)$, is the cardinality of its neighbour set $|N(u)|$. The minimum degree of a graph $G$ is denoted as $\delta(G)$. A familiar global measure for distances in a graph is its diameter. The diameter of a graph $G$, written $\operatorname{diam}(G)$, is the length of the longest shortest path. The distance between two vertices is the length of the shortest path between them, known as $d(u, v)$.

1.2.1. Dominating Sets. Dominating sets in graphs were first introduced by Ore in 1962 [37]. A dominating set $S \subseteq V(G)$ has the property that each vertex not in $S$ is adjacent to some vertex in $S$. The domination number, written $\gamma(G)$, is the minimal cardinality of a dominating set. It is difficult to compute the exact value of $\gamma(G)$ when given a large order graph $G$. In particular, computing the domination number is known to be a $\mathbf{N P}$-complete problem [19]. In order to obtain a value for $\gamma(G)$, heuristic algorithms are 
used. One proof for the upper bounds of the domination number of any given graph was given using the probabilistic method by Alon and Spencer in [3], stated as Theorem 1.1. Figure 1.2 shows an example of a dominating set.

THEOREM 1.1. [3] If given any graph $G$ with a minimum degree $\delta>1$, then

$$
\gamma(G) \leq n\left(\frac{1+\log (\delta+1)}{\delta+1}\right)
$$

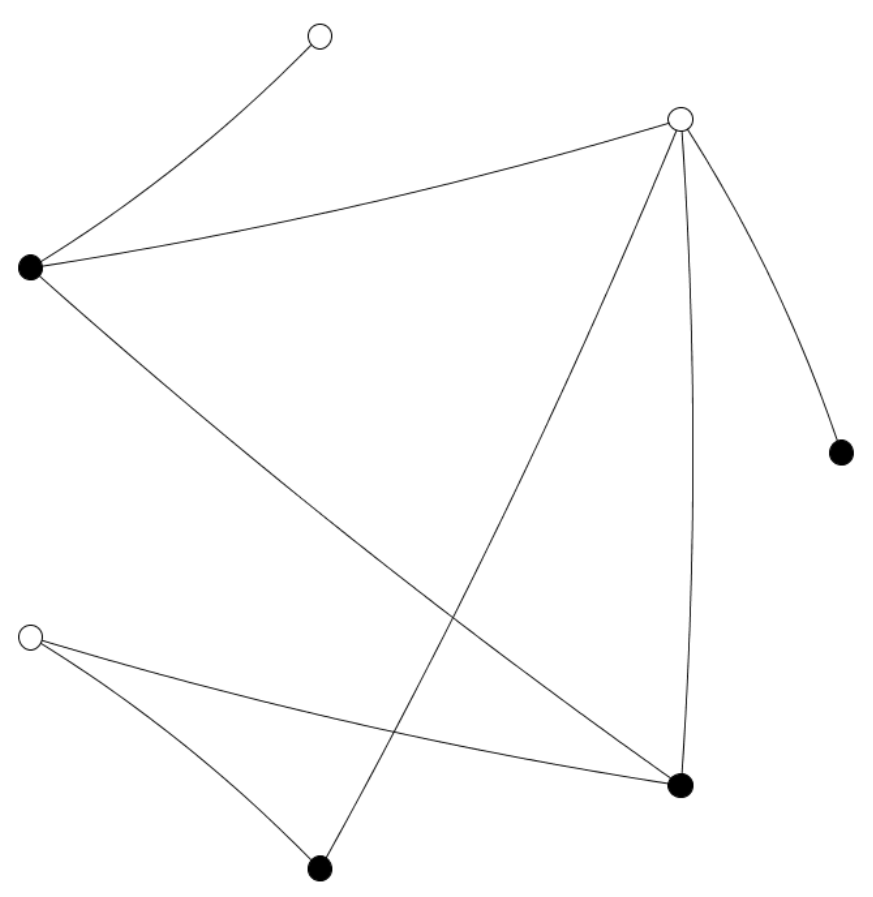

Figure 1.2. A graph and one of its dominating sets (represented by the white vertices).

The domination set is quite sensitive to vertices with low degrees. During simulations, the $k$-cores (where $1 \leq k \leq 5$ ) for the graphs were used to test 
how sensitive their domination sets were. The $k$-core of a graph is the largest induced subgraph in which its minimum degree is at least $k$. Figure 1.3 shows an example of the 1-, 2-, and 3-core of a graph.

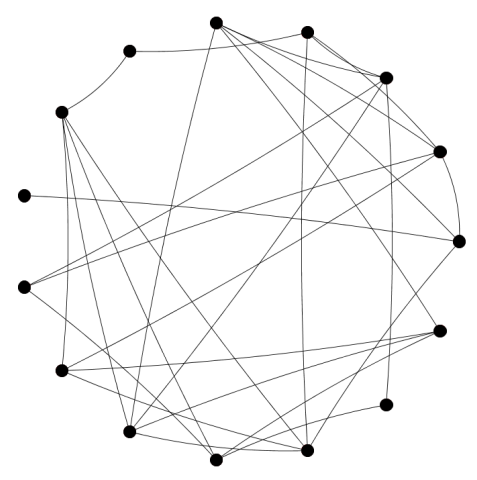

1-core

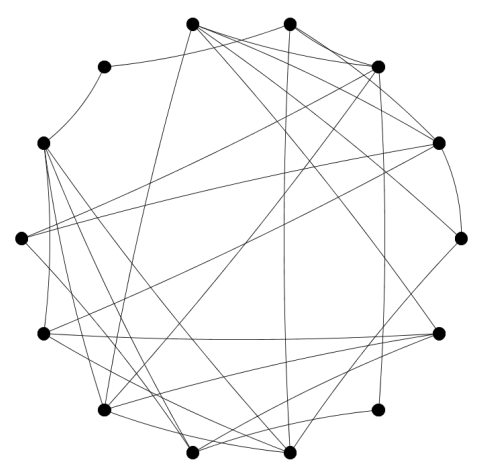

2-core

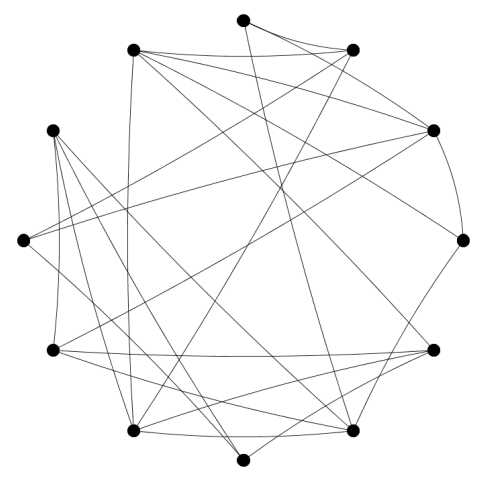

3-core

Figure 1.3. The 1-core, 2-core and 3-core of a random graph.

There were two main algorithms used to provide results. Additional algorithms were used but not described in detailed in the thesis (as they were outperformed by other algorithms). Some of these include DS-RANDOM (adding random vertices to the dominating set), DS-GREEDY (adding the highest degree vertices to the dominating set recursively until entire graph is covered), and DS-RANK (adding vertices with the highest rank recursively, MGEO-P model only).

The first main algorithm used in the thesis is known as DS-RAI. It is the fastest performing algorithm based on heuristic evidence from our experiments and provides a good upper bound (but not the best in our experiments). The algorithm works as described in Algorithm 1 (where \{\} denotes an empty set). The second main algorithm used is known as DS-DC. Despite 
INPUT: Graph data - set of nodes V with degrees;

OUTPUT: Dominating set array - DS;

initialization: $\mathrm{DS}=\{\}$, coveredSet $=\{\}$, sort nodes $\mathrm{V}$ in descending order by degree;

while not at end of $V$ do

$\mathrm{v} \in \mathrm{V}$;

if $v$ is not in coveredSet then

add $\mathrm{v}$ to DS;

add $\mathrm{v}$ to coveredSet;

add neighbours of $\mathrm{v}$ to coveredSet;

end

end

Algorithm 1: Dominating set algorithm DS-RAI from [39].

it performing slower than DS-RAI, it provides a better upper bound. The algorithm is stated in Algorithm 2.

INPUT: Graph data - set of nodes V with degrees;

OUTPUT: Dominating set array - DS;

initialization: $\mathrm{DS}=\mathrm{V}$, sort nodes $\mathrm{V}$ in ascending order by degree;

while not at end of $V$ do

$\mathrm{v} \in \mathrm{V}$;

remove $\mathrm{v}$ from DS;

if $D S$ is not dominating then

add v to DS;

end

end

Algorithm 2: Dominating set algorithm DS-DC from [33].

\subsection{Properties of On-line Social Networks}

Emergent topological properties have been observed with the available real world data from large-scale on-line social networks. Some observed properties of OSNs are the following.

(1) Large scale and on-line.

OSNs are massive networks that have their order (number of users) and size (number of relationships) in the millions, even billions. There 


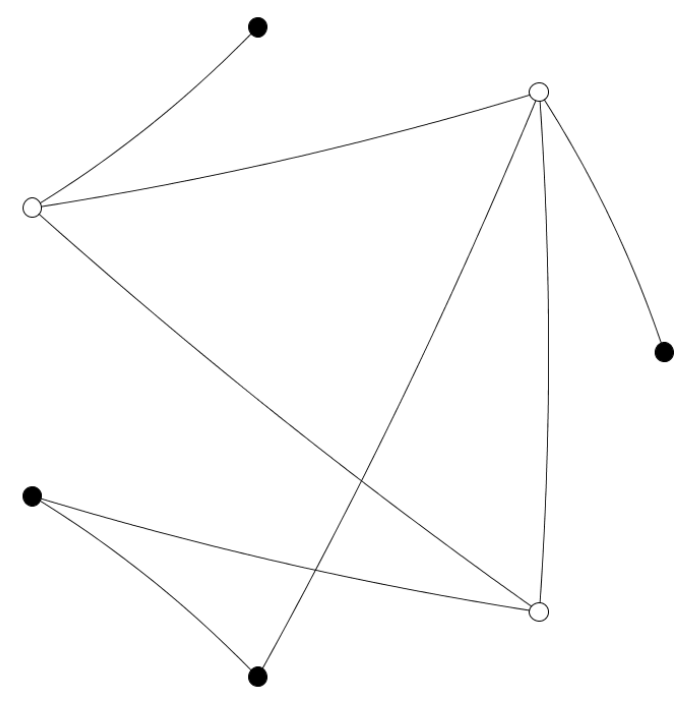

DS-DC Algorithm

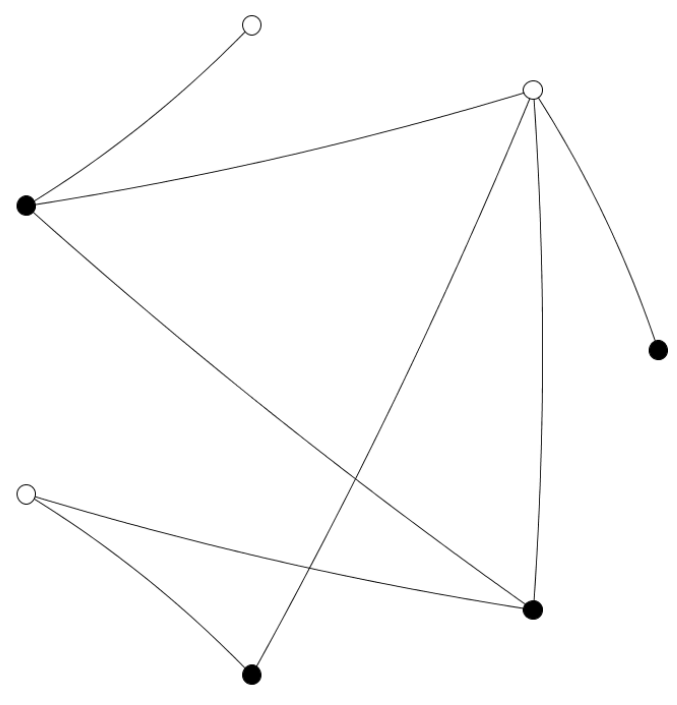

DS-RAI Algorithm

FiguRE 1.4. Dominating sets generated by the algorithms DS-DC and DS-RAI.

also exist some users with abnormally high degrees. The order of these networks is also dynamic: users are being removed and added all the time (this is known as the on-line property).

(2) Small world property and shrinking distance.

A famous chain letter experiment carried out by Stanley Milgram in 1967 [34] examined the average path length for the social networks of people in the United States. The experiment consisted of giving several letters to random people in different states addressed to a Boston stockbroker. The person would then be asked to send the letter onto other people whom they think who might know the stockbroker. Although only $20 \%$ of the letters were able to reached the stockbroker, on average, they took 6 steps through the social network. This was the beginning of the concept of the small world structure within networks. 
The result of this experiment is known as the six degrees of separation. It is the theory that it is possible for a connection to be made between highly disparate social populations in a small number of steps. After many years, the small-world property is well-studied, and appears in the literature of many disciplines such as sociology, biology, physics, and information technology; see Chapter 2 of [8].

The study of small-world property within complex networks was first introduced by Watts and Strogatz in 1998 [44]. Networks with this property were noted to have a low diameter of $O(\log n)$ (or average distance of $O(\log \log n))$ and a high clustering coefficient (higher than a random binomial distributed graph with the same number of nodes and average degree). A more mathematical definition of clustering coefficient and random graphs will be presented in the next section. In the papers $[\mathbf{1}, \mathbf{2}, \mathbf{2 3}, \mathbf{2 6}, \mathbf{3 5}]$, the small-world property have been observed (in Cyworld, Flickr, LiveJournal, MySpace, Orkut, Twitter and YouTube). Twitter has been observed to have a high clustering coefficient and a low diameter of 6 in $[\mathbf{2 3}, \mathbf{2 6}]$. In addition, it has been found in Cyworld [2], Flickr and Yahoo!360 [25] that their diameter shrinks over time.

(3) Power-law degree distributions.

Power laws were first studied in 1896 in Pareto's work [38] on income distribution. The power law degree distributions represents an undemocratic nature. There will be many people with low number of 
friends and a few people with a significantly higher number of friends. It has been reported that the power law in- and out-degree distribution exist for Flickr, LiveJournal, Orkut, YouTube [35] and Twitter [23]. Golder et al. [20] analyzed 4.2 million users from Facebook and found that it follows a power law. In addition, the degree distributions amongst the social networks studied in [35] were found to be similar. A definition of power law degree distributions will be given in Subsection 1.3.1.

(4) Densification power law.

This occurs when the average degree of the network grows to infinity as the order of the network increases. A graph $G$ follows a densification power law if there exist a constant $a \in(1,2)$ such that $|E(G)|$ is proportional to $|V(G)|^{a}$. In $[\mathbf{2}]$, it was documented that Cyworld (a South Korean social network) exhibit this densification power law.

(5) Bad spectral expansion.

OSNs are often divided into clusters. A common trait about them is that they contain communities, which is a characteristic of social organization $[\mathbf{3 6}]$. In these tightly knit clusters, the number of links within them are significantly higher than the links between other groups or clusters. Because of this structure, these networks have bad spectral expansion properties due to small gaps between the first and second eigenvalues of their adjacency matrices [18]. 
(6) Component structure.

In [25], Kumar et al. classified users of Flickr and Yahoo!360 into three categories: singletons (isolated nodes), the giant component (dense core of low (shrinking) diameter) and the middle region (isolated communities with star-like structure).

Two main properties that are particularly interesting are the power-law degree distributions and small world-property. A detailed description of each follows.

1.3.1. Power Law Degree Distribution. Let $G$ be a graph and $k$ a positive integer. The number of vertices of degree $k$ in $G$ is defined as:

$$
N_{k, G}=\left|\left\{x \in V(G): \operatorname{deg}_{G}(x)=k\right\}\right| .
$$

Since real-world complex networks are dynamic, for simplicity, we let $|V(G)|=t$ (this allows us to remember that the number of vertices within a system changes with time). A graph follows a power-law degree distribution if each degree $k, \frac{N_{k, G}}{t} \sim k^{-\beta}$, for a fixed real constant $\beta>1$. Here, $\beta$ is called the power law exponent. Since the function $f(k)=k^{-\beta}$ decays to 0 as $k$ tends to $\infty$ polynomially (not exponentially), the power law degree distribution is also known as a heavy-tailed distributions. See Figure 1.5 for a visualization of the general shape of a power law distribution. A graph for which the power law holds is known as a power law graph.

By taking the logarithms of the degree distribution, we will obtain an approximate straight line with slope $-\beta$. The power law usually exists for a 


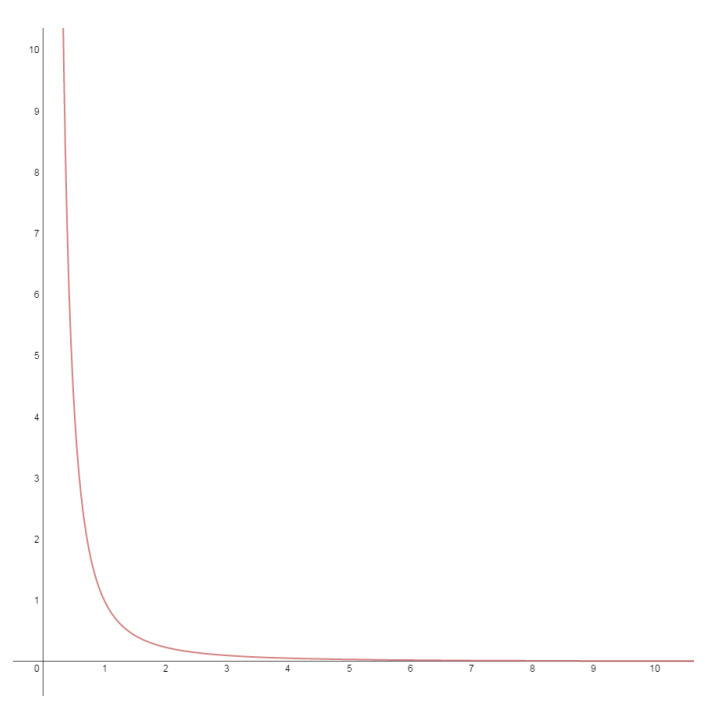

FiguRE 1.5. General shape of a power-law distribution.

specific interval of degrees with some deviation for nodes with small or large degrees. See Figure 1.6 and 1.7 for real-world examples of log-log plots.

$$
\log \left(N_{k, G}\right) \sim \log (t)-\beta \log (k)
$$

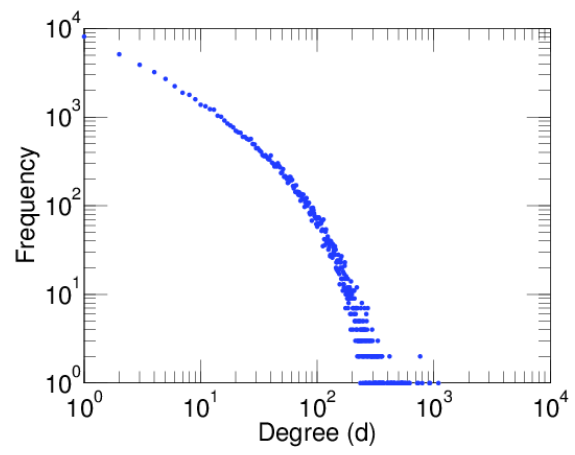

Figure 1.6. A log-log plot for Facebook friendships [43].

1.3.2. Small World Property. Small world graphs were first introduced in $[\mathbf{4 4}]$ where Watts and Strogatz defined the average distance (characteristic path length) that measured global distances in a graph and the measure for "cliquishness" of neighbourhoods. Small world graphs with order $t$ satisfy the following bound: $\operatorname{diam}(G)=\Theta(\log (t))$. However, when there 

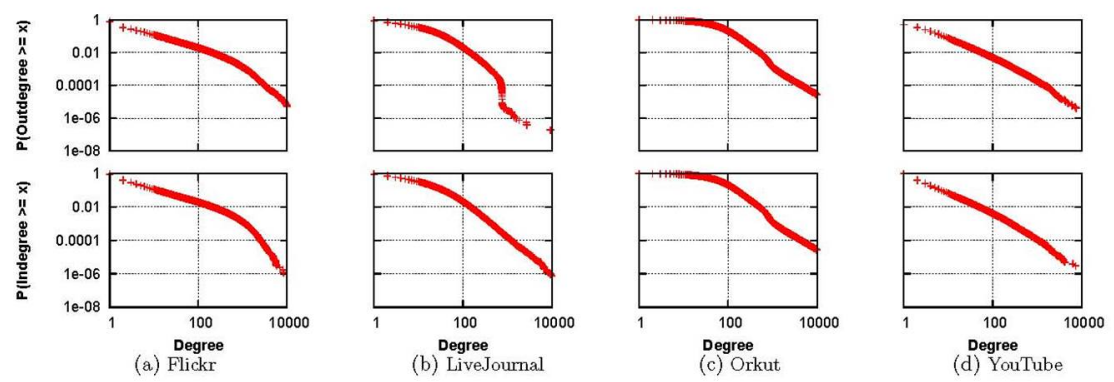

Figure 1.7. Log-log plots for Flickr, LiveJournal, Orkut and Youtube demonstrating the existence of power law degree distribution [35].

is an isolated vertex in a graph, this causes its diameter to be infinite. Thus, another measurement for global distance is necessary.

The average distance for a connected graph with order $n, G$, is defined as:

$$
\begin{aligned}
L(G) & =\frac{\sum_{u, v \in V(G)} d(u, v)}{\left(\begin{array}{l}
n \\
2
\end{array}\right)} \\
& =\frac{2}{n^{2}-n} \sum_{u, v \in V(G)} d(u, v) .
\end{aligned}
$$

Watts and Strogatz [44] reported the small world property requires $L(G)$ to be much smaller than the graph's order. It is demanded that

$$
L(G)=\Theta(\log \log n)
$$

A measurement that measures local density is the clustering coefficient. This coefficient should be larger relative to a random graph with the same order and size. Given a graph $G$ with order $n$ and $x \in V(G)$, define: 


$$
\begin{aligned}
C(x) & =\frac{|E(N(x))|}{\left(\begin{array}{c}
\operatorname{deg}(x) \\
2
\end{array}\right)} \\
& =\frac{2|E(N(x))|}{\operatorname{deg}(x)(\operatorname{deg}(x)-1)} .
\end{aligned}
$$

The clustering coefficient of a graph $G, C(G)$, is the average of all the clustering coefficients over all the vertices, which equates to:

$$
\begin{aligned}
C(G) & =\frac{1}{n} \sum_{x \in V(G)} C(x) \\
& =\frac{2}{n} \sum_{x \in V(G)} \frac{|E(N(x))|}{\operatorname{deg}(x)(\operatorname{deg}(x)-1)} .
\end{aligned}
$$

The value of the clustering coefficient is a rational number within $[0,1]$. Complete graphs (that is, ones that contain all possible edges) will have a clustering coefficient of 1 , while graphs without triangles have a clustering coefficient of 0 .

1.3.3. Modelling On-line Social Networks. There have been attempts to design a mathematically rigorous model that can simulate observed properties of OSNs. A model is said to simulate a property when a graph generated by the model satisfies the property, with high probability.

We provide here a brief list of some of these models and their properties. The preferential attachment (PA) model $[5,7]$ (satisfying properties 1 to 3), the geo-protean (GEO-P) model [11] (satisfying properties 1 to 4), the iterated local transitivity (ILT) model [10] (satisfying properties 1, 2, 4, 
5 (but not 3 and 6)), a model based on affiliation graphs [29] (satisfying properties 1 to 4), component structure model [25] (satisfying only property 6), the Forest Fire model, and Kronecker multiplication models [27, 28] (satisfying properties 1 to 4 ).

\subsection{Probability}

A few concepts of probability are needed in the thesis such as expectation and concentration. A discrete probability space $\mathcal{S}$ contains the following triplet, $(S, \mathcal{F}, \mathbb{P})$. The sample space, denoted $S$, is a non-empty and finite countable set. Set $\mathcal{F}$ is a collection of all possible subsets of $S$, these are called events. Finally, the function $\mathbb{P}: \mathcal{F} \rightarrow \mathbb{R}$ is known as the probability measure. The probability measure has the following properties:

(1) For all events $A, \mathbb{P}(A) \in[0,1]$, and $\mathbb{P}(S)=1$.

(2) If $\mathcal{F}$ is countable $\left(A_{i}: i \in I\right)$ and the events are pairwise disjoint, then

$$
P\left(\bigcup_{i \in I} A_{i}\right)=\sum_{i \in I} \mathbb{P}\left(A_{i}\right) .
$$

Since $\mathcal{F}$ is considered to be the power set of $S$, it does not need to be specified. An example of a probability space is the uniform probability space

on $S$ with $\mathbb{P}(A)=\frac{|A|}{n}$. The set $S$ is defined as being a finite set with $n>0$ elements. An element is chosen uniformly at random (or u.a.r.) when all elements have the same probability of being chosen. Like in this case, every element has probability of $\frac{1}{n}$ to be chosen from $S$. 
A discrete random variable, denoted $X$, is a function $X: S \rightarrow \mathbb{R}$ whose inputs are a sample space $S$ on the probability space $\mathcal{S}$. Some information about binomial distribution is required for this thesis. The probability mass function (p.m.f.), $f: \mathbb{R} \rightarrow[0,1]$, of $X$ is defined by $f(x)=\mathbb{P}(X=x)$. Given a $x \in \mathbb{R}$, we compute

$$
\mathbb{P}(X=x)=\sum_{\substack{s \in S, X(s)=x}} \mathbb{P}(\{s\}) .
$$

The first moment of a random variable or its expectation, written $\mathbb{E}(X)$, is defined as

$$
\mathbb{E}(X)=\sum_{s \in S} X(s) \mathbb{P}(\{s\}) .
$$

If $S$ is finite, then $\mathbb{E}(X)$ will also be finite.

When taking the expectation of multiple random variables, one property that will provide significant help is stated in Theorem 1.2.

Theorem 1.2. [21] If the random variables $X, Y$, and $X_{i}$ where $1 \leq i \leq n$, are defined on a probability space, then the following holds.

Linearity of Expectation. If $c_{i}$ is any real number constant where $1 \leq i \leq n$, then

$$
\mathbb{E}\left(\sum_{i=1}^{n} c_{i} X_{i}\right)=\sum_{i=1}^{n} c_{i} \mathbb{E}\left(X_{i}\right) .
$$


A binomial random variable $X$ has parameters $n$ and $p$ and has the $p . m . f$. $\mathbb{P}(X=i)=\left(\begin{array}{c}n \\ i\end{array}\right) p^{i}(1-p)^{n-i}$ while its expectation is $n p$ and its variance $n p(1-p)$. This simply counts the number of successes in $n$ independent trails with a probability of $p$ success. We state the following concentration inequality.

TheOREM 1.3. [21, 22] (Chernoff bounds) If $X$ is a binomial random variable, the following holds.

$$
\begin{aligned}
& \mathbb{P}(X \geq \mathbb{E}(X)+t) \leq \exp (-\mathbb{E}(X)) \phi(t / \mathbb{E}(X)) \\
& \mathbb{P}(X \leq \mathbb{E}(X)-t) \leq \exp (-\mathbb{E}(X)) \phi(-t / \mathbb{E}(X))
\end{aligned}
$$

where $\phi(x)=(1+x) \log (1+x)-x$ for $x \geq-1$, and $\phi(x)=\infty$ for $x<-1$. If $\varepsilon \leq 3 / 2$, then

$$
\mathbb{P}(|X-\mathbb{E}(X)| \geq \varepsilon \mathbb{E}(X)) \leq 2 \exp \left(-\frac{1}{3} \varepsilon^{2} \mathbb{E}(X)\right)
$$

We say an event $A_{n}$ holds asymptotically almost surely (a.a.s.) if it holds with probability tending to 1 as $n$ tends to infinity.

1.4.1. Random Graphs. Let $n$ be a positive integer, and let $p$ be a real number in $[0,1]$. The space of random graphs of order $n$ with edge probability $p$ is denoted as $G(n, p)$. Random graphs were first studied by Paul Erdős and Alfred Rényi in 1959 [17]. They are graphs that have a binomial degree distribution which follows $\mathbb{P}(G)=p^{|E(G)|}(1-p)^{\left(\begin{array}{c}n \\ 2\end{array}\right)-|E(G)|}$. 
These are graphs with a fixed vertex set $V$ and varying number of edges (two vertices are adjacent independently with probability $p$ ). The number of edges follows a binomial distribution and has expectation $\left(\begin{array}{l}n \\ 2\end{array}\right) p$, as there are $\left(\begin{array}{l}n \\ 2\end{array}\right)$ many possible edges each occurring with probability $p$. Although $G(n, p)$ represents a probability space of graphs, we abuse language and refer to it as the random graph with order $n$ and edge probability p. See Figure 1.8 for an example of a randomly drawn graph.

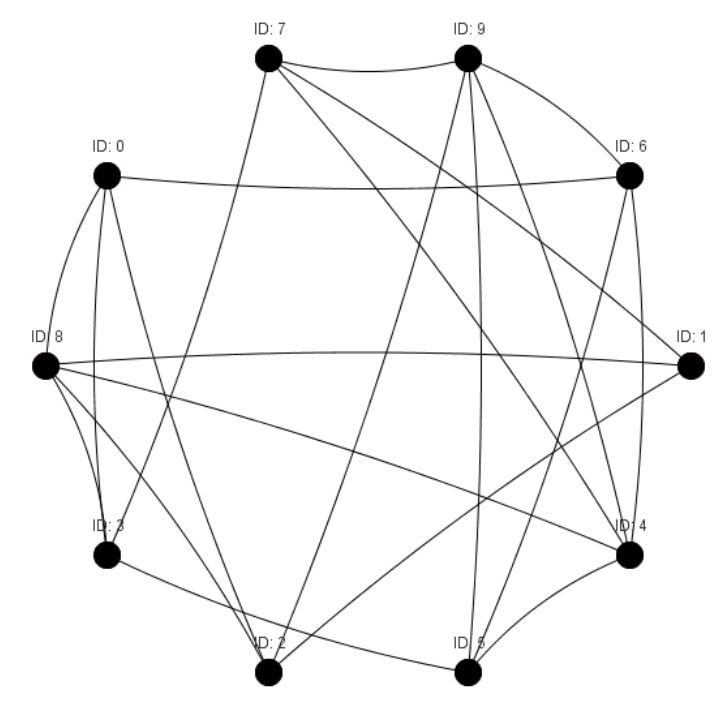

Figure 1.8. Sample graph from $G(n, p)$ where $n=10$ and $p=0.5$.

If we let the random variable $X$ to be the degree of any particular vertex, then it follows a binomial distribution. A binomial distribution histogram approximates a bell curve shape with a concentration around its expected value when $p$ is fixed and $n \rightarrow \infty$. The random variable $X$ is defined as the sum of random variables that follow a Bernoulli distribution (that is, it counts the number of vertices adjacent to the particular node): $X=\sum_{i=1}^{n} Y_{i}$. Note that a Bernoulli distribution is a simple distribution that allows a random 
variable to take the value of 1 with probability $q$ (in other terms, 0 with probability $q=1-p$ ). Thus, the expected value of a Bernoulli distribution is simply $p$ :

$$
\begin{aligned}
E(X) & =E\left(\sum_{i=1}^{n} Y_{i}\right) \\
& =\sum_{i=1}^{n} E\left(Y_{i}\right) \\
& =\sum_{i=1}^{n} p \\
& =n p,
\end{aligned}
$$

where the seconds equality follows by the Linearity of Expectation (Theorem 1.2). Concentration on the expected degree follows by use of the Chernoff bounds; see Theorem 1.3.

Figure 1.9 is the expected shape of a binomial distribution.

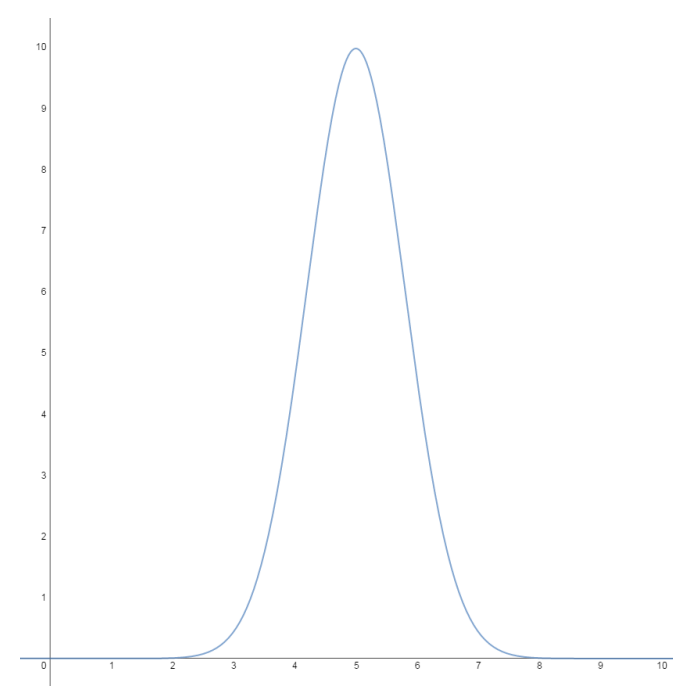

Figure 1.9. General shape of a binomial distribution. 


\subsection{Dominating On-line Social Networks}

One key property that is noticed in social networks is that people tend to have characteristics or attributes similar to their friends. The reason for this is because of social influence (adopting behaviours through word-ofmouth or imitation) and selection (forming relationships with people similar to you) [16]. The structure of these networks will vary due to these two forces. However, in the end, this causes people to have relationships with similar people. Social influence produces a network-wide uniformity while selection drives the network to smaller clusters [16]. An example of social influence was presented by Kramer et al. in 2014 [24] where they provided evidence of emotional contagion by manipulating the news feed for nearly 700,000 Facebook users.

With over a billion users and 70 billion friendships, Facebook is the world's largest on-line social network. In 2012, it was computed that Facebook had an average of 4 degrees of separation amongst their users [4]. This brings up an important popular subject of on-line social networks: information diffusion or the spread of (social) influence. Researchers worldwide have been drawn to try and model these processes along with their dynamic relationships. The spread of influence can take in many forms: a computer virus, a marketing ad, a disease, or even social influences (such as emotional states $[24])$. A good indicator of how susceptible a social network is to the spread of influence is the number of users required such that for every user not in this set is adjacent to at least one user in the set (that is, a dominating set). 
In Section 1.2.1, we defined the minimum cardinality of the domination set as the domination number of the network. These are the agents that potentially have the most influence in the network. By sending a message, a virus, an emotion, through these users, that information gets rapidly spread out to the entire network. Other various of applications that uses this domination concept are: efficient data routing [40], detecting highly significant optimized subsets of proteins in protein-protein interaction networks (PPIs) [33], and developing a dynamic backbone for Mobile Ad-Hoc Networks (MANETs) for routing and broadcasting [45]. Domination also plays a role in distributed computing and network controllability [15].

In addition, other concepts have been studied to determine how sensitive a graph is to influence like social elites [14]. Using the domination number to analyze a network only allows us to potentially capture such agents in the network. A more sophisticated measurement would be required to analyze graph sensitivity to influence. The paper published by Kramer et al. [24] in 2014 provided empirical evidence of emotional contagion by manipulating the Facebook news feed of over 600,000 Facebook users. The researchers did not change the content of what the users were posting but only restricted what they saw based on keyword analysis. They found that users who were subject to more positive posts tend to post more positive content. Similarly the users subject to negative content were more likely to post something negative. This brings up an important fact that we as humans do not required human-human interactions in order to be influenced. A good way to see the significance of 
a dominating set in this example is to imagine such a set existed with a size of say, 1,000. That means if these 1,000 users were to post positive content, then the entire networks (potentially consisting of millions of users) would be influenced.

\subsection{Outline of Thesis}

In Chapter 2, the MGEO-P model will be introduced with a brief background of its development from the GEO-P model, and how it relates to the concept of Blau space and its properties. Some simulations are presented. Within Chapter 3, upper bound domination numbers are computed for the Facebook 100 (also known as FB100) graphs and corresponding MGEO-P samples. In Chapter 4, a new variation of the MGEO-P model is introduced and analyzed: the Distance MGEO-P model. And finally in Chapter 5, we summarize our findings and present open problems and directions for future work. 



\section{CHAPTER 2}

\section{Memoryless Geometric Protean Model}

\subsection{Background}

The memoryless geometric protean model (MGEO-P) was first introduced by Bonato et al. in 2014 [9]. This is a variation on the geometric protean model (GEO-P) that allows us to approximate the GEO-P model without costly sampling. The MGEO-P model has its edges arrive based on a given distance metric in $m$-dimensional Euclidean space $\mathbb{R}^{m}$. This space closely mirrors Blau space [13], a construction in the social sciences. It is a multidimensional coordinate system where the dimensions are defined by social demographic variables. The relative position of the vertices within Blau space are guided by the principle of homophily [14]. That is, vertices that are closer to each other tend to have similar socio-demographic characteristics. Examples of Blau space dimension variables are age, sex, location, education and income. The sociologist Peter Blau introduced this notion which bears his name. He was the first to take individuals in a social network and abstract them to nodes in a multi-dimensional space. He had the idea that "social forces" are the overlaps of social demographic characteristics and that population structure can influence human behaviour [6]. It was later on more fully developed by Miller McPherson $[\mathbf{3 0}, \mathbf{3 1}, \mathbf{3 2}]$. 


\subsection{The Model}

The MGEO-P model consists of five parameters $\operatorname{MGEO-P}(n, m, \alpha, \beta, p)$ : the number of nodes $n$, the dimension $m$, the attachment strength parameter $0<\alpha<1$, the density parameter $0<\beta<1-\alpha$ and the probability of connection $0<p \leq 1$. Here, the parameters $n$ and $m$ are positive integers that are greater than 0 and $\alpha, \beta, p$ are real numbers. The network starts out empty. During each iteration of the process, a new vertex is added to the network. There will be a total of $n$ steps resulting in a graph of order $n$. For each of the $n$ steps, a vertex $v$ is created and given a random rank $r_{v}$ and random position $q_{v} \in \mathbb{R}^{m}$ within the unit-hypercube $[0,1]^{m}$. The random rank is chosen from the unused remaining ranks from 1 to $n$. A unit-hypercube side has length one unit with $2^{m}$ vertices in $\mathbb{R}^{m}$ with coordinates $\{0,1\}^{m}$. The volume of space that a node influences is $r_{v}^{-\alpha} n^{-\beta}$. The radius of a node can be obtained by taking the $m$ th-root of the volume of the node and dividing it by two. Thus, each vertex has a radius of influence given by:

$$
I\left(r_{v}\right)=\frac{1}{2}\left(r_{v}^{-\alpha} n^{-\beta}\right)^{1 / m}
$$

An undirected edge is created between $v$ and any preexisting vertex $u$ with probability $p$, if $D(v, u) \leq I\left(r_{u}\right)$. The distance is computed using the infinitynorm. The infinity-norm is defined as $\|x\|_{\infty}=\max \left\{\left|x_{1}\right|,\left|x_{2}\right|, \ldots,\left|x_{m}\right|\right\}$. The distance used in the model is computed as follows:

$$
D(u, v)=\min \left\{\left\|q_{v}-q_{u}-z\right\|_{\infty}: z \in\{-1,0,1\}^{m}\right\}
$$


The $z$ term within the distance formula allows the geometric space to be symmetric and "wrap" around like a torus. This process is repeated until all the vertices have been placed within the unit-hypercube.

\subsection{Simulations}

Figure 2.1 demonstrates the process of simulating MGEO-P $(3,2,0.17,0.27,1)$ which we explain here. For simplicity, we assume that the probability of connection $p$ for the MGEO-P model is 1 . In reality, we know this is not the case. A more realistic value would be less than 0.5 . The network initially starts out empty. In the first step, a node is placed with the coordinates $(0.1894,0.1284)$ and a radius of 0.431081 . There are no pre-existing nodes to check for any edges. During the second step, another node is generated at $(0.7439,0.7232)$ and a computed radius of 0.406416 . The procedure continues with checking if the distance between the current generated node and any pre-existing node is less than the pre-existing node's radius. In this case, $D(u, v)=D(1,2)=0.445500 \not \leq 0.431081$. Therefore, an edge is not placed between these two nodes. Finally, the third node is placed in the space at $(0.4200,0.9213)$ with radius 0.392648 . Repeating the process, computing the distance of nodes one and three $D(1,3)=0.2306 \leq 0.431081$ tells us an edge is placed. Likewise, for nodes two and three $D(2,3)=0.3239 \leq 0.406416$, thus, an edge is placed.

Figure 2.2 shows visual representations of graphs generated by the MGEO-

P model using JUNG 2.0.1 (Java Universal Network/Graph) framework which was released in early 2010. In the original GEO-P model, the process does 


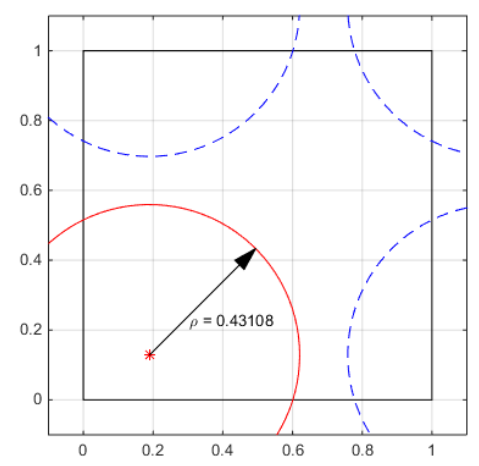

(A)

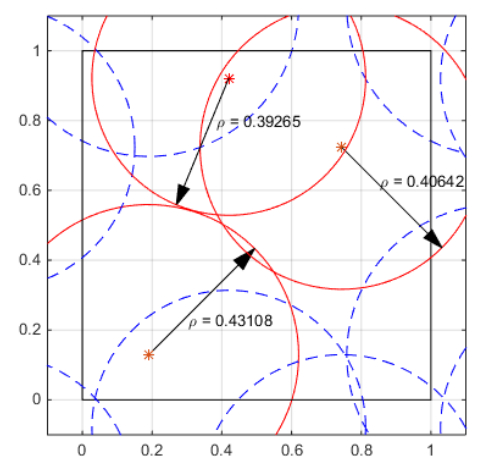

(c)

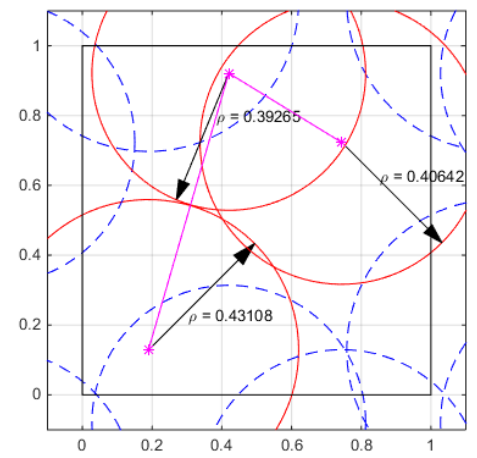

(E)

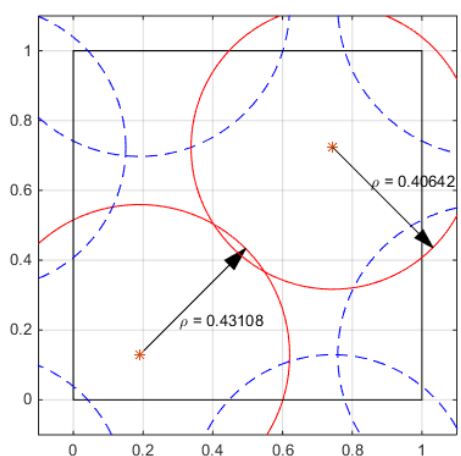

(в)

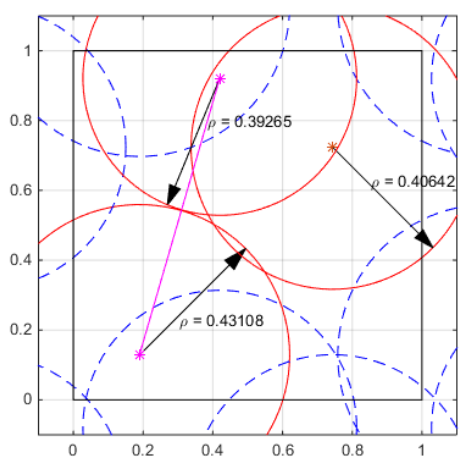

(D)

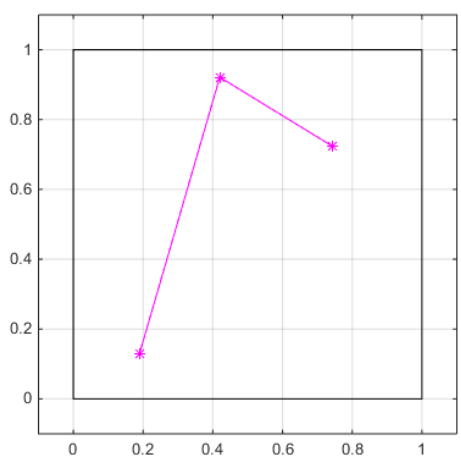

$(\mathrm{F})$

FIGURE 2.1. Time steps of a MGEO-P $(3,2,0.17,0.27)$ sample. The unit square is given by the black interior rectangle from 0 to 1 . The broken blue circles are copies of nodes illustrating the torus metric. (A) Time step 1: First node is added to the network. (B) Time step 2: Second node is added to the network. No nodes are overlapping, thus no edges. (C) Time step 3: Third node is added to the network. Overlaps with node one and two. (D) An edge is placed in between nodes one and three. (E) An edge is placed in between nodes two and three. $(F)$ Final graph without node radii. 
not end there. The procedure is repeated until a random stop time, where in each step the least-recently added vertex will be removed and a new one inserted.
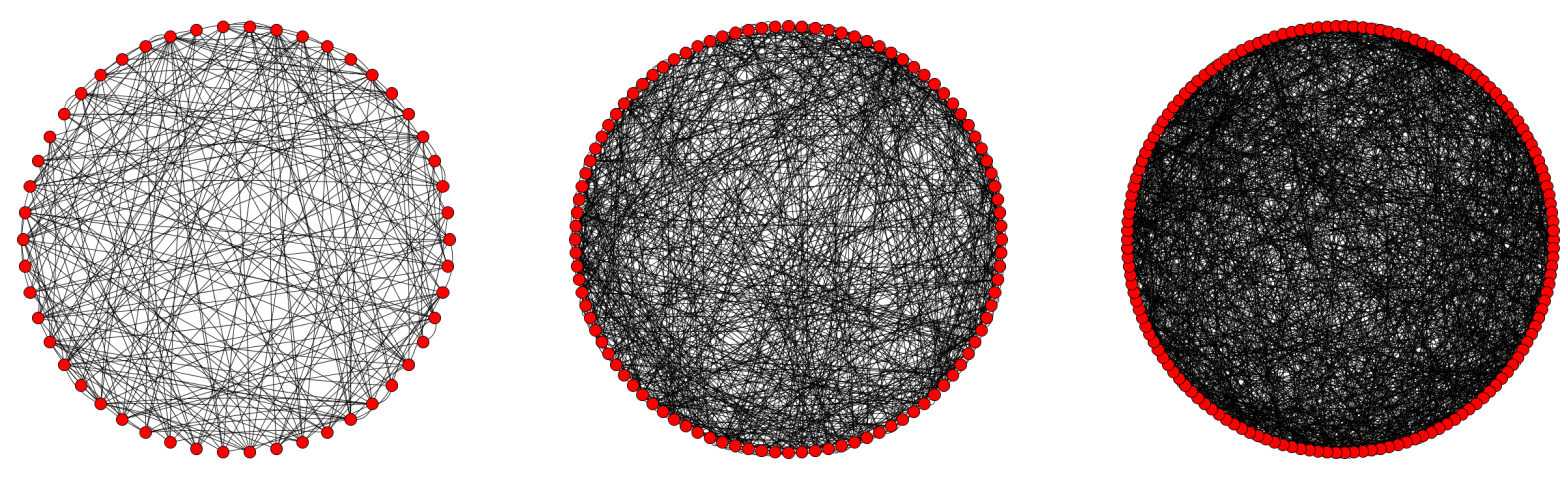

MGEO-

MGEO-

$\mathrm{P}(50,3,0.17,0.27,1)$

$\mathrm{P}(100,3,0.17,0.27,1)$

\section{MGEO-}

$\mathrm{P}(150,3,0.17,0.27,1)$
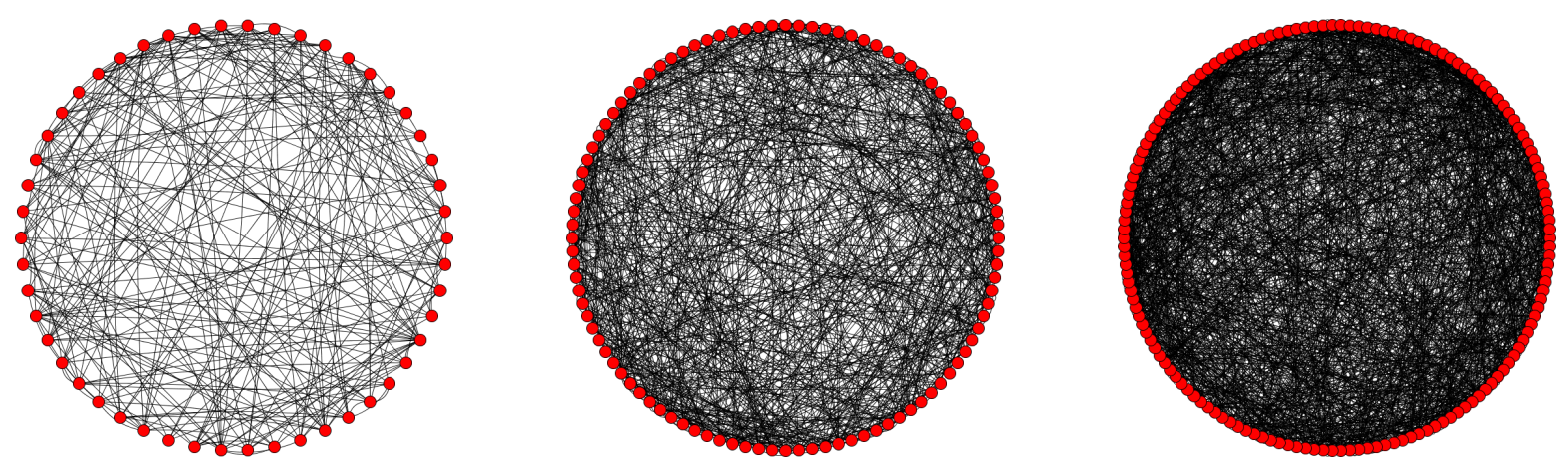

MGEO-

$\mathrm{P}(50,4,0.17,0.27,1)$

MGEO-

$\mathrm{P}(100,4,0.17,0.27,1)$
MGEO-

$\mathrm{P}(150,4,0.17,0.27,1)$

Figure 2.2. Samples of the MGEO-P $(n, m, \alpha, \beta, p)$ model, with various choices of parameters.

As mentioned in Chapter 3 (Domination Number in Facebook 100 and the MGEO-P Model), the MGEO-P parameters were computed for Facebook 100 data [12]. Tables A.1, A.2, A.3 show a preliminary analysis of the MGEO-P samples using the Facebook 100 parameters. The tables provide graph properties such as number of nodes, number of edges, average degree, maximum 
degree and density. Density is simply a measurement of how many edges there are relative to the maximum number of edges. It is defined as:

$$
D=\frac{2 E}{V(V-1)}
$$

Therefore, complete graphs have a density of 1 and graphs with no edges a density of 0 . A complete graph for the MGEO-P model would have a attachment strength parameter and density parameter of 0 to keep each nodes radius of influence uniform. These results will be used to make comparisons between the MGEO-P model, the modified MGEO-P model, and the Facebook data to draw any conclusions.

The following theorems about the MGEO-P model appear in [9] along with their proofs. We omit the proofs here as they are beyond the scope of this thesis. A useful result that follows from Theorem 2.1(1) is that a.a.s. the minimum degree $\delta$ is at least $(1+o(1)) p n^{1-\alpha-\beta}$.

TheOREM 2.1. [9] Asymptotically almost surely, the following properties hold for graphs generated by MGEO-P.

(1) If $v$ is a node of a $M G E O-P(n, m, \alpha, \beta, p)$ sample with rank $R$ and age $i$, then

$$
\operatorname{deg}(v)=\left(\frac{i-1}{n-1} \frac{p}{1-\alpha} n^{1-\alpha-\beta}+(n-i) p R^{-\alpha} n^{-\beta}\right)\left(1+\mathcal{O}\left(\sqrt{\frac{\log ^{2}(n)}{n^{1-\alpha-\beta}}}\right)\right)
$$


(2) The average degree of a vertex of $M G E O-P(n, m, \alpha, \beta, p)$ is

$$
d=\frac{p}{1-\alpha} n^{1-\alpha-\beta}\left(1+\mathcal{O}\left(\sqrt{\frac{\log ^{2}(n)}{n^{1-\alpha-\beta}}}\right)\right) .
$$

(3) The diameter of MGEO-P $(n, m, \alpha, \beta, p)$ is $n^{\Theta\left(\frac{1}{m}\right)}$.

(4) If $k$ is chosen such that

$$
n^{1-\alpha-\beta} \log ^{\frac{1}{2}}(n) \leq k \leq n^{1-\frac{\alpha}{2}-\beta} \log ^{-2 \alpha-1}(n),
$$

then $M G E O-P(n, m, \alpha, \beta, p)$ satisfies

$$
N_{\geq k}=\left(1+\mathcal{O}\left(\log ^{-\frac{1}{3}}(n)\right)\right) \frac{\alpha}{1+\alpha} p^{\frac{1}{\alpha}} n^{\frac{(1-\beta)}{\alpha}} k^{-\frac{1}{\alpha}},
$$

where the number of vertices of degree at least $k$ is denoted by $N_{\geq k}$.

These theorems demonstrate that the model a.a.s. exhibits a power-law degree distribution. A few samples of the model were simulated with their histogram and log-log plot computed to provide evidence that a power-law distribution appears in graphs generated by the model. See Figure 2.3 and Figure 2.4. As expected, the MGEO-P model follows a power-law degree distribution and has a linear slope in its log-log plot, while $\mathrm{G}(n, p)$ follows more of a binomial degree distribution with a noisy log-log plot. 

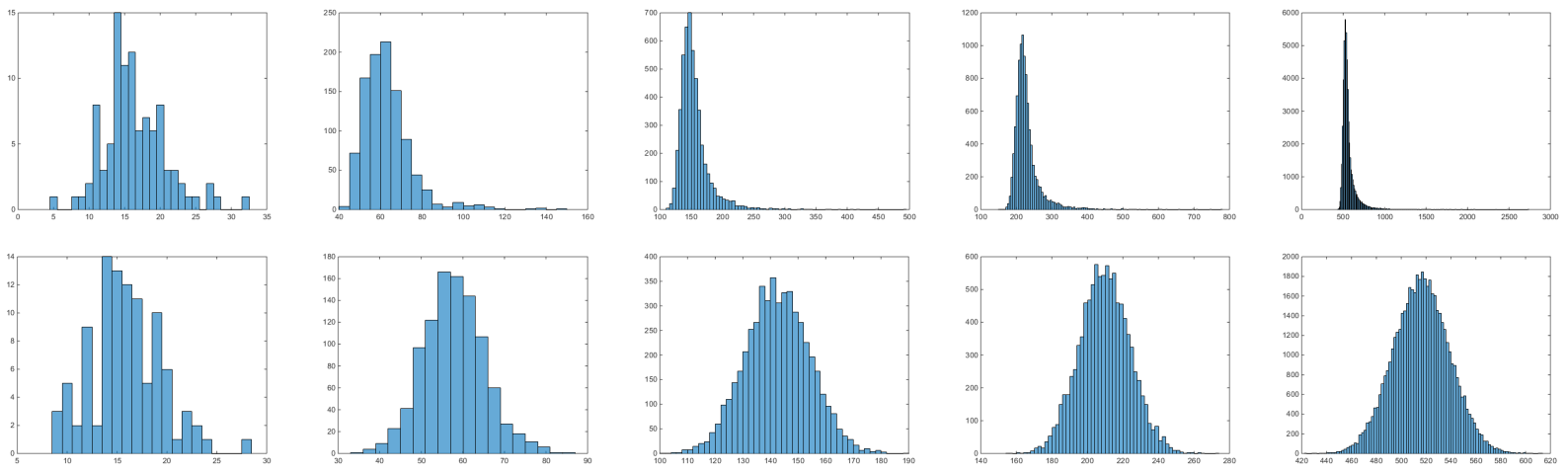

Figure 2.3. Degree distribution of $\operatorname{MGEO-P}(n, 4,0.17,0.27,1)$ samples and $\mathrm{G}(n, p)$ model for $n=$ $100,1000,5000,10,000$ and 50,000. Note that $p$ was chosen in $\mathrm{G}(n, p)$ so that the graphs sampled have the approximate same average degree as the corresponding MGEO-P samples. The size of each bin were automatically computed using MATLAB's automatic binning algorithm.
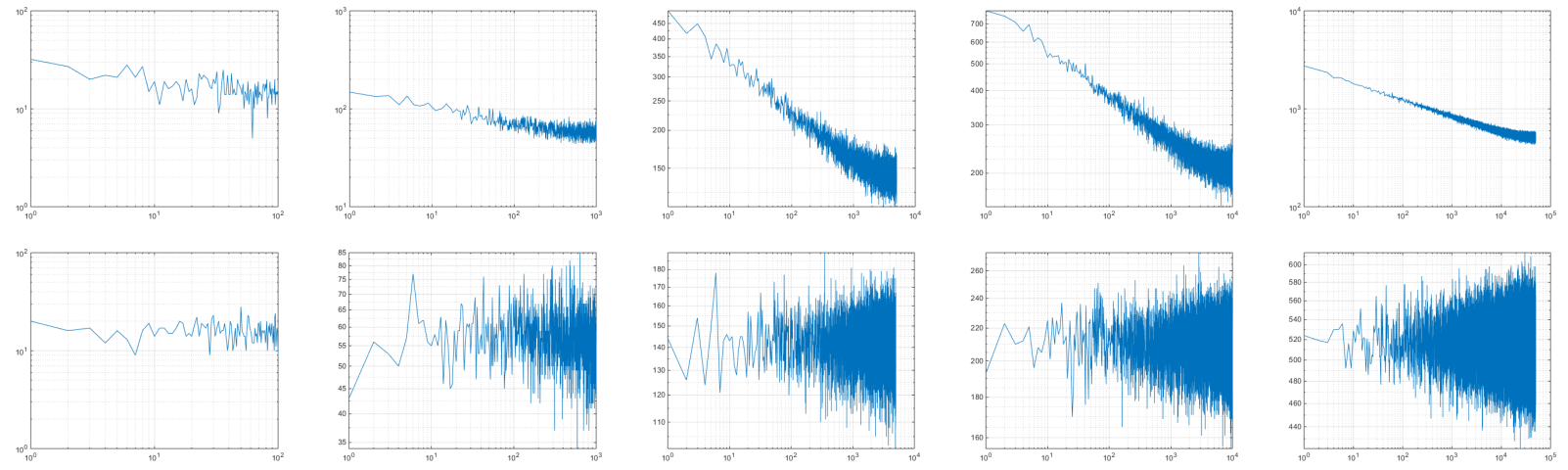

Figure 2.4. Log-log plots of $\operatorname{MGEO}-\mathrm{P}(n, 4,0.17,0.27,1)$ samples and $\mathrm{G}(n, p)$ model for $n=$ $100,1000,5000,10,000$ and 50,000. Note that $p$ was chosen in $\mathrm{G}(n, p)$ so that the graphs sampled have the approximate same average degree as the corresponding MGEO-P samples. 
CHAPTER 3

\section{Domination Number in Facebook 100 and the MGEO-P Model}

\subsection{Facebook 100}

To investigate the domination number and its values on social networks, we need a data set that resembles a natural on-line social network. We randomly generate MGEO-P samples since they follow most OSN properties. However in [9], the parameters of a Facebook dataset were computed for the MGEO-P model. Using these parameters, model samples can be simulated and tested. The social network data used are known as the Facebook 100 (or FB100) graphs. They were distributed and anonymized by Mason Porter who worked at Facebook. It contains 100 Facebook network samples from 100 universities from the United States in September 2005 [41] as separate networks. Their order and size vary from 700 users to 42,000 users and 16,000 friendships to 1,590,655 friendships. For this thesis, the data that was used were the friendship networks of each institution as separate graphs. These graphs provide a single snapshot of subgraphs of the Facebook network.

The Facebook 100 data represents an historical snapshot of agents joining the network over time that can describe different stages of institutions within the network. For example, some users might have joined the network earlier or later. The original released data that has been analyzed in [41] contains 
additional information such as gender, class year, major, high-school and residence.

Before investigating the domination sets within the FB100, some preliminary analysis should be done to indicate any significant properties about these social networks. It should be noted that the minimum degree for each dataset in the Facebook 100 network is 1 and is excluded from the tables. Tables A.16, A.17, A.18 provides the preliminary analysis of FB100.

\subsection{Domination Number within Facebook 100}

Given the FB100 graphs, the dominating set algorithms DS-RAI and DS-DC (as described in Chapter 1) were executed multiple times to provide an idea of what the upper bounds of the domination number of on-line social networks could be. Tables A.19, A.20, A.21, A.22 and A.23 in the Appendix, show the results of the DS-RAI algorithm for the $k$-cores, where $1 \leq k \leq 5$. The 0-core (that is, the original graph) was not accounted for because the Facebook 100 graphs do not have any isolated vertices thus, making the 0-core graphs equivalent to the 1-core graphs.

The DS-RAI algorithm performs the fastest and provides a good indication of what the upper bounds are for the Facebook 100 graphs. The DS-DC algorithm improves on these upper bounds at the cost of a longer execution time. The following Tables A.24, A.25, A.26, A.27 and A.28 in the Appendix, provides the dominating set size achieved by the DS-DC algorithm for the $k$-cores, where $1 \leq k \leq 5$. The DS-DC algorithm provides upper bounds 


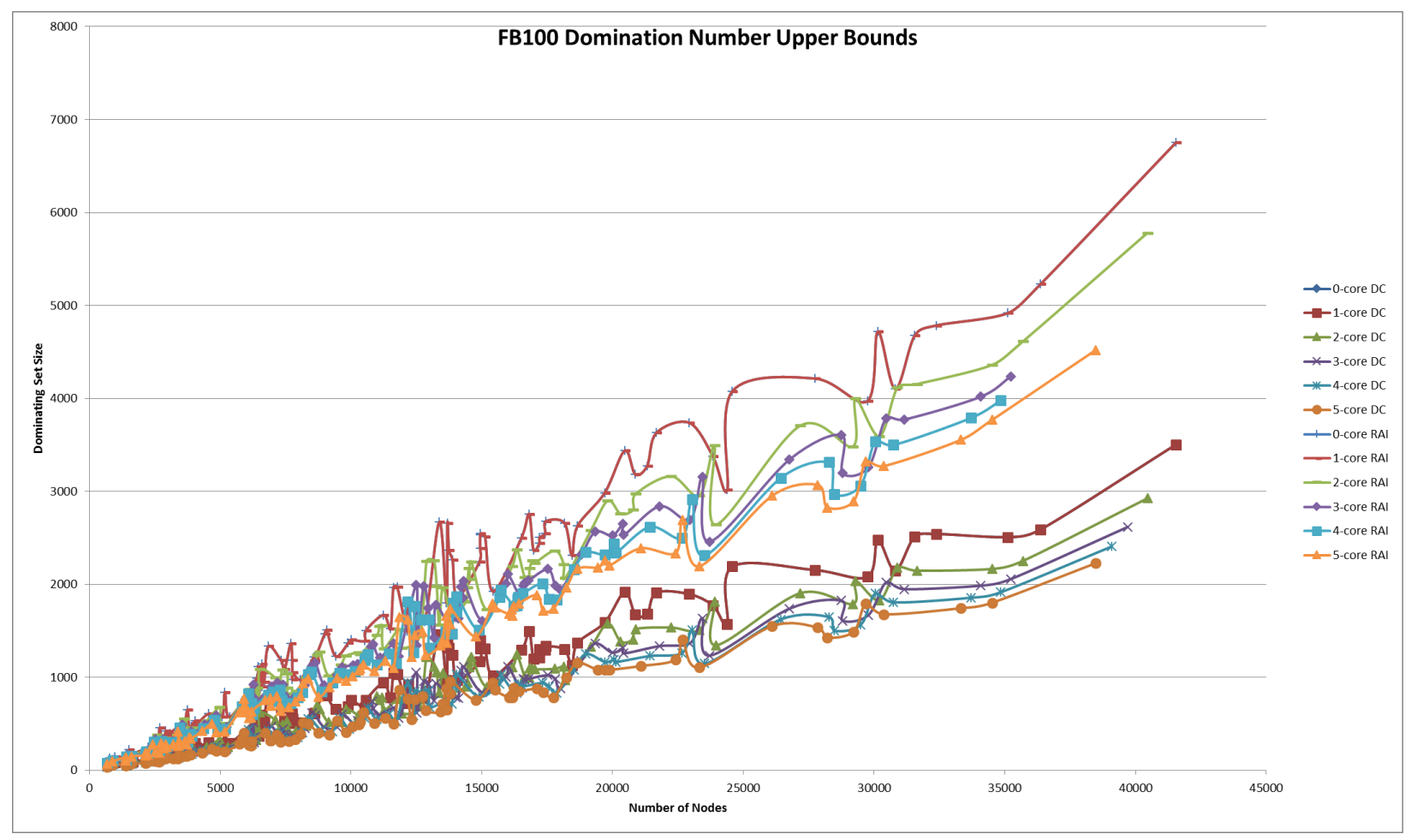

Figure 3.1. Domination number upper bounds of the Facebook 100 graphs.

that are approximately $75 \%$ of the order of the dominating sets obtained via DS-RAI.

The data represented in the plot Figure 3.1 are the dominating set sizes for the Facebook 100 graphs with DS-DC and DS-RAI relative to the graph order. Since our graphs are quite small when considering asymptotic bounds, the data progression can be consider either linear or sub-linear. The general upper bound mentioned in Theorem 1.1 suggests that the upper bound is potentially linear. Comparing the theoretical and computed bounds shows that the upper bound mentioned by Alon and Spencer in $[\mathbf{3}]$ is quite significantly higher than the computed bounds for the FB100 graphs. The comparison plot is shown in Figure 3.2, where the minimum degree $\delta$ used was considered to be 5 since we took up to the 5 -core. 


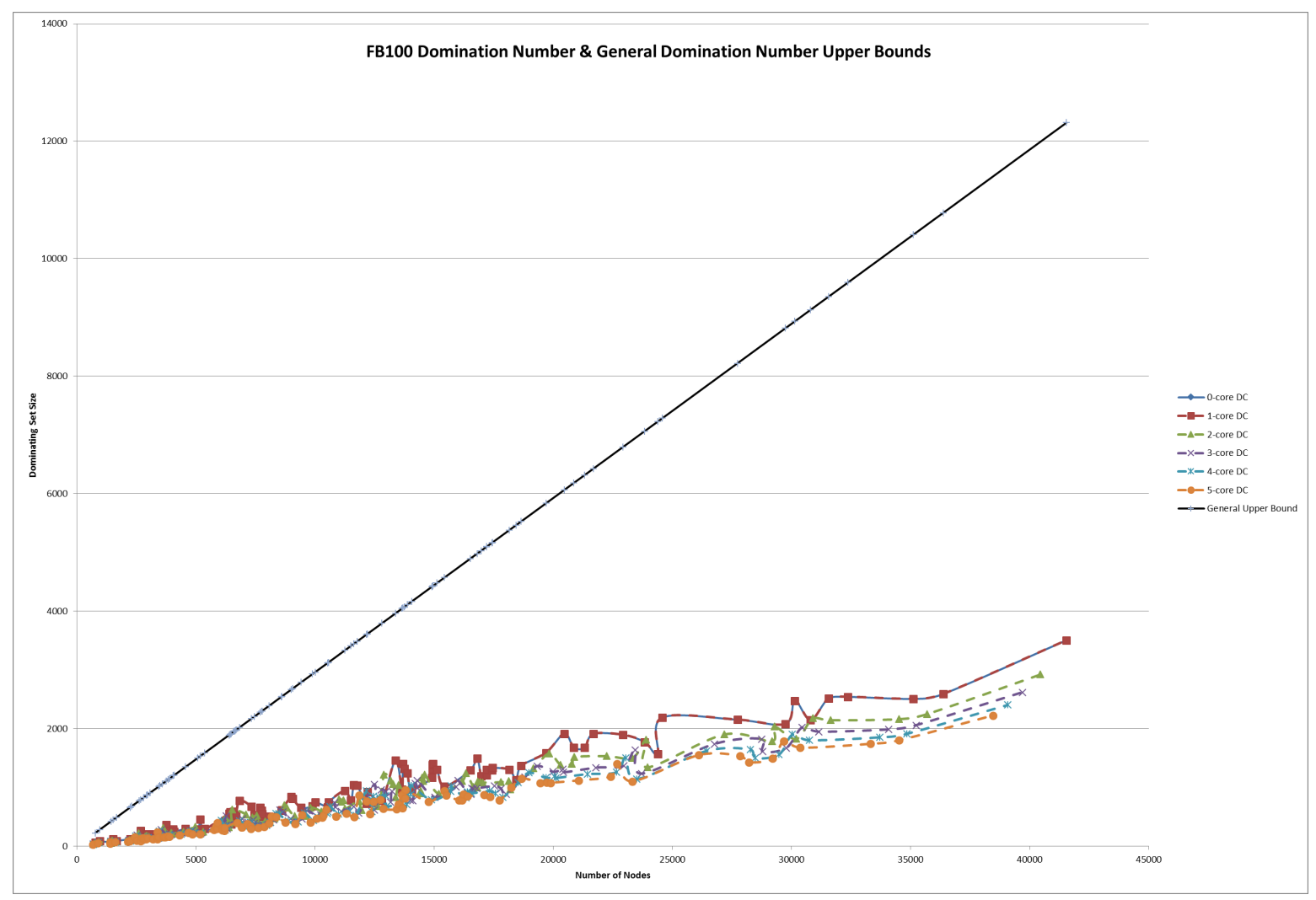

FiguRE 3.2. Domination number upper bounds of the Facebook 100 graphs with Theorem 1.1 upper bound $(\delta=5)$, presenting a significant overestimation.

\subsection{Domination Number within MGEO-P}

MGEO-P samples generated from the Facebook 100 parameters in [9] were used to provide an upper bound of the domination number for the model. For simplicity, when the samples were generated the probability parameter $p$ was considered to be 1 . To obtain an appropriate MGEO-P sample for the FB100, percolation must be done. This is the process of randomly removing edges until the graph has a set number of edges. For graphs with less edges than those in the data set, they are left intact. Just like in [9], our samples were percolated to match the number of edges of the Facebook data. Intuitively, one would expected that the graphs generated using the process of 


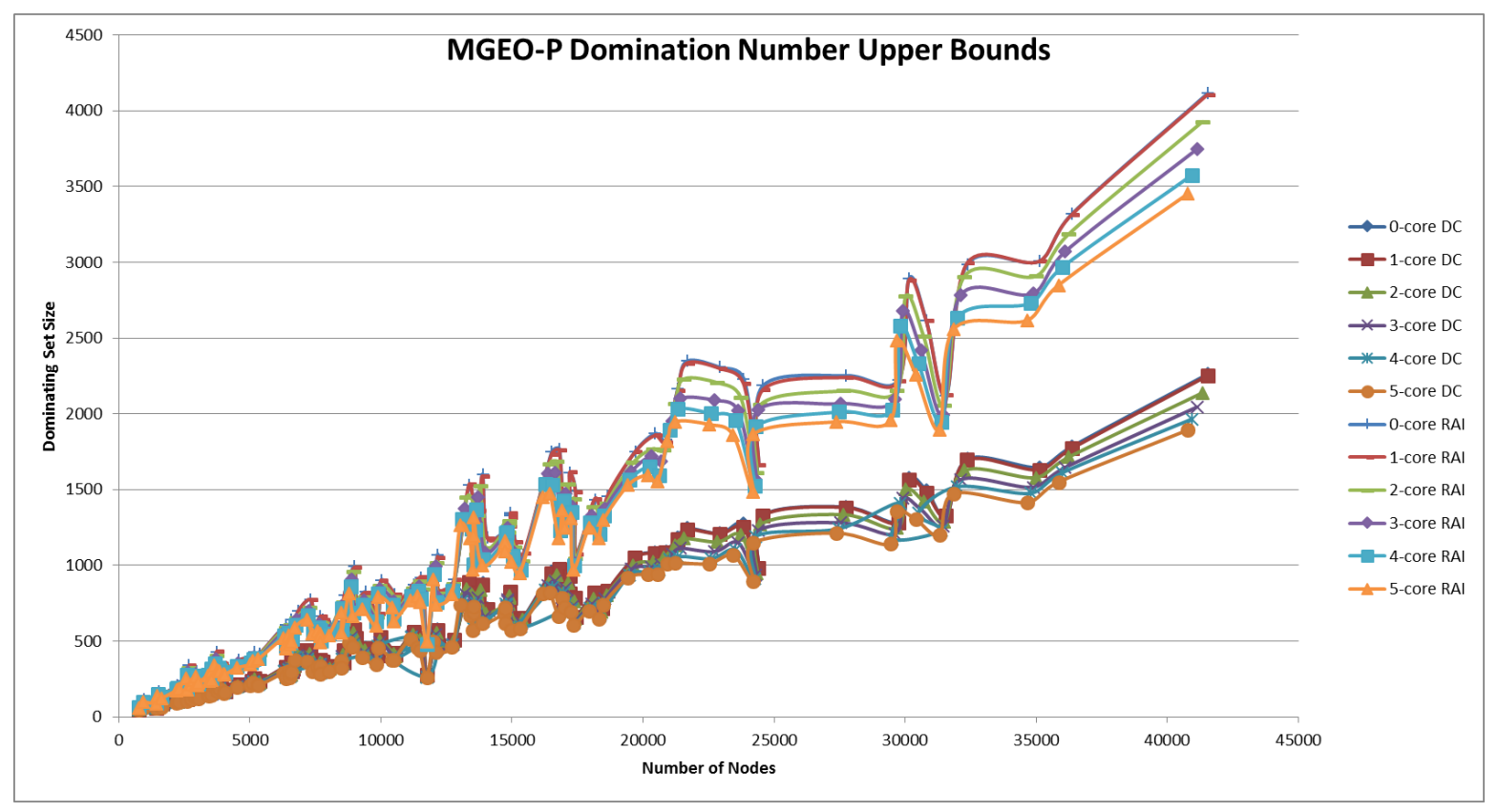

FigurE 3.3. Domination number upper bounds of MGEO-P samples corresponding to the Facebook 100 graphs.

percolating to be quite similar than the original data. This is indeed true and is seen when comparing the preliminary analysis of Table A.1, A.2, A.3 and Table A.16, A.17 and A.18. A noticeable difference is the maximum degree of the samples. One should expect a larger dominating set for the MGEO-P samples because their maximum degrees are lower than the original data. The upper bound of the domination number is shown in Figure 3.3.

A recent finding in $[\mathbf{1 2}]$, provides a theorem regarding the domination number of the MGEO-P $(n, m, \alpha, \beta, p)$ model. The theorem poses that a sublinear bound exist on the domination number for OSNs.

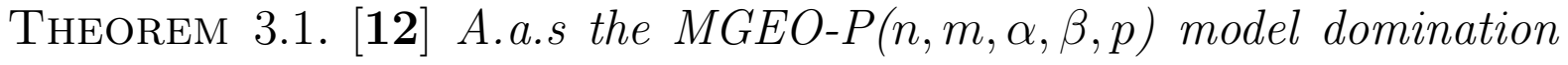
number satisfies

$$
\gamma(G)=\Omega\left(C^{-m /(1-\alpha)} n^{\alpha+\beta}\right) \text { and } \gamma(G)=O\left(n^{\alpha+\beta} \log n\right)
$$




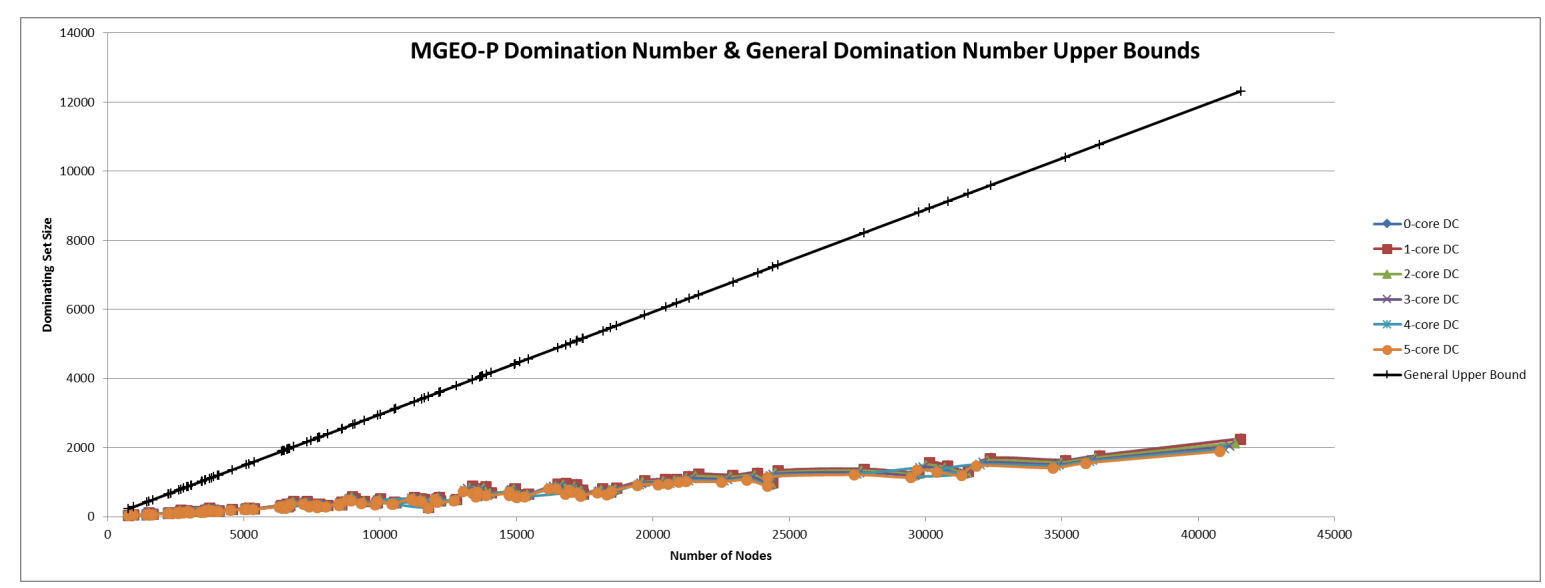

Figure 3.4. Domination number upper bounds of MGEO-P samples corresponding to the Facebook 100 graphs with Theorem 1.1 upper bound $(\delta=5)$.

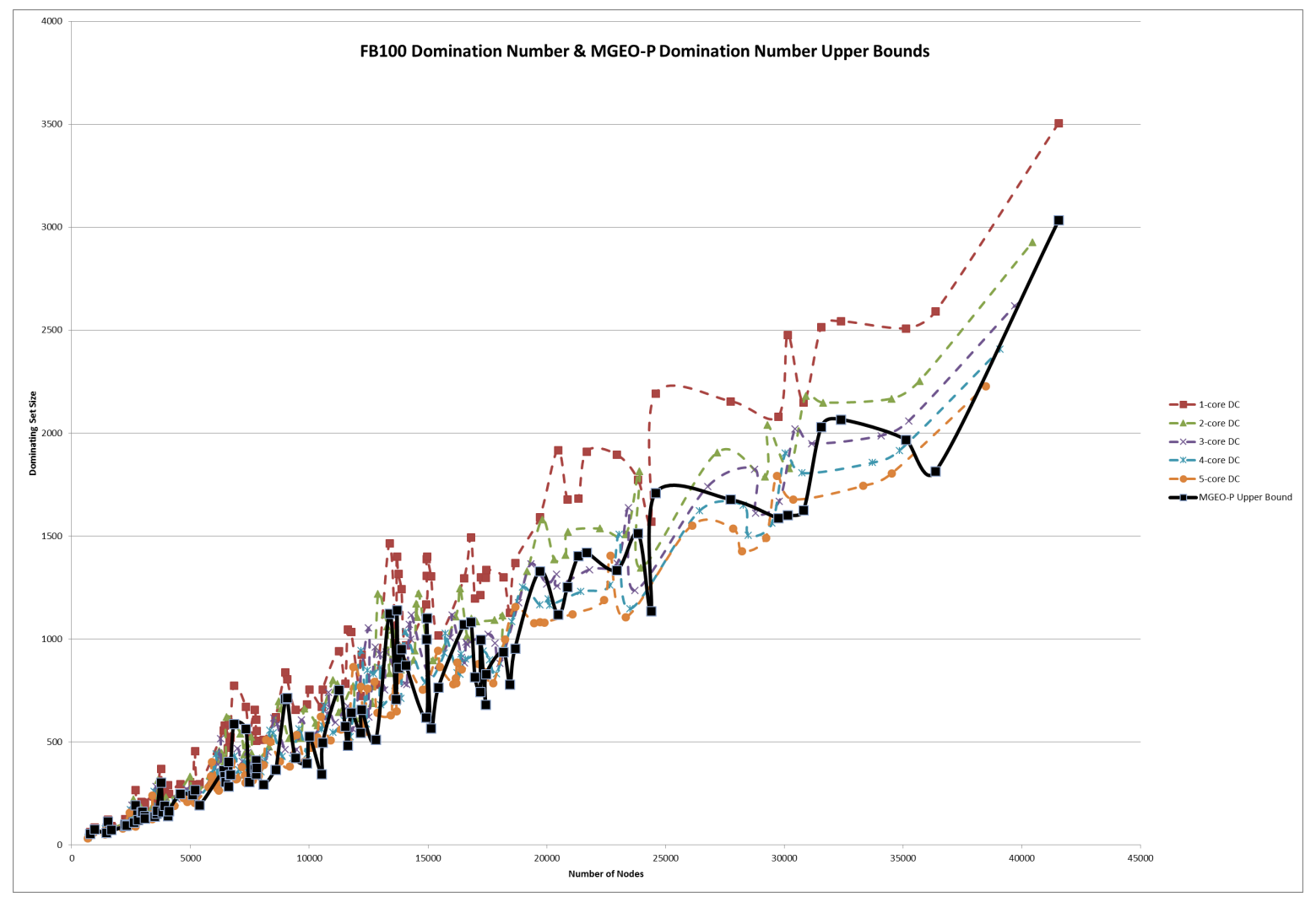

FiguRE 3.5. Domination number upper bounds of the Facebook 100 graphs with Theorem 3.1 upper bound, showing a very close correlation.

where $C$ is any constant greater than 6 and $m=o(\log n)$. Particularly, a.a.s. $\gamma(G)=n^{\alpha+\beta+o(1)}$. 
Based on a comparison of the plots, there is a high correlation between the theoretical MGEO-P upper bound and the domination number of the FB100 graphs. For the 100 samples, there is a noticeable pattern emerging. This provides empirical evidence that on-line social networks domination numbers follow sub-linear growth; more precisely:

$$
\gamma(G)=O\left(n^{\alpha+\beta} \log n\right) .
$$

By incorporating a sub-linear line of best fits using MATLAB, we will be able to see how well the data fits this type of growth. For simplicity, we ignore the constant in front of the big Oh term. From these trend lines, we can determine how correlated the sub-linear fit is with the data. Table 3.1 provides the coefficient of determination; that is, how close the data fits with the regression line.

There are high correlations even when taken the 5-core. The theoretical upper bound occurs asymptotically almost surely. As our data sets are relatively small order (that is, orders in the thousands rather than millions or billions), the asymptotic results predicted in Theorem 3.1 may not be fully evident in the MGEO-P samples. As more experiments are done with larger orders, we hope that their domination number demonstrates a more clear sub-linear growth and that the data fits more tightly. 


\begin{tabular}{|c|c|c|}
\hline$k$ & $x$ & $R^{2}$ \\
\hline 1 & 0.509 & 0.8472 \\
\hline 2 & 0.492 & 0.8292 \\
\hline 3 & 0.4818 & 0.8179 \\
\hline 4 & 0.4741 & 0.8093 \\
\hline 5 & 0.4677 & 0.803 \\
\hline
\end{tabular}

TABLE 3.1. Fitting $y=n^{x} \log n$ to the domination number of the k-cores of FB100, where $1 \leq k \leq 5$. 


\section{CHAPTER 4}

\section{Distance Memoryless Geometric Protean Model}

\subsection{Motivation}

The MGEO-P model has a probability parameter $p$ which was considered 1 during our simulations of the Facebook 100 graphs. Recall that Blau space predicts that agents with similar attributes are closer in the space. We abstract this to a new paradigm in the setting of MGEO-P, where vertices are adjacent in a region of influence with probability given by the function $f(D(u, v))$, with $u$ and $v$ distinct vertices. That is, $f$ is a function which depends on the metric distance between $u$ and $v$. The distance $D(u, v)$ is defined below. In particular, vertices have a higher probability of being adjacent to other vertices in their radius of influence which are closer. An example of this is friendships within a community. A person will more likely be friends with someone who lives geographically closer to them. A larger scale example would be Facebook friendships between two people from different countries, where one may suspect that such people have a lower probability of being friends. This allows distance to play the role of the probability parameter. The model that uses this as the function of $p$ is known as the distance memoryless geometric protean model, written DMGEO-P. This is new model will be the focus of this chapter. We define the model below in Section 4.2, and then give the results of simulations of the model in Section 4.3. 


\subsection{The Model}

The DMGEO-P model is defined in a similar way to the MGEO-P model, except instead of five parameters there are four parameters DMGEO$\mathrm{P}(n, m, \alpha, \beta)$. The parameters are exactly like the MGEO-P model: the number of nodes $n$, the dimension $m$, the attachment strength parameter $0<\alpha<1$ and the density parameter $0<\beta<1-\alpha$. The process is the exact same as the original MGEO-P model, however edges are added based on the following rule.

An undirected edge is created between $v$ and any pre-existing vertex $u$ with probability $f(D(u, v))$, if $D(v, u) \leq I\left(r_{u}\right)$. The distance and probability of adjacency, respectively are computed as follows:

$$
\begin{gathered}
D(u, v)=\min \left\{\left\|q_{v}-q_{u}-z\right\|_{\infty}: z \in\{-1,0,1\}^{m}\right\}, \\
f(D(u, v))=1-\frac{D(u, v)}{I\left(r_{u}\right)}
\end{gathered}
$$

where $\|\cdot\|_{\infty}$ is the infinity-norm.

Figure 4.1 demonstrates the process of simulating DMGEO-P $(3,2,0.17,0.27)$ which we explain here. The network initially starts out empty. In the first step, a node is placed with the coordinates $(0.1894,0.1284)$ and a radius of 0.431081. There are no pre-existing nodes to check for any edges. During the second step, another node is generated at $(0.7439,0.7232)$ and a computed radius of 0.406416 . The procedure continues with checking if the 
distance between the current generated node and any pre-existing node is less than the pre-existing node's radius. In this case, $D(u, v)=D(1,2)=$ $0.445500 \not \leq 0.431081$. Therefore, an edge is not placed between these two nodes. Finally, the third node is placed in the space at $(0.4200,0.9213)$ with radius 0.392648 . Repeating the process, computing the distance of nodes one and three $D(1,3)=0.2306 \leq 0.431081$ tells us an edge is placed with a probability of $f(D(1,3))=0.203034$. Likewise, for nodes two and three $D(2,3)=0.3239 \leq 0.406416$, thus, an edge is placed with a probability of $f(D(2,3))=0.465065$. Figure 4.2 shows visual representations of graph samples generated by the DMGEO-P model.

Like the MGEO-P samples, simulations were generated and a preliminary analysis was done. Tables A.29 A.30, A.31 provides us with the minimum degree, average degree, maximum degree and density of DMGEO-P samples analogous to the Facebook 100 graphs. When comparing these samples with the real data, the model tends to generate more sparse graphs with much less density and maximum degree. One might expect a domination number that is higher than the Facebook data. More nodes would be required to completely cover the network because the maximum degree is low. However, there are less graph samples with a minimum degree of one as in the Facebook data. And as in mentioned in Chapter 1, the domination set is sensitive to low degrees. We hope to see less "shifts" (that is, changes) in the domination set upper bound when taking the $k$-core of the DMGEO-P model. There is a higher probability of the low degree nodes being adjacent to at least one 


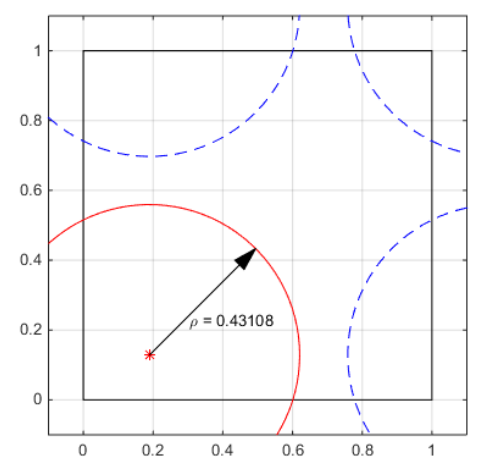

(A)

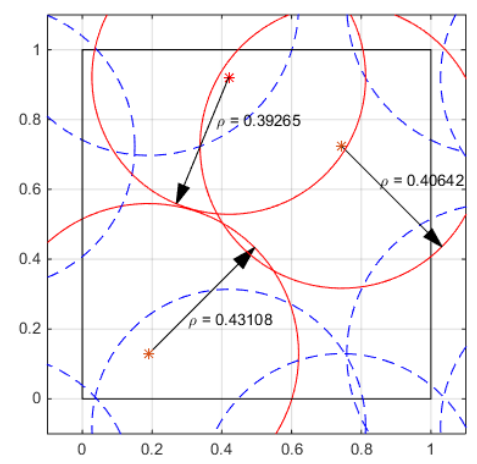

(c)

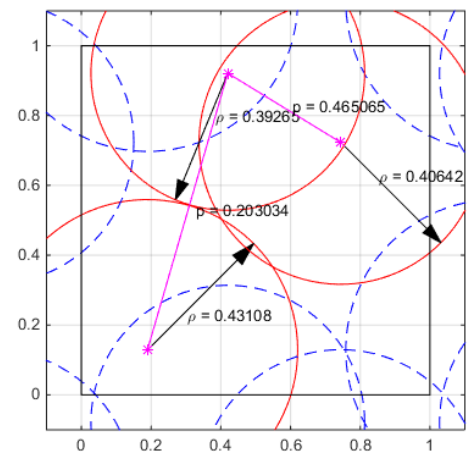

(E)

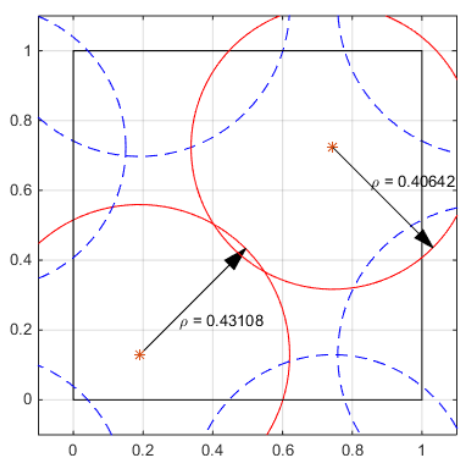

(в)

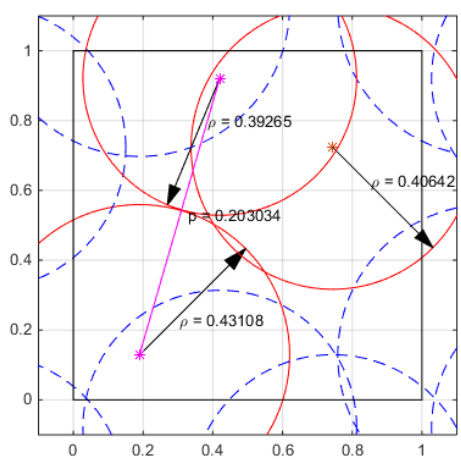

(D)

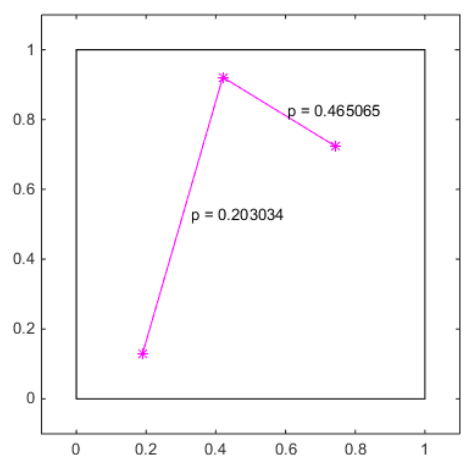

$(\mathrm{F})$

Figure 4.1. Time steps of a DMGEO-P $(3,2,0.17,0.27)$ sample. The unit square is given by the black interior rectangle from 0 to 1 . The broken blue circles are copies of nodes illustrating the torus metric. The images demonstrate the concept that the closer a node is to another in geometric space, there is a higher probability of adjacency. This is seen in the difference of probability between the two edges shown. (A) Time step 1: First node is added to the network. (B) Time step 2: Second node is added to the network. No nodes are overlapping, thus no edges. (C) Time step 3: Third node is added to the network. Overlaps with node one and two. (D) An edge is placed in between nodes one and three with probability of 0.203034 . (E) An edge is placed in between nodes two and three with probability of 0.465065 . (F) Final graph without node radii. 

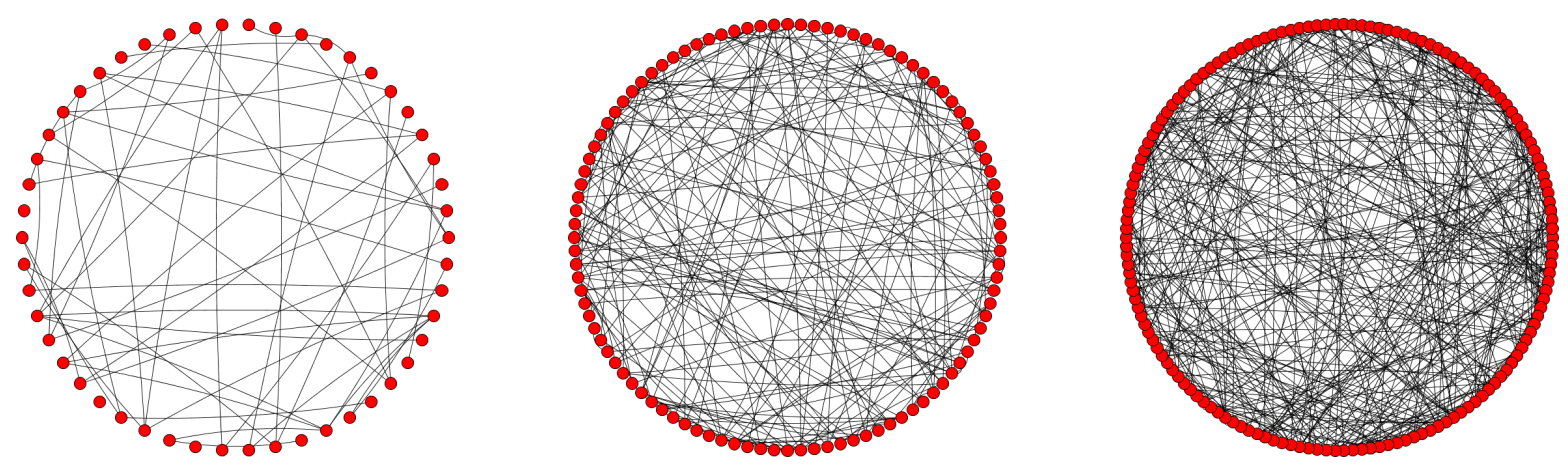

DMGEO-

$\mathrm{P}(50,3,0.17,0.27)$

DMGEO-

$\mathrm{P}(100,3,0.17,0.27)$

DMGEO-

$\mathrm{P}(150,3,0.17,0.27)$
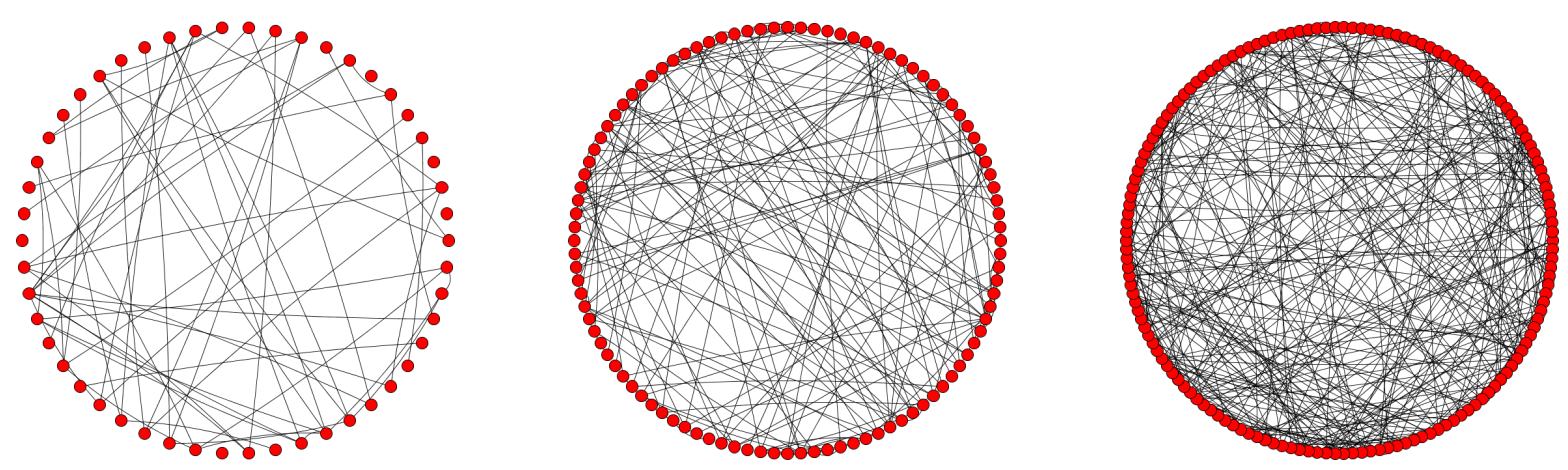

DMGEO-

$\mathrm{P}(50,4,0.17,0.27)$

DMGEO-

DMGEO-

$\mathrm{P}(100,4,0.17,0.27)$

$\mathrm{P}(150,4,0.17,0.27)$

Figure 4.2. Samples of the $\operatorname{DMGEO}-\mathrm{P}(n, m, \alpha, \beta)$ model, with various choices of parameters.

agent in the dominating set since the maximum degree of the samples are also quite low relative to the order of the graphs.
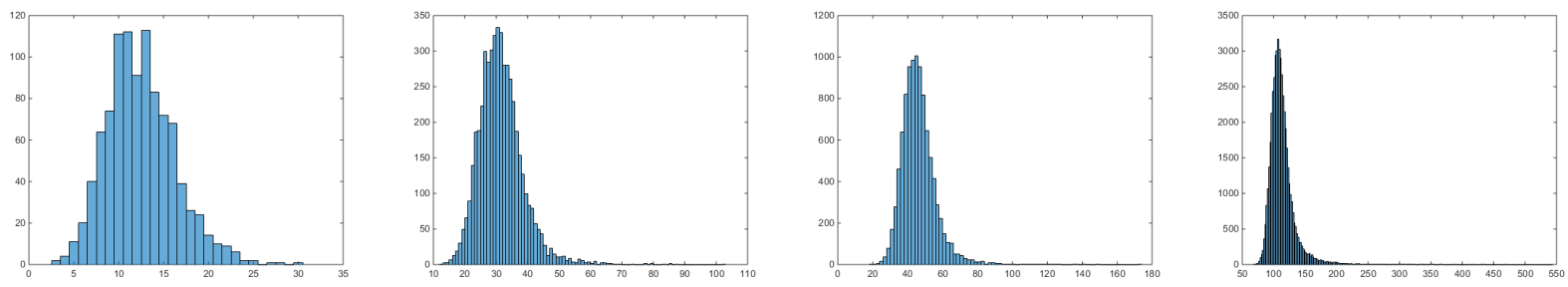

Figure 4.3. Degree distribution of DMGEO-P $(n, 4,0.17,0.27)$ samples for $n=1,000,5,000,10,000$ and 50,000. 

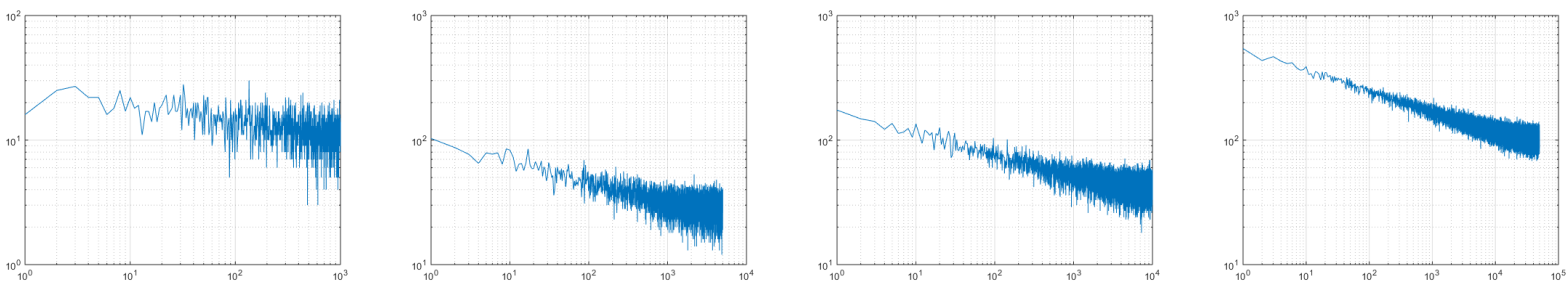

Figure 4.4. Log-log plots of $\operatorname{DMGEO}-\mathrm{P}(n, 4,0.17,0.27)$ samples for $n=$ $1,000,5,000,10,000$ and 50,000 . 


\subsection{Domination Number of the DMGEO-P Model}

Tables A.32, A.33, A.34, A.35, A.36, A.37 present the results of the DS-RAI algorithm for DMGEO-P samples that correspond to the FB100 network for their $k$-cores, where $1 \leq k \leq 5$. See Figure 4.5 for the plot of the dominating set size relative to the graph's order. Notice, as expected, the $k$-cores are all very similar; there is hardly any noticeable changes in domination set size. The DMGEO-P model appears to generate samples that are not as sensitive to domination noise (that is, the effect of having a higher domination number due to nodes with low degrees) than the MGEO-P model.

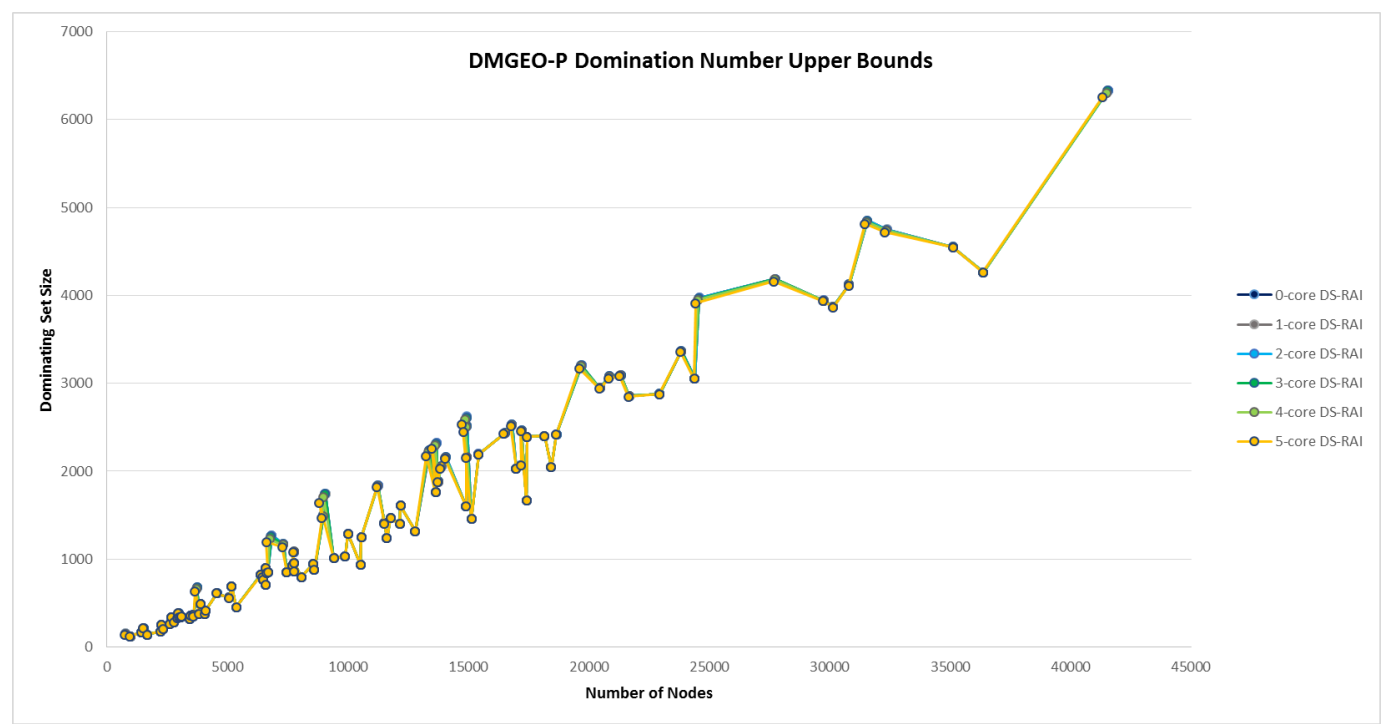

Figure 4.5. Domination number upper bounds of DMGEO-P samples corresponding to the Facebook 100 graphs using the DS-RAI algorithm.

Figure 4.6 compares the domination size of the DS-RAI algorithm for both DMGEO-P samples and the FB100 data. They appear to be relative close in size. It is interesting to observe the Facebook 100 graphs are more dense and should require less nodes in its domination set. As opposed to the DMGEO-P 
model where graphs are less dense and should require more nodes to cover the graph completely. However, both contains domination sets around the same size. Due to the higher minimum degree in the DMGEO-P model, there are a lower number of nodes with low degrees (when comparing the FB100 data). It would be advantageous to calculate the clustering coefficient of the Facebook 100, MGEO-P and DMGEO-P domination set nodes to see if any pattern or observations can be made about such nodes. We leave this for future work but hope to expect that these agents have a higher than average clustering coefficient.

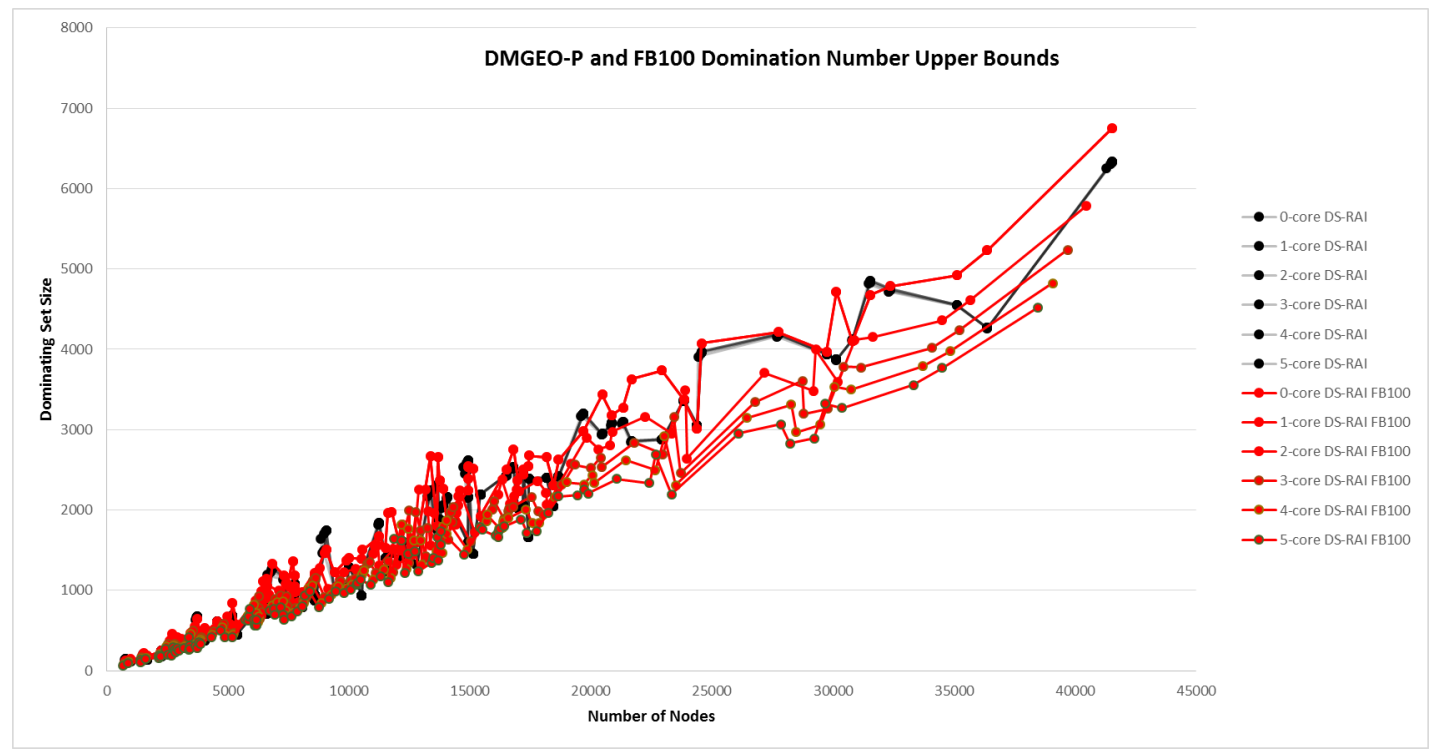

FIGURE 4.6. Domination number upper bounds of DMGEO-P samples corresponding to the Facebook 100 graphs and FB100.

Using the same Theorem 1.1 with the minimum degree of 5, Figure 4.7 shows its large overestimation on the domination size. All of our results are the same for Theorem 1.1. It shows a linear growth in domination number with order. Let us compare the MGEO-P theoretical domination number upper bound mentioned within $[\mathbf{1 2}]$ and the DMGEO-P domination size in 


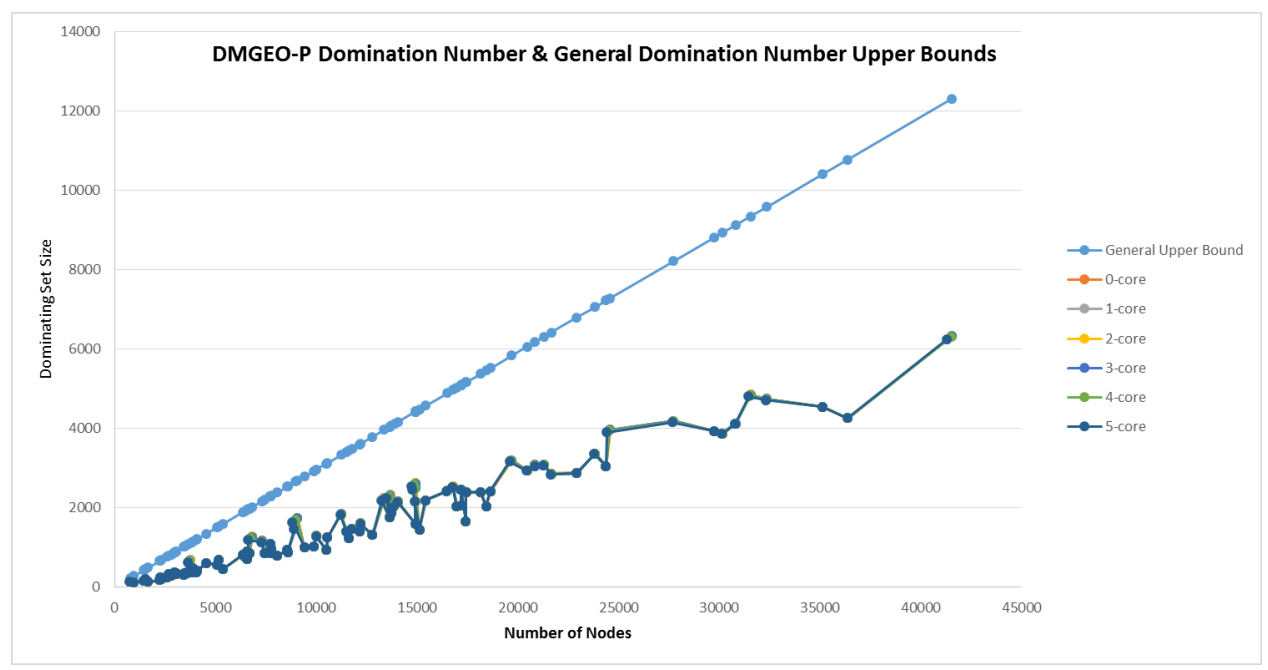

Figure 4.7. Domination number upper bounds of DMGEO-P samples corresponding to the Facebook 100 graphs with Theorem 1.1 upper bound $(\delta=5)$.

Figure 4.8. The resulting plots are similar in shape but not order. One should notice however that we only have the results of the DS-RAI algorithm. The DS-DC algorithm will result in a smaller set. An open question now is, does the DMGEO-P model follow Theorem 3.1? In particular, does the DMGEO$\mathrm{P}$ model follow a sub-linear domination number upper bound?

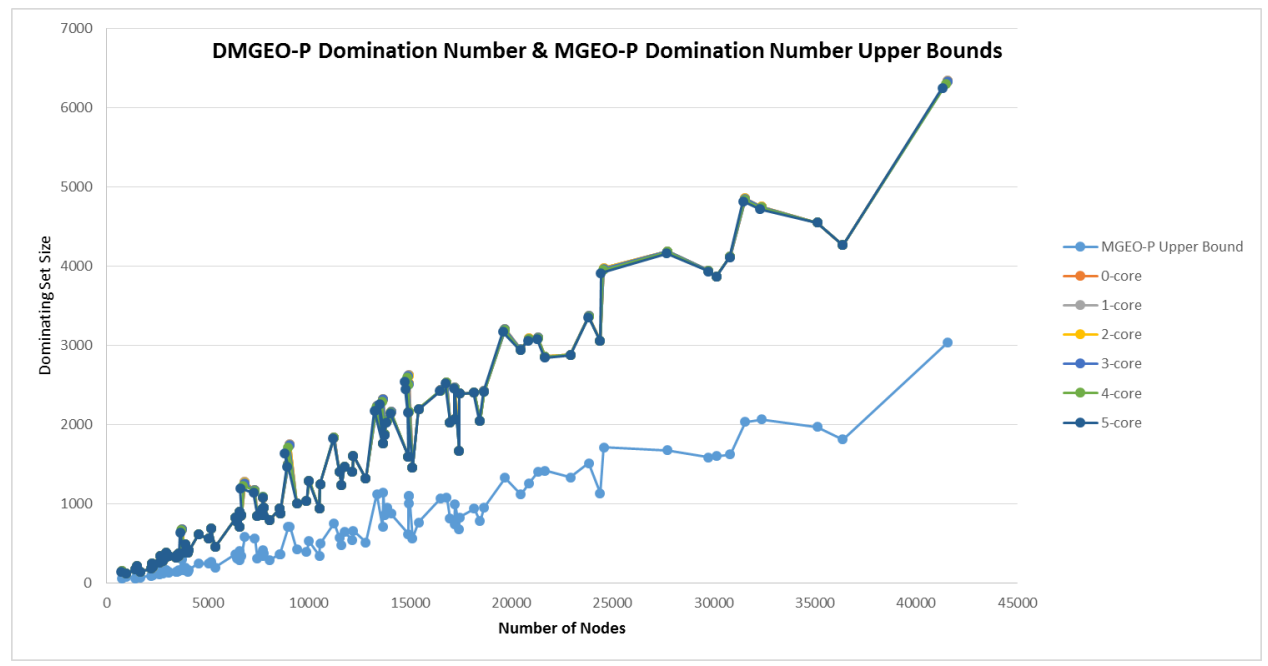

Figure 4.8. Domination number upper bounds of DMGEO-P samples corresponding to the Facebook 100 graphs with Theorem 1.1 upper bound $(\delta=5)$. 



\section{CHAPTER 5}

\section{Conclusions and Future Directions}

\subsection{Summary}

In Chapter 2, we investigated the MGEO-P model for properties found in OSNs. Theorems were stated for the model's average degree, diameter, and power-law degree distribution. We simulated graphs for the model, and confirmed experimentally that these satisfy power-law degree distributions.

In Chapter 3, we began with some brief background and analysis of the Facebook 100 dataset, and then went on to computing domination sets for the $k$-core, where $1 \leq k \leq 5$, using the DS-DC and DS-RAI algorithms. In [9], they investigated the FB100 graphs and computed matching MGEO$\mathrm{P}$ parameters for each institution within the set. With these parameters, MGEO-P samples were generated with their domination set computed using the DS-RAI and DS-DC algorithms. Recent theoretical results have suggested a sub-linear upper bound on the domination number in MGEO-P samples. We plotted the theoretical bound for the MGEO-P model using the parameters in $[\mathbf{9}]$, and the best estimation we have of the domination number for FB100 (from the DS-DC results). There is a correlation of the domination number of real Facebook datasets and graphs simulated from MGEO-P.

In our simulations of MGEO-P, we noticed that our samples did follow the theoretical bounds on the domination number. As mentioned in Chapter 3, 
the constant term of the big Oh bound was not considered in our plots. Also, we used the parameters for the MGEO-P matching the datasets found in $[\mathbf{9}]$, and then percolated our graphs to match the number of edges of the dataset. This possibly explains why our generated samples are more dense and why our domination sets are smaller than the plotted function $f(n)=n^{\alpha+\beta}$.

In Chapter 4, the MGEO-P model was modified to incorporate a more geometric view, better approximating Blau space. Here we found that these sample's domination number follow closely along with the actual Facebook data and MGEO-P theoretical domination number upper bound. Some investigation should be done to explain why the DMGEO-P model is generating less dense graphs yet have a domination number that more closely resembles dense OSNs such as FB100.

\subsection{Open Problems}

There is much more work to be done in this area of research. Completing the DS-DC algorithm for the DMGEO-P samples will help us deduce if the model follows OSNs domination numbers. One can perform additional domination set algorithms to find the actual domination number of the Facebook 100 dataset. The ultimate goal would be to apply an algorithm for computing the exact domination number of OSNs (such algorithm is mentioned in $[\mathbf{4 2}]$ ). One can look into elite members (that is, influential members in the social network) of networks and see what role they play in dominating sets. In addition, dominating sets can be widely used in "directors network"

as a niche area in Corporate Finance. The PageRank of each network should 
be computed, and the agents of the institution's domination set should be crossed referenced to see if there is any correlation with the agents of high PageRank and agents within the domination set. As mentioned in Chapter 4, the clustering coefficients for each graph should be computed and analyzed to see any patterns between the dominating agents and their clustering coefficient.

Another, possibly more complex problem that should be studied, is to consider models where the sphere of influence is not a ball in the corresponding metric space. The idea behind this is agents do not have a uniform radius of influence in $m$-dimensions, and the radius would depend on what dimension is being considered (for example, socio-demographic characteristics) for that agent. For example, if one dimension was income and another community impact, there can be many users with low level of income and high community impact, and vice versa.

Another important consideration to consider is the domination number in other OSNs datasets. Data from other on-line social networks (such as LinkedIn and Twitter) should all be studied to determine if they follow the theoretical MGEO-P domination number upper bound. If this is true, then the MGEO-P model will be a sound model to simulate both OSN properties and the domination number.

Another question is to determine why agents belong to dominating sets. There might be unique properties about these agents which can play an important role in OSNs. Learning and understanding how agents join or 
leave dominating sets may allow us to control future network behaviour. These agents are informally the glue of the network, they have the most spread and value in network information diffusion.

In summary, the MGEO-P follows properties of OSNs and follows their domination number upper bound. Work in the thesis provides experimental evidence that MGEO-P samples do follow theoretical predicted values of dominating sets, and that OSNs follow the MGEO-P sub-linear domination number bound. In addition to introducing a modified model of the MGEO-P, samples of the DMGEO-P model possesses domination number with order similar to that found in OSNs. 


\section{APPENDIX A}

\section{Data Tables}

\section{A.1. MGEO-P Data Tables}

\begin{tabular}{|c|c|c|c|c|c|c|}
\hline Dataset Name & Nodes & Edges & Min Degree & Avg Degree & Max Degree & Density \\
\hline Caltech36 & 769 & 16656 & 1 & 43 & 120 & 0.056 \\
\hline Reed98 & 962 & 18812 & 0 & 39 & 228 & 0.041 \\
\hline Haverford76 & 1446 & 59589 & 0 & 82 & 205 & 0.057 \\
\hline Simmons81 & 1518 & 32988 & 0 & 43 & 195 & 0.029 \\
\hline Swarthmore42 & 1659 & 61050 & 1 & 74 & 298 & 0.044 \\
\hline Amherst41 & 2235 & 90954 & 1 & 81 & 333 & 0.036 \\
\hline Bowdoin47 & 2252 & 84387 & 1 & 75 & 282 & 0.033 \\
\hline Hamilton46 & 2314 & 96394 & 1 & 83 & 429 & 0.036 \\
\hline Trinity100 & 2613 & 111996 & 0 & 86 & 311 & 0.033 \\
\hline USFCA72 & 2682 & 65252 & 0 & 49 & 378 & 0.018 \\
\hline Williams40 & 2790 & 112986 & 1 & 81 & 368 & 0.029 \\
\hline Oberlin44 & 2920 & 89912 & 0 & 62 & 339 & 0.021 \\
\hline Smith60 & 2970 & 97133 & 1 & 65 & 234 & 0.022 \\
\hline Wellesley22 & 2970 & 94899 & 0 & 64 & 340 & 0.022 \\
\hline Vassar85 & 3068 & 119161 & 1 & 78 & 355 & 0.025 \\
\hline Middlebury45 & 3075 & 124610 & 0 & 81 & 291 & 0.026 \\
\hline Pepperdine86 & 3445 & 152007 & 0 & 88 & 386 & 0.026 \\
\hline Colgate88 & 3482 & 155043 & 1 & 89 & 409 & 0.026 \\
\hline Santa74 & 3578 & 151747 & 0 & 85 & 375 & 0.024 \\
\hline Wesleyan43 & 3593 & 138035 & 0 & 77 & 427 & 0.021 \\
\hline Mich67 & 3748 & 81903 & 1 & 44 & 228 & 0.012 \\
\hline Bucknell39 & 3826 & 158864 & 0 & 83 & 354 & 0.022 \\
\hline Brandeis99 & 3898 & 137567 & 0 & 71 & 368 & 0.018 \\
\hline Howard90 & 4047 & 204850 & 0 & 101 & 382 & 0.025 \\
\hline Rice31 & 4087 & 184828 & 0 & 90 & 400 & 0.022 \\
\hline Rochester38 & 4563 & 161404 & 0 & 71 & 275 & 0.016 \\
\hline Lehigh96 & 5075 & 198347 & 1 & 78 & 351 & 0.015 \\
\hline Johns-Hopkins55 & 5180 & 186586 & 0 & 72 & 363 & 0.014 \\
\hline Wake73 & 5372 & 279191 & 1 & 104 & 447 & 0.019 \\
\hline American75 & 6386 & 217662 & 0 & 68 & 561 & 0.011 \\
\hline MIT8 & 6440 & 251252 & 0 & 78 & 379 & 0.012 \\
\hline TaBw5 A.1 & G & 0 & & & \\
\hline
\end{tabular}

TABLE A.1. General graph properties computed for MGEO-P samples of Facebook 100. 


\begin{tabular}{|c|c|c|c|c|c|c|}
\hline Dataset Name & Nodes & Edges & Min Degree & Avg Degree & Max Degree & Density \\
\hline William77 & 6472 & 266378 & 0 & 82 & 490 & 0.013 \\
\hline UChicago30 & 6591 & 208103 & 0 & 63 & 508 & 0.010 \\
\hline Princeton12 & 6596 & 293320 & 0 & 89 & 487 & 0.013 \\
\hline Carnegie49 & 6637 & 249967 & 0 & 75 & 454 & 0.011 \\
\hline Tufts18 & 6682 & 249728 & 0 & 75 & 355 & 0.011 \\
\hline UC64 & 6833 & 155332 & 0 & 45 & 205 & 0.007 \\
\hline Vermont70 & 7324 & 191221 & 0 & 52 & 374 & 0.007 \\
\hline Emory27 & 7460 & 330014 & 0 & 88 & 448 & 0.012 \\
\hline Dartmouth6 & 7694 & 304076 & 1 & 79 & 436 & 0.010 \\
\hline Tulane29 & 7752 & 283918 & 1 & 73 & 291 & 0.009 \\
\hline WashU32 & 7755 & 367541 & 0 & 95 & 476 & 0.012 \\
\hline Villanova62 & 7772 & 314989 & 0 & 81 & 444 & 0.010 \\
\hline Vanderbilt48 & 8069 & 427832 & 0 & 106 & 531 & 0.013 \\
\hline Yale4 & 8578 & 405450 & 1 & 95 & 547 & 0.011 \\
\hline Brown11 & 8600 & 384526 & 0 & 89 & 672 & 0.010 \\
\hline UCSC68 & 8991 & 224584 & 0 & 50 & 285 & 0.006 \\
\hline Maine59 & 9069 & 243247 & 0 & 54 & 429 & 0.006 \\
\hline Georgetown15 & 9414 & 425638 & 0 & 90 & 619 & 0.010 \\
\hline Duke14 & 9895 & 506442 & 0 & 102 & 521 & 0.010 \\
\hline Bingham82 & 10004 & 362894 & 0 & 73 & 419 & 0.007 \\
\hline Mississippi66 & 10521 & 610911 & 0 & 116 & 744 & 0.011 \\
\hline Northwestern25 & 10567 & 488337 & 1 & 92 & 435 & 0.009 \\
\hline Cal65 & 11247 & 351358 & 1 & 62 & 219 & 0.006 \\
\hline BC17 & 11509 & 486967 & 0 & 85 & 505 & 0.007 \\
\hline Stanford3 & 11621 & 568330 & 1 & 98 & 516 & 0.008 \\
\hline Columbia2 & 11770 & 444333 & 1 & 76 & 804 & 0.006 \\
\hline Notre-Dame57 & 12155 & 541339 & 0 & 89 & 628 & 0.007 \\
\hline GWU54 & 12193 & 469528 & 1 & 77 & 761 & 0.006 \\
\hline Baylor93 & 12803 & 679817 & 1 & 106 & 471 & 0.008 \\
\hline USF51 & 13377 & 321214 & 0 & 48 & 351 & 0.004 \\
\hline Syracuse56 & 13653 & 543982 & 0 & 80 & 497 & 0.006 \\
\hline Temple83 & 13686 & 360795 & 0 & 53 & 676 & 0.004 \\
\hline UC61 & 13746 & 442174 & 0 & 64 & 402 & 0.005 \\
\hline Northeastern19 & 13882 & 381934 & 0 & 55 & 482 & 0.004 \\
\hline JMU79 & 14070 & 485564 & 0 & 69 & 548 & 0.005 \\
\hline UPenn7 & 14916 & 686501 & 0 & 92 & 536 & 0.006 \\
\hline UCSB37 & 14935 & 482224 & 0 & 65 & 386 & 0.004 \\
\hline UCF52 & 14940 & 428989 & 0 & 57 & 318 & 0.004 \\
\hline UCSD34 & 14948 & 443221 & 0 & 59 & 392 & 0.004 \\
\hline Harvard1 & 15126 & 824617 & 0 & 109 & 608 & 0.007 \\
\hline
\end{tabular}

TABLE A.2. General graph properties computed for MGEO-P samples of Facebook 100. 


\begin{tabular}{|c|c|c|c|c|c|c|}
\hline Dataset Name & Nodes & Edges & Min Degree & Avg Degree & Max Degree & Density \\
\hline MU78 & 15436 & 649449 & 0 & 84 & 456 & 0.005 \\
\hline UMass92 & 16516 & 519385 & 0 & 63 & 521 & 0.004 \\
\hline UC33 & 16808 & 522147 & 0 & 62 & 381 & 0.004 \\
\hline Tennessee95 & 16979 & 770659 & 0 & 91 & 779 & 0.005 \\
\hline UVA16 & 17196 & 789321 & 1 & 92 & 549 & 0.005 \\
\hline UConn91 & 17212 & 604870 & 0 & 70 & 469 & 0.004 \\
\hline Oklahoma97 & 17425 & 892528 & 0 & 102 & 656 & 0.006 \\
\hline USC35 & 17444 & 801853 & 0 & 92 & 467 & 0.005 \\
\hline UNC28 & 18163 & 766800 & 0 & 84 & 901 & 0.005 \\
\hline Auburn71 & 18448 & 973918 & 0 & 106 & 581 & 0.006 \\
\hline Cornell5 & 18660 & 790777 & 0 & 85 & 730 & 0.005 \\
\hline BU10 & 19700 & 637528 & 0 & 65 & 360 & 0.003 \\
\hline UCLA26 & 20467 & 747613 & 0 & 73 & 448 & 0.004 \\
\hline Maryland58 & 20871 & 744862 & 0 & 71 & 628 & 0.003 \\
\hline Virginia63 & 21325 & 698178 & 0 & 65 & 501 & 0.003 \\
\hline NYU9 & 21679 & 715715 & 0 & 66 & 1173 & 0.003 \\
\hline Berkeley13 & 22937 & 852444 & 0 & 74 & 916 & 0.003 \\
\hline Wisconsin 87 & 23842 & 835952 & 0 & 70 & 841 & 0.003 \\
\hline UGA50 & 24389 & 1174057 & 1 & 96 & 572 & 0.004 \\
\hline Rutgers89 & 24580 & 784602 & 0 & 64 & 459 & 0.003 \\
\hline FSU53 & 27737 & 1034802 & 0 & 75 & 526 & 0.003 \\
\hline Indiana69 & 29747 & 1305765 & 0 & 88 & 620 & 0.003 \\
\hline Michigan23 & 30147 & 1176516 & 0 & 78 & 633 & 0.003 \\
\hline UIllinois20 & 30809 & 1264428 & 0 & 82 & 459 & 0.003 \\
\hline Texas80 & 31560 & 1219650 & 0 & 77 & 603 & 0.002 \\
\hline MSU24 & 32375 & 1118774 & 0 & 69 & 616 & 0.002 \\
\hline UF21 & 35123 & 1465660 & 0 & 83 & 680 & 0.002 \\
\hline Texas84 & 36371 & 1590655 & 0 & 87 & 802 & 0.002 \\
\hline Penn94 & 41554 & 1362229 & 0 & 66 & 986 & 0.002 \\
\hline
\end{tabular}

TABLE A.3. General graph properties computed for MGEO-P samples of Facebook 100. 


\begin{tabular}{|c|c|}
\hline \multicolumn{2}{|c|}{ 0-core } \\
\hline Nodes & DS-RAI \\
\hline 769 & 74 \\
\hline 962 & 117 \\
\hline 1446 & 98 \\
\hline 1518 & 171 \\
\hline 1659 & 149 \\
\hline 2235 & 208 \\
\hline 2252 & 206 \\
\hline 2314 & 206 \\
\hline 2613 & 200 \\
\hline 2682 & 343 \\
\hline 2790 & 248 \\
\hline 2920 & 304 \\
\hline 2970 & 302 \\
\hline 2970 & 253 \\
\hline 3068 & 254 \\
\hline 3075 & 257 \\
\hline 3445 & 311 \\
\hline 3482 & 276 \\
\hline 3578 & 284 \\
\hline 3593 & 359 \\
\hline 3748 & 429 \\
\hline 3826 & 363 \\
\hline 3898 & 359 \\
\hline 4047 & 325 \\
\hline 4087 & 308 \\
\hline
\end{tabular}

\begin{tabular}{|c|c|}
\hline \multicolumn{2}{|c|}{ 0-core } \\
\hline Nodes & DS-RAI \\
\hline 4563 & 376 \\
\hline 5075 & 394 \\
\hline 5180 & 427 \\
\hline 5372 & 419 \\
\hline 6386 & 609 \\
\hline 6440 & 516 \\
\hline 6472 & 559 \\
\hline 6591 & 644 \\
\hline 6596 & 547 \\
\hline 6637 & 582 \\
\hline 6682 & 576 \\
\hline 6833 & 697 \\
\hline 7324 & 774 \\
\hline 7460 & 620 \\
\hline 7694 & 663 \\
\hline 7752 & 614 \\
\hline 7755 & 559 \\
\hline 7772 & 641 \\
\hline 8069 & 598 \\
\hline 8578 & 630 \\
\hline 8600 & 804 \\
\hline 8991 & 995 \\
\hline 9069 & 777 \\
\hline 9414 & 824 \\
\hline 9895 & 686 \\
\hline
\end{tabular}

\begin{tabular}{|c|c|}
\hline \multicolumn{2}{|c|}{0 -core } \\
\hline Nodes & DS-RAI \\
\hline 10004 & 903 \\
\hline 10521 & 809 \\
\hline 10567 & 717 \\
\hline 11247 & 874 \\
\hline 11509 & 919 \\
\hline 11621 & 885 \\
\hline 11770 & 515 \\
\hline 12155 & 1068 \\
\hline 12193 & 835 \\
\hline 12803 & 908 \\
\hline 13377 & 1531 \\
\hline 13653 & 1111 \\
\hline 13686 & 1423 \\
\hline 13746 & 1371 \\
\hline 13882 & 1599 \\
\hline 14070 & 1182 \\
\hline 14916 & 1267 \\
\hline 14935 & 1327 \\
\hline 14940 & 1338 \\
\hline 14948 & 1347 \\
\hline 15126 & 1160 \\
\hline 15436 & 1066 \\
\hline 16516 & 1749 \\
\hline 16808 & 1769 \\
\hline 16979 & 1348 \\
\hline & 2 \\
\hline
\end{tabular}

\begin{tabular}{|c|c|}
\hline \multicolumn{2}{|c|}{0 -core } \\
\hline Nodes & DS-RAI \\
\hline 17196 & 1420 \\
\hline 17212 & 1613 \\
\hline 17425 & 1493 \\
\hline 17444 & 1079 \\
\hline 18163 & 1435 \\
\hline 18448 & 1292 \\
\hline 18660 & 1457 \\
\hline 19700 & 1751 \\
\hline 20467 & 1870 \\
\hline 20871 & 1832 \\
\hline 21325 & 2165 \\
\hline 21679 & 2348 \\
\hline 22937 & 2307 \\
\hline 23842 & 2231 \\
\hline 24389 & 1665 \\
\hline 24580 & 2187 \\
\hline 27737 & 2252 \\
\hline 29747 & 2225 \\
\hline 30147 & 2894 \\
\hline 30809 & 2613 \\
\hline 31560 & 2119 \\
\hline 32375 & 2987 \\
\hline 35123 & 3007 \\
\hline 36371 & 3320 \\
\hline 41554 & 4116 \\
\hline
\end{tabular}

TABLE A.4. Dominating set sizes from the DS-RAI algorithm for the 0-core of MGEO-P samples of the Facebook 100 network. 


\begin{tabular}{|c|c|}
\hline \multicolumn{2}{|c|}{ 1-core } \\
\hline Nodes & DS-RAI \\
\hline 769 & 74 \\
\hline 961 & 119 \\
\hline 1445 & 97 \\
\hline 1517 & 170 \\
\hline 1659 & 149 \\
\hline 2235 & 210 \\
\hline 2252 & 210 \\
\hline 2314 & 206 \\
\hline 2612 & 199 \\
\hline 2679 & 339 \\
\hline 2790 & 257 \\
\hline 2919 & 305 \\
\hline 2969 & 301 \\
\hline 2970 & 253 \\
\hline 3068 & 256 \\
\hline 3074 & 258 \\
\hline 3444 & 299 \\
\hline 3482 & 276 \\
\hline 3577 & 281 \\
\hline 3592 & 356 \\
\hline 3748 & 434 \\
\hline 3825 & 359 \\
\hline 3894 & 355 \\
\hline 4045 & 323 \\
\hline 4085 & 314 \\
\hline
\end{tabular}

\begin{tabular}{|c|c|}
\hline \multicolumn{2}{|c|}{1 -core } \\
\hline Nodes & DS-RAI \\
\hline 4562 & 375 \\
\hline 5075 & 398 \\
\hline 5179 & 421 \\
\hline 5372 & 419 \\
\hline 6384 & 603 \\
\hline 6439 & 507 \\
\hline 6471 & 552 \\
\hline 6590 & 641 \\
\hline 6595 & 550 \\
\hline 6635 & 580 \\
\hline 6680 & 571 \\
\hline 6828 & 695 \\
\hline 7323 & 775 \\
\hline 7456 & 618 \\
\hline 7694 & 663 \\
\hline 7752 & 603 \\
\hline 7754 & 547 \\
\hline 7771 & 642 \\
\hline 8066 & 603 \\
\hline 8578 & 630 \\
\hline 8595 & 799 \\
\hline 8988 & 986 \\
\hline 9065 & 770 \\
\hline 9411 & 819 \\
\hline 9893 & 684 \\
\hline
\end{tabular}

\begin{tabular}{|c|c|}
\hline \multicolumn{2}{|c|}{1 -core } \\
\hline Nodes & DS-RAI \\
\hline 10001 & 900 \\
\hline 10520 & 808 \\
\hline 10567 & 730 \\
\hline 11247 & 882 \\
\hline 11508 & 922 \\
\hline 11621 & 884 \\
\hline 11770 & 499 \\
\hline 12151 & 1051 \\
\hline 12193 & 835 \\
\hline 12803 & 908 \\
\hline 13374 & 1538 \\
\hline 13650 & 1110 \\
\hline 13682 & 1418 \\
\hline 13738 & 1366 \\
\hline 13874 & 1586 \\
\hline 14064 & 1177 \\
\hline 14908 & 1257 \\
\hline 14930 & 1316 \\
\hline 14938 & 1337 \\
\hline 14944 & 1347 \\
\hline 15121 & 1157 \\
\hline 15435 & 1078 \\
\hline 16508 & 1734 \\
\hline 16801 & 1760 \\
\hline 16977 & 1345 \\
\hline $4 h 0$ & \\
\hline
\end{tabular}

\begin{tabular}{|c|c|}
\hline \multicolumn{2}{|c|}{1 -core } \\
\hline Nodes & DS-RAI \\
\hline 17196 & 1410 \\
\hline 17201 & 1615 \\
\hline 17422 & 1484 \\
\hline 17443 & 1076 \\
\hline 18160 & 1441 \\
\hline 18446 & 1292 \\
\hline 18658 & 1465 \\
\hline 19696 & 1748 \\
\hline 20459 & 1857 \\
\hline 20866 & 1815 \\
\hline 21316 & 2152 \\
\hline 21674 & 2335 \\
\hline 22930 & 2299 \\
\hline 23828 & 2202 \\
\hline 24389 & 1666 \\
\hline 24577 & 2160 \\
\hline 27733 & 2241 \\
\hline 29740 & 2215 \\
\hline 30135 & 2880 \\
\hline 30799 & 2616 \\
\hline 31558 & 2127 \\
\hline 32369 & 2995 \\
\hline 35115 & 3006 \\
\hline 36361 & 3315 \\
\hline 41538 & 4107 \\
\hline
\end{tabular}

TABLE A.5. Dominating set sizes from the DS-RAI algorithm for the 1-core of MGEO-P samples of the Facebook 100 network. 


\begin{tabular}{|c|c|}
\hline \multicolumn{2}{|c|}{2 -core } \\
\hline Nodes & DS-RAI \\
\hline 766 & 72 \\
\hline 957 & 115 \\
\hline 1443 & 95 \\
\hline 1509 & 162 \\
\hline 1653 & 145 \\
\hline 2227 & 200 \\
\hline 2248 & 203 \\
\hline 2306 & 198 \\
\hline 2607 & 194 \\
\hline 2662 & 323 \\
\hline 2783 & 244 \\
\hline 2902 & 289 \\
\hline 2960 & 293 \\
\hline 2961 & 244 \\
\hline 3054 & 242 \\
\hline 3069 & 249 \\
\hline 3433 & 288 \\
\hline 3472 & 266 \\
\hline 3563 & 267 \\
\hline 3578 & 344 \\
\hline 3719 & 405 \\
\hline 3813 & 348 \\
\hline 3877 & 339 \\
\hline 4032 & 311 \\
\hline 4080 & 302 \\
\hline
\end{tabular}

\begin{tabular}{|c|c|}
\hline \multicolumn{2}{|c|}{ 2-core } \\
\hline Nodes & DS-RAI \\
\hline 4547 & 361 \\
\hline 5063 & 385 \\
\hline 5162 & 409 \\
\hline 5358 & 410 \\
\hline 6366 & 589 \\
\hline 6426 & 495 \\
\hline 6442 & 525 \\
\hline 6555 & 607 \\
\hline 6575 & 524 \\
\hline 6618 & 565 \\
\hline 6661 & 553 \\
\hline 6793 & 653 \\
\hline 7276 & 724 \\
\hline 7433 & 596 \\
\hline 7667 & 626 \\
\hline 7735 & 591 \\
\hline 7738 & 531 \\
\hline 7745 & 619 \\
\hline 8047 & 577 \\
\hline 8554 & 759 \\
\hline 8559 & 615 \\
\hline 8947 & 961 \\
\hline 9035 & 750 \\
\hline 9385 & 793 \\
\hline 9868 & 659 \\
\hline
\end{tabular}

\begin{tabular}{|c|c|}
\hline \multicolumn{2}{|c|}{2 -core } \\
\hline Nodes & DS-RAI \\
\hline 9967 & 868 \\
\hline 10504 & 793 \\
\hline 10544 & 693 \\
\hline 11218 & 850 \\
\hline 11479 & 898 \\
\hline 11584 & 845 \\
\hline 11759 & 487 \\
\hline 12117 & 1019 \\
\hline 12170 & 812 \\
\hline 12778 & 878 \\
\hline 13280 & 1450 \\
\hline 13608 & 1356 \\
\hline 13617 & 1073 \\
\hline 13697 & 1327 \\
\hline 13799 & 1522 \\
\hline 14008 & 1117 \\
\hline 14870 & 1264 \\
\hline 14872 & 1220 \\
\hline 14885 & 1281 \\
\hline 14886 & 1292 \\
\hline 15086 & 1122 \\
\hline 15394 & 1029 \\
\hline 16428 & 1668 \\
\hline 16709 & 1685 \\
\hline 16938 & 1312 \\
\hline
\end{tabular}

\begin{tabular}{|c|c|}
\hline \multicolumn{2}{|c|}{2 -core } \\
\hline Nodes & DS-RAI \\
\hline 17126 & 1536 \\
\hline 17152 & 1361 \\
\hline 17375 & 1437 \\
\hline 17415 & 1046 \\
\hline 18102 & 1384 \\
\hline 18418 & 1274 \\
\hline 18607 & 1409 \\
\hline 19620 & 1678 \\
\hline 20376 & 1770 \\
\hline 20792 & 1763 \\
\hline 21223 & 2070 \\
\hline 21551 & 2230 \\
\hline 22839 & 2205 \\
\hline 23723 & 2110 \\
\hline 24334 & 1610 \\
\hline 24476 & 2061 \\
\hline 27648 & 2152 \\
\hline 29678 & 2152 \\
\hline 30020 & 2775 \\
\hline 30707 & 2510 \\
\hline 31493 & 2056 \\
\hline 32256 & 2906 \\
\hline 34989 & 2908 \\
\hline 36233 & 3185 \\
\hline 41344 & 3928 \\
\hline
\end{tabular}

TABLE A.6. Dominating set sizes from the DS-RAI algorithm for the 2-core of MGEO-P samples of the Facebook 100 network. 


\begin{tabular}{|c|c|}
\hline \multicolumn{2}{|c|}{3 -core } \\
\hline Nodes & DS-RAI \\
\hline 761 & 67 \\
\hline 947 & 105 \\
\hline 1442 & 94 \\
\hline 1494 & 150 \\
\hline 1649 & 142 \\
\hline 2222 & 195 \\
\hline 2239 & 197 \\
\hline 2298 & 193 \\
\hline 2605 & 192 \\
\hline 2632 & 296 \\
\hline 2771 & 229 \\
\hline 2887 & 275 \\
\hline 2945 & 284 \\
\hline 2948 & 231 \\
\hline 3039 & 226 \\
\hline 3057 & 237 \\
\hline 3419 & 288 \\
\hline 3462 & 257 \\
\hline 3552 & 257 \\
\hline 3563 & 332 \\
\hline 3695 & 381 \\
\hline 3806 & 342 \\
\hline 3856 & 320 \\
\hline 4021 & 300 \\
\hline 4071 & 303 \\
\hline
\end{tabular}

\begin{tabular}{|c|c|}
\hline \multicolumn{2}{|c|}{3 -core } \\
\hline Nodes & DS-RAI \\
\hline 4537 & 351 \\
\hline 5047 & 371 \\
\hline 5148 & 394 \\
\hline 5345 & 394 \\
\hline 6337 & 568 \\
\hline 6412 & 488 \\
\hline 6424 & 511 \\
\hline 6518 & 573 \\
\hline 6552 & 508 \\
\hline 6594 & 543 \\
\hline 6634 & 533 \\
\hline 6764 & 640 \\
\hline 7249 & 700 \\
\hline 7420 & 586 \\
\hline 7641 & 614 \\
\hline 7715 & 577 \\
\hline 7722 & 522 \\
\hline 7724 & 595 \\
\hline 8030 & 566 \\
\hline 8527 & 735 \\
\hline 8545 & 599 \\
\hline 8891 & 906 \\
\hline 8999 & 713 \\
\hline 9358 & 770 \\
\hline 9850 & 643 \\
\hline
\end{tabular}

\begin{tabular}{|c|c|}
\hline \multicolumn{2}{|c|}{3 -core } \\
\hline Nodes & DS-RAI \\
\hline 9938 & 844 \\
\hline 10472 & 764 \\
\hline 10512 & 679 \\
\hline 11184 & 818 \\
\hline 11445 & 859 \\
\hline 11555 & 820 \\
\hline 11756 & 479 \\
\hline 12080 & 995 \\
\hline 12151 & 793 \\
\hline 12752 & 858 \\
\hline 13197 & 1376 \\
\hline 13527 & 1278 \\
\hline 13575 & 1039 \\
\hline 13639 & 1280 \\
\hline 13709 & 1448 \\
\hline 13965 & 1085 \\
\hline 14811 & 1219 \\
\hline 14820 & 1175 \\
\hline 14826 & 1234 \\
\hline 14840 & 1249 \\
\hline 15049 & 1093 \\
\hline 15356 & 1006 \\
\hline 16356 & 1605 \\
\hline 16635 & 1611 \\
\hline 16897 & 1278 \\
\hline
\end{tabular}

\begin{tabular}{|c|c|}
\hline \multicolumn{2}{|c|}{3 -core } \\
\hline Nodes & DS-RAI \\
\hline 17045 & 1470 \\
\hline 17101 & 1318 \\
\hline 17323 & 1386 \\
\hline 17387 & 1015 \\
\hline 18043 & 1331 \\
\hline 18377 & 1225 \\
\hline 18561 & 1374 \\
\hline 19558 & 1619 \\
\hline 20314 & 1722 \\
\hline 20700 & 1684 \\
\hline 21109 & 1953 \\
\hline 21424 & 2100 \\
\hline 22717 & 2090 \\
\hline 23624 & 2022 \\
\hline 24298 & 1568 \\
\hline 24400 & 2027 \\
\hline 27555 & 2066 \\
\hline 29600 & 2094 \\
\hline 29913 & 2680 \\
\hline 30601 & 2422 \\
\hline 31438 & 1995 \\
\hline 32119 & 2784 \\
\hline 34878 & 2792 \\
\hline 36107 & 3070 \\
\hline 41137 & 3747 \\
\hline
\end{tabular}

TABLE A.7. Dominating set sizes from the DS-RAI algorithm for the 3-core of MGEO-P samples of the Facebook 100 network. 


\begin{tabular}{|c|c|}
\hline \multicolumn{2}{|c|}{ 4-core } \\
\hline Nodes & DS-RAI \\
\hline 756 & 66 \\
\hline 935 & 99 \\
\hline 1442 & 94 \\
\hline 1484 & 149 \\
\hline 1638 & 132 \\
\hline 2210 & 183 \\
\hline 2233 & 194 \\
\hline 2292 & 185 \\
\hline 2598 & 186 \\
\hline 2607 & 276 \\
\hline 2761 & 229 \\
\hline 2864 & 258 \\
\hline 2935 & 273 \\
\hline 2939 & 222 \\
\hline 3029 & 219 \\
\hline 3043 & 230 \\
\hline 3409 & 281 \\
\hline 3455 & 254 \\
\hline 3547 & 253 \\
\hline 3550 & 320 \\
\hline 3669 & 354 \\
\hline 3789 & 327 \\
\hline 3847 & 313 \\
\hline 4014 & 293 \\
\hline 4060 & 294 \\
\hline
\end{tabular}

\begin{tabular}{|c|c|}
\hline \multicolumn{2}{|c|}{ 4-core } \\
\hline Nodes & DS-RAI \\
\hline 4518 & 336 \\
\hline 5033 & 361 \\
\hline 5132 & 381 \\
\hline 5335 & 386 \\
\hline 6316 & 540 \\
\hline 6392 & 471 \\
\hline 6406 & 499 \\
\hline 6493 & 554 \\
\hline 6537 & 489 \\
\hline 6574 & 525 \\
\hline 6611 & 513 \\
\hline 6733 & 605 \\
\hline 7204 & 668 \\
\hline 7401 & 566 \\
\hline 7620 & 590 \\
\hline 7693 & 570 \\
\hline 7694 & 559 \\
\hline 7702 & 506 \\
\hline 8013 & 549 \\
\hline 8501 & 714 \\
\hline 8523 & 579 \\
\hline 8842 & 863 \\
\hline 8966 & 686 \\
\hline 9325 & 738 \\
\hline 9832 & 630 \\
\hline
\end{tabular}

\begin{tabular}{|c|c|}
\hline \multicolumn{2}{|c|}{4 -core } \\
\hline Nodes & DS-RAI \\
\hline 9901 & 812 \\
\hline 10446 & 742 \\
\hline 10491 & 647 \\
\hline 11164 & 797 \\
\hline 11410 & 832 \\
\hline 11522 & 783 \\
\hline 11748 & 478 \\
\hline 12034 & 941 \\
\hline 12117 & 759 \\
\hline 12721 & 831 \\
\hline 13103 & 1304 \\
\hline 13465 & 1240 \\
\hline 13541 & 1006 \\
\hline 13586 & 1237 \\
\hline 13618 & 1370 \\
\hline 13907 & 1038 \\
\hline 14756 & 1165 \\
\hline 14768 & 1134 \\
\hline 14774 & 1211 \\
\hline 14793 & 1216 \\
\hline 15020 & 1065 \\
\hline 15322 & 969 \\
\hline 16276 & 1535 \\
\hline 16529 & 1530 \\
\hline 16836 & 1228 \\
\hline $4 h e D S$ & $2 A 1$ \\
\hline
\end{tabular}

\begin{tabular}{|c|c|}
\hline \multicolumn{2}{|c|}{ 4-core } \\
\hline Nodes & DS-RAI \\
\hline 16981 & 1428 \\
\hline 17056 & 1279 \\
\hline 17276 & 1353 \\
\hline 17359 & 999 \\
\hline 17994 & 1282 \\
\hline 18336 & 1205 \\
\hline 18517 & 1330 \\
\hline 19486 & 1567 \\
\hline 20238 & 1650 \\
\hline 20620 & 1595 \\
\hline 21025 & 1892 \\
\hline 21317 & 2031 \\
\hline 22602 & 2002 \\
\hline 23528 & 1956 \\
\hline 24247 & 1527 \\
\hline 24310 & 1916 \\
\hline 27465 & 2013 \\
\hline 29519 & 2027 \\
\hline 29798 & 2582 \\
\hline 30504 & 2331 \\
\hline 31371 & 1947 \\
\hline 31985 & 2634 \\
\hline 34788 & 2731 \\
\hline 35994 & 2967 \\
\hline 40947 & 3576 \\
\hline
\end{tabular}

TABLE A.8. Dominating set sizes from the DS-RAI algorithm for the 4-core of MGEO-P samples of the Facebook 100 network. 


\begin{tabular}{|c|c|}
\hline \multicolumn{2}{|c|}{5 -core } \\
\hline Nodes & DS-RAI \\
\hline 751 & 61 \\
\hline 931 & 98 \\
\hline 1437 & 89 \\
\hline 1476 & 140 \\
\hline 1629 & 125 \\
\hline 2203 & 177 \\
\hline 2222 & 181 \\
\hline 2289 & 185 \\
\hline 2581 & 257 \\
\hline 2589 & 179 \\
\hline 2755 & 215 \\
\hline 2850 & 246 \\
\hline 2923 & 210 \\
\hline 2926 & 267 \\
\hline 3015 & 207 \\
\hline 3032 & 225 \\
\hline 3399 & 270 \\
\hline 3448 & 237 \\
\hline 3530 & 302 \\
\hline 3537 & 245 \\
\hline 3649 & 342 \\
\hline 3772 & 306 \\
\hline 3827 & 299 \\
\hline 4005 & 286 \\
\hline 4048 & 281 \\
\hline
\end{tabular}

\begin{tabular}{|c|c|}
\hline \multicolumn{2}{|c|}{5 -core } \\
\hline Nodes & DS-RAI \\
\hline 4503 & 324 \\
\hline 5015 & 346 \\
\hline 5117 & 373 \\
\hline 5323 & 383 \\
\hline 6290 & 519 \\
\hline 6375 & 451 \\
\hline 6382 & 482 \\
\hline 6466 & 539 \\
\hline 6520 & 479 \\
\hline 6552 & 507 \\
\hline 6590 & 497 \\
\hline 6698 & 587 \\
\hline 7163 & 643 \\
\hline 7380 & 552 \\
\hline 7595 & 569 \\
\hline 7668 & 549 \\
\hline 7670 & 534 \\
\hline 7686 & 492 \\
\hline 7999 & 537 \\
\hline 8474 & 688 \\
\hline 8497 & 553 \\
\hline 8787 & 817 \\
\hline 8934 & 666 \\
\hline 9293 & 713 \\
\hline 9805 & 600 \\
\hline
\end{tabular}

\begin{tabular}{|c|c|}
\hline \multicolumn{2}{|c|}{5 -core } \\
\hline Nodes & DS-RAI \\
\hline 9872 & 794 \\
\hline 10426 & 722 \\
\hline 10475 & 631 \\
\hline 11124 & 766 \\
\hline 11378 & 799 \\
\hline 11487 & 760 \\
\hline 11746 & 495 \\
\hline 11992 & 906 \\
\hline 12092 & 742 \\
\hline 12703 & 810 \\
\hline 13029 & 1267 \\
\hline 13384 & 1180 \\
\hline 13496 & 973 \\
\hline 13524 & 1195 \\
\hline 13539 & 1316 \\
\hline 13866 & 999 \\
\hline 14704 & 1131 \\
\hline 14719 & 1161 \\
\hline 14721 & 1152 \\
\hline 14727 & 1090 \\
\hline 14984 & 1022 \\
\hline 15294 & 950 \\
\hline 16180 & 1451 \\
\hline 16443 & 1474 \\
\hline 16785 & 1178 \\
\hline & 2 \\
\hline
\end{tabular}

\begin{tabular}{|c|c|}
\hline \multicolumn{2}{|c|}{5 -core } \\
\hline Nodes & DS-RAI \\
\hline 16907 & 1364 \\
\hline 17006 & 1250 \\
\hline 17230 & 1313 \\
\hline 17337 & 973 \\
\hline 17942 & 1249 \\
\hline 18298 & 1176 \\
\hline 18472 & 1297 \\
\hline 19431 & 1530 \\
\hline 20174 & 1596 \\
\hline 20551 & 1552 \\
\hline 20931 & 1818 \\
\hline 21207 & 1947 \\
\hline 22509 & 1930 \\
\hline 23437 & 1861 \\
\hline 24196 & 1484 \\
\hline 24200 & 1864 \\
\hline 27381 & 1948 \\
\hline 29439 & 1955 \\
\hline 29680 & 2480 \\
\hline 30405 & 2259 \\
\hline 31303 & 1895 \\
\hline 31845 & 2557 \\
\hline 34668 & 2617 \\
\hline 35851 & 2849 \\
\hline 40769 & 3450 \\
\hline
\end{tabular}

TABLE A.9. Dominating set sizes from the DS-RAI algorithm for the 5-core of MGEO-P samples of the Facebook 100 network. 


\begin{tabular}{|c|c|c|c|c|c|c|c|}
\hline \multicolumn{2}{|c|}{0 -core } & \multicolumn{2}{|c|}{ 0-core } & \multicolumn{2}{|c|}{0 -core } & \multicolumn{2}{|c|}{0 -core } \\
\hline Nodes & DS-DC & Nodes & DS-DC & Nodes & DS-DC & Nodes & DS-DC \\
\hline 769 & 49 & 4563 & 216 & 10004 & 524 & 17196 & 814 \\
\hline 962 & 69 & 5075 & 241 & 10521 & 423 & 17212 & 942 \\
\hline 1446 & 62 & 5180 & 254 & 10567 & 411 & 17425 & 788 \\
\hline 1518 & 115 & 5372 & 234 & 11247 & 561 & 17444 & 664 \\
\hline 1659 & 85 & 6386 & 333 & 11509 & 525 & 18163 & 820 \\
\hline 2235 & 104 & 6440 & 291 & 11621 & 505 & 18448 & 717 \\
\hline 2252 & 118 & 6472 & 313 & 11770 & 276 & 18660 & 832 \\
\hline 2314 & 112 & 6591 & 373 & 12155 & 576 & 19700 & 1054 \\
\hline 2613 & 119 & 6596 & 297 & 12193 & 470 & 20467 & 1084 \\
\hline 2682 & 190 & 6637 & 320 & 12803 & 511 & 20871 & 1092 \\
\hline 2790 & 137 & 6682 & 343 & 13377 & 894 & 21325 & 1185 \\
\hline 2920 & 175 & 6833 & 441 & 13653 & 647 & 21679 & 1245 \\
\hline 2970 & 151 & 7324 & 436 & 13686 & 823 & 22937 & 1211 \\
\hline 2970 & 170 & 7460 & 341 & 13746 & 783 & 23842 & 1276 \\
\hline 3068 & 151 & 7694 & 374 & 13882 & 885 & 24389 & 981 \\
\hline 3075 & 150 & 7752 & 360 & 14070 & 719 & 24580 & 1331 \\
\hline 3445 & 165 & 7755 & 318 & 14916 & 714 & 27737 & 1385 \\
\hline 3482 & 160 & 7772 & 362 & 14935 & 791 & 29747 & 1287 \\
\hline 3578 & 171 & 8069 & 337 & 14940 & 821 & 30147 & 1577 \\
\hline 3593 & 199 & 8578 & 360 & 14948 & 820 & 30809 & 1494 \\
\hline 3748 & 257 & 8600 & 445 & 15126 & 647 & 31560 & 1324 \\
\hline 3826 & 186 & 8991 & 585 & 15436 & 656 & 32375 & 1701 \\
\hline 3898 & 209 & 9069 & 520 & 16516 & 959 & 35123 & 1646 \\
\hline 4047 & 181 & 9414 & 457 & 16808 & 979 & 36371 & 1785 \\
\hline 4087 & 176 & 9895 & 404 & 16979 & 751 & 41554 & 2263 \\
\hline
\end{tabular}

TABLE A.10. Dominating set sizes from the DS-DC algorithm for the 0-core of MGEO-P samples of the Facebook 100 network. 


\begin{tabular}{|c|c|c|c|c|c|c|c|}
\hline \multicolumn{2}{|c|}{ 1-core } & \multicolumn{2}{|c|}{ 1-core } & \multicolumn{2}{|c|}{ 1-core } & \multicolumn{2}{|c|}{ 1-core } \\
\hline Nodes & DS-DC & Nodes & DS-DC & Nodes & DS-DC & Nodes & DS-DC \\
\hline 769 & 48 & 4562 & 215 & 10001 & 525 & 17196 & 812 \\
\hline 961 & 68 & 5075 & 240 & 10520 & 424 & 17201 & 932 \\
\hline 1445 & 60 & 5179 & 256 & 10567 & 413 & 17422 & 785 \\
\hline 1517 & 115 & 5372 & 235 & 11247 & 559 & 17443 & 660 \\
\hline 1659 & 85 & 6384 & 332 & 11508 & 521 & 18160 & 818 \\
\hline 2235 & 104 & 6439 & 290 & 11621 & 505 & 18446 & 715 \\
\hline 2252 & 118 & 6471 & 313 & 11770 & 276 & 18658 & 835 \\
\hline 2314 & 112 & 6590 & 372 & 12151 & 571 & 19696 & 1050 \\
\hline 2612 & 118 & 6595 & 300 & 12193 & 472 & 20459 & 1082 \\
\hline 2679 & 188 & 6635 & 319 & 12803 & 509 & 20866 & 1088 \\
\hline 2790 & 137 & 6680 & 341 & 13374 & 890 & 21316 & 1173 \\
\hline 2919 & 174 & 6828 & 442 & 13650 & 647 & 21674 & 1238 \\
\hline 2969 & 168 & 7323 & 439 & 13682 & 819 & 22930 & 1208 \\
\hline 2970 & 153 & 7456 & 336 & 13738 & 773 & 23828 & 1253 \\
\hline 3068 & 151 & 7694 & 376 & 13874 & 875 & 24389 & 980 \\
\hline 3074 & 152 & 7752 & 361 & 14064 & 709 & 24577 & 1328 \\
\hline 3444 & 164 & 7754 & 319 & 14908 & 705 & 27733 & 1382 \\
\hline 3482 & 161 & 7771 & 362 & 14930 & 787 & 29740 & 1280 \\
\hline 3577 & 170 & 8066 & 335 & 14938 & 824 & 30135 & 1568 \\
\hline 3592 & 195 & 8578 & 358 & 14944 & 813 & 30799 & 1480 \\
\hline 3748 & 257 & 8595 & 438 & 15121 & 639 & 31558 & 1327 \\
\hline 3825 & 184 & 8988 & 580 & 15435 & 653 & 32369 & 1695 \\
\hline 3894 & 205 & 9065 & 519 & 16508 & 950 & 35115 & 1630 \\
\hline 4045 & 178 & 9411 & 454 & 16801 & 974 & 36361 & 1772 \\
\hline 4085 & 171 & 9893 & 404 & 16977 & 748 & 41538 & 2252 \\
\hline
\end{tabular}

TABLE A.11. Dominating set sizes from the DS-DC algorithm for the 1-core of MGEO-P samples of the Facebook 100 network. 


\begin{tabular}{|c|c|c|c|c|c|c|c|}
\hline \multicolumn{2}{|c|}{2 -core } & \multicolumn{2}{|c|}{ 2-core } & \multicolumn{2}{|c|}{ 2-core } & \multicolumn{2}{|c|}{ 2-core } \\
\hline Nodes & DS-DC & Nodes & DS-DC & Nodes & DS-DC & Nodes & DS-DC \\
\hline 766 & 49 & 4547 & 203 & 9967 & 501 & 17126 & 882 \\
\hline 957 & 66 & 5063 & 232 & 10504 & 414 & 17152 & 791 \\
\hline 1443 & 61 & 5162 & 247 & 10544 & 398 & 17375 & 763 \\
\hline 1509 & 106 & 5358 & 228 & 11218 & 546 & 17415 & 646 \\
\hline 1653 & 81 & 6366 & 319 & 11479 & 510 & 18102 & 783 \\
\hline 2227 & 100 & 6426 & 283 & 11584 & 489 & 18418 & 696 \\
\hline 2248 & 117 & 6442 & 297 & 11759 & 269 & 18607 & 802 \\
\hline 2306 & 106 & 6555 & 347 & 12117 & 554 & 19620 & 1001 \\
\hline 2607 & 114 & 6575 & 280 & 12170 & 466 & 20376 & 1024 \\
\hline 2662 & 179 & 6618 & 312 & 12778 & 497 & 20792 & 1051 \\
\hline 2783 & 133 & 6661 & 328 & 13280 & 842 & 21223 & 1134 \\
\hline 2902 & 163 & 6793 & 420 & 13608 & 782 & 21551 & 1178 \\
\hline 2960 & 164 & 7276 & 414 & 13617 & 622 & 22839 & 1155 \\
\hline 2961 & 150 & 7433 & 326 & 13697 & 750 & 23723 & 1202 \\
\hline 3054 & 147 & 7667 & 359 & 13799 & 838 & 24334 & 947 \\
\hline 3069 & 147 & 7735 & 353 & 14008 & 684 & 24476 & 1270 \\
\hline 3433 & 160 & 7738 & 310 & 14870 & 748 & 27648 & 1335 \\
\hline 3472 & 149 & 7745 & 349 & 14872 & 689 & 29678 & 1246 \\
\hline 3563 & 162 & 8047 & 321 & 14885 & 797 & 30020 & 1501 \\
\hline 3578 & 188 & 8554 & 411 & 14886 & 785 & 30707 & 1421 \\
\hline 3719 & 241 & 8559 & 353 & 15086 & 615 & 31493 & 1288 \\
\hline 3813 & 180 & 8947 & 555 & 15394 & 640 & 32256 & 1626 \\
\hline 3877 & 197 & 9035 & 507 & 16428 & 897 & 34989 & 1579 \\
\hline 4032 & 171 & 9385 & 441 & 16709 & 936 & 36233 & 1714 \\
\hline 4080 & 172 & 9868 & 388 & 16938 & 727 & 41344 & 2139 \\
\hline
\end{tabular}

TABLE A.12. Dominating set sizes from the DS-DC algorithm for the 2-core of MGEO-P samples of the Facebook 100 network. 


\begin{tabular}{|c|c|}
\hline \multicolumn{2}{|c|}{3 -core } \\
\hline Nodes & DS-DC \\
\hline 761 & 45 \\
\hline 947 & 65 \\
\hline 1442 & 60 \\
\hline 1494 & 95 \\
\hline 1649 & 80 \\
\hline 2222 & 97 \\
\hline 2239 & 111 \\
\hline 2298 & 103 \\
\hline 2605 & 113 \\
\hline 2632 & 158 \\
\hline 2771 & 126 \\
\hline 2887 & 155 \\
\hline 2945 & 159 \\
\hline 2948 & 144 \\
\hline 3039 & 140 \\
\hline 3057 & 139 \\
\hline 3419 & 155 \\
\hline 3462 & 145 \\
\hline 3552 & 154 \\
\hline 3563 & 180 \\
\hline 3695 & 227 \\
\hline 3806 & 178 \\
\hline 3856 & 191 \\
\hline 4021 & 161 \\
\hline 4071 & 170 \\
\hline & $\mathrm{A}$ \\
\hline 35 \\
\hline
\end{tabular}

\begin{tabular}{|c|c|}
\hline \multicolumn{2}{|c|}{3 -core } \\
\hline Nodes & DS-DC \\
\hline 4537 & 203 \\
\hline 5047 & 225 \\
\hline 5148 & 235 \\
\hline 5345 & 219 \\
\hline 6337 & 306 \\
\hline 6412 & 276 \\
\hline 6424 & 283 \\
\hline 6518 & 325 \\
\hline 6552 & 274 \\
\hline 6594 & 300 \\
\hline 6634 & 312 \\
\hline 6764 & 402 \\
\hline 7249 & 405 \\
\hline 7420 & 325 \\
\hline 7641 & 345 \\
\hline 7715 & 346 \\
\hline 7722 & 301 \\
\hline 7724 & 334 \\
\hline 8030 & 314 \\
\hline 8527 & 397 \\
\hline 8545 & 345 \\
\hline 8891 & 528 \\
\hline 8999 & 486 \\
\hline 9358 & 423 \\
\hline 9850 & 377 \\
\hline & 1 \\
1 & 1 \\
1 & 1 \\
1 & 1 \\
1 & 1 \\
\hline
\end{tabular}

\begin{tabular}{|c|c|}
\hline \multicolumn{2}{|c|}{3 -core } \\
\hline Nodes & DS-DC \\
\hline 9938 & 493 \\
\hline 10472 & 402 \\
\hline 10512 & 384 \\
\hline 11184 & 531 \\
\hline 11445 & 495 \\
\hline 11555 & 472 \\
\hline 11756 & 269 \\
\hline 12080 & 541 \\
\hline 12151 & 453 \\
\hline 12752 & 481 \\
\hline 13197 & 812 \\
\hline 13527 & 740 \\
\hline 13575 & 610 \\
\hline 13639 & 726 \\
\hline 13709 & 786 \\
\hline 13965 & 660 \\
\hline 14811 & 728 \\
\hline 14820 & 668 \\
\hline 14826 & 772 \\
\hline 14840 & 763 \\
\hline 15049 & 596 \\
\hline 15356 & 616 \\
\hline 16356 & 864 \\
\hline 16635 & 896 \\
\hline 16897 & 717 \\
\hline $149 D S-D C$ \\
\hline
\end{tabular}

\begin{tabular}{|c|c|}
\hline \multicolumn{2}{|c|}{3 -core } \\
\hline Nodes & DS-DC \\
\hline 17045 & 849 \\
\hline 17101 & 768 \\
\hline 17323 & 730 \\
\hline 17387 & 633 \\
\hline 18043 & 745 \\
\hline 18377 & 676 \\
\hline 18561 & 781 \\
\hline 19558 & 975 \\
\hline 20314 & 988 \\
\hline 20700 & 1005 \\
\hline 21109 & 1074 \\
\hline 21424 & 1113 \\
\hline 22717 & 1090 \\
\hline 23624 & 1151 \\
\hline 24298 & 938 \\
\hline 24400 & 1231 \\
\hline 27555 & 1281 \\
\hline 29600 & 1208 \\
\hline 29913 & 1442 \\
\hline 30601 & 1369 \\
\hline 31438 & 1260 \\
\hline 32119 & 1566 \\
\hline 34878 & 1516 \\
\hline 36107 & 1646 \\
\hline 41137 & 2045 \\
\hline
\end{tabular}

TABLE A.13. Dominating set sizes from the DS-DC algorithm for the 3-core of MGEO-P samples of the Facebook 100 network. 


\begin{tabular}{|c|c|c|c|c|c|c|c|}
\hline \multicolumn{2}{|c|}{ 4-core } & \multicolumn{2}{|c|}{ 4-core } & \multicolumn{2}{|c|}{ 4-core } & \multicolumn{2}{|c|}{ 4-core } \\
\hline Nodes & DS-DC & Nodes & DS-DC & Nodes & DS-DC & Nodes & DS-DC \\
\hline 756 & 50 & 4518 & 199 & 9901 & 471 & 17056 & 743 \\
\hline 935 & 58 & 5033 & 215 & 11164 & 525 & 16981 & 819 \\
\hline 1484 & 90 & 5132 & 231 & 10491 & 379 & 17276 & 712 \\
\hline 1442 & 60 & 5335 & 216 & 11410 & 471 & 17359 & 621 \\
\hline 1638 & 74 & 6493 & 311 & 10446 & 388 & 17994 & 718 \\
\hline 2607 & 147 & 6316 & 300 & 11748 & 263 & 18517 & 751 \\
\hline 2233 & 106 & 6392 & 262 & 12117 & 443 & 18336 & 662 \\
\hline 2210 & 94 & 6574 & 294 & 11522 & 462 & 19486 & 937 \\
\hline 2292 & 102 & 6406 & 277 & 12034 & 517 & 20238 & 959 \\
\hline 2598 & 109 & 6537 & 266 & 12721 & 472 & 21025 & 1038 \\
\hline 2864 & 144 & 6733 & 391 & 13103 & 762 & 20620 & 968 \\
\hline 2939 & 137 & 7204 & 381 & 13465 & 708 & 21317 & 1056 \\
\hline 2935 & 152 & 6611 & 306 & 13541 & 586 & 22602 & 1044 \\
\hline 2761 & 121 & 7694 & 350 & 13618 & 751 & 23528 & 1103 \\
\hline 3669 & 220 & 7620 & 334 & 13586 & 703 & 24247 & 912 \\
\hline 3029 & 135 & 7401 & 311 & 13907 & 629 & 24310 & 1192 \\
\hline 3043 & 130 & 7693 & 321 & 14774 & 745 & 27465 & 1242 \\
\hline 3550 & 168 & 7702 & 290 & 14756 & 702 & 29798 & 1411 \\
\hline 3409 & 152 & 8013 & 310 & 14768 & 647 & 29519 & 1175 \\
\hline 3547 & 153 & 8523 & 337 & 14793 & 740 & 31371 & 1232 \\
\hline 3455 & 143 & 8842 & 510 & 15020 & 592 & 30504 & 1343 \\
\hline 3847 & 188 & 8966 & 473 & 16529 & 853 & 31985 & 1519 \\
\hline 3789 & 171 & 8501 & 381 & 16276 & 837 & 34788 & 1476 \\
\hline 4060 & 166 & 9325 & 404 & 15322 & 595 & 35994 & 1611 \\
\hline 4014 & 159 & 9832 & 365 & 16836 & 693 & 40947 & 1966 \\
\hline
\end{tabular}

TABLE A.14. Dominating set sizes from the DS-DC algorithm for the 4-core of MGEO-P samples of the Facebook 100 network. 


\begin{tabular}{|c|c|c|c|c|c|c|c|}
\hline \multicolumn{2}{|c|}{ 5-core } & \multicolumn{2}{|c|}{ 5-core } & \multicolumn{2}{|c|}{ 5-core } & \multicolumn{2}{|c|}{ 5-core } \\
\hline Nodes & DS-DC & Nodes & DS-DC & Nodes & DS-DC & Nodes & DS-DC \\
\hline 751 & 47 & 4503 & 196 & 9872 & 457 & 16907 & 786 \\
\hline 931 & 57 & 5015 & 209 & 10426 & 378 & 17006 & 721 \\
\hline 1437 & 56 & 5117 & 224 & 10475 & 374 & 17230 & 694 \\
\hline 1476 & 89 & 5323 & 208 & 11124 & 516 & 17337 & 605 \\
\hline 1629 & 67 & 6290 & 289 & 11378 & 456 & 17942 & 698 \\
\hline 2203 & 91 & 6375 & 256 & 11487 & 439 & 18298 & 646 \\
\hline 2222 & 105 & 6382 & 265 & 11746 & 262 & 18472 & 741 \\
\hline 2289 & 99 & 6466 & 304 & 11992 & 499 & 19431 & 918 \\
\hline 2581 & 137 & 6520 & 258 & 12092 & 428 & 20174 & 941 \\
\hline 2589 & 106 & 6552 & 290 & 12703 & 464 & 20551 & 944 \\
\hline 2755 & 116 & 6590 & 298 & 13029 & 741 & 20931 & 1013 \\
\hline 2850 & 140 & 6698 & 379 & 13384 & 673 & 21207 & 1019 \\
\hline 2923 & 133 & 7163 & 365 & 13496 & 575 & 22509 & 1012 \\
\hline 2926 & 150 & 7380 & 302 & 13524 & 690 & 23437 & 1068 \\
\hline 3015 & 128 & 7595 & 331 & 13539 & 726 & 24196 & 897 \\
\hline 3032 & 124 & 7668 & 310 & 13866 & 617 & 24200 & 1148 \\
\hline 3399 & 146 & 7670 & 337 & 14704 & 684 & 27381 & 1213 \\
\hline 3448 & 140 & 7686 & 284 & 14719 & 721 & 29439 & 1141 \\
\hline 3530 & 157 & 7999 & 301 & 14721 & 717 & 29680 & 1357 \\
\hline 3537 & 144 & 8474 & 373 & 14727 & 621 & 30405 & 1304 \\
\hline 3649 & 205 & 8497 & 324 & 14984 & 572 & 31303 & 1199 \\
\hline 3772 & 168 & 8787 & 491 & 15294 & 584 & 31845 & 1474 \\
\hline 3827 & 185 & 8934 & 461 & 16180 & 812 & 34668 & 1415 \\
\hline 4005 & 158 & 9293 & 394 & 16443 & 820 & 35851 & 1550 \\
\hline 4048 & 160 & 9805 & 347 & 16785 & 665 & 40769 & 1892 \\
\hline
\end{tabular}

TABLE A.15. Dominating set sizes from the DS-DC algorithm for the 5-core of MGEO-P samples of the Facebook 100 network. 


\begin{tabular}{|c|c|c|c|c|c|}
\hline Dataset Name & Nodes & Edges & Average Degree & Maximum Degree & Density \\
\hline Caltech36 & 769 & 16656 & 43 & 248 & 0.056 \\
\hline Reed98 & 962 & 18812 & 39 & 313 & 0.041 \\
\hline Haverford76 & 1446 & 59589 & 82 & 375 & 0.057 \\
\hline Simmons81 & 1518 & 32988 & 43 & 300 & 0.029 \\
\hline Swarthmore42 & 1659 & 61050 & 74 & 577 & 0.044 \\
\hline Amherst41 & 2235 & 90954 & 81 & 467 & 0.036 \\
\hline Bowdoin47 & 2252 & 84387 & 75 & 670 & 0.033 \\
\hline Hamilton46 & 2314 & 96394 & 83 & 602 & 0.036 \\
\hline Trinity100 & 2613 & 111996 & 86 & 404 & 0.033 \\
\hline USFCA72 & 2682 & 65252 & 49 & 405 & 0.018 \\
\hline Williams40 & 2790 & 112986 & 81 & 610 & 0.029 \\
\hline Oberlin44 & 2920 & 89912 & 62 & 478 & 0.021 \\
\hline Smith60 & 2970 & 97133 & 65 & 349 & 0.022 \\
\hline Wellesley22 & 2970 & 94899 & 64 & 746 & 0.022 \\
\hline Vassar85 & 3068 & 119161 & 78 & 482 & 0.025 \\
\hline Middlebury45 & 3075 & 124610 & 81 & 473 & 0.026 \\
\hline Pepperdine86 & 3445 & 152007 & 88 & 674 & 0.026 \\
\hline Colgate88 & 3482 & 155043 & 89 & 773 & 0.026 \\
\hline Santa74 & 3578 & 151747 & 85 & 1129 & 0.024 \\
\hline Wesleyan43 & 3593 & 138035 & 77 & 549 & 0.021 \\
\hline Mich67 & 3748 & 81903 & 44 & 419 & 0.012 \\
\hline Bucknell39 & 3826 & 158864 & 83 & 506 & 0.022 \\
\hline Brandeis99 & 3898 & 137567 & 71 & 1972 & 0.018 \\
\hline Howard90 & 4047 & 204850 & 101 & 1215 & 0.025 \\
\hline Rice31 & 4087 & 184828 & 90 & 581 & 0.022 \\
\hline Rochester38 & 4563 & 161404 & 71 & 1224 & 0.016 \\
\hline Lehigh96 & 5075 & 198347 & 78 & 973 & 0.015 \\
\hline Johns-Hopkins55 & 5180 & 186586 & 72 & 886 & 0.014 \\
\hline Wake73 & 5372 & 279191 & 104 & 1341 & 0.019 \\
\hline American75 & 6386 & 217662 & 68 & 930 & 0.011 \\
\hline MIT8 & 6440 & 251252 & 78 & 708 & 0.012 \\
\hline William77 & 6472 & 266378 & 82 & 1124 & 0.013 \\
\hline UChicago30 & 6591 & 208103 & 63 & 1624 & 0.010 \\
\hline Princeton12 & 6596 & 293320 & 89 & 628 & 0.013 \\
\hline Carnegie49 & 6637 & 249967 & 75 & 840 & 0.011 \\
\hline Tufts18 & 6682 & 249728 & 75 & 827 & 0.011 \\
\hline UC64 & 6833 & 155332 & 45 & 660 & 0.007 \\
\hline Vermont70 & 7324 & 191221 & 52 & 864 & 0.007 \\
\hline Emory27 & 7460 & 330014 & 88 & 1095 & 0.012 \\
\hline TAs6e A.16. & Ge & $5 a c b 00$ & \\
\hline
\end{tabular}

TABlE A.16. General graph properties computed for Facebook 100.

\section{A.2. Facebook 100 Data Tables}




\begin{tabular}{|c|c|c|c|c|c|}
\hline Dataset Name & Nodes & Edges & Average Degree & Maximum Degree & Density \\
\hline Dartmouth6 & 7694 & 304076 & 79 & 948 & 0.010 \\
\hline Tulane29 & 7752 & 283918 & 73 & 1188 & 0.009 \\
\hline WashU32 & 7755 & 367541 & 95 & 1794 & 0.012 \\
\hline Villanova62 & 7772 & 314989 & 81 & 1183 & 0.010 \\
\hline Vanderbilt48 & 8069 & 427832 & 106 & 2041 & 0.013 \\
\hline Yale4 & 8578 & 405450 & 95 & 2517 & 0.011 \\
\hline Brown11 & 8600 & 384526 & 89 & 1075 & 0.010 \\
\hline UCSC68 & 8991 & 224584 & 50 & 454 & 0.006 \\
\hline Maine59 & 9069 & 243247 & 54 & 1011 & 0.006 \\
\hline Georgetown15 & 9414 & 425638 & 90 & 1235 & 0.009 \\
\hline Duke14 & 9895 & 506442 & 102 & 1887 & 0.010 \\
\hline Bingham82 & 10004 & 362894 & 73 & 553 & 0.007 \\
\hline Mississippi66 & 10521 & 610911 & 116 & 1691 & 0.011 \\
\hline Northwestern25 & 10567 & 488337 & 92 & 2105 & 0.009 \\
\hline Cal65 & 11247 & 351358 & 62 & 415 & 0.006 \\
\hline BC17 & 11509 & 486967 & 85 & 1377 & 0.007 \\
\hline Stanford3 & 11621 & 568330 & 98 & 1172 & 0.008 \\
\hline Columbia2 & 11770 & 444333 & 76 & 3375 & 0.006 \\
\hline Notre-Dame57 & 12155 & 541339 & 89 & 1344 & 0.007 \\
\hline GWU54 & 12193 & 469528 & 77 & 2002 & 0.006 \\
\hline Baylor93 & 12803 & 679817 & 106 & 2109 & 0.008 \\
\hline USF51 & 13377 & 321214 & 48 & 897 & 0.004 \\
\hline Syracuse56 & 13653 & 543982 & 80 & 1340 & 0.006 \\
\hline Temple83 & 13686 & 360795 & 53 & 1394 & 0.004 \\
\hline UC61 & 13746 & 442174 & 64 & 687 & 0.005 \\
\hline Northeastern19 & 13882 & 381934 & 55 & 968 & 0.004 \\
\hline JMU79 & 14070 & 485564 & 69 & 3274 & 0.005 \\
\hline UPenn7 & 14916 & 686501 & 92 & 1602 & 0.006 \\
\hline UCSB37 & 14935 & 482224 & 65 & 810 & 0.004 \\
\hline UCF52 & 14940 & 428989 & 57 & 4765 & 0.004 \\
\hline UCSD34 & 14948 & 443221 & 59 & 2165 & 0.004 \\
\hline Harvard1 & 15126 & 824617 & 109 & 1183 & 0.007 \\
\hline MU78 & 15436 & 649449 & 84 & 653 & 0.005 \\
\hline UMass92 & 16516 & 519385 & 63 & 3684 & 0.004 \\
\hline UC33 & 16808 & 522147 & 62 & 1415 & 0.004 \\
\hline Tennessee95 & 16979 & 770659 & 91 & 4943 & 0.005 \\
\hline UVA16 & 17196 & 789321 & 92 & 3182 & 0.005 \\
\hline UConn91 & 17212 & 604870 & 70 & 1709 & 0.004 \\
\hline Oklahoma97 & 17425 & 892528 & 102 & 2568 & 0.006 \\
\hline USC35 & 17444 & 801853 & 92 & 4459 & 0.005 \\
\hline
\end{tabular}

TABLE A.17. General graph properties computed for Facebook 100 (continued). 


\begin{tabular}{|c|c|c|c|c|c|}
\hline Dataset Name & Nodes & Edges & Average Degree & Maximum Degree & Density \\
\hline UNC28 & 18163 & 766800 & 84 & 3795 & 0.005 \\
\hline Auburn71 & 18448 & 973918 & 106 & 5160 & 0.006 \\
\hline Cornell5 & 18660 & 790777 & 85 & 3156 & 0.005 \\
\hline BU10 & 19700 & 637528 & 65 & 1819 & 0.003 \\
\hline UCLA26 & 20467 & 747613 & 73 & 1180 & 0.004 \\
\hline Maryland58 & 20871 & 744862 & 71 & 3784 & 0.003 \\
\hline Virginia63 & 21325 & 698178 & 65 & 7206 & 0.003 \\
\hline NYU9 & 21679 & 715715 & 66 & 2315 & 0.003 \\
\hline Berkeley13 & 22937 & 852444 & 74 & 3434 & 0.003 \\
\hline Wisconsin87 & 23842 & 835952 & 70 & 3484 & 0.003 \\
\hline UGA50 & 24389 & 1174057 & 96 & 2926 & 0.004 \\
\hline Rutgers89 & 24580 & 784602 & 64 & 1642 & 0.003 \\
\hline FSU53 & 27737 & 1034802 & 75 & 2555 & 0.003 \\
\hline Indiana69 & 29747 & 1305765 & 88 & 1358 & 0.003 \\
\hline Michigan23 & 30147 & 1176516 & 78 & 2031 & 0.003 \\
\hline UIllinois20 & 30809 & 1264428 & 82 & 4632 & 0.003 \\
\hline Texas80 & 31560 & 1219650 & 77 & 1796 & 0.002 \\
\hline MSU24 & 32375 & 1118774 & 69 & 5267 & 0.002 \\
\hline UF21 & 35123 & 1465660 & 83 & 8246 & 0.002 \\
\hline Texas84 & 36371 & 1590655 & 87 & 6312 & 0.002 \\
\hline Penn94 & 41554 & 1362229 & 66 & 4410 & 0.002 \\
\hline
\end{tabular}

TABLE A.18. General graph properties computed for Facebook 100 (continued). 


\begin{tabular}{|c|c|}
\hline \multicolumn{2}{|c|}{ 1-core } \\
\hline Nodes & DS-RAI \\
\hline 769 & 131 \\
\hline 962 & 150 \\
\hline 1446 & 143 \\
\hline 1518 & 221 \\
\hline 1659 & 187 \\
\hline 2235 & 240 \\
\hline 2252 & 254 \\
\hline 2314 & 242 \\
\hline 2613 & 267 \\
\hline 2682 & 458 \\
\hline 2790 & 285 \\
\hline 2920 & 422 \\
\hline 2970 & 378 \\
\hline 2970 & 348 \\
\hline 3068 & 321 \\
\hline 3075 & 394 \\
\hline 3445 & 466 \\
\hline 3482 & 348 \\
\hline 3578 & 427 \\
\hline 3593 & 386 \\
\hline 3748 & 650 \\
\hline 3826 & 368 \\
\hline 3898 & 475 \\
\hline 4047 & 534 \\
\hline 4087 & 494 \\
\hline
\end{tabular}

\begin{tabular}{|c|c|}
\hline \multicolumn{2}{|c|}{ 1-core } \\
\hline Nodes & DS-RAI \\
\hline 4563 & 610 \\
\hline 5075 & 579 \\
\hline 5180 & 842 \\
\hline 5372 & 573 \\
\hline 6386 & 989 \\
\hline 6440 & 1118 \\
\hline 6472 & 774 \\
\hline 6591 & 1144 \\
\hline 6596 & 904 \\
\hline 6637 & 1056 \\
\hline 6682 & 951 \\
\hline 6833 & 1334 \\
\hline 7324 & 1182 \\
\hline 7460 & 1086 \\
\hline 7694 & 1364 \\
\hline 7752 & 1186 \\
\hline 7755 & 974 \\
\hline 7772 & 1051 \\
\hline 8069 & 979 \\
\hline 8578 & 1218 \\
\hline 8600 & 1186 \\
\hline 8991 & 1468 \\
\hline 9069 & 1508 \\
\hline 9414 & 1227 \\
\hline 9895 & 1369 \\
\hline $7+179$ & 1865 \\
\hline
\end{tabular}

\begin{tabular}{|c|c|}
\hline \multicolumn{2}{|c|}{1 -core } \\
\hline Nodes & DS-RAI \\
\hline 10004 & 1405 \\
\hline 10521 & 1392 \\
\hline 10567 & 1503 \\
\hline 11247 & 1672 \\
\hline 11509 & 1524 \\
\hline 11621 & 1967 \\
\hline 11770 & 1972 \\
\hline 12155 & 1495 \\
\hline 12193 & 1816 \\
\hline 12803 & 1598 \\
\hline 13377 & 2670 \\
\hline 13653 & 1801 \\
\hline 13686 & 2658 \\
\hline 13746 & 2367 \\
\hline 13882 & 2263 \\
\hline 14070 & 1861 \\
\hline 14916 & 2246 \\
\hline 14935 & 2388 \\
\hline 14940 & 2534 \\
\hline 14948 & 2545 \\
\hline 15126 & 2513 \\
\hline 15436 & 1926 \\
\hline 16516 & 2500 \\
\hline 16808 & 2757 \\
\hline 16979 & 2370 \\
\hline $1 h e D S-R A 1$ \\
\hline
\end{tabular}

\begin{tabular}{|c|c|}
\hline \multicolumn{2}{|c|}{1 -core } \\
\hline Nodes & DS-RAI \\
\hline 17196 & 2442 \\
\hline 17212 & 2505 \\
\hline 17425 & 2549 \\
\hline 17444 & 2679 \\
\hline 18163 & 2660 \\
\hline 18448 & 2310 \\
\hline 18660 & 2628 \\
\hline 19700 & 2984 \\
\hline 20467 & 3437 \\
\hline 20871 & 3183 \\
\hline 21325 & 3275 \\
\hline 21679 & 3631 \\
\hline 22937 & 3739 \\
\hline 23842 & 3376 \\
\hline 24389 & 3017 \\
\hline 24580 & 4077 \\
\hline 27737 & 4215 \\
\hline 29747 & 3972 \\
\hline 30147 & 4719 \\
\hline 30809 & 4103 \\
\hline 31560 & 4678 \\
\hline 32375 & 4785 \\
\hline 35123 & 4919 \\
\hline 36371 & 5231 \\
\hline 41554 & 6755 \\
\hline
\end{tabular}

TABLE A.19. Dominating set sizes from the DS-RAI algorithm for the 1-core of Facebook 100 graphs. 


\begin{tabular}{|c|c|}
\hline \multicolumn{2}{|c|}{2 -core } \\
\hline Nodes & DS-RAI \\
\hline 734 & 102 \\
\hline 927 & 127 \\
\hline 1428 & 126 \\
\hline 1468 & 185 \\
\hline 1644 & 176 \\
\hline 2199 & 210 \\
\hline 2214 & 227 \\
\hline 2281 & 216 \\
\hline 2575 & 232 \\
\hline 2589 & 379 \\
\hline 2743 & 245 \\
\hline 2857 & 349 \\
\hline 2910 & 294 \\
\hline 2915 & 334 \\
\hline 3013 & 345 \\
\hline 3034 & 298 \\
\hline 3342 & 381 \\
\hline 3439 & 310 \\
\hline 3522 & 381 \\
\hline 3526 & 333 \\
\hline 3629 & 552 \\
\hline 3794 & 398 \\
\hline 3795 & 337 \\
\hline 3956 & 458 \\
\hline 4006 & 427 \\
\hline
\end{tabular}

\begin{tabular}{|c|c|}
\hline \multicolumn{2}{|c|}{2 -core } \\
\hline Nodes & DS-RAI \\
\hline 4484 & 535 \\
\hline 4979 & 678 \\
\hline 5001 & 506 \\
\hline 5291 & 504 \\
\hline 6129 & 873 \\
\hline 6194 & 840 \\
\hline 6318 & 938 \\
\hline 6371 & 686 \\
\hline 6392 & 737 \\
\hline 6426 & 884 \\
\hline 6490 & 796 \\
\hline 6514 & 1087 \\
\hline 7104 & 996 \\
\hline 7260 & 912 \\
\hline 7370 & 1083 \\
\hline 7562 & 1041 \\
\hline 7570 & 889 \\
\hline 7578 & 826 \\
\hline 7903 & 854 \\
\hline 8292 & 996 \\
\hline 8380 & 1010 \\
\hline 8716 & 1240 \\
\hline 8813 & 1275 \\
\hline 9141 & 1019 \\
\hline 9621 & 1139 \\
\hline
\end{tabular}

\begin{tabular}{|c|c|}
\hline \multicolumn{2}{|c|}{2 -core } \\
\hline Nodes & DS-RAI \\
\hline 9800 & 1231 \\
\hline 10270 & 1265 \\
\hline 10356 & 1245 \\
\hline 10996 & 1456 \\
\hline 11087 & 1554 \\
\hline 11185 & 1561 \\
\hline 11253 & 1311 \\
\hline 11842 & 1520 \\
\hline 11951 & 1318 \\
\hline 12636 & 1444 \\
\hline 12892 & 2252 \\
\hline 13171 & 2255 \\
\hline 13277 & 1983 \\
\hline 13376 & 1564 \\
\hline 13511 & 1968 \\
\hline 13868 & 1665 \\
\hline 14398 & 1816 \\
\hline 14443 & 1965 \\
\hline 14503 & 2177 \\
\hline 14533 & 2054 \\
\hline 14600 & 2243 \\
\hline 15199 & 1730 \\
\hline 16168 & 2197 \\
\hline 16342 & 2375 \\
\hline 16638 & 2080 \\
\hline
\end{tabular}

\begin{tabular}{|c|c|}
\hline \multicolumn{2}{|c|}{2 -core } \\
\hline Nodes & DS-RAI \\
\hline 16801 & 2084 \\
\hline 16832 & 2174 \\
\hline 16942 & 2259 \\
\hline 17032 & 2232 \\
\hline 17799 & 2364 \\
\hline 18138 & 2216 \\
\hline 18187 & 2068 \\
\hline 19176 & 2581 \\
\hline 19818 & 2900 \\
\hline 20311 & 2759 \\
\hline 20789 & 2804 \\
\hline 20892 & 2974 \\
\hline 22243 & 3164 \\
\hline 23326 & 2955 \\
\hline 23902 & 3494 \\
\hline 23966 & 2644 \\
\hline 27175 & 3707 \\
\hline 29184 & 3483 \\
\hline 29288 & 3998 \\
\hline 30203 & 3592 \\
\hline 30898 & 4116 \\
\hline 31640 & 4155 \\
\hline 34514 & 4361 \\
\hline 35694 & 4614 \\
\hline 40458 & 5782 \\
\hline
\end{tabular}

TABLE A.20. Dominating set sizes from the DS-RAI algorithm for the 2-core of Facebook 100 graphs. 


\begin{tabular}{|c|c|}
\hline \multicolumn{2}{|c|}{3 -core } \\
\hline Nodes & DS-RAI \\
\hline 699 & 83 \\
\hline 906 & 114 \\
\hline 1414 & 120 \\
\hline 1438 & 165 \\
\hline 1630 & 163 \\
\hline 2176 & 188 \\
\hline 2192 & 211 \\
\hline 2264 & 205 \\
\hline 2526 & 336 \\
\hline 2548 & 211 \\
\hline 2719 & 221 \\
\hline 2817 & 337 \\
\hline 2863 & 306 \\
\hline 2880 & 266 \\
\hline 2971 & 308 \\
\hline 3005 & 276 \\
\hline 3276 & 339 \\
\hline 3424 & 298 \\
\hline 3484 & 304 \\
\hline 3491 & 355 \\
\hline 3544 & 496 \\
\hline 3741 & 364 \\
\hline 3774 & 323 \\
\hline 3902 & 414 \\
\hline 3954 & 395 \\
\hline
\end{tabular}

\begin{tabular}{|c|c|}
\hline \multicolumn{2}{|c|}{3 -core } \\
\hline Nodes & DS-RAI \\
\hline 4415 & 490 \\
\hline 4840 & 587 \\
\hline 4945 & 475 \\
\hline 5242 & 467 \\
\hline 5953 & 761 \\
\hline 6061 & 746 \\
\hline 6153 & 824 \\
\hline 6266 & 924 \\
\hline 6275 & 665 \\
\hline 6281 & 784 \\
\hline 6308 & 646 \\
\hline 6379 & 725 \\
\hline 6964 & 898 \\
\hline 7121 & 811 \\
\hline 7180 & 941 \\
\hline 7403 & 922 \\
\hline 7444 & 724 \\
\hline 7457 & 808 \\
\hline 7802 & 778 \\
\hline 8114 & 871 \\
\hline 8250 & 918 \\
\hline 8517 & 1122 \\
\hline 8642 & 1157 \\
\hline 8984 & 918 \\
\hline 9459 & 1027 \\
\hline
\end{tabular}

\begin{tabular}{|c|c|}
\hline \multicolumn{2}{|c|}{3 -core } \\
\hline Nodes & DS-RAI \\
\hline 9665 & 1111 \\
\hline 10082 & 1128 \\
\hline 10243 & 1144 \\
\hline 10767 & 1332 \\
\hline 10816 & 1345 \\
\hline 10852 & 1355 \\
\hline 11108 & 1215 \\
\hline 11624 & 1362 \\
\hline 11833 & 1224 \\
\hline 12495 & 1995 \\
\hline 12502 & 1345 \\
\hline 12777 & 1980 \\
\hline 12962 & 1743 \\
\hline 13174 & 1424 \\
\hline 13242 & 1777 \\
\hline 13734 & 1572 \\
\hline 14002 & 1649 \\
\hline 14105 & 1635 \\
\hline 14209 & 1974 \\
\hline 14272 & 1855 \\
\hline 14299 & 2036 \\
\hline 15031 & 1609 \\
\hline 15925 & 2005 \\
\hline 16008 & 2110 \\
\hline 16408 & 1897 \\
\hline $140 \mathrm{DS}$ & $\mathrm{RA}$ \\
\hline
\end{tabular}

\begin{tabular}{|c|c|}
\hline \multicolumn{2}{|c|}{3 -core } \\
\hline Nodes & DS-RAI \\
\hline 39703 & 5236 \\
\hline 16540 & 1910 \\
\hline 16587 & 1986 \\
\hline 16637 & 2013 \\
\hline 16785 & 2040 \\
\hline 17536 & 2164 \\
\hline 17811 & 1988 \\
\hline 18022 & 1939 \\
\hline 18808 & 2317 \\
\hline 19342 & 2566 \\
\hline 19998 & 2525 \\
\hline 20405 & 2652 \\
\hline 20432 & 2532 \\
\hline 21800 & 2841 \\
\hline 22964 & 2693 \\
\hline 23440 & 3158 \\
\hline 23718 & 2461 \\
\hline 26771 & 3344 \\
\hline 28750 & 3607 \\
\hline 28796 & 3197 \\
\hline 29781 & 3259 \\
\hline 30457 & 3785 \\
\hline 31157 & 3773 \\
\hline 34080 & 4019 \\
\hline 35240 & 4239 \\
\hline
\end{tabular}

TABLE A.21. Dominating set sizes from the DS-RAI algorithm for the 3-core of Facebook 100 graphs. 


\begin{tabular}{|c|c|}
\hline \multicolumn{2}{|c|}{ 4-core } \\
\hline Nodes & DS-RAI \\
\hline 684 & 75 \\
\hline 891 & 103 \\
\hline 1408 & 113 \\
\hline 1411 & 150 \\
\hline 1614 & 155 \\
\hline 2153 & 179 \\
\hline 2170 & 199 \\
\hline 2241 & 187 \\
\hline 2470 & 304 \\
\hline 2530 & 200 \\
\hline 2701 & 214 \\
\hline 2772 & 312 \\
\hline 2831 & 296 \\
\hline 2851 & 243 \\
\hline 2936 & 285 \\
\hline 2990 & 267 \\
\hline 3224 & 302 \\
\hline 3404 & 287 \\
\hline 3455 & 334 \\
\hline 3460 & 291 \\
\hline 3462 & 459 \\
\hline 3692 & 336 \\
\hline 3744 & 300 \\
\hline 3858 & 386 \\
\hline 3905 & 366 \\
\hline
\end{tabular}

\begin{tabular}{|c|c|}
\hline \multicolumn{2}{|c|}{ 4-core } \\
\hline Nodes & DS-RAI \\
\hline 4364 & 454 \\
\hline 4765 & 539 \\
\hline 4895 & 439 \\
\hline 5203 & 444 \\
\hline 5842 & 689 \\
\hline 5954 & 677 \\
\hline 5994 & 740 \\
\hline 6070 & 823 \\
\hline 6181 & 604 \\
\hline 6189 & 718 \\
\hline 6235 & 603 \\
\hline 6291 & 674 \\
\hline 6840 & 812 \\
\hline 7020 & 742 \\
\hline 7039 & 849 \\
\hline 7286 & 864 \\
\hline 7367 & 754 \\
\hline 7372 & 685 \\
\hline 7701 & 719 \\
\hline 7970 & 783 \\
\hline 8131 & 840 \\
\hline 8351 & 1032 \\
\hline 8496 & 1060 \\
\hline 8869 & 848 \\
\hline 9291 & 944 \\
\hline
\end{tabular}

\begin{tabular}{|c|c|}
\hline \multicolumn{2}{|c|}{4 -core } \\
\hline Nodes & DS-RAI \\
\hline 9555 & 1049 \\
\hline 9925 & 1034 \\
\hline 10145 & 1067 \\
\hline 10518 & 1186 \\
\hline 10555 & 1206 \\
\hline 10639 & 1247 \\
\hline 10993 & 1134 \\
\hline 11465 & 1255 \\
\hline 11727 & 1152 \\
\hline 12170 & 1815 \\
\hline 12397 & 1271 \\
\hline 12442 & 1769 \\
\hline 12697 & 1622 \\
\hline 12975 & 1619 \\
\hline 13006 & 1317 \\
\hline 13608 & 1487 \\
\hline 13689 & 1471 \\
\hline 13862 & 1468 \\
\hline 13912 & 1802 \\
\hline 14025 & 1704 \\
\hline 14047 & 1872 \\
\hline 14886 & 1511 \\
\hline 15692 & 1863 \\
\hline 15723 & 1941 \\
\hline 16224 & 1769 \\
\hline $1 h e \mathrm{DS}$ & $\mathrm{RAI}$ \\
\hline
\end{tabular}

\begin{tabular}{|c|c|}
\hline \multicolumn{2}{|c|}{ 4-core } \\
\hline Nodes & DS-RAI \\
\hline 16337 & 1775 \\
\hline 16365 & 1842 \\
\hline 16392 & 1866 \\
\hline 16570 & 1901 \\
\hline 17318 & 2008 \\
\hline 17583 & 1840 \\
\hline 17878 & 1837 \\
\hline 18530 & 2162 \\
\hline 18985 & 2345 \\
\hline 19712 & 2322 \\
\hline 20064 & 2436 \\
\hline 20123 & 2338 \\
\hline 21430 & 2620 \\
\hline 22661 & 2499 \\
\hline 23047 & 2916 \\
\hline 23511 & 2310 \\
\hline 26435 & 3145 \\
\hline 28272 & 3313 \\
\hline 28486 & 2971 \\
\hline 29483 & 3061 \\
\hline 30046 & 3537 \\
\hline 30750 & 3500 \\
\hline 33712 & 3791 \\
\hline 34844 & 3977 \\
\hline 39081 & 4825 \\
\hline
\end{tabular}

TABLE A.22. Dominating set sizes from the DS-RAI algorithm for the 4-core of Facebook 100 graphs. 


\begin{tabular}{|c|c|}
\hline \multicolumn{2}{|c|}{5 -core } \\
\hline Nodes & DS-RAI \\
\hline 674 & 68 \\
\hline 879 & 97 \\
\hline 1392 & 140 \\
\hline 1399 & 106 \\
\hline 1603 & 150 \\
\hline 2138 & 162 \\
\hline 2154 & 186 \\
\hline 2225 & 176 \\
\hline 2424 & 277 \\
\hline 2515 & 195 \\
\hline 2676 & 189 \\
\hline 2736 & 291 \\
\hline 2792 & 261 \\
\hline 2829 & 234 \\
\hline 2905 & 281 \\
\hline 2968 & 248 \\
\hline 3193 & 281 \\
\hline 3380 & 267 \\
\hline 3387 & 420 \\
\hline 3426 & 316 \\
\hline 3427 & 275 \\
\hline 3653 & 314 \\
\hline 3715 & 280 \\
\hline 3816 & 356 \\
\hline 3867 & 338 \\
\hline
\end{tabular}

\begin{tabular}{|c|c|}
\hline \multicolumn{2}{|c|}{5 -core } \\
\hline Nodes & DS-RAI \\
\hline 4319 & 423 \\
\hline 4687 & 503 \\
\hline 4856 & 413 \\
\hline 5168 & 416 \\
\hline 5746 & 633 \\
\hline 5844 & 623 \\
\hline 5877 & 678 \\
\hline 5898 & 767 \\
\hline 6089 & 634 \\
\hline 6111 & 559 \\
\hline 6182 & 566 \\
\hline 6194 & 633 \\
\hline 6726 & 753 \\
\hline 6909 & 776 \\
\hline 6942 & 694 \\
\hline 7165 & 794 \\
\hline 7278 & 702 \\
\hline 7300 & 638 \\
\hline 7625 & 681 \\
\hline 7870 & 744 \\
\hline 8063 & 797 \\
\hline 8174 & 937 \\
\hline 8369 & 985 \\
\hline 8754 & 792 \\
\hline 9162 & 893 \\
\hline
\end{tabular}

\begin{tabular}{|c|c|}
\hline \multicolumn{2}{|c|}{5 -core } \\
\hline Nodes & DS-RAI \\
\hline 9459 & 991 \\
\hline 9803 & 965 \\
\hline 10065 & 1013 \\
\hline 10297 & 1076 \\
\hline 10342 & 1092 \\
\hline 10470 & 1137 \\
\hline 10883 & 1067 \\
\hline 11318 & 1178 \\
\hline 11635 & 1105 \\
\hline 11848 & 1645 \\
\hline 12162 & 1618 \\
\hline 12308 & 1217 \\
\hline 12443 & 1457 \\
\hline 12733 & 1492 \\
\hline 12867 & 1240 \\
\hline 13424 & 1346 \\
\hline 13499 & 1402 \\
\hline 13654 & 1661 \\
\hline 13676 & 1375 \\
\hline 13782 & 1575 \\
\hline 13802 & 1737 \\
\hline 14769 & 1441 \\
\hline 15417 & 1792 \\
\hline 15502 & 1753 \\
\hline 16052 & 1682 \\
\hline$t h e 1 D S-R A 1$ \\
\hline
\end{tabular}

\begin{tabular}{|c|c|}
\hline \multicolumn{2}{|c|}{5 -core } \\
\hline Nodes & DS-RAI \\
\hline 16159 & 1731 \\
\hline 16174 & 1664 \\
\hline 16228 & 1765 \\
\hline 16408 & 1795 \\
\hline 17108 & 1886 \\
\hline 17356 & 1717 \\
\hline 17746 & 1741 \\
\hline 18238 & 1968 \\
\hline 18662 & 2169 \\
\hline 19449 & 2184 \\
\hline 19716 & 2257 \\
\hline 19890 & 2205 \\
\hline 21077 & 2389 \\
\hline 22413 & 2336 \\
\hline 22679 & 2690 \\
\hline 23326 & 2196 \\
\hline 26107 & 2954 \\
\hline 27848 & 3070 \\
\hline 28221 & 2828 \\
\hline 29219 & 2893 \\
\hline 29683 & 3321 \\
\hline 30372 & 3273 \\
\hline 33323 & 3555 \\
\hline 34518 & 3770 \\
\hline 38475 & 4521 \\
\hline
\end{tabular}

TABLE A.23. Dominating set sizes from the DS-RAI algorithm for the 5-core of Facebook 100 graphs. 


\begin{tabular}{|c|c|c|c|c|c|c|c|}
\hline \multicolumn{2}{|c|}{ 1-core } & \multicolumn{2}{|c|}{ 1-core } & \multicolumn{2}{|c|}{ 1-core } & \multicolumn{2}{|c|}{ 1-core } \\
\hline Nodes & DS-DC & Nodes & DS-DC & Nodes & DS-DC & Nodes & DS-DC \\
\hline 769 & 65 & 4563 & 297 & 10004 & 754 & 17196 & 1215 \\
\hline 962 & 86 & 5075 & 293 & 10521 & 671 & 17212 & 1300 \\
\hline 1446 & 71 & 5180 & 455 & 10567 & 754 & 17425 & 1297 \\
\hline 1518 & 123 & 5372 & 295 & 11247 & 942 & 17444 & 1336 \\
\hline 1659 & 93 & 6386 & 556 & 11509 & 783 & 18163 & 1300 \\
\hline 2235 & 126 & 6440 & 581 & 11621 & 1047 & 18448 & 1127 \\
\hline 2252 & 125 & 6472 & 371 & 11770 & 1034 & 18660 & 1369 \\
\hline 2314 & 126 & 6591 & 596 & 12155 & 724 & 19700 & 1591 \\
\hline 2613 & 146 & 6596 & 474 & 12193 & 924 & 20467 & 1918 \\
\hline 2682 & 267 & 6637 & 514 & 12803 & 779 & 20871 & 1679 \\
\hline 2790 & 142 & 6682 & 524 & 13377 & 1465 & 21325 & 1682 \\
\hline 2920 & 210 & 6833 & 775 & 13653 & 967 & 21679 & 1911 \\
\hline 2970 & 187 & 7324 & 672 & 13686 & 1400 & 22937 & 1896 \\
\hline 2970 & 207 & 7460 & 533 & 13746 & 1316 & 23842 & 1774 \\
\hline 3068 & 176 & 7694 & 657 & 13882 & 1243 & 24389 & 1571 \\
\hline 3075 & 203 & 7752 & 610 & 14070 & 970 & 24580 & 2192 \\
\hline 3445 & 236 & 7755 & 507 & 14916 & 1168 & 27737 & 2155 \\
\hline 3482 & 177 & 7772 & 554 & 14935 & 1308 & 29747 & 2081 \\
\hline 3578 & 217 & 8069 & 511 & 14940 & 1388 & 30147 & 2476 \\
\hline 3593 & 220 & 8578 & 611 & 14948 & 1401 & 30809 & 2150 \\
\hline 3748 & 370 & 8600 & 621 & 15126 & 1306 & 31560 & 2515 \\
\hline 3826 & 196 & 8991 & 839 & 15436 & 1017 & 32375 & 2543 \\
\hline 3898 & 264 & 9069 & 806 & 16516 & 1295 & 35123 & 2509 \\
\hline 4047 & 290 & 9414 & 656 & 16808 & 1495 & 36371 & 2591 \\
\hline 4087 & 247 & 9895 & 684 & 16979 & 1198 & 41554 & 3506 \\
\hline
\end{tabular}

TABLE A.24. Dominating set sizes from the DS-DC algorithm for the 1-core of Facebook 100 graphs. 


\begin{tabular}{|c|c|c|c|c|c|c|c|}
\hline \multicolumn{2}{|c|}{2 -core } & \multicolumn{2}{|c|}{ 2-core } & \multicolumn{2}{|c|}{ 2-core } & \multicolumn{2}{|c|}{ 2-core } \\
\hline Nodes & DS-DC & Nodes & DS-DC & Nodes & DS-DC & Nodes & DS-DC \\
\hline 734 & 51 & 4484 & 251 & 9800 & 661 & 16801 & 998 \\
\hline 927 & 70 & 4979 & 331 & 10270 & 593 & 16832 & 1099 \\
\hline 1428 & 59 & 5001 & 258 & 10356 & 580 & 16942 & 1091 \\
\hline 1468 & 94 & 5291 & 251 & 10996 & 800 & 17032 & 1086 \\
\hline 1644 & 82 & 6129 & 411 & 11087 & 756 & 17799 & 1092 \\
\hline 2199 & 104 & 6194 & 457 & 11185 & 782 & 18138 & 1113 \\
\hline 2214 & 107 & 6318 & 447 & 11253 & 644 & 18187 & 969 \\
\hline 2281 & 110 & 6371 & 324 & 11842 & 771 & 19176 & 1328 \\
\hline 2575 & 123 & 6392 & 362 & 11951 & 613 & 19818 & 1581 \\
\hline 2589 & 221 & 6426 & 407 & 12636 & 688 & 20311 & 1387 \\
\hline 2743 & 120 & 6490 & 413 & 12892 & 1218 & 20789 & 1408 \\
\hline 2857 & 169 & 6514 & 622 & 13171 & 1118 & 20892 & 1520 \\
\hline 2910 & 151 & 7104 & 541 & 13277 & 1062 & 22243 & 1536 \\
\hline 2915 & 184 & 7260 & 440 & 13376 & 835 & 23326 & 1508 \\
\hline 3013 & 163 & 7370 & 492 & 13511 & 1044 & 23902 & 1815 \\
\hline 3034 & 159 & 7562 & 525 & 13868 & 850 & 23966 & 1346 \\
\hline 3342 & 176 & 7570 & 443 & 14398 & 898 & 27175 & 1906 \\
\hline 3439 & 151 & 7578 & 411 & 14443 & 943 & 29184 & 1789 \\
\hline 3522 & 178 & 7903 & 421 & 14503 & 1170 & 29288 & 2038 \\
\hline 3526 & 186 & 8292 & 477 & 14533 & 1107 & 30203 & 1829 \\
\hline 3629 & 315 & 8380 & 505 & 14600 & 1222 & 30898 & 2180 \\
\hline 3794 & 206 & 8716 & 698 & 15199 & 896 & 31640 & 2148 \\
\hline 3795 & 176 & 8813 & 661 & 16168 & 1110 & 34514 & 2167 \\
\hline 3956 & 231 & 9141 & 518 & 16342 & 1246 & 35694 & 2253 \\
\hline 4006 & 218 & 9621 & 519 & 16638 & 1013 & 40458 & 2927 \\
\hline
\end{tabular}

TABLE A.25. Dominating set sizes from the DS-DC algorithm for the 2-core of Facebook 100 graphs. 


\begin{tabular}{|c|c|c|c|c|c|c|c|}
\hline \multicolumn{2}{|c|}{3 -core } & \multicolumn{2}{|c|}{ 3-core } & \multicolumn{2}{|c|}{ 3-core } & \multicolumn{2}{|c|}{3 -core } \\
\hline Nodes & DS-DC & Nodes & DS-DC & Nodes & DS-DC & Nodes & DS-DC \\
\hline 699 & 43 & 4415 & 225 & 9665 & 607 & 16540 & 884 \\
\hline 906 & 64 & 4840 & 273 & 10082 & 508 & 16587 & 985 \\
\hline 1414 & 56 & 4945 & 237 & 10243 & 536 & 16637 & 975 \\
\hline 1438 & 85 & 5242 & 231 & 10767 & 635 & 16785 & 985 \\
\hline 1630 & 73 & 5953 & 338 & 10816 & 741 & 17536 & 1024 \\
\hline 2176 & 94 & 6061 & 401 & 10852 & 667 & 17811 & 981 \\
\hline 2192 & 97 & 6153 & 392 & 11108 & 594 & 18022 & 882 \\
\hline 2264 & 105 & 6266 & 515 & 11624 & 673 & 18808 & 1175 \\
\hline 2526 & 193 & 6275 & 319 & 11833 & 563 & 19342 & 1365 \\
\hline 2548 & 107 & 6281 & 356 & 12495 & 1054 & 19998 & 1266 \\
\hline 2719 & 110 & 6308 & 298 & 12502 & 620 & 20405 & 1314 \\
\hline 2817 & 156 & 6379 & 365 & 12777 & 960 & 20432 & 1258 \\
\hline 2863 & 155 & 6964 & 469 & 12962 & 926 & 21800 & 1337 \\
\hline 2880 & 145 & 7121 & 376 & 13174 & 752 & 22964 & 1368 \\
\hline 2971 & 142 & 7180 & 407 & 13242 & 936 & 23440 & 1637 \\
\hline 3005 & 146 & 7403 & 448 & 13734 & 804 & 23718 & 1235 \\
\hline 3276 & 143 & 7444 & 352 & 14002 & 783 & 26771 & 1740 \\
\hline 3424 & 143 & 7457 & 392 & 14105 & 779 & 28750 & 1825 \\
\hline 3484 & 165 & 7802 & 374 & 14209 & 1071 & 28796 & 1610 \\
\hline 3491 & 169 & 8114 & 404 & 14272 & 998 & 29781 & 1669 \\
\hline 3544 & 285 & 8250 & 452 & 14299 & 1117 & 30457 & 2020 \\
\hline 3741 & 183 & 8517 & 616 & 15031 & 836 & 31157 & 1949 \\
\hline 3774 & 171 & 8642 & 587 & 15925 & 1007 & 34080 & 1987 \\
\hline 3902 & 208 & 8984 & 463 & 16008 & 1117 & 35240 & 2059 \\
\hline 3954 & 198 & 9459 & 463 & 16408 & 911 & 39703 & 2618 \\
\hline
\end{tabular}

TABLE A.26. Dominating set sizes from the DS-DC algorithm for the 3-core of Facebook 100 graphs. 


\begin{tabular}{|c|c|c|c|c|c|c|c|}
\hline \multicolumn{2}{|c|}{ 4-core } & \multicolumn{2}{|c|}{ 4-core } & \multicolumn{2}{|c|}{ 4-core } & \multicolumn{2}{|c|}{ 4-core } \\
\hline Nodes & DS-DC & Nodes & DS-DC & Nodes & DS-DC & Nodes & DS-DC \\
\hline 684 & 35 & 4364 & 208 & 9555 & 563 & 16337 & 829 \\
\hline 891 & 61 & 4765 & 254 & 9925 & 442 & 16365 & 883 \\
\hline 1408 & 56 & 4895 & 224 & 10145 & 495 & 16392 & 928 \\
\hline 1411 & 78 & 5203 & 215 & 10518 & 562 & 16570 & 903 \\
\hline 1614 & 71 & 5842 & 304 & 10555 & 580 & 17318 & 941 \\
\hline 2153 & 85 & 5954 & 366 & 10639 & 675 & 17583 & 898 \\
\hline 2170 & 92 & 5994 & 340 & 10993 & 548 & 17878 & 832 \\
\hline 2241 & 92 & 6070 & 441 & 11465 & 604 & 18530 & 1084 \\
\hline 2470 & 171 & 6181 & 294 & 11727 & 526 & 18985 & 1253 \\
\hline 2530 & 103 & 6189 & 333 & 12170 & 945 & 19712 & 1166 \\
\hline 2701 & 105 & 6235 & 282 & 12397 & 588 & 20064 & 1194 \\
\hline 2772 & 150 & 6291 & 339 & 12442 & 848 & 20123 & 1165 \\
\hline 2831 & 146 & 6840 & 432 & 12697 & 831 & 21430 & 1230 \\
\hline 2851 & 132 & 7020 & 340 & 12975 & 861 & 22661 & 1262 \\
\hline 2936 & 133 & 7039 & 365 & 13006 & 680 & 23047 & 1508 \\
\hline 2990 & 148 & 7286 & 404 & 13608 & 745 & 23511 & 1147 \\
\hline 3224 & 134 & 7367 & 362 & 13689 & 696 & 26435 & 1624 \\
\hline 3404 & 136 & 7372 & 328 & 13862 & 712 & 28272 & 1650 \\
\hline 3455 & 157 & 7701 & 338 & 13912 & 967 & 28486 & 1503 \\
\hline 3460 & 158 & 7970 & 356 & 14025 & 886 & 29483 & 1563 \\
\hline 3462 & 260 & 8131 & 412 & 14047 & 1036 & 30046 & 1902 \\
\hline 3692 & 169 & 8351 & 556 & 14886 & 791 & 30750 & 1807 \\
\hline 3744 & 162 & 8496 & 544 & 15692 & 931 & 33712 & 1857 \\
\hline 3858 & 190 & 8869 & 430 & 15723 & 1027 & 34844 & 1916 \\
\hline 3905 & 178 & 9291 & 420 & 16224 & 836 & 39081 & 2407 \\
\hline
\end{tabular}

TABLE A.27. Dominating set sizes from the DS-DC algorithm for the 4-core of Facebook 100 graphs. 


\begin{tabular}{|c|c|c|c|c|c|c|c|}
\hline \multicolumn{2}{|c|}{ 5-core } & \multicolumn{2}{|c|}{ 5-core } & \multicolumn{2}{|c|}{ 5-core } & \multicolumn{2}{|c|}{ 5-core } \\
\hline Nodes & DS-DC & Nodes & DS-DC & Nodes & DS-DC & Nodes & DS-DC \\
\hline 674 & 33 & 4319 & 191 & 9459 & 534 & 16159 & 810 \\
\hline 879 & 56 & 4687 & 230 & 9803 & 407 & 16174 & 787 \\
\hline 1392 & 69 & 4856 & 211 & 10065 & 472 & 16228 & 886 \\
\hline 1399 & 51 & 5168 & 204 & 10297 & 491 & 16408 & 853 \\
\hline 1603 & 69 & 5746 & 282 & 10342 & 523 & 17108 & 880 \\
\hline 2138 & 80 & 5844 & 334 & 10470 & 624 & 17356 & 842 \\
\hline 2154 & 90 & 5877 & 303 & 10883 & 509 & 17746 & 786 \\
\hline 2225 & 90 & 5898 & 403 & 11318 & 562 & 18238 & 1000 \\
\hline 2424 & 158 & 6089 & 307 & 11635 & 503 & 18662 & 1156 \\
\hline 2515 & 96 & 6111 & 275 & 11848 & 866 & 19449 & 1078 \\
\hline 2676 & 91 & 6182 & 264 & 12162 & 770 & 19716 & 1082 \\
\hline 2736 & 137 & 6194 & 312 & 12308 & 547 & 19890 & 1081 \\
\hline 2792 & 138 & 6726 & 403 & 12443 & 760 & 21077 & 1122 \\
\hline 2829 & 125 & 6909 & 320 & 12733 & 794 & 22413 & 1190 \\
\hline 2905 & 124 & 6942 & 323 & 12867 & 642 & 22679 & 1406 \\
\hline 2968 & 137 & 7165 & 380 & 13424 & 630 & 23326 & 1106 \\
\hline 3193 & 126 & 7278 & 338 & 13499 & 717 & 26107 & 1552 \\
\hline 3380 & 126 & 7300 & 303 & 13654 & 878 & 27848 & 1536 \\
\hline 3387 & 242 & 7625 & 316 & 13676 & 651 & 28221 & 1428 \\
\hline 3426 & 150 & 7870 & 336 & 13782 & 822 & 29219 & 1492 \\
\hline 3427 & 154 & 8063 & 390 & 13802 & 960 & 29683 & 1792 \\
\hline 3653 & 155 & 8174 & 512 & 14769 & 756 & 30372 & 1679 \\
\hline 3715 & 154 & 8369 & 502 & 15417 & 944 & 33323 & 1744 \\
\hline 3816 & 173 & 8754 & 406 & 15502 & 865 & 34518 & 1804 \\
\hline 3867 & 166 & 9162 & 381 & 16052 & 782 & 38475 & 2228 \\
\hline
\end{tabular}

TABLE A.28. Dominating set sizes from the DS-DC algorithm for the 5-core of Facebook 100 graphs. 


\section{A.3. DMGEO-P Data Tables}

\begin{tabular}{|c|c|c|c|c|c|c|}
\hline Dataset Name & Nodes & Edges & Min Degree & Avg Degree & Max Degree & Density \\
\hline Caltech36 & 769 & 4142 & 1 & 11 & 28 & 0.014 \\
\hline Reed98 & 962 & 7564 & 4 & 16 & 68 & 0.016 \\
\hline Haverford76 & 1446 & 14927 & 8 & 21 & 52 & 0.014 \\
\hline Simmons81 & 1518 & 10300 & 2 & 14 & 61 & 0.009 \\
\hline Swarthmore42 & 1659 & 21837 & 9 & 26 & 78 & 0.016 \\
\hline Amherst41 & 2235 & 32673 & 9 & 29 & 130 & 0.013 \\
\hline Bowdoin47 & 2252 & 23851 & 8 & 21 & 86 & 0.009 \\
\hline Hamilton46 & 2314 & 30769 & 9 & 27 & 134 & 0.011 \\
\hline Trinity100 & 2613 & 30343 & 8 & 23 & 95 & 0.009 \\
\hline USFCA72 & 2682 & 22240 & 4 & 17 & 114 & 0.006 \\
\hline Williams40 & 2790 & 32770 & 8 & 23 & 112 & 0.008 \\
\hline Oberlin44 & 2920 & 28940 & 4 & 20 & 93 & 0.007 \\
\hline Smith60 & 2970 & 26589 & 3 & 18 & 68 & 0.006 \\
\hline Wellesley22 & 2970 & 29984 & 5 & 20 & 105 & 0.007 \\
\hline Vassar85 & 3068 & 32678 & 7 & 21 & 94 & 0.007 \\
\hline Middlebury45 & 3075 & 34063 & 6 & 22 & 71 & 0.007 \\
\hline Pepperdine86 & 3445 & 44192 & 8 & 26 & 112 & 0.007 \\
\hline Colgate88 & 3482 & 42672 & 10 & 25 & 92 & 0.007 \\
\hline Santa74 & 3578 & 42130 & 8 & 24 & 79 & 0.007 \\
\hline Wesleyan43 & 3593 & 43480 & 7 & 24 & 122 & 0.007 \\
\hline Mich67 & 3748 & 21174 & 1 & 11 & 52 & 0.003 \\
\hline Bucknell39 & 3826 & 46528 & 9 & 24 & 93 & 0.006 \\
\hline Brandeis99 & 3898 & 36284 & 4 & 19 & 91 & 0.005 \\
\hline Howard90 & 4047 & 56430 & 11 & 28 & 103 & 0.007 \\
\hline Rice31 & 4087 & 49878 & 9 & 24 & 84 & 0.006 \\
\hline Rochester38 & 4563 & 37545 & 3 & 16 & 86 & 0.004 \\
\hline Lehigh96 & 5075 & 53745 & 4 & 21 & 73 & 0.004 \\
\hline Johns-Hopkins55 & 5180 & 44719 & 4 & 17 & 102 & 0.003 \\
\hline Wake73 & 5372 & 78506 & 10 & 29 & 134 & 0.005 \\
\hline American75 & 6386 & 55080 & 2 & 17 & 115 & 0.003 \\
\hline MIT8 & 6440 & 58792 & 4 & 18 & 98 & 0.003 \\
\hline William77 & 6472 & 64734 & 5 & 20 & 91 & 0.003 \\
\hline UChicago30 & 6591 & 53290 & 3 & 16 & 108 & 0.002 \\
\hline
\end{tabular}

TABLE A.29. General graph properties computed for DMGEO-P Facebook 100 samples. 


\begin{tabular}{|c|c|c|c|c|c|c|}
\hline Dataset Name & Nodes & Edges & Min Degree & Avg Degree & Max Degree & Density \\
\hline Princeton12 & 6596 & 71730 & 5 & 22 & 116 & 0.003 \\
\hline Carnegie49 & 6637 & 62763 & 5 & 19 & 131 & 0.003 \\
\hline Tufts18 & 6682 & 59879 & 5 & 18 & 80 & 0.003 \\
\hline UC64 & 6833 & 36166 & 1 & 11 & 41 & 0.002 \\
\hline Vermont70 & 7324 & 48241 & 1 & 13 & 93 & 0.002 \\
\hline Emory27 & 7460 & 80004 & 4 & 21 & 81 & 0.003 \\
\hline Dartmouth6 & 7694 & 74083 & 5 & 19 & 104 & 0.003 \\
\hline Tulane29 & 7752 & 63654 & 2 & 16 & 70 & 0.002 \\
\hline WashU32 & 7755 & 84762 & 6 & 22 & 139 & 0.003 \\
\hline Villanova62 & 7772 & 74730 & 4 & 19 & 102 & 0.002 \\
\hline Vanderbilt48 & 8069 & 104241 & 4 & 26 & 155 & 0.003 \\
\hline Yale4 & 8578 & 93824 & 6 & 22 & 102 & 0.003 \\
\hline Brown11 & 8600 & 102424 & 5 & 24 & 170 & 0.003 \\
\hline UCSC68 & 8991 & 55744 & 1 & 12 & 79 & 0.001 \\
\hline Maine59 & 9069 & 48694 & 1 & 11 & 61 & 0.001 \\
\hline Georgetown15 & 9414 & 107699 & 5 & 23 & 159 & 0.002 \\
\hline Duke14 & 9895 & 117759 & 6 & 24 & 108 & 0.002 \\
\hline Bingham82 & 10004 & 88035 & 3 & 18 & 75 & 0.002 \\
\hline Mississippi66 & 10521 & 155807 & 9 & 30 & 152 & 0.003 \\
\hline Northwestern25 & 10567 & 108453 & 4 & 21 & 123 & 0.002 \\
\hline Cal65 & 11247 & 74532 & 2 & 13 & 52 & 0.001 \\
\hline BC17 & 11509 & 113743 & 4 & 20 & 119 & 0.002 \\
\hline Stanford3 & 11621 & 134172 & 7 & 23 & 121 & 0.002 \\
\hline Columbia2 & 11770 & 105214 & 4 & 18 & 205 & 0.002 \\
\hline Notre-Dame57 & 12155 & 123570 & 4 & 20 & 164 & 0.002 \\
\hline GWU54 & 12193 & 104158 & 3 & 17 & 141 & 0.001 \\
\hline Baylor93 & 12803 & 155582 & 6 & 24 & 132 & 0.002 \\
\hline USF51 & 13377 & 82776 & 1 & 12 & 104 & 0.001 \\
\hline Syracuse56 & 13653 & 126100 & 4 & 18 & 88 & 0.001 \\
\hline Temple83 & 13686 & 81345 & 1 & 12 & 135 & 0.001 \\
\hline UC61 & 13746 & 111133 & 1 & 16 & 111 & 0.001 \\
\hline Northeastern19 & 13882 & 98728 & 2 & 14 & 134 & 0.001 \\
\hline TABLE A.30 & Gener & 9 & & 51 & \\
\hline
\end{tabular}

TABLE A.30. General graph properties computed for DMGEO-P Facebook 100 samples (continued). 


\begin{tabular}{|c|c|c|c|c|c|c|}
\hline Dataset Name & Nodes & Edges & Min Degree & Avg Degree & Max Degree & Density \\
\hline JMU79 & 14070 & 100381 & 2 & 14 & 102 & 0.001 \\
\hline UPenn7 & 14916 & 173402 & 6 & 23 & 119 & 0.002 \\
\hline UCSB37 & 14935 & 111503 & 3 & 15 & 95 & 0.001 \\
\hline UCF52 & 14940 & 87574 & 0 & 12 & 64 & 0.001 \\
\hline UCSD34 & 14948 & 92593 & 0 & 12 & 89 & 0.001 \\
\hline Harvard1 & 15126 & 198767 & 5 & 26 & 122 & 0.002 \\
\hline MU78 & 15436 & 126959 & 2 & 16 & 99 & 0.001 \\
\hline UMass92 & 16516 & 123744 & 2 & 15 & 150 & 0.001 \\
\hline UC33 & 16808 & 122404 & 1 & 15 & 109 & 0.001 \\
\hline Tennessee95 & 16979 & 166551 & 4 & 20 & 161 & 0.001 \\
\hline UVA16 & 17196 & 173907 & 3 & 20 & 128 & 0.001 \\
\hline UConn91 & 17212 & 133615 & 2 & 16 & 107 & 0.001 \\
\hline Oklahoma97 & 17425 & 230563 & 7 & 26 & 169 & 0.002 \\
\hline USC35 & 17444 & 151482 & 3 & 17 & 82 & 0.001 \\
\hline UNC28 & 18163 & 154789 & 3 & 17 & 178 & 0.001 \\
\hline Auburn71 & 18448 & 202971 & 4 & 22 & 188 & 0.001 \\
\hline Cornell5 & 18660 & 166569 & 2 & 18 & 122 & 0.001 \\
\hline BU10 & 19700 & 131217 & 1 & 13 & 82 & 0.001 \\
\hline UCLA26 & 20467 & 163294 & 2 & 16 & 79 & 0.001 \\
\hline Maryland58 & 20871 & 157377 & 2 & 15 & 99 & 0.001 \\
\hline Virginia63 & 21325 & 161292 & 2 & 15 & 159 & 0.001 \\
\hline NYU9 & 21679 & 179477 & 2 & 17 & 243 & 0.001 \\
\hline Berkeley13 & 22937 & 206236 & 3 & 18 & 229 & 0.001 \\
\hline Wisconsin 87 & 23842 & 186132 & 2 & 16 & 159 & 0.001 \\
\hline UGA50 & 24389 & 232397 & 2 & 19 & 108 & 0.001 \\
\hline Rutgers89 & 24580 & 162784 & 2 & 13 & 106 & 0.001 \\
\hline FSU53 & 27737 & 206406 & 1 & 15 & 83 & 0.001 \\
\hline Indiana69 & 29747 & 261927 & 2 & 18 & 120 & 0.001 \\
\hline Michigan23 & 30147 & 270614 & 3 & 18 & 139 & 0.001 \\
\hline UIllinois20 & 30809 & 269799 & 3 & 18 & 108 & 0.001 \\
\hline Texas80 & 31560 & 227070 & 1 & 14 & 95 & 0.000 \\
\hline MSU24 & 32375 & 244658 & 1 & 15 & 139 & 0.000 \\
\hline UF21 & 35123 & 316021 & 2 & 18 & 205 & 0.001 \\
\hline Texas84 & 36371 & 368676 & 4 & 20 & 198 & 0.001 \\
\hline Penn94 & 41554 & 288212 & 1 & 14 & 245 & 0.000 \\
\hline
\end{tabular}

TABLE A.31. General graph properties computed for DMGEO-P Facebook 100 samples (continued). 


\begin{tabular}{|c|c|}
\hline \multicolumn{2}{|c|}{ 0-core } \\
\hline Nodes & DS-RAI \\
\hline 769 & 153 \\
\hline 962 & 119 \\
\hline 1446 & 171 \\
\hline 1518 & 214 \\
\hline 1659 & 142 \\
\hline 2235 & 180 \\
\hline 2252 & 248 \\
\hline 2314 & 209 \\
\hline 2613 & 257 \\
\hline 2682 & 337 \\
\hline 2790 & 281 \\
\hline 2920 & 325 \\
\hline 2970 & 384 \\
\hline 2970 & 337 \\
\hline 3068 & 337 \\
\hline 3075 & 345 \\
\hline 3445 & 320 \\
\hline 3482 & 353 \\
\hline 3578 & 370 \\
\hline 3593 & 344 \\
\hline 3748 & 682 \\
\hline 3826 & 378 \\
\hline 3898 & 487 \\
\hline 4047 & 378 \\
\hline 4087 & 413 \\
\hline
\end{tabular}

\begin{tabular}{|c|c|}
\hline \multicolumn{2}{|c|}{ 0-core } \\
\hline Nodes & DS-RAI \\
\hline 4563 & 612 \\
\hline 5075 & 561 \\
\hline 5180 & 687 \\
\hline 5372 & 452 \\
\hline 6386 & 823 \\
\hline 6440 & 796 \\
\hline 6472 & 769 \\
\hline 6591 & 901 \\
\hline 6596 & 711 \\
\hline 6637 & 842 \\
\hline 6682 & 854 \\
\hline 6833 & 1273 \\
\hline 7324 & 1172 \\
\hline 7460 & 850 \\
\hline 7694 & 928 \\
\hline 7752 & 1081 \\
\hline 7755 & 859 \\
\hline 7772 & 955 \\
\hline 8069 & 795 \\
\hline 8578 & 946 \\
\hline 8600 & 875 \\
\hline 8991 & 1502 \\
\hline 9069 & 1748 \\
\hline 9414 & 1008 \\
\hline 9895 & 1035 \\
\hline
\end{tabular}

\begin{tabular}{|c|c|}
\hline \multicolumn{2}{|c|}{0 -core } \\
\hline Nodes & DS-RAI \\
\hline 10004 & 1292 \\
\hline 10521 & 937 \\
\hline 10567 & 1249 \\
\hline 11247 & 1839 \\
\hline 11509 & 1407 \\
\hline 11621 & 1239 \\
\hline 11770 & 1466 \\
\hline 12155 & 1402 \\
\hline 12193 & 1607 \\
\hline 12803 & 1319 \\
\hline 13377 & 2240 \\
\hline 13653 & 1760 \\
\hline 13686 & 2323 \\
\hline 13746 & 1883 \\
\hline 13882 & 2060 \\
\hline 14070 & 2161 \\
\hline 14916 & 1598 \\
\hline 14935 & 2164 \\
\hline 14940 & 2622 \\
\hline 14948 & 2520 \\
\hline 15126 & 1458 \\
\hline 15436 & 2195 \\
\hline 16516 & 2434 \\
\hline 16808 & 2533 \\
\hline 16979 & 2030 \\
\hline $1 h e D S-R A 1$ \\
\hline
\end{tabular}

\begin{tabular}{|c|c|}
\hline \multicolumn{2}{|c|}{0 -core } \\
\hline Nodes & DS-RAI \\
\hline 17196 & 2063 \\
\hline 17212 & 2467 \\
\hline 17425 & 1665 \\
\hline 17444 & 2392 \\
\hline 18163 & 2403 \\
\hline 18448 & 2047 \\
\hline 18660 & 2420 \\
\hline 19700 & 3204 \\
\hline 20467 & 2948 \\
\hline 20871 & 3083 \\
\hline 21325 & 3096 \\
\hline 21679 & 2859 \\
\hline 22937 & 2880 \\
\hline 23842 & 3371 \\
\hline 24389 & 3057 \\
\hline 24580 & 3973 \\
\hline 27737 & 4188 \\
\hline 29747 & 3943 \\
\hline 30147 & 3872 \\
\hline 30809 & 4123 \\
\hline 31560 & 4854 \\
\hline 32375 & 4750 \\
\hline 35123 & 4551 \\
\hline 36371 & 4265 \\
\hline 41554 & 6336 \\
\hline
\end{tabular}

TABLE A.32. Dominating set sizes from the DS-RAI algorithm for the 0-core of DMGEO-P Facebook 100 graphs. 


\begin{tabular}{|c|c|}
\hline \multicolumn{2}{|c|}{ 1-core } \\
\hline Nodes & DS-RAI \\
\hline 769 & 153 \\
\hline 962 & 119 \\
\hline 1446 & 171 \\
\hline 1518 & 214 \\
\hline 1659 & 142 \\
\hline 2235 & 180 \\
\hline 2252 & 248 \\
\hline 2314 & 209 \\
\hline 2613 & 257 \\
\hline 2682 & 337 \\
\hline 2790 & 281 \\
\hline 2920 & 325 \\
\hline 2970 & 384 \\
\hline 2970 & 337 \\
\hline 3068 & 337 \\
\hline 3075 & 345 \\
\hline 3445 & 320 \\
\hline 3482 & 353 \\
\hline 3578 & 370 \\
\hline 3593 & 344 \\
\hline 3748 & 682 \\
\hline 3826 & 378 \\
\hline 3898 & 487 \\
\hline 4047 & 378 \\
\hline 4087 & 413 \\
\hline
\end{tabular}

\begin{tabular}{|c|c|}
\hline \multicolumn{2}{|c|}{ 1-core } \\
\hline Nodes & DS-RAI \\
\hline 4563 & 612 \\
\hline 5075 & 561 \\
\hline 5180 & 687 \\
\hline 5372 & 452 \\
\hline 6386 & 823 \\
\hline 6440 & 796 \\
\hline 6472 & 769 \\
\hline 6591 & 901 \\
\hline 6596 & 711 \\
\hline 6637 & 842 \\
\hline 6682 & 854 \\
\hline 6833 & 1273 \\
\hline 7324 & 1172 \\
\hline 7460 & 850 \\
\hline 7694 & 928 \\
\hline 7752 & 1081 \\
\hline 7755 & 859 \\
\hline 7772 & 955 \\
\hline 8069 & 795 \\
\hline 8578 & 946 \\
\hline 8600 & 875 \\
\hline 8991 & 1502 \\
\hline 9069 & 1748 \\
\hline 9414 & 1008 \\
\hline 9895 & 1035 \\
\hline
\end{tabular}

\begin{tabular}{|c|c|}
\hline \multicolumn{2}{|c|}{1 -core } \\
\hline Nodes & DS-RAI \\
\hline 10004 & 1292 \\
\hline 10521 & 937 \\
\hline 10567 & 1249 \\
\hline 11247 & 1839 \\
\hline 11509 & 1407 \\
\hline 11621 & 1239 \\
\hline 11770 & 1466 \\
\hline 12155 & 1402 \\
\hline 12193 & 1607 \\
\hline 12803 & 1319 \\
\hline 13377 & 2240 \\
\hline 13653 & 1760 \\
\hline 13686 & 2323 \\
\hline 13746 & 1883 \\
\hline 13882 & 2060 \\
\hline 14070 & 2161 \\
\hline 14916 & 1598 \\
\hline 14935 & 2164 \\
\hline 14939 & 2621 \\
\hline 14947 & 2519 \\
\hline 15126 & 1458 \\
\hline 15436 & 2195 \\
\hline 16516 & 2434 \\
\hline 16808 & 2533 \\
\hline 16979 & 2030 \\
\hline $1 h e D S-R A 1$ \\
\hline
\end{tabular}

\begin{tabular}{|c|c|}
\hline \multicolumn{2}{|c|}{1 -core } \\
\hline Nodes & DS-RAI \\
\hline 17196 & 2063 \\
\hline 17212 & 2467 \\
\hline 17425 & 1665 \\
\hline 17444 & 2392 \\
\hline 18163 & 2403 \\
\hline 18448 & 2047 \\
\hline 18660 & 2420 \\
\hline 19700 & 3204 \\
\hline 20467 & 2948 \\
\hline 20871 & 3083 \\
\hline 21325 & 3096 \\
\hline 21679 & 2859 \\
\hline 22937 & 2880 \\
\hline 23842 & 3371 \\
\hline 24389 & 3057 \\
\hline 24580 & 3973 \\
\hline 27737 & 4188 \\
\hline 29747 & 3943 \\
\hline 30147 & 3872 \\
\hline 30809 & 4123 \\
\hline 31560 & 4854 \\
\hline 32375 & 4750 \\
\hline 35123 & 4551 \\
\hline 36371 & 4265 \\
\hline 41554 & 6336 \\
\hline
\end{tabular}

TABLE A.33. Dominating set sizes from the DS-RAI algorithm for the 1-core of DMGEO-P Facebook 100 graphs. 


\begin{tabular}{|c|c|}
\hline \multicolumn{2}{|c|}{2 -core } \\
\hline Nodes & DS-RAI \\
\hline 768 & 152 \\
\hline 962 & 119 \\
\hline 1446 & 171 \\
\hline 1518 & 214 \\
\hline 1659 & 142 \\
\hline 2235 & 180 \\
\hline 2252 & 248 \\
\hline 2314 & 209 \\
\hline 2613 & 257 \\
\hline 2682 & 337 \\
\hline 2790 & 281 \\
\hline 2920 & 325 \\
\hline 2970 & 384 \\
\hline 2970 & 337 \\
\hline 3068 & 337 \\
\hline 3075 & 345 \\
\hline 3445 & 320 \\
\hline 3482 & 353 \\
\hline 3578 & 370 \\
\hline 3593 & 344 \\
\hline 3745 & 680 \\
\hline 3826 & 378 \\
\hline 3898 & 487 \\
\hline 4047 & 378 \\
\hline 4087 & 413 \\
\hline
\end{tabular}

\begin{tabular}{|c|c|}
\hline \multicolumn{2}{|c|}{2 -core } \\
\hline Nodes & DS-RAI \\
\hline 4563 & 612 \\
\hline 5075 & 561 \\
\hline 5180 & 687 \\
\hline 5372 & 452 \\
\hline 6386 & 823 \\
\hline 6440 & 796 \\
\hline 6472 & 769 \\
\hline 6591 & 901 \\
\hline 6596 & 711 \\
\hline 6637 & 842 \\
\hline 6682 & 854 \\
\hline 6832 & 1272 \\
\hline 7322 & 1170 \\
\hline 7460 & 850 \\
\hline 7694 & 928 \\
\hline 7752 & 1081 \\
\hline 7755 & 859 \\
\hline 7772 & 955 \\
\hline 8069 & 795 \\
\hline 8578 & 946 \\
\hline 8600 & 875 \\
\hline 8989 & 1500 \\
\hline 9064 & 1745 \\
\hline 9414 & 1008 \\
\hline 9895 & 1035 \\
\hline
\end{tabular}

\begin{tabular}{|c|c|}
\hline \multicolumn{2}{|c|}{2 -core } \\
\hline Nodes & DS-RAI \\
\hline 10004 & 1292 \\
\hline 10521 & 937 \\
\hline 10567 & 1249 \\
\hline 11247 & 1839 \\
\hline 11509 & 1407 \\
\hline 11621 & 1239 \\
\hline 11770 & 1466 \\
\hline 12155 & 1402 \\
\hline 12193 & 1607 \\
\hline 12803 & 1319 \\
\hline 13372 & 2237 \\
\hline 13653 & 1760 \\
\hline 13681 & 2320 \\
\hline 13745 & 1882 \\
\hline 13882 & 2060 \\
\hline 14070 & 2161 \\
\hline 14916 & 1598 \\
\hline 14935 & 2164 \\
\hline 14936 & 2619 \\
\hline 14941 & 2515 \\
\hline 15126 & 1458 \\
\hline 15436 & 2195 \\
\hline 16516 & 2434 \\
\hline 16807 & 2533 \\
\hline 16979 & 2030 \\
\hline $1 h+D S-R A I$ \\
\hline
\end{tabular}

\begin{tabular}{|c|c|}
\hline \multicolumn{2}{|c|}{2 -core } \\
\hline Nodes & DS-RAI \\
\hline 17196 & 2063 \\
\hline 17212 & 2467 \\
\hline 17425 & 1665 \\
\hline 17444 & 2392 \\
\hline 18163 & 2403 \\
\hline 18448 & 2047 \\
\hline 18660 & 2420 \\
\hline 19699 & 3203 \\
\hline 20467 & 2948 \\
\hline 20871 & 3083 \\
\hline 21325 & 3096 \\
\hline 21679 & 2859 \\
\hline 22937 & 2880 \\
\hline 23842 & 3371 \\
\hline 24389 & 3057 \\
\hline 24580 & 3973 \\
\hline 27736 & 4187 \\
\hline 29747 & 3943 \\
\hline 30147 & 3872 \\
\hline 30809 & 4123 \\
\hline 31559 & 4853 \\
\hline 32373 & 4748 \\
\hline 35123 & 4551 \\
\hline 36371 & 4265 \\
\hline 41552 & 6334 \\
\hline
\end{tabular}

TABLE A.34. Dominating set sizes from the DS-RAI algorithm for the 2-core of DMGEO-P Facebook 100 graphs. 


\begin{tabular}{|c|c|}
\hline \multicolumn{2}{|c|}{3 -core } \\
\hline Nodes & DS-RAI \\
\hline 764 & 149 \\
\hline 962 & 119 \\
\hline 1446 & 171 \\
\hline 1517 & 214 \\
\hline 1659 & 142 \\
\hline 2235 & 180 \\
\hline 2252 & 248 \\
\hline 2314 & 209 \\
\hline 2613 & 257 \\
\hline 2682 & 337 \\
\hline 2790 & 281 \\
\hline 2920 & 325 \\
\hline 2970 & 384 \\
\hline 2970 & 337 \\
\hline 3068 & 337 \\
\hline 3075 & 345 \\
\hline 3445 & 320 \\
\hline 3482 & 353 \\
\hline 3578 & 370 \\
\hline 3593 & 344 \\
\hline 3735 & 675 \\
\hline 3826 & 378 \\
\hline 3898 & 487 \\
\hline 4047 & 378 \\
\hline 4087 & 413 \\
\hline
\end{tabular}

\begin{tabular}{|c|c|}
\hline \multicolumn{2}{|c|}{3 -core } \\
\hline Nodes & DS-RAI \\
\hline 4563 & 612 \\
\hline 5075 & 561 \\
\hline 5180 & 687 \\
\hline 5372 & 452 \\
\hline 6385 & 822 \\
\hline 6440 & 796 \\
\hline 6472 & 769 \\
\hline 6591 & 901 \\
\hline 6596 & 711 \\
\hline 6637 & 842 \\
\hline 6682 & 854 \\
\hline 6812 & 1258 \\
\hline 7321 & 1170 \\
\hline 7460 & 850 \\
\hline 7694 & 928 \\
\hline 7751 & 1084 \\
\hline 7755 & 859 \\
\hline 7772 & 955 \\
\hline 8069 & 795 \\
\hline 8578 & 946 \\
\hline 8600 & 875 \\
\hline 8983 & 1497 \\
\hline 9051 & 1739 \\
\hline 9414 & 1008 \\
\hline 9895 & 1035 \\
\hline
\end{tabular}

\begin{tabular}{|c|c|}
\hline \multicolumn{2}{|c|}{3 -core } \\
\hline Nodes & DS-RAI \\
\hline 10004 & 1292 \\
\hline 10521 & 937 \\
\hline 10567 & 1249 \\
\hline 11245 & 1839 \\
\hline 11509 & 1407 \\
\hline 11621 & 1239 \\
\hline 11770 & 1466 \\
\hline 12155 & 1402 \\
\hline 12193 & 1607 \\
\hline 12803 & 1319 \\
\hline 13364 & 2234 \\
\hline 13653 & 1760 \\
\hline 13670 & 2317 \\
\hline 13745 & 1882 \\
\hline 13878 & 2059 \\
\hline 14066 & 2159 \\
\hline 14916 & 1598 \\
\hline 14935 & 2164 \\
\hline 14923 & 2611 \\
\hline 14928 & 2509 \\
\hline 15126 & 1458 \\
\hline 15435 & 2194 \\
\hline 16515 & 2434 \\
\hline 16804 & 2532 \\
\hline 16979 & 2030 \\
\hline $1 h+D S-R A I$ \\
\hline
\end{tabular}

\begin{tabular}{|c|c|}
\hline \multicolumn{2}{|c|}{3 -core } \\
\hline Nodes & DS-RAI \\
\hline 17196 & 2063 \\
\hline 17211 & 2466 \\
\hline 17425 & 1665 \\
\hline 17444 & 2392 \\
\hline 18163 & 2403 \\
\hline 18448 & 2047 \\
\hline 18659 & 2420 \\
\hline 19693 & 3202 \\
\hline 20466 & 2947 \\
\hline 20869 & 3081 \\
\hline 21324 & 3095 \\
\hline 21676 & 2856 \\
\hline 22937 & 2880 \\
\hline 23838 & 3369 \\
\hline 24388 & 3056 \\
\hline 24569 & 3966 \\
\hline 27735 & 4186 \\
\hline 29746 & 3942 \\
\hline 30147 & 3872 \\
\hline 30809 & 4123 \\
\hline 31552 & 4846 \\
\hline 32368 & 4743 \\
\hline 35122 & 4551 \\
\hline 36371 & 4265 \\
\hline 41541 & 6327 \\
\hline
\end{tabular}

TABLE A.35. Dominating set sizes from the DS-RAI algorithm for the 3-core of DMGEO-P Facebook 100 graphs. 


\begin{tabular}{|c|c|}
\hline \multicolumn{2}{|c|}{ 4-core } \\
\hline Nodes & DS-RAI \\
\hline 760 & 147 \\
\hline 962 & 119 \\
\hline 1446 & 171 \\
\hline 1515 & 213 \\
\hline 1659 & 142 \\
\hline 2235 & 180 \\
\hline 2252 & 248 \\
\hline 2314 & 209 \\
\hline 2613 & 257 \\
\hline 2682 & 337 \\
\hline 2790 & 281 \\
\hline 2920 & 325 \\
\hline 2969 & 383 \\
\hline 2970 & 337 \\
\hline 3068 & 337 \\
\hline 3075 & 345 \\
\hline 3445 & 320 \\
\hline 3482 & 353 \\
\hline 3578 & 370 \\
\hline 3593 & 344 \\
\hline 3707 & 664 \\
\hline 3826 & 378 \\
\hline 3898 & 487 \\
\hline 4047 & 378 \\
\hline 4087 & 413 \\
\hline
\end{tabular}

\begin{tabular}{|c|c|}
\hline \multicolumn{2}{|c|}{ 4-core } \\
\hline Nodes & DS-RAI \\
\hline 4562 & 611 \\
\hline 5075 & 561 \\
\hline 5180 & 687 \\
\hline 5372 & 452 \\
\hline 6385 & 822 \\
\hline 6440 & 796 \\
\hline 6472 & 769 \\
\hline 6590 & 900 \\
\hline 6596 & 711 \\
\hline 6637 & 842 \\
\hline 6682 & 854 \\
\hline 6752 & 1228 \\
\hline 7310 & 1163 \\
\hline 7460 & 850 \\
\hline 7694 & 928 \\
\hline 7750 & 1084 \\
\hline 7755 & 859 \\
\hline 7772 & 955 \\
\hline 8069 & 795 \\
\hline 8578 & 946 \\
\hline 8600 & 875 \\
\hline 8965 & 1489 \\
\hline 8984 & 1709 \\
\hline 9414 & 1008 \\
\hline 9895 & 1035 \\
\hline
\end{tabular}

\begin{tabular}{|c|c|}
\hline \multicolumn{2}{|c|}{4 -core } \\
\hline Nodes & DS-RAI \\
\hline 10003 & 1292 \\
\hline 10521 & 937 \\
\hline 10567 & 1249 \\
\hline 11233 & 1832 \\
\hline 11509 & 1407 \\
\hline 11621 & 1239 \\
\hline 11770 & 1466 \\
\hline 12155 & 1402 \\
\hline 12192 & 1607 \\
\hline 12803 & 1319 \\
\hline 13330 & 2214 \\
\hline 13653 & 1760 \\
\hline 13623 & 2293 \\
\hline 13741 & 1879 \\
\hline 13863 & 2049 \\
\hline 14051 & 2152 \\
\hline 14916 & 1598 \\
\hline 14929 & 2161 \\
\hline 14873 & 2589 \\
\hline 14895 & 2502 \\
\hline 15126 & 1458 \\
\hline 15434 & 2194 \\
\hline 16505 & 2430 \\
\hline 16792 & 2526 \\
\hline 16979 & 2030 \\
\hline $1 h e D S-R A 1$ \\
\hline
\end{tabular}

\begin{tabular}{|c|c|}
\hline \multicolumn{2}{|c|}{ 4-core } \\
\hline Nodes & DS-RAI \\
\hline 17195 & 2063 \\
\hline 17204 & 2460 \\
\hline 17425 & 1665 \\
\hline 17443 & 2392 \\
\hline 18159 & 2400 \\
\hline 18448 & 2047 \\
\hline 18655 & 2419 \\
\hline 19667 & 3193 \\
\hline 20462 & 2945 \\
\hline 20859 & 3075 \\
\hline 21315 & 3090 \\
\hline 21673 & 2855 \\
\hline 22935 & 2878 \\
\hline 23828 & 3361 \\
\hline 24386 & 3055 \\
\hline 24532 & 3951 \\
\hline 27721 & 4181 \\
\hline 29744 & 3940 \\
\hline 30146 & 3871 \\
\hline 30804 & 4119 \\
\hline 31532 & 4835 \\
\hline 32355 & 4737 \\
\hline 35121 & 4550 \\
\hline 36371 & 4265 \\
\hline 41485 & 6303 \\
\hline
\end{tabular}

TABLE A.36. Dominating set sizes from the DS-RAI algorithm for the 4-core of DMGEO-P Facebook 100 graphs. 


\begin{tabular}{|c|c|}
\hline \multicolumn{2}{|c|}{5 -core } \\
\hline Nodes & DS-RAI \\
\hline 743 & 142 \\
\hline 960 & 119 \\
\hline 1446 & 171 \\
\hline 1508 & 212 \\
\hline 1659 & 142 \\
\hline 2235 & 180 \\
\hline 2252 & 248 \\
\hline 2314 & 209 \\
\hline 2613 & 257 \\
\hline 2678 & 336 \\
\hline 2790 & 281 \\
\hline 2919 & 324 \\
\hline 2969 & 383 \\
\hline 2970 & 337 \\
\hline 3068 & 337 \\
\hline 3075 & 345 \\
\hline 3445 & 320 \\
\hline 3482 & 353 \\
\hline 3578 & 370 \\
\hline 3593 & 344 \\
\hline 3647 & 635 \\
\hline 3826 & 378 \\
\hline 3897 & 487 \\
\hline 4047 & 378 \\
\hline 4087 & 413 \\
\hline
\end{tabular}

\begin{tabular}{|c|c|}
\hline \multicolumn{2}{|c|}{5 -core } \\
\hline Nodes & DS-RAI \\
\hline 4559 & 610 \\
\hline 5073 & 560 \\
\hline 5179 & 687 \\
\hline 5372 & 452 \\
\hline 6384 & 821 \\
\hline 6439 & 795 \\
\hline 6472 & 769 \\
\hline 6587 & 900 \\
\hline 6596 & 711 \\
\hline 6637 & 842 \\
\hline 6682 & 854 \\
\hline 6609 & 1194 \\
\hline 7265 & 1137 \\
\hline 7459 & 851 \\
\hline 7694 & 928 \\
\hline 7747 & 1082 \\
\hline 7755 & 859 \\
\hline 7771 & 955 \\
\hline 8068 & 794 \\
\hline 8578 & 946 \\
\hline 8600 & 875 \\
\hline 8910 & 1467 \\
\hline 8815 & 1640 \\
\hline 9414 & 1008 \\
\hline 9895 & 1035 \\
\hline
\end{tabular}

\begin{tabular}{|c|c|}
\hline \multicolumn{2}{|c|}{5 -core } \\
\hline Nodes & DS-RAI \\
\hline 10001 & 1290 \\
\hline 10521 & 937 \\
\hline 10565 & 1249 \\
\hline 11202 & 1820 \\
\hline 11508 & 1406 \\
\hline 11621 & 1239 \\
\hline 11766 & 1464 \\
\hline 12154 & 1401 \\
\hline 12190 & 1606 \\
\hline 12803 & 1319 \\
\hline 13244 & 2175 \\
\hline 13650 & 1758 \\
\hline 13488 & 2253 \\
\hline 13731 & 1872 \\
\hline 13819 & 2027 \\
\hline 14029 & 2144 \\
\hline 14916 & 1598 \\
\hline 14904 & 2155 \\
\hline 14732 & 2536 \\
\hline 14782 & 2450 \\
\hline 15126 & 1458 \\
\hline 15426 & 2192 \\
\hline 16475 & 2424 \\
\hline 16756 & 2516 \\
\hline 16978 & 2030 \\
\hline $1 h+D S-R A I$ \\
\hline
\end{tabular}

\begin{tabular}{|c|c|}
\hline \multicolumn{2}{|c|}{5 -core } \\
\hline Nodes & DS-RAI \\
\hline 17194 & 2063 \\
\hline 17179 & 2454 \\
\hline 17425 & 1665 \\
\hline 17439 & 2390 \\
\hline 18156 & 2399 \\
\hline 18447 & 2046 \\
\hline 18653 & 2419 \\
\hline 19600 & 3170 \\
\hline 20451 & 2936 \\
\hline 20834 & 3058 \\
\hline 21286 & 3079 \\
\hline 21652 & 2846 \\
\hline 22929 & 2875 \\
\hline 23807 & 3355 \\
\hline 24382 & 3053 \\
\hline 24432 & 3910 \\
\hline 27677 & 4160 \\
\hline 29736 & 3935 \\
\hline 30140 & 3866 \\
\hline 30793 & 4112 \\
\hline 31460 & 4815 \\
\hline 32296 & 4718 \\
\hline 35118 & 4549 \\
\hline 36369 & 4263 \\
\hline 41305 & 6251 \\
\hline
\end{tabular}

TABLE A.37. Dominating set sizes from the DS-RAI algorithm for the 5-core of DMGEO-P Facebook 100 graphs. 



\section{APPENDIX B}

\section{Program Code}

The following two sections describe the code used during the research of this thesis. The first section provides Java code that was used along with the JUNG 2.0.1 framework to generate visual MGEO-P and DMGEO-P samples. The second section is the $\mathrm{C}++$ code used injunction with the Stanford Network Analysis Platform (SNAP).

The MGEO-P simulations were generated with a Quad Core i7-4700MQ 2.40 GHz $16 \mathrm{~GB}$ laptop. The computation of the domination number of the data sets were done using RAMLab equipment. The hardware used during DMGEO-P experiments, simulations and domination, was a 12-core (2x 6core) Xeon X5690 3.46 GHz with 96 GB RAM workstation.

An online simulator for the $\mathrm{G}(n, p), \mathrm{G}(n, r)$ and $\operatorname{MGEO}-\mathrm{P}(n, m, \alpha, \beta, p)$ models were created for presentation purposes.

Simulator is located at http://www.math.ryerson.ca/people/mlozier/.

\section{B.1. JUNG Code (Java)}

createGUI.java

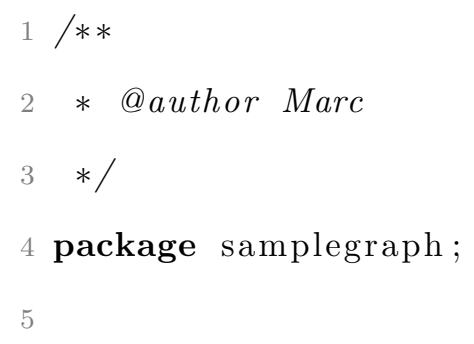




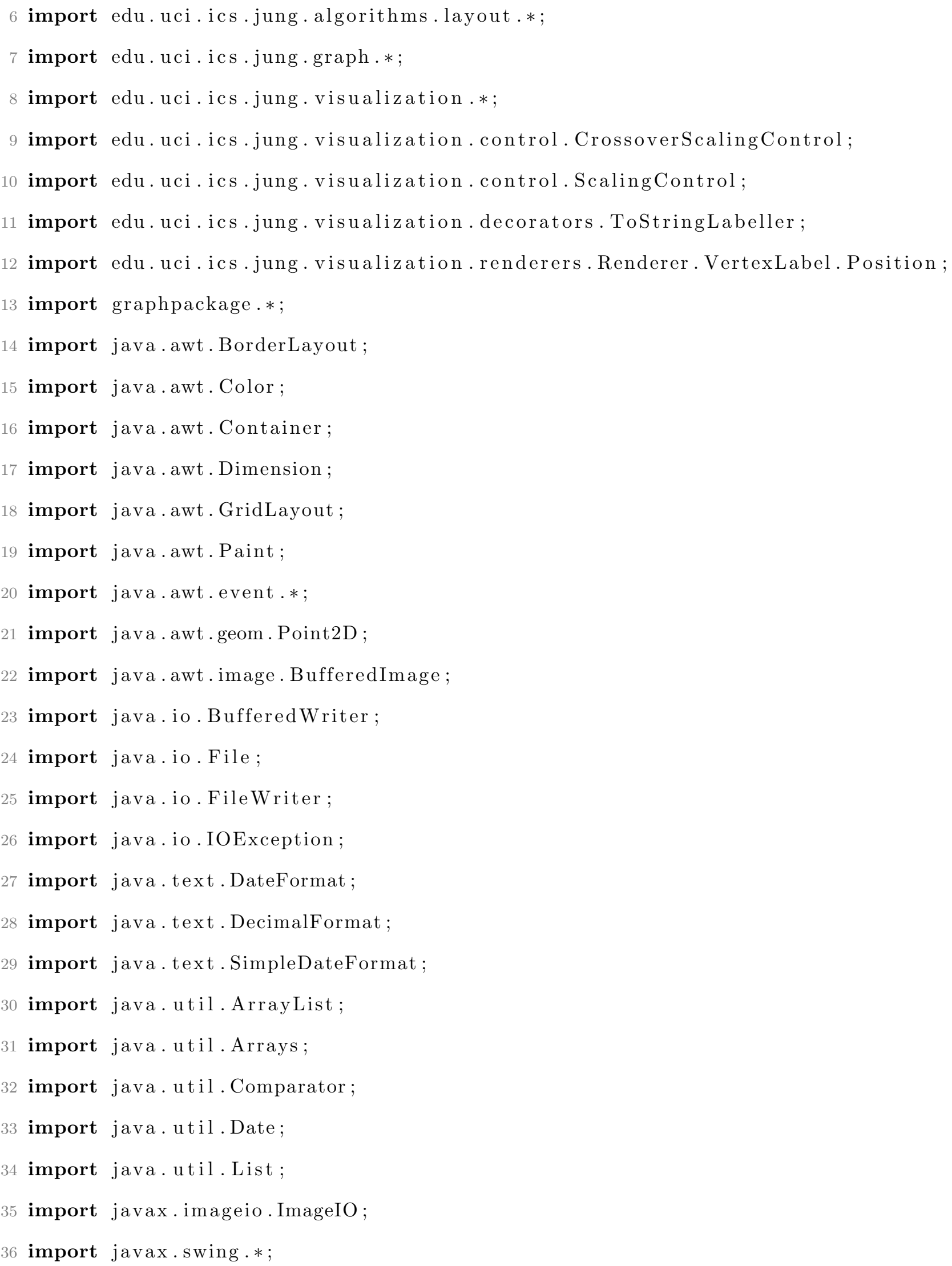


37 import org.apache.commons. collections 15 . Transformer;

38

39 public class createGUI extends JApplet implements ActionListener, MouseWheelListener \{

$40 \quad / /$ constants for action commands

41 protected final static int GNP_GRAPH $=1$;

42 protected final static int GNR_GRAPH $=2$; 
private static final JTextField txtNodes $2=$ new JTextField("10",3); private static final JTextField txtP = new JTextField ("0.5",3); private static final JTextField txtR = new JTextField ("0.1",3); private static final JTextField txtK = new JTextField ("5",3); private static final JTextField txtN = new JTextField("769",3); // mgeop private static final JTextField txtM = new JTextField("4",3); // mgeop private static final JTextField txtAlpha = new JTextField("0.17",3); // mgeop private static final JTextField txtBeta = new JTextField $(" 0.27 ", 3) ; / /$ mgeop private static final JTextField txtP_MGEOP = new JTextField ("1",3);// mgeop private static final JTextField txtE_MGEOP = new JTextField ("16656",8); // mgeop

public static int graphType = GNP_GRAPH;

private static JComponent graph;

private static BufferedImage image;

private static boolean blnLabel = true;

private static boolean blnShowVisual = true;

private static boolean blnNewWindow = true;

private static UndirectedSparseGraph $<$ Node, String $>$ g $=$ null;

private static List $<$ Node $>$ dominatingSet $=$ new ArrayList $<>()$;

public createGUI ()\{

//constructor

\}

public void createGraphWithDS() \{ generateVisualGraph (true); graphFrame.pack ();

\} 


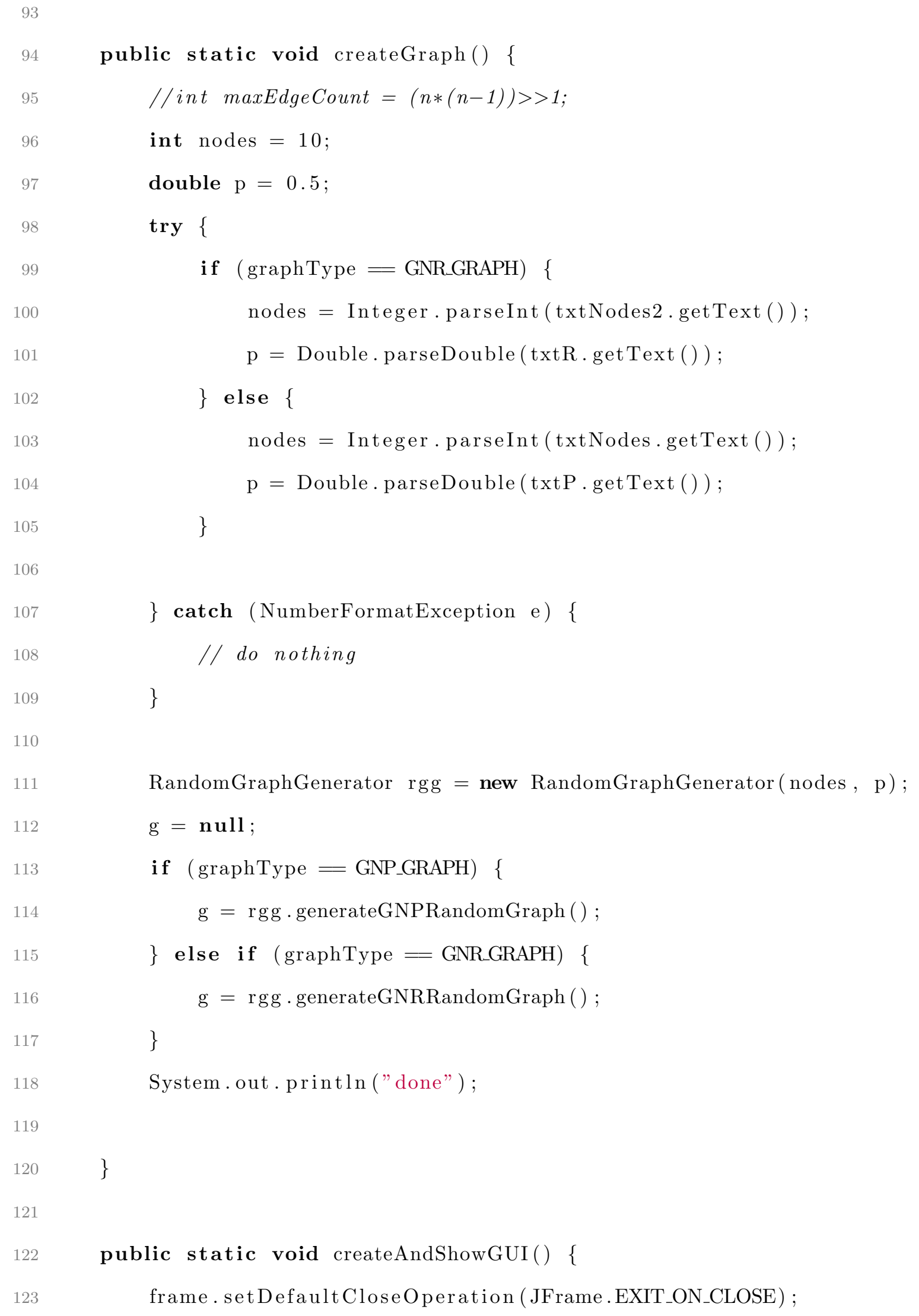


createGUI maingui $=$ new createGUI () ;

maingui.createTabs ();

//Display the window.

frame.pack ();

frame.setLocationRelativeTo(null); //center it frame. setVisible (true);

\}

public static void generateVisualGraph(boolean showDS) \{

Container contentPane $=$ graphFrame getContentPane () ;

if $($ graph $!=$ null $)\{$

contentPane.remove (graph);

\}

Layout $<$ Node, String $>$ layout $=$ null

try \{

layout $=$ new CircleLayout $<>(\mathrm{g}) ;$

\} $\operatorname{catch}($ Exception e) \{

// do nothing

return;

\}

layout.setSize (new Dimension(800, 800));

BasicVisualizationServer $<$ Node, String $>\mathrm{vv}=$ new

BasicVisualizationServer $<>$ (layout);

vv. setPreferredSize (new Dimension(800, 800));

ScalingControl scaler = new CrossoverScalingControl();

scaler.scale (vv, $1 / 1.1 \mathrm{f}$, vv.getCenter ()); 


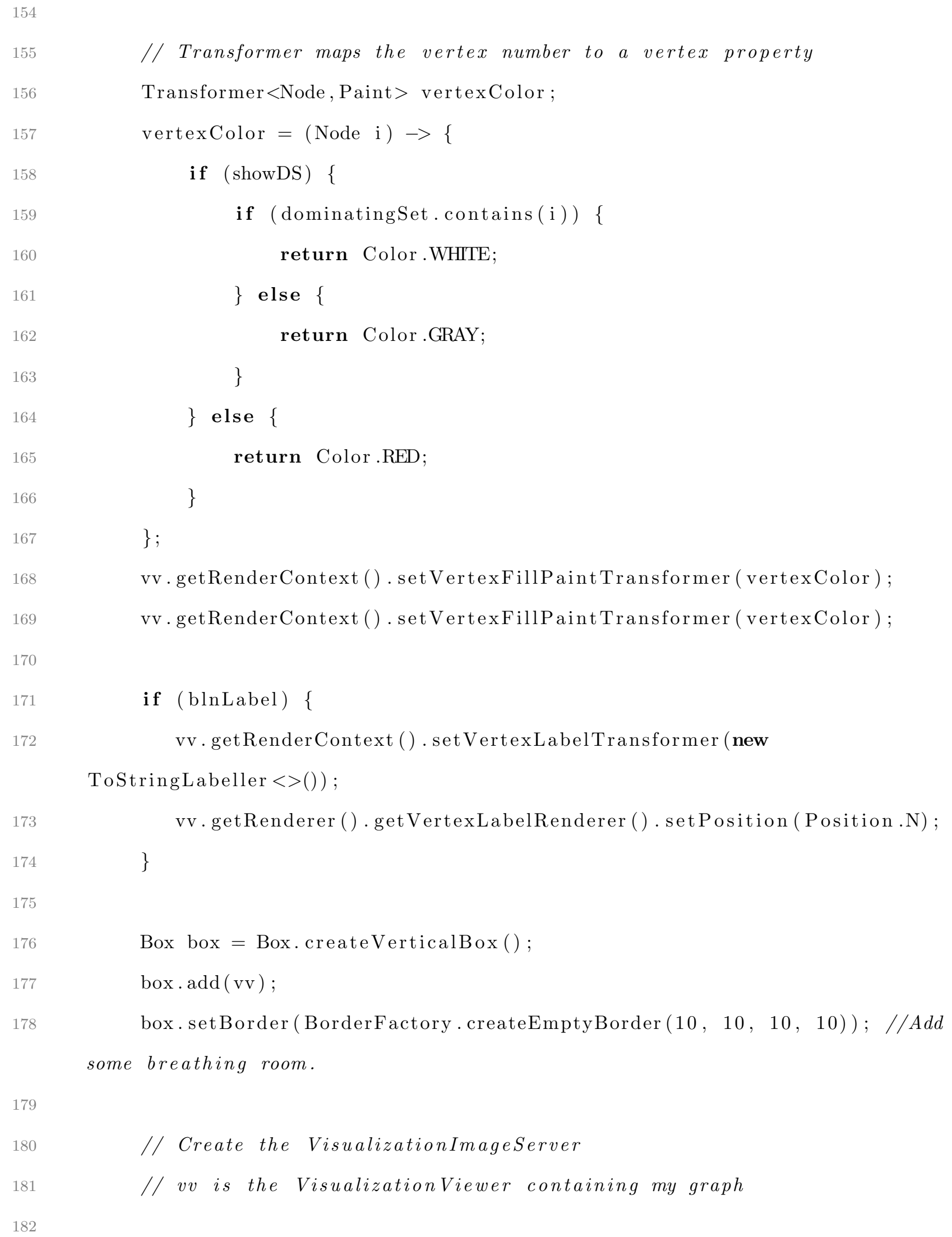

// Transformer maps the vertex number to a vertex property Transformer $<$ Node, Paint $>$ vertexColor ; vertexColor $=($ Node $\mathrm{i}) \rightarrow\{$ if $($ showDS $)\{$ if (dominatingSet. contains (i)) \{ return Color.WHITE;

\} else \{ return Color.GRAY;

\}

\} else \{ return Color. $\mathrm{RED}$;

\}

\}

vv.getRenderContext (). setVertexFillPaintTransformer (vertexColor); vv.getRenderContext (). setVertexFillPaintTransformer (vertexColor);

if $(\mathrm{b} \ln L \mathrm{abel})\{$ vv.getRenderContext (). setVertexLabelTransformer (new ToStringLabeller $<>())$; vv.getRenderer ( ) . getVertexLabelRenderer (). setPosition ( Position.N); \}

Box box = Box. createVerticalBox () ;

box. $\operatorname{add}(\mathrm{vv})$;

box. setBorder (BorderFactory . createEmptyBorder $(10,10,10,10)$; //Add some breathing room.

// Create the VisualizationImageServer

// vv is the VisualizationViewer containing my graph 
VisualizationImageServer $<$ Node, String $>$ vis $=$ new

VisualizationImageServer $<>$ (vv.getGraphLayout (), vv getGraphLayout () . getSize ());

184

185

186

187

188

189

190

191

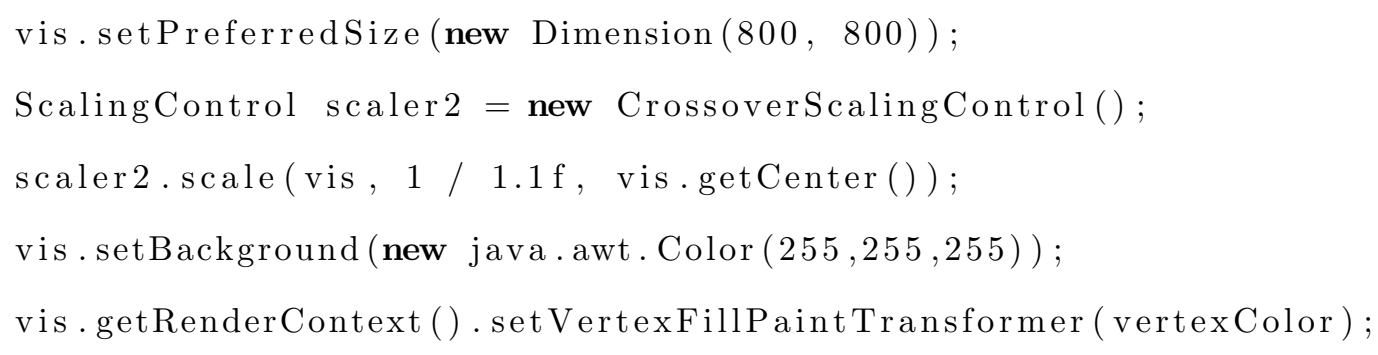




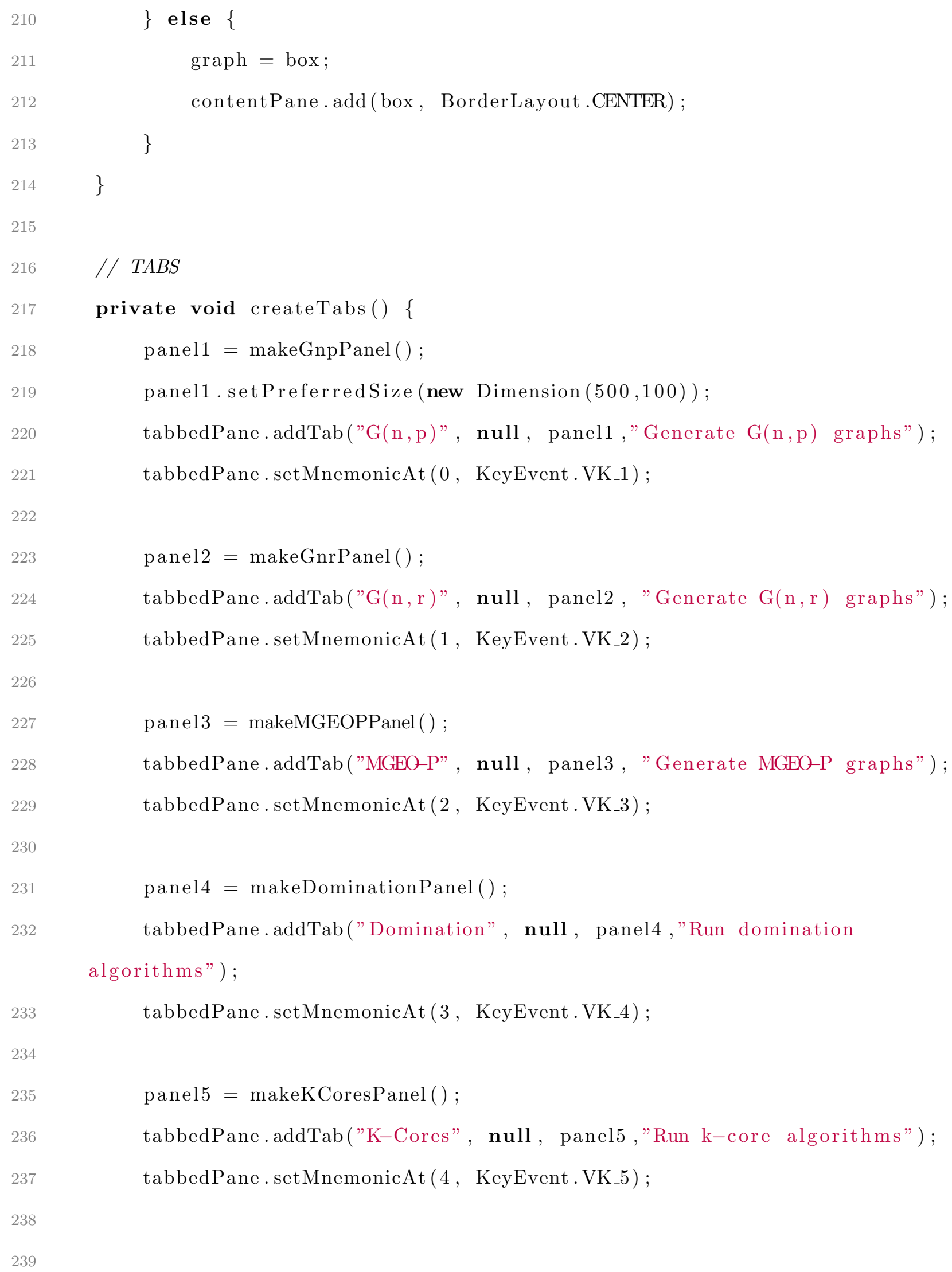




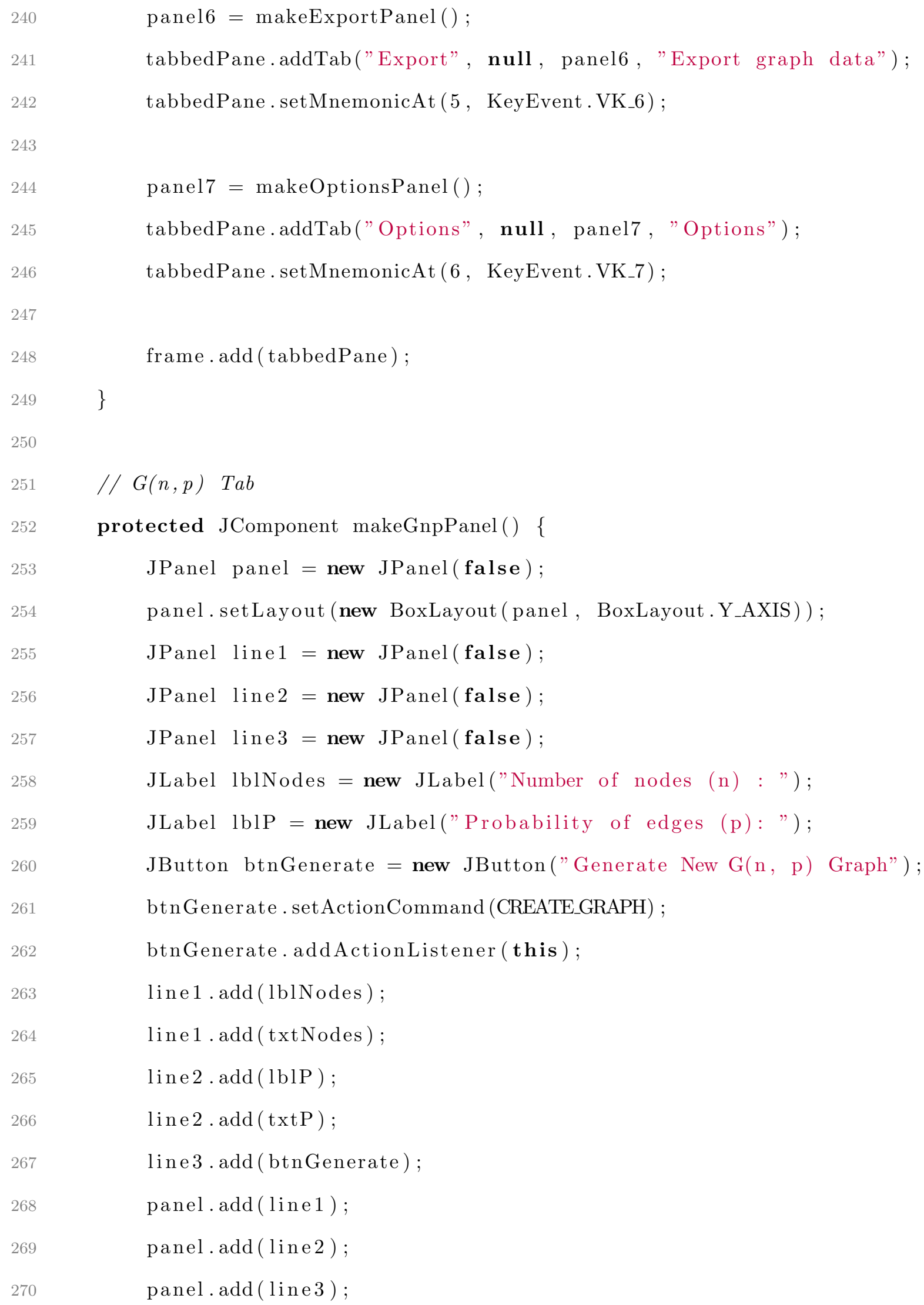

panel6 = makeExportPanel ();

tabbedPane.addTab("Export", null, panel6, "Export graph data"); tabbedPane.setMnemonicAt (5, KeyEvent.VK_6);

panel7 = makeOptionsPanel ()$;$ tabbedPane.addTab("Options", null, panel7, "Options"); tabbedPane.setMnemonicAt (6, KeyEvent.VK_7);

frame .add (tabbedPane);

\}

$/ / G(n, p) \quad T a b$

protected JComponent makeGnpPanel() \{

JPanel panel $=$ new $\operatorname{JPanel}($ false $)$;

panel.setLayout(new BoxLayout(panel, BoxLayout.Y_AXIS));

JPanel line 1 = new $\operatorname{JPanel}($ false);

JPanel line 2 = new JPanel(false);

JPanel line $3=$ new JPanel(false);

JLabel lblNodes $=$ new JLabel ("Number of nodes (n) : ");

JLabel lblP $=$ new JLabel("Probability of edges (p): ");

JButton btnGenerate = new JButton("Generate New G(n, p) Graph");

btnGenerate . setActionCommand (CREATE_GRAPH) ;

btnGenerate . add ActionListener (this );

line 1 . add (lblNodes);

line $1 . \operatorname{add}($ txtNodes $)$;

line 2 . add (lblP);

line 2 . add $(\operatorname{txtP})$;

line 3 . add (btnGenerate);

panel.add ( line1);

panel.add ( line 2);

panel.add ( line 3$)$; 


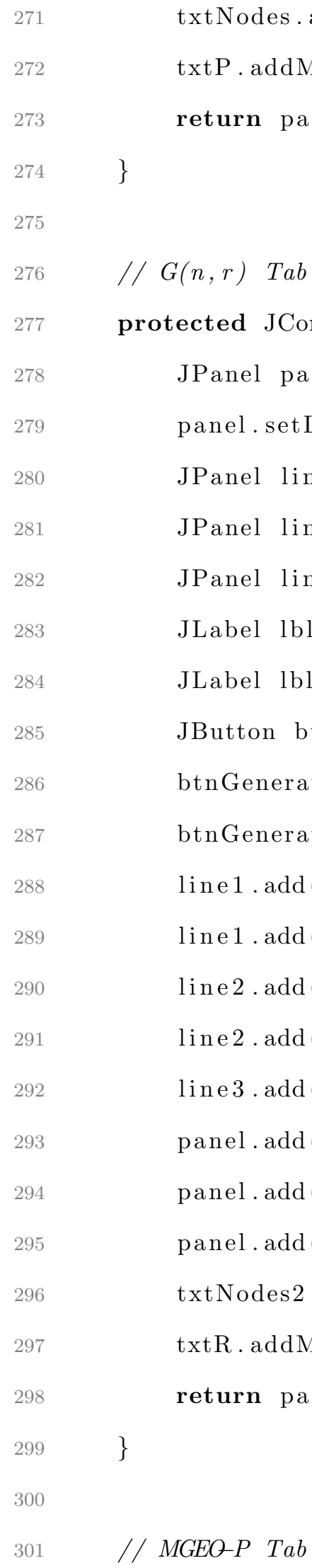

// MGEO-P Tab 
protected JComponent makeMGEOPPanel() \{

JPanel panel $=$ new $\operatorname{JPanel}($ false $)$;

panel.setLayout(new BoxLayout(panel, BoxLayout.Y_AXIS));

JPanel line1 = new JPanel(false);

JPanel line 2 = new JPanel(false);

JPanel line $3=$ new JPanel(false);

JPanel line $4=$ new $\operatorname{JPanel}($ false $)$;

JPanel line $5=$ new $\operatorname{JPanel}($ false);

JPanel line6 = new JPanel(false);

JPanel line $7=$ new $\operatorname{JPanel}($ false $)$;

JPanel line $8=$ new $\mathrm{JPanel}($ false);

JLabel 1 blN = new JLabel ("Number of vertices (n) : ");

JLabel lblM = new JLabel("Dimension of metric space (m) : ");

JLabel lblAlpha $=$ new JLabel ("Attachment strength parameter (alpha) : $")$;

JLabel lblBeta $=$ new JLabel("Density parameter (beta) : ");

JLabel lblP = new JLabel("Connection probability (p) : ");

JLabel lble = new JLabel ("Set Edges (e) : ");

JButton btnMGEOP = new JButton("Generate New MGEO-P(n, m, alpha, beta,

p) Graph");

JButton btnMGEOPINV = new JButton ("INV-MGEO-P");

btnMGEOP . set ActionCommand (MGEOP_GRAPH) ;

btnMGEOP . add ActionListener ( this );

btnMGEOPINV . setActionCommand ("INV-MGEO-P" ) ;

btnMGEOPINV . add ActionListener ( this );

line $1 \cdot \operatorname{add}(\mathrm{lblN})$;

line $1 . \operatorname{add}(\operatorname{txtN})$;

line 2 . add (lblM);

line $2 \cdot \operatorname{add}(\operatorname{txtM})$; 
line 3 . add (lblAlpha);

line 3 . add (txtAlpha);

line 4 . add (lblBeta);

line 4 . add (txtBeta);

line 5 . add (lblP);

line 5 . add (txtP_MGEOP) ;

line $6 \cdot \operatorname{add}(\mathrm{lblE})$;

line 6 . add (txtE_MGEOP);

line 7 . add (btnMGEOP) ;

line 8 . add (btnMGEOPINV);

panel.add ( line 1);

panel.add (line 2);

panel.add (line 3$)$;

panel.add (line 4);

panel.add ( line 5);

panel.add ( line6);

panel.add (line 7$)$;

panel.add ( line 8);

txtN . addMouseWheelListener (this );

txtM . addMouseWheelListener (this );

txtAlpha . addMouseWheelListener (this);

txtBeta . addMouseWheelListener (this);

txtP_MGEOP . addMouseWheelListener ( this ) ;

return panel;

\}

// Domination Tab

protected JComponent makeDominationPanel() \{

JPanel panel $=$ new $\operatorname{JPanel}($ false $)$;

panel.setLayout(new BoxLayout(panel, BoxLayout.Y_AXIS)); 
final JButton btnDegreeDistribution = new JButton("Degree Distribution (Histogram)");

388

btnDegreeDistribution . setActionCommand (GRAPHDEGREEDIST) ;

btnDegreeDistribution . addActionListener (this) ;

final JButton btnDiameter = new JButton("Diameter");

btnDiameter . setActionCommand (GRAPH DIAMETER);

btnDiameter . addActionListener (this );

final JButton btnDSRAI1 = new JButton("DS-RAI (PHASE 1)"); btnDSRAI1 . setActionCommand (GRAPH_DSRAIPHASE1) ; btnDSRAI1 add ActionListener (this );

final JButton btnDSRAI2 = new JButton("DS-RAI (PHASE 2)"); btnDSRAI2 . setActionCommand (GRAPH_DSRAIPHASE1); btnDSRAI2 . add ActionListener ( $\mathbf{t h}$ is );

final JButton btnDSRAI3 = new JButton("DS-RAI (PHASE 3)"); btnDSRAI3 . setActionCommand (GRAPH_DSRAIPHASE3) ; btnDSRAI3 . addActionListener (this );

final JButton btnDSDC = new JButton("DS-DC"); btnDSDC . set Action Command (GRAPHDSDC) ; btnDSDC . addActionListener (this);

final JButton btnBurning = new JButton("Burning"); btnBurning · set ActionCommand ("BURNING") ; btnBurning . addActionListener (this);

panel .add (btnDegreeDistribution ); panel.add (btnDiameter); 


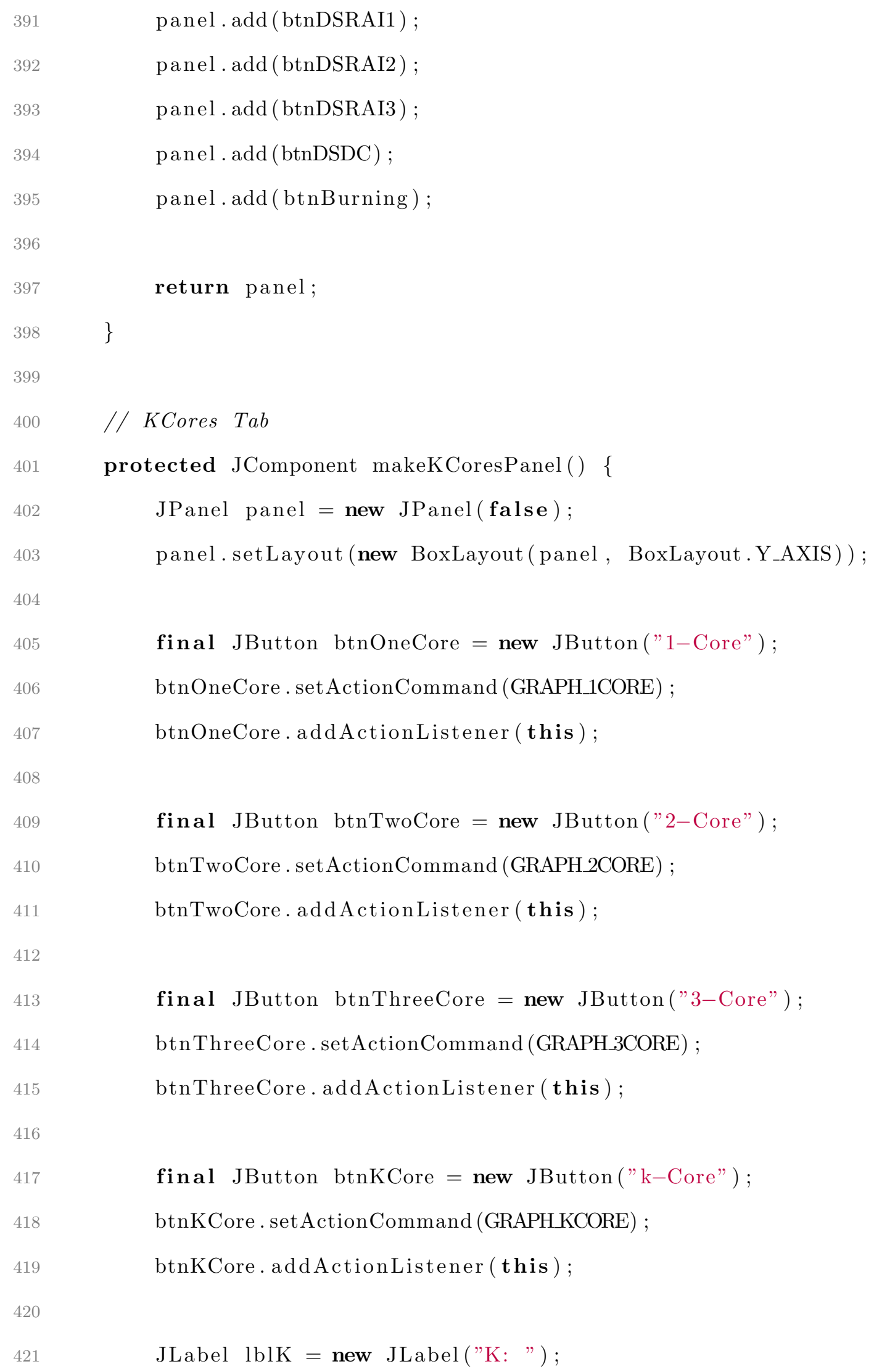

panel. add (btnDSRAI1);

panel .add (btnDSRAI2);

panel. add (btnDSRAI3);

panel . add (btnDSDC) ;

panel.add (btnBurning);

return panel;

\}

// KCores Tab

protected JComponent makeKCoresPanel() \{

JPanel panel $=$ new $\operatorname{JPanel}($ false $)$;

panel.setLayout (new BoxLayout (panel, BoxLayout.Y_AXIS)) ;

final JButton btnOneCore = new JButton("1-Core");

btnOneCore . set ActionCommand (GRAPH_1CORE) ;

btnOneCore. add ActionListener (this);

final JButton btnTwoCore = new JButton("2-Core");

btnTwoCore set Action Command (GRAPH 2CORE) ;

btnTwoCore. add ActionListener ( this );

final JButton btnThreeCore = new JButton("3-Core");

btnThreeCore . setActionCommand (GRAPH_3CORE) ;

btnThreeCore. addActionListener ( this ) ;

final JButton btnKCore = new JButton ("k-Core");

btnKCore . setActionCommand (GRAPH KCORE);

btnKCore. addActionListener (this);

JLabel $1 \mathrm{blK}=$ new JLabel ("K: ") ; 


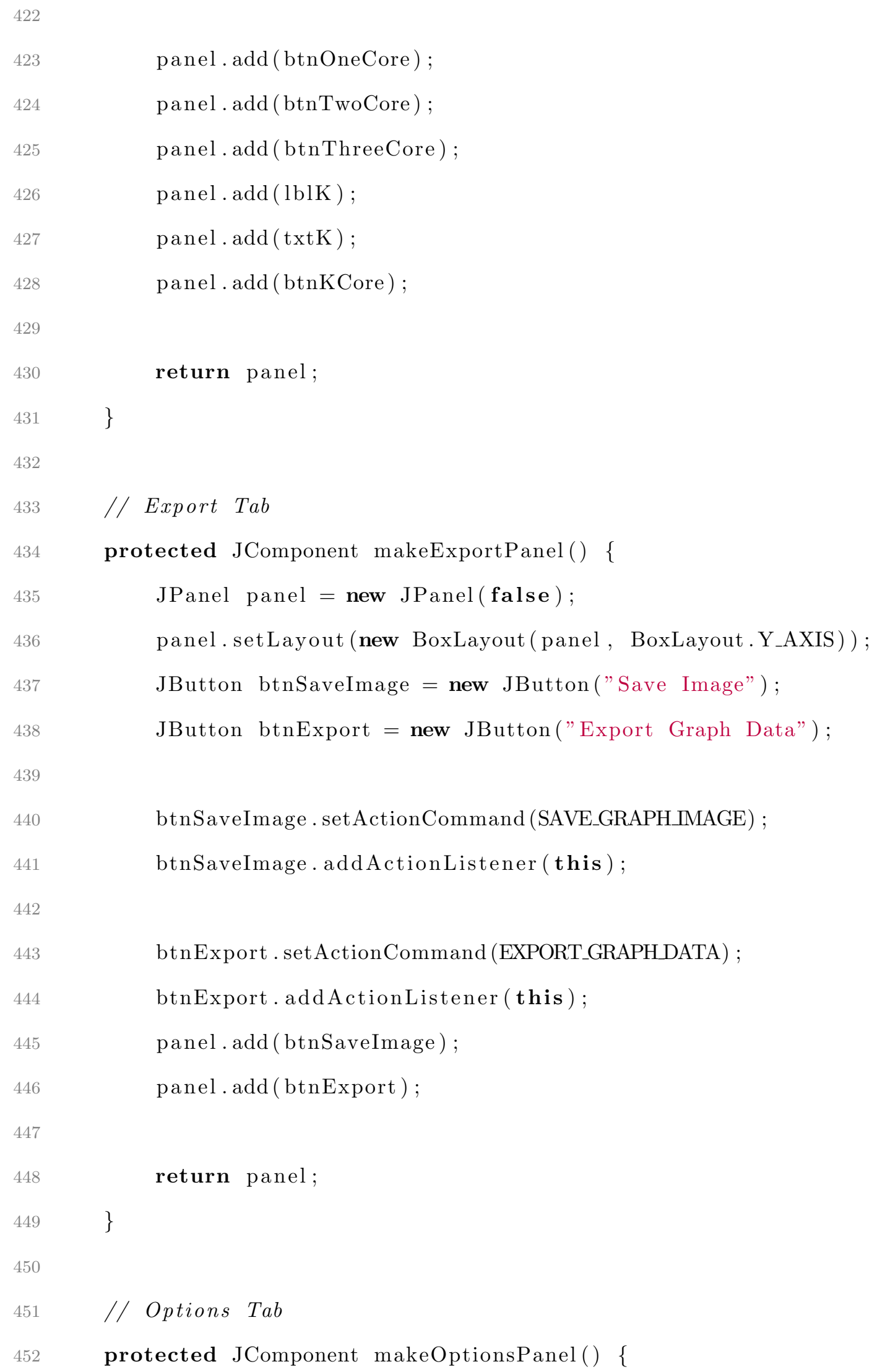




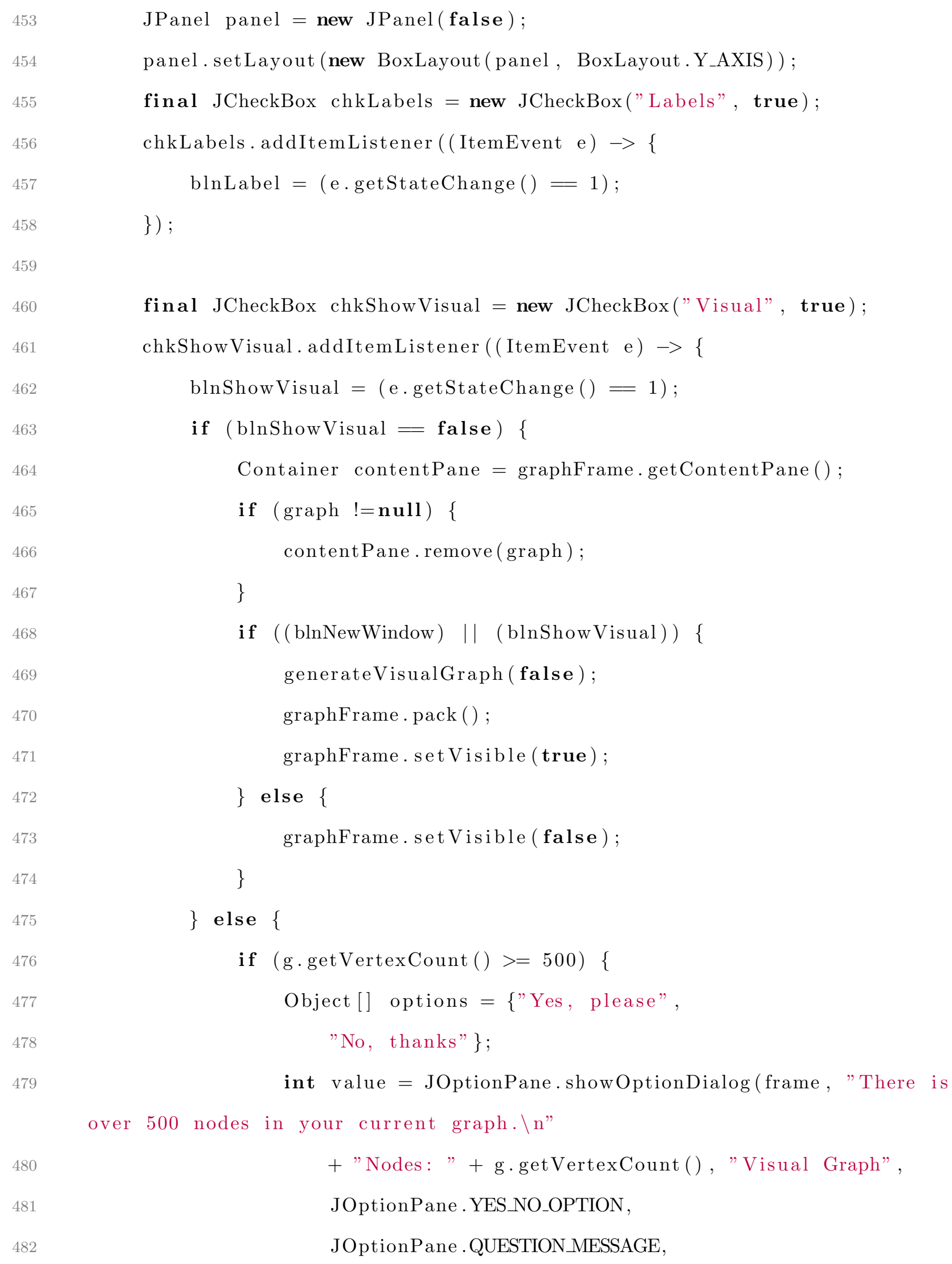

JPanel panel $=$ new $\operatorname{JPanel}($ false $)$; panel.setLayout(new BoxLayout (panel, BoxLayout.Y_AXIS)) ;

final JCheckBox chkLabels = new JCheckBox("Labels", true); chkLabels . addItemListener ( (ItemEvent e $) \rightarrow\{$ blnLabel $=($ e.getStateChange ()$=1)$; \});

final JCheckBox chkShowVisual = new JCheckBox("Visual", true); chkShowVisual addItemListener ( (ItemEvent e $) \rightarrow\{$ blnShowVisual $=($ e $\cdot \operatorname{get}$ StateChange ()$=1)$; if (blnShowVisual = false $)\{$ Container contentPane $=$ graphFrame $\cdot$ getContentPane () ; if $(\operatorname{graph} \quad !=\mathbf{n u l l}) \quad\{$ contentPane.remove (graph); \} if $(($ blnNewWindow $) \|($ blnShowVisual $))\{$ generateVisualGraph (false); graphFrame $\cdot \operatorname{pack}()$; graphFrame. setVisible (true); \}else \{ graphFrame. setVisible (false); \} \} else \{ if $(\mathrm{g} \cdot \operatorname{get} V$ ertexCount ()$>=500)\{$ Object [] options $=\{$ Yes, please", "No, thanks" \};

int value $=$ JOptionPane.showOptionDialog (frame, "There is over 500 nodes in your current graph.\n" + "Nodes: " + g.getVertexCount (), "Visual Graph", JOptionPane . YES_NO_OPTION, JOptionPane .QUESTION_MESSAGE, 


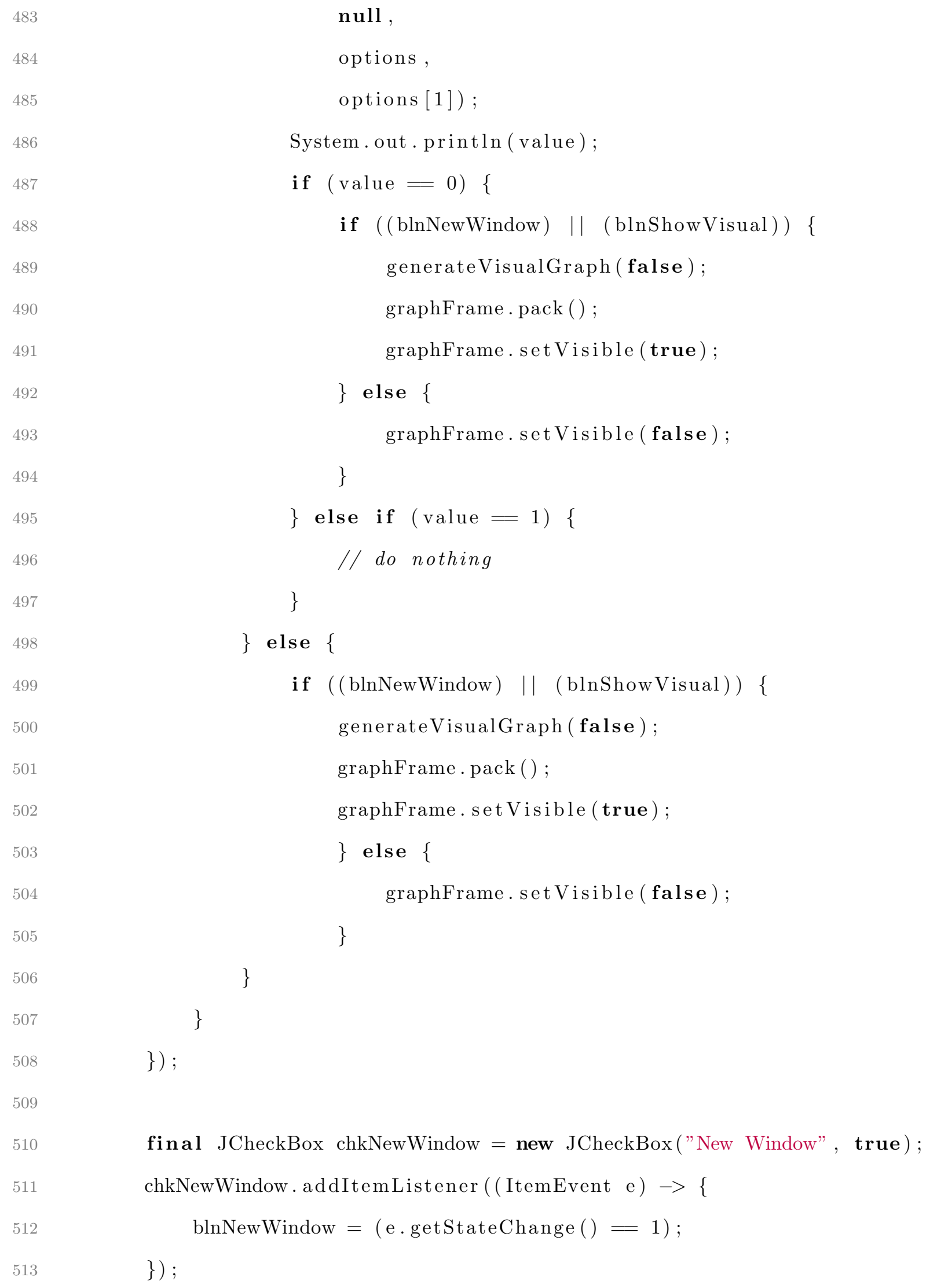

null, options, options [1]); System.out.println (value); if $($ value $=0)\{$ if $(($ blnNewWindow $) \|($ blnShowVisual $))\{$ generateVisualGraph (false); graphFrame $\cdot \operatorname{pack}()$; graphFrame. setVisible (true);

\} else \{ graphFrame. set Visible (false); 
panel.add (chkLabels);

panel.add (chkShowVisual);

panel . add (chkNewWindow);

return panel;

\}

protected static JComponent makeTextPanel(String text) \{

JPanel panel $=$ new $\operatorname{JPanel}($ false $)$;

JLabel filler = new JLabel(text);

filler . setHorizontalAlignment (JLabel.CENTER);

panel.setLayout (new GridLayout $(1,1))$;

panel.add (filler);

return panel;

\}

protected JComponent createButtonPane() \{

JPanel btmPanel = new JPanel();

JButton button = new JButton("Generate New G(n, p) Graph");

JButton button 2 = new JButton("Generate New G(n, r) Graph");

JButton btnMGEOP = new JButton ("MGEO-P");

JButton btnSaveImage = new JButton("Save Image");

JButton btnExport $=$ new JButton("Export Graph Data");

JLabel lblNodes = new JLabel("n: ");

JLabel $1 \mathrm{blP}=$ new JLabel("P/R: ");

button . setActionCommand (CREATE_GRAPH);

button . addActionListener (this); 


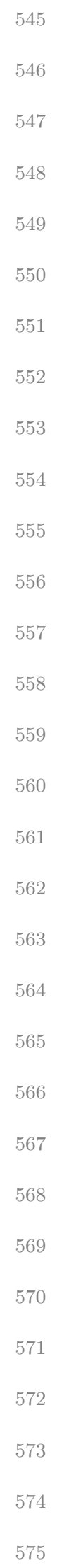


btmPanel.setLayout (new BoxLayout (btmPanel, BoxLayout.Y_AXIS)) ; btmPanel . add (pane);

btmPanel .add ( pane2);

btmPanel .add ( pane3);

btmPanel .add ( pane4);

btmPanel.add ( pane5);

return btmPanel;

\}

@ Override

public void actionPerformed(ActionEvent e) \{

String $\operatorname{command}=$ e.getActionCommand () ;

int nodes, m;

double p, alpha, beta;

if (null != command) //Handle the New window button. //Handle the New window button.

switch (command) \{

case CREATE_GRAPH:

graphType = GNP_GRAPH;

createGraph ();

if $(\mathrm{b} \ln$ ShowVisual $)\{$

generateVisualGraph ( false );

graphFrame $\cdot \operatorname{pack}()$;

graphFrame. setVisible (true);

\}

graphFrame. setTitle ("G(n="+txtNodes $\operatorname{getText}()+", p="+\operatorname{txt} P$.

$\operatorname{getText}()+")-"+\operatorname{g} \cdot \operatorname{getVertexCount}()+" \operatorname{nodes}-"+\operatorname{g} \cdot \operatorname{get} \operatorname{EdgeCount}()+"$ edges") ;

break ; 


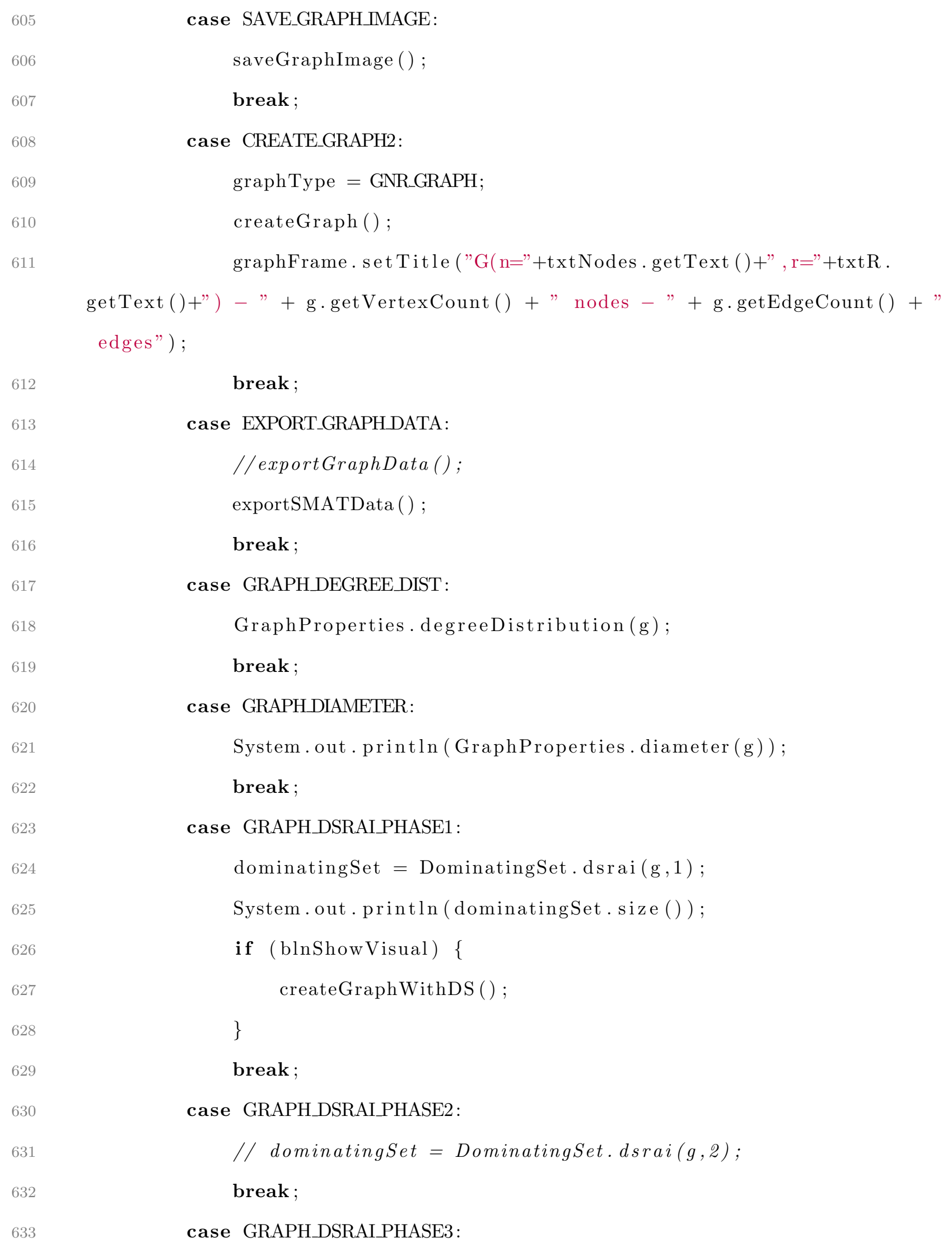

\section{case SAVE_GRAPH_IMAGE:}

saveGraphImage ();

break;

case CREATE_GRAPH2:

graphType = GNR_GRAPH;

createGraph ();

graphFrame. setTitle ("G(n="+txtNodes $\cdot \operatorname{getText}()+", r="+\operatorname{txtR}$.

break;

case EXPORT_GRAPH_DATA:

//exportGraphData();

exportSMATData () ;

break;

case GRAPHDEGREEDIST:

GraphProperties. degreeDistribution (g);

break;

case GRAPHDIAMETER:

System . out . println (GraphProperties.diameter (g));

break;

case GRAPH_DSRAIPHASE1:

dominatingSet $=$ DominatingSet.dsrai $(g, 1)$;

System . out . println (dominatingSet.size ());

if (blnShowVisual) \{

createGraphWithDS ( ) ;

\}

break;

case GRAPHDSRAIPHASE2 :

$/ /$ dominatingSet $=$ DominatingSet.dsrai $(g, 2)$;

break;

case GRAPH_DSRAI_PHASE3: 


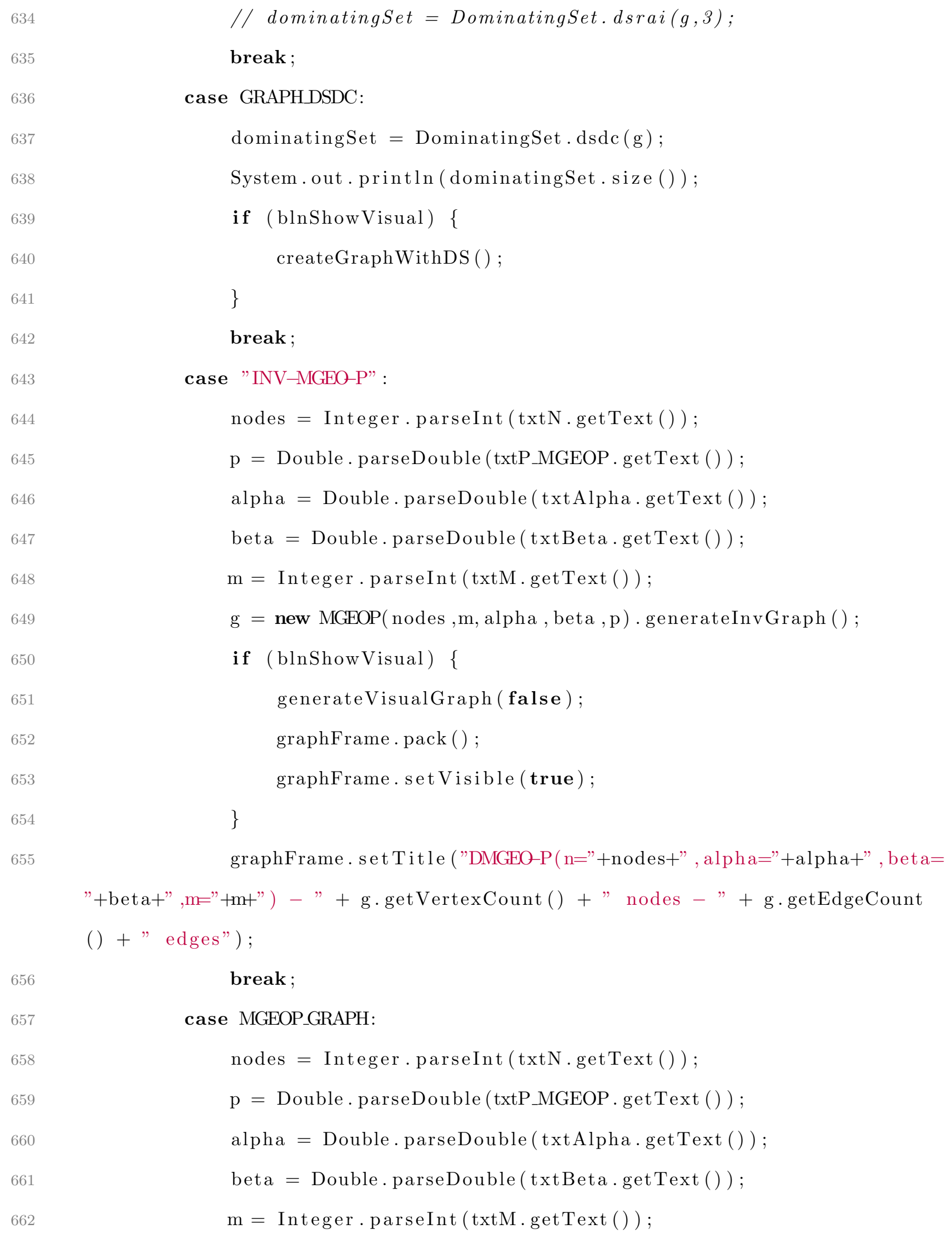

// dominatingSet = DominatingSet.dsrai $(g, 3)$;

break ;

case GRAPHDSDC:

dominatingSet $=$ DominatingSet.dsdc $(\mathrm{g})$;

System . out . println (dominatingSet. size ());

if (blnShowVisual) \{

createGraphWithDS ( ) ;

\}

break ;

case "INV-MGEO-P" :

nodes $=$ Integer $\cdot$ parseInt $(\operatorname{txtN} \cdot \operatorname{getText}())$;

$\mathrm{p}=$ Double $\cdot$ parseDouble (txtP_MGEOP.getText $($ ) ) ;

alpha $=$ Double $\cdot$ parseDouble $($ txtAlpha.getText ()$)$;

beta $=$ Double $\cdot$ parseDouble $($ txtBeta.getText ()$)$;

$\mathrm{m}=$ Integer $\cdot$ parseInt $(\operatorname{txtM} \cdot \operatorname{getText}())$

$\mathrm{g}=$ new MGEOP(nodes, $\mathrm{m}$, alpha, beta, $\mathrm{p}) \cdot$ generateInvGraph ( ) ;

if $(\mathrm{b} \ln \mathrm{Show}$ Visual $)\{$

generateVisualGraph ( false );

graphFrame $\cdot \operatorname{pack}()$;

graphFrame. setVisible (true);

\}

graphFrame. setTitle ("DMGEO-P ( n="+nodes+" , alpha="+alpha+" , beta= "+beta $+", m="+m+")-"+g \cdot g e t V e r t e x C o u n t()+"$ nodes $-"+$ g.getEdgeCount ()$+" \operatorname{edges} ") ;$

656

9

60

1

break ;

case MGEOP_GRAPH:

nodes $=$ Integer $\cdot$ parseInt $(\operatorname{txtN} \cdot \operatorname{get} T \operatorname{ext}())$;

$\mathrm{p}=$ Double $\cdot$ parseDouble (txtP_MGEOP.getText ()$)$;

alpha $=$ Double $\cdot$ parseDouble $($ txtAlpha.getText ()$)$;

beta $=$ Double $\cdot$ parseDouble $($ txtBeta.getText ()$)$;

$\mathrm{m}=$ Integer $\cdot$ parseInt $(\operatorname{txtM} \cdot \operatorname{get} T \operatorname{ext}()) ;$ 


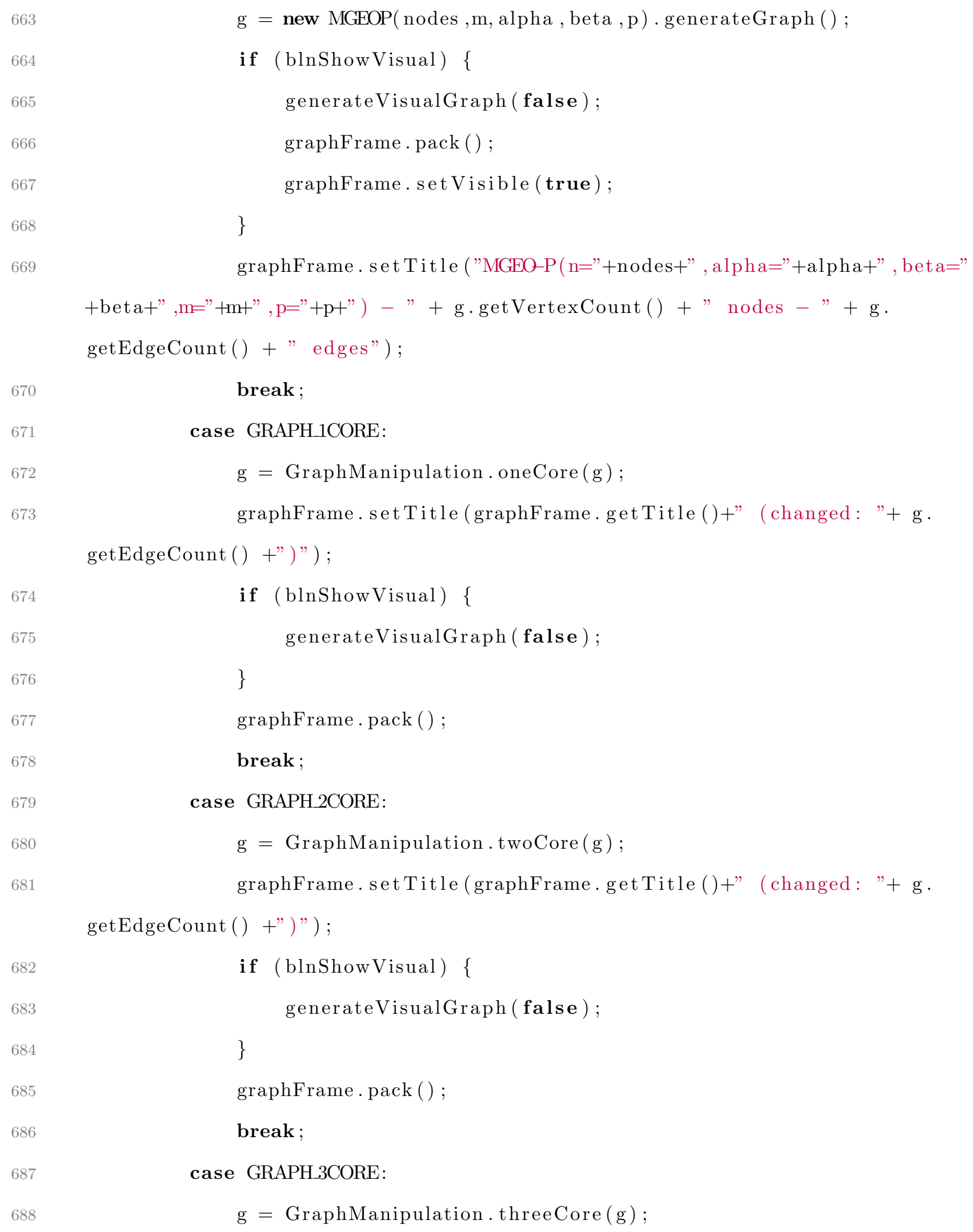

$\mathrm{g}=$ new $\mathrm{MGEOP}($ nodes, $\mathrm{m}$, alpha, beta, $\mathrm{p}) \cdot$ generateGraph () ;

if $(\mathrm{b} \ln$ ShowVisual) \{ generateVisualGraph ( false ); graphFrame $\cdot \operatorname{pack}()$; graphFrame. setVisible (true);

\} graphFrame. set Title ("MGEO-P (n="+nodes+" , alpha="+alpha+" , beta=" + beta $+", m="+m+", p="+p+")-"+g \cdot \operatorname{getVertex} \operatorname{count}()+" \operatorname{nodes}-"+\mathrm{g}$. getEdgeCount () + "edges");

break ;

case GRAPH_1CORE:

$\mathrm{g}=$ GraphManipulation . oneCore $(\mathrm{g})$;

graphFrame. setTitle (graphFrame. getTitle ()+" (changed: "+ g. getEdgeCount ( ) +")");

if $(\mathrm{b} \ln \mathrm{Show}$ Visual) \{

generateVisualGraph ( false );

\}

graphFrame $\cdot \operatorname{pack}()$;

break ;

case GRAPH_CORE:

$\mathrm{g}=$ GraphManipulation . twoCore $(\mathrm{g})$;

graphFrame. setTitle (graphFrame. getTitle ()$+"$ (changed: "+ g. getEdgeCount ()+")");

if $(\mathrm{b} \ln$ ShowVisual) \{ generateVisualGraph (false);

\}

graphFrame.pack ();

break ;

case GRAPH_3CORE:

$\mathrm{g}=$ GraphManipulation . threeCore $(\mathrm{g})$; 
graphFrame. setTitle (graphFrame.getTitle ()$+" \quad$ ( changed: "+ g. getEdgeCount ()+")");

if (blnShowVisual) \{

generateVisualGraph (false);

\}

graphFrame.pack ();

break ;

case GRAPH KCORE:

$\mathrm{g}=$ GraphManipulation.kCore (g, Integer.parseInt (txtK.getText ()

)) ;

graphFrame. setTitle (graphFrame.getTitle()+" (changed: "+ g.

getEdgeCount ()+" )");

if (blnShowVisual) \{

generateVisualGraph (false);

\}

graphFrame.pack ();

break;

case "BURNING":

dominatingSet $=$ DominatingSet. burning $(\mathrm{g})$;

System.out.println (( dominatingSet.size ()$+1)+"$ rounds" );

System . out.println(dominatingSet.toString ());

if (blnShowVisual) \{

createGraphWithDS ( ) ;

\}

break;

\}

\}

public void saveGraphImage() \{

DateFormat dateFormat $=$ new SimpleDateFormat ("yyyy-MM-dd-HHmmss");

Date date $=$ new Date () ; 
String imageDate $=$ dateFormat format (date);

if $($ graphType $=$ GNR_GRAPH $)\{$ imageDate $+="-$ gnr";

\} else if (graphType $=$ GNP_GRAPH $)\{$ imageDate $+="-$ gnp";

\}

File outputfile = new File(imageDate+"-graph.png");

$\operatorname{try}\{$

ImageIO . write (image, "png", outputfile);

\} catch (IOException e) \{

// Exception handling

\}

\}

public static void exportSMATData() \{

BufferedWriter writer = null;

try \{

//create a temporary file

DateFormat dateFormat $=$ new SimpleDateFormat ("yyyy-MM-dd-HHmmss");

Date date $=$ new Date () ;

String fileDate = dateFormat.format (date);

if (graphType $=$ GNR_GRAPH) \{

fileDate $+="-$ gnr";

\} else if (graphType $=$ GNP_GRAPH) \{

fileDate $+="-$ gnp";

\}

File file = new File(fileDate+"-data.txt");

writer $=$ new BufferedWriter(new FileWriter(file)); 

$\arg 1)$;

Node [] $\operatorname{vtxs}=$ new Node $[$ g.getVertexCount () ];

g. getVertices (). toArray (vtxs);

Arrays. sort (vtxs, comp);

for (Node vtx : vtxs) \{

for (Node adj : vtx.getAdjacent ()) \{

writer.write $(\operatorname{adj} \cdot \operatorname{getID}()+"=+\mathrm{vtx} \cdot \operatorname{get} \mathrm{ID}())$;

writer newLine ( ) ;

$\operatorname{adj}$. deleteEdge (vtx);

\}

\}

\} $\operatorname{catch}($ IOException e) \{

//e.printStackTrace();

\} finally \{

try \{

writer. close ();

\} $\operatorname{catch}($ IOException e) \{

//e.printStackTrace ();

\}

\}

\}

public static void exportGraphData() \{

BufferedWriter writer = null;

try \{

//create a temporary file

DateFormat dateFormat $=$ new SimpleDateFormat ("yyyy-MM-dd-HHmmss");

Date date $=$ new Date () ;

String fileDate $=$ dateFormat.format (date); 


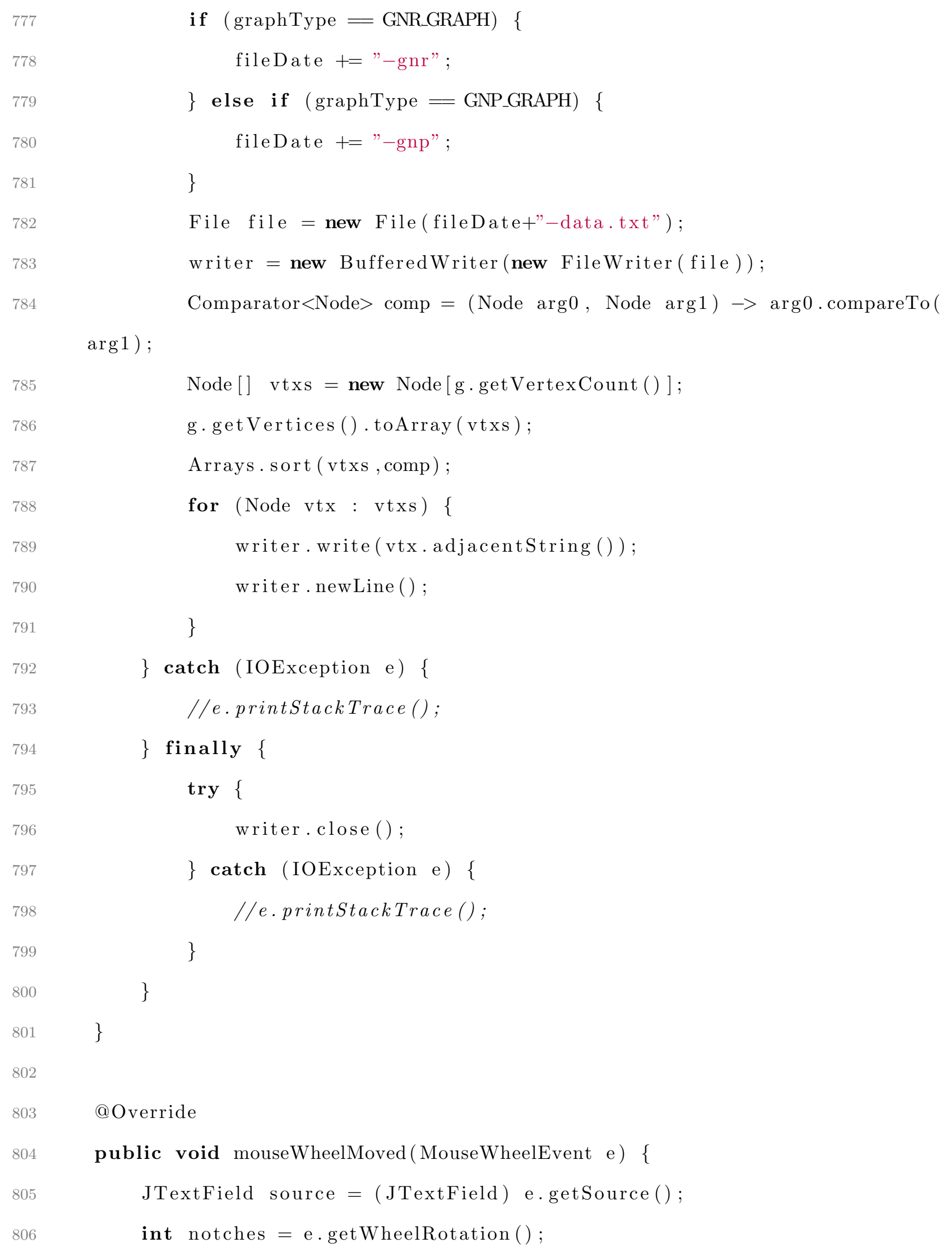

$$
\text { if (graphType }=\text { GNR_GRAPH) }\{
$$

$$
\text { fileDate }+="-\text { gnr"; }
$$$$
\} \text { else if (graphType }=\text { GNP_GRAPH })\{
$$

$$
\text { fileDate }+="-\text { gnp"; }
$$

\}

File file = new File(fileDate+"-data.txt");

writer $=$ new BufferedWriter (new FileWriter(file)) ;

Comparator $<$ Node $>\operatorname{comp}=($ Node $\arg 0$, Node $\arg 1) \rightarrow \arg 0 . \operatorname{compareTo}($ $\arg 1)$

$$
\text { Node [] vtxs }=\text { new Node }[g \cdot g e t V e r t e x C o u n t()] ;
$$$$
\text { g. getVertices (). toArray (vtxs); }
$$$$
\text { Arrays. sort (vtxs, comp); }
$$$$
\text { for (Node vtx: vtxs) \{ }
$$$$
\text { writer. write (vtx . adjacentString ()); }
$$ 
DecimalFormat $\mathrm{df}=$ new DecimalFormat ("\#.\#\#\#");

if (source.equals (txtNodes)) \{

if $($ notches $<0)\{$ txtNodes.setText (Integer.parseInt (txtNodes.getText ()$)+1+"$ "

)

\} else \{

txtNodes.setText(Integer $\cdot$ parseInt (txtNodes.getText ()$)-1+"$ "

)

$$
\text { \} }
$$

\} else if (source.equals $($ txtP $))\{$

if $($ notches $<0)\{$ txtP.setText (df.format (Double.parseDouble (txtP.getText ()$)+$

$0.05))$;

\section{\} else \{}

txtP.setText (df.format (Double.parseDouble (txtP.getText ()) -

$0.05))$;

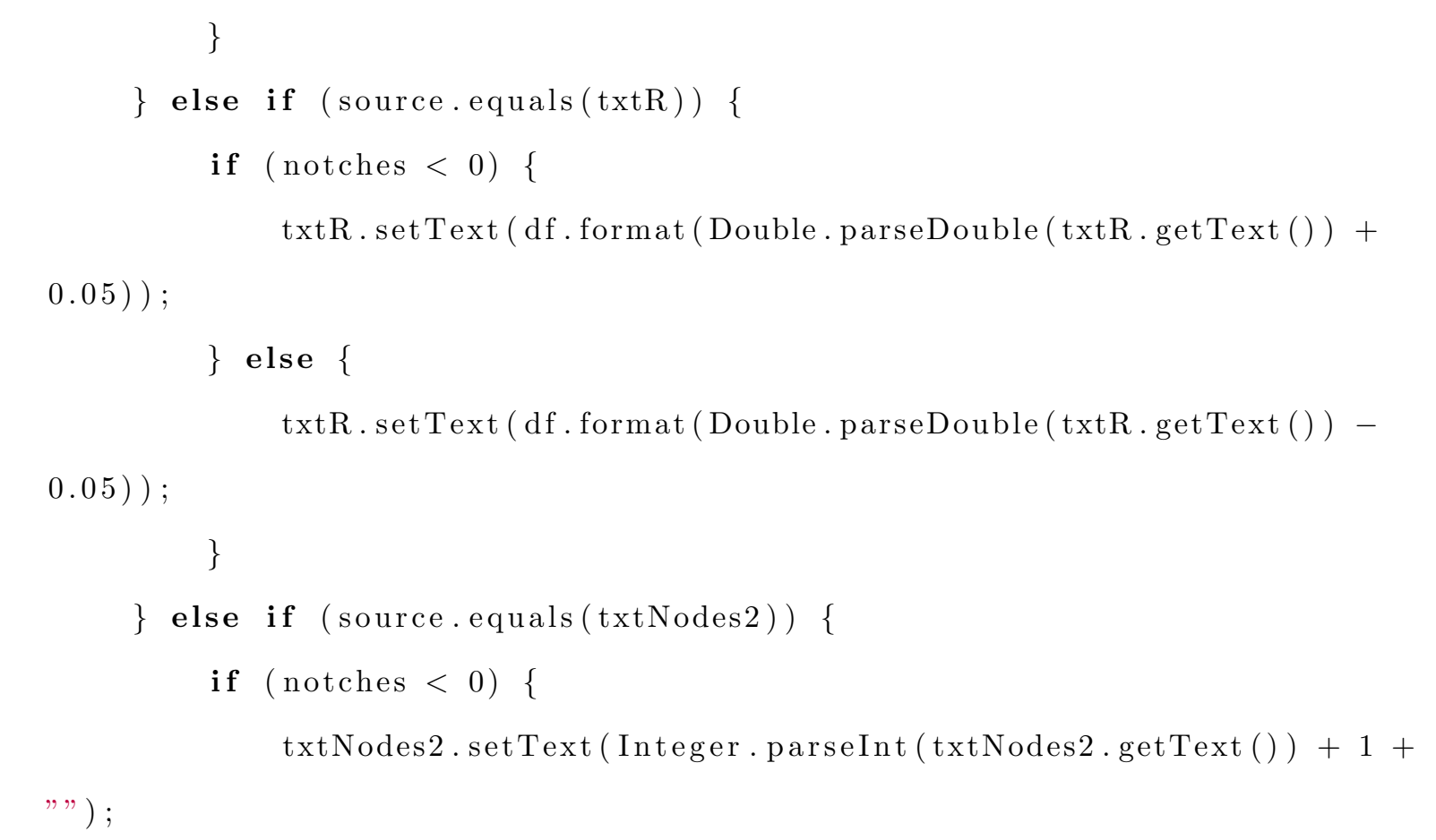


$"$ ") ;

831

832

833

834

835

836

837

838

839

840

841

842

843

844

845

846

847

848

849

850

851

852

854
\}

\} else if (source.equals $(\operatorname{txtN}))\{$

if $($ notches $<0)\{$

$\operatorname{txtN} \cdot \operatorname{setText}($ Integer $\cdot$ parseInt $(\operatorname{txtN} \cdot \operatorname{getText}())+1+\cdots ")$;

\} else \{

$\operatorname{txtN} \cdot \operatorname{set} T \operatorname{ext}($ Integer $\cdot$ parseInt $(\operatorname{txtN} \cdot \operatorname{getText}())-1+" ”)$;

\}

\} else if (source.equals (txtM) $)\{$

if $($ notches $<0)\{$

txtM.setText (Integer $\cdot$ parseInt (txtM $\cdot \operatorname{getText}())+1+" ”)$;

\} else \{

txtM.setText (Integer p parseInt (txtM.getText ()$)-1+" ”)$;

\}

\} else if (source.equals (txtAlpha)) \{

if $($ notches $<0)\{$

txtAlpha.setText (df.format (Double.parseDouble (txtAlpha.getText

()) +0.05$))$;

\} else \{

txtAlpha.setText (df.format (Double.parseDouble (txtAlpha.getText

( ) -0.05$))$;

\}

\} else if (source.equals (txtBeta)) \{

if $($ notches $<0)\{$

txtBeta.setText (df.format (Double.parseDouble(txtBeta.getText ()

)$+0.05))$;

\} else \{

txtBeta.setText (df.format (Double.parseDouble(txtBeta.getText ()

)$-0.05))$;

\} 


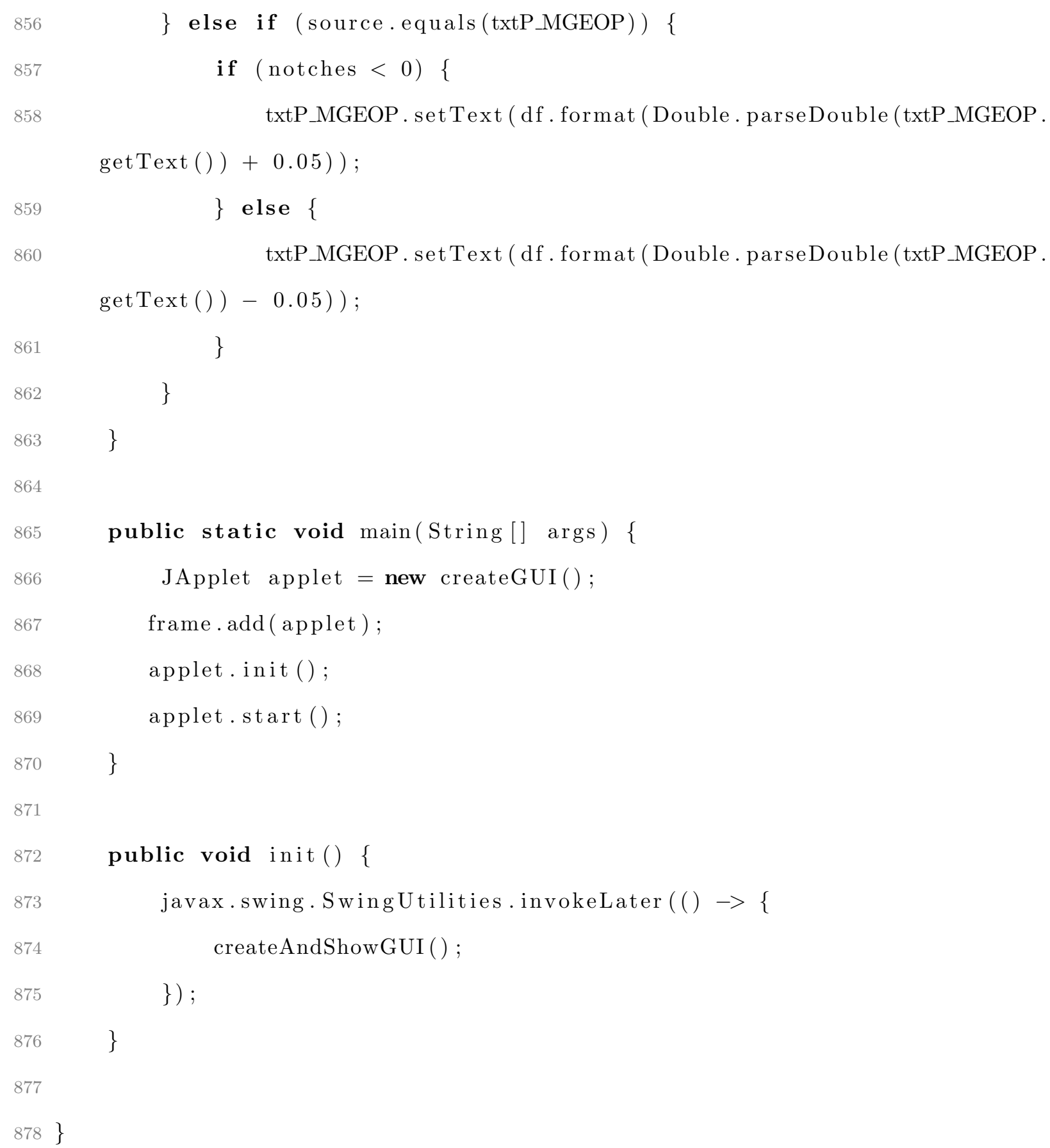

\} else if (source.equals (txtP_MGEOP $))\{$

if $($ notches $<0)\{$ txtP_MGEOP. set Text ( df . format (Double . parseDouble (txtP_MGEOP. $\operatorname{getText}())+0.05))$

\} else \{

txtP_MGEOP. setText ( df . format (Double . parseDouble (txtP_MGEOP. $\operatorname{get} \operatorname{Text}())-0.05))$;

public static void main(String [] args $)\{$

JApplet applet $=$ new createGUI ();

frame.add (applet);

applet.init ();

applet.start ();

\}

public void init () \{

javax.swing. Swing Utilities.invokeLater $(() \rightarrow\{$ createAndShowGUI ( ) ;

\}) ;

\}

\section{GraphProperties.java}

\footnotetext{
$1 / *$

2*@author Marc Lozier

$3 * /$

4
} 


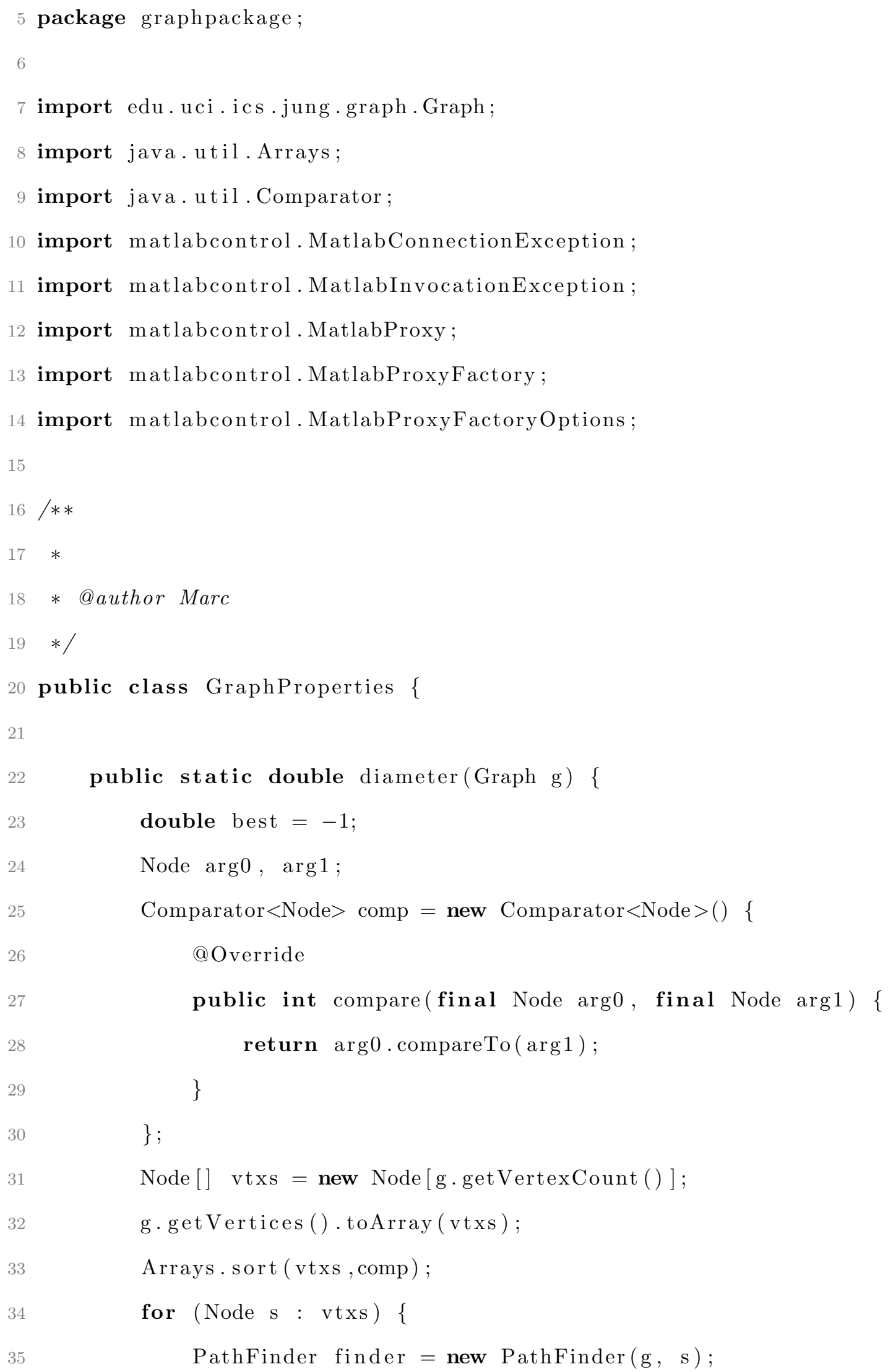


for (Node $\mathrm{v}:$ vtxs) \{

if (finder.isReachable(v) \&\& finder.distanceTo(v) > best) \{ best $=$ finder.distanceTo(v);

\}

if (v.getAdjacent () . isEmpty ()) \{ best $=$ Double.POSITIVE_INFINITY;

\}

\}

\}

return best;

\}

public static void degreeDistribution(Graph g) \{

int size $=$ g.getVertexCount ();

Node $\arg 0, \arg 1$;

Comparator $<$ Node $>$ comp $=$ new Comparator $<$ Node $>()\{$

@ Override

public int compare(final Node arg0, final Node $\arg 1)\{$ return $\arg 0 . c o m p a r e T o(\arg 1)$;

\}

\}

Node [] $\operatorname{vtxs}=$ new Node[size];

g.getVertices ().toArray (vtxs);

Arrays. sort (vtxs, comp);

String command $=$ "hist ([";

for (Node vtx : vtxs) \{ command $+=$ vtx $\cdot$ getDegree ()$+", "$;

\}

command $=$ command. substring $(0$, command. length ()$-1)$;

command $+="], "+$ size+")"; 


\section{GraphManipulation.java}

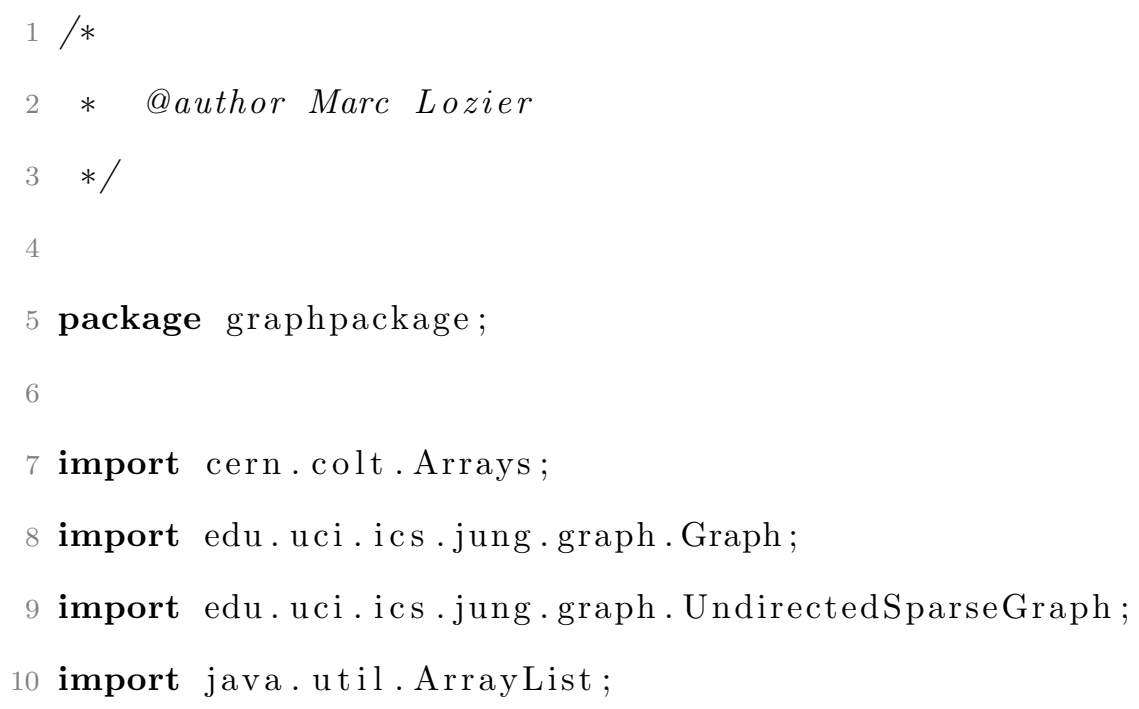


11 import java.util. List;

2 import java.util. Random;

13

$14 / * *$

$15 *$

16 * @author Marc

$17 * /$

18 public class GraphManipulation \{

public static UndirectedSparseGraph $<$ Node, String $>$ oneCore(

UndirectedSparseGraph $<$ Node, String $>$ oldGraph ) \{

UndirectedSparseGraph $<$ Node, String $>$ newGraph $=$ oldGraph;

Node [] nodes $=$ DominatingSet.graphToArray (newGraph);

for (Node $\mathrm{v}:$ nodes $)\{$

if (newGraph.degree $(\mathrm{v})=0)\{$ newGraph . removeVertex (v);

\}

\}

return newGraph;

\}

public static UndirectedSparseGraph $<$ Node, String $>$ twoCore(

UndirectedSparseGraph oldGraph) \{

UndirectedSparseGraph $<$ Node, String $>$ newGraph $=$ oldGraph;

Node [] nodes;

List $<$ Node $>$ toRemove $=$ new ArrayList $<$ Node $>()$;

boolean repeat $=$ true;

while (repeat) \{

toRemove.clear ();

repeat $=$ false

nodes $=$ DominatingSet.graphToArray (newGraph);

for (Node $\mathrm{v}$ : nodes ) \{ 
if (Integer parseInt (v.getDegree ()) $<2)\{$

for (Node $\mathrm{x}$ : v.getAdjacent ()) \{ x. deleteEdge (v); toRemove. add (x); newGraph . removeEdge (newGraph . findEdge (x, v ) ); repeat $=$ true;

\}

for (Node $\mathrm{w}$ : toRemove) \{ v. deleteEdge (w);

\} newGraph . removeVertex (v);

\} \} \} return newGraph;

\}

public static UndirectedSparseGraph $<$ Node, String $>$ threeCore ( UndirectedSparseGraph oldGraph) \{

UndirectedSparseGraph $<$ Node, String $>$ newGraph $=$ oldGraph;

Node [] nodes;

List $<$ Node $>$ toRemove $=$ new ArrayList $<$ Node $>() ;$

boolean repeat $=$ true;

while (repeat) \{

toRemove.clear ();

repeat $=$ false

nodes $=$ DominatingSet.graphToArray (newGraph);

for (Node $\mathrm{V}:$ nodes $)\{$

if (newGraph. degree $(\mathrm{v})<3$ ) \{

for (Node x : v.getAdjacent ()) \{

$\mathrm{x} . \operatorname{deleteEdge}(\mathrm{v})$; 
toRemove. add (x); newGraph . removeEdge (newGraph . findEdge (x, v)) ; repeat $=$ true; \} for (Node $\mathrm{w}$ : toRemove) \{ v.deleteEdge (w);

\} newGraph . removeVertex (v);

\} \} \} return newGraph;

\}

public static UndirectedSparseGraph $<$ Node, String $>$ kCore ( UndirectedSparseGraph oldGraph, int k) \{ UndirectedSparseGraph $<$ Node, String $>$ newGraph $=$ oldGraph; Node [] nodes ; List $<$ Node $>$ toRemove $=$ new ArrayList $<$ Node $>()$; boolean repeat $=$ true; while (repeat) \{ repeat $=$ false nodes $=$ DominatingSet.graphToArray (newGraph); for (Node $\mathrm{v}:$ nodes $)\{$ if (newGraph.degree $(\mathrm{v})<\mathrm{k})\{$ for (Node $\mathrm{x}$ : v.getAdjacent ()) \{ $\mathrm{x} \cdot \operatorname{deleteEdge}(\mathrm{v})$; toRemove . add $(\mathrm{x})$; newGraph . removeEdge (newGraph . findEdge (x, v ) ) ; repeat $=$ true;

\} 
for (Node w : toRemove) \{

v.deleteEdge (w);

\}

newGraph . removeVertex (v);

public static UndirectedSparseGraph $<$ Node, String $>$ percolateEdges ( UndirectedSparseGraph $<$ Node, String $>$ oldGraph, int edges $)\{$

UndirectedSparseGraph $<$ Node, String $>$ newGraph $=$ oldGraph;

Node [] nodes = DominatingSet.graphToArray (newGraph);

shuffleArray (nodes);

Random genRandom $=$ new Random $($ System $\cdot$ nanoTime ()$)$;

int toRemove $=$ newGraph.getEdgeCount ()$-$ edges ;

while (toRemove $>0)\{$

for (Node $\mathrm{v}:$ nodes $)$ \{

int currentDegree $=$ Integer $\cdot$ parseInt (v.getDegree ()) ;

if (currentDegree $=0$ ) continue;

int amountDelete $=$ genRandom. nextInt (currentDegree);

if (amountDelete $=0)$ continue;

List $<$ Node $>$ adjacent $=\mathrm{v} \cdot \operatorname{get} \operatorname{Adjacent}()$;

List $<$ Node $>$ deletedNodes $=$ new ArrayList $<$ Node $>()$;

while( amountDelete $>0)\{$

for (Node w : adjacent) \{

int toDelete $=$ genRandom. $\operatorname{nextInt}(2)$;

if $(($ toDelete $=1) \&($ amountDelete $>0))\{$

if (newGraph.findEdge $(\mathrm{w}, \mathrm{v})=$ null) continue; 


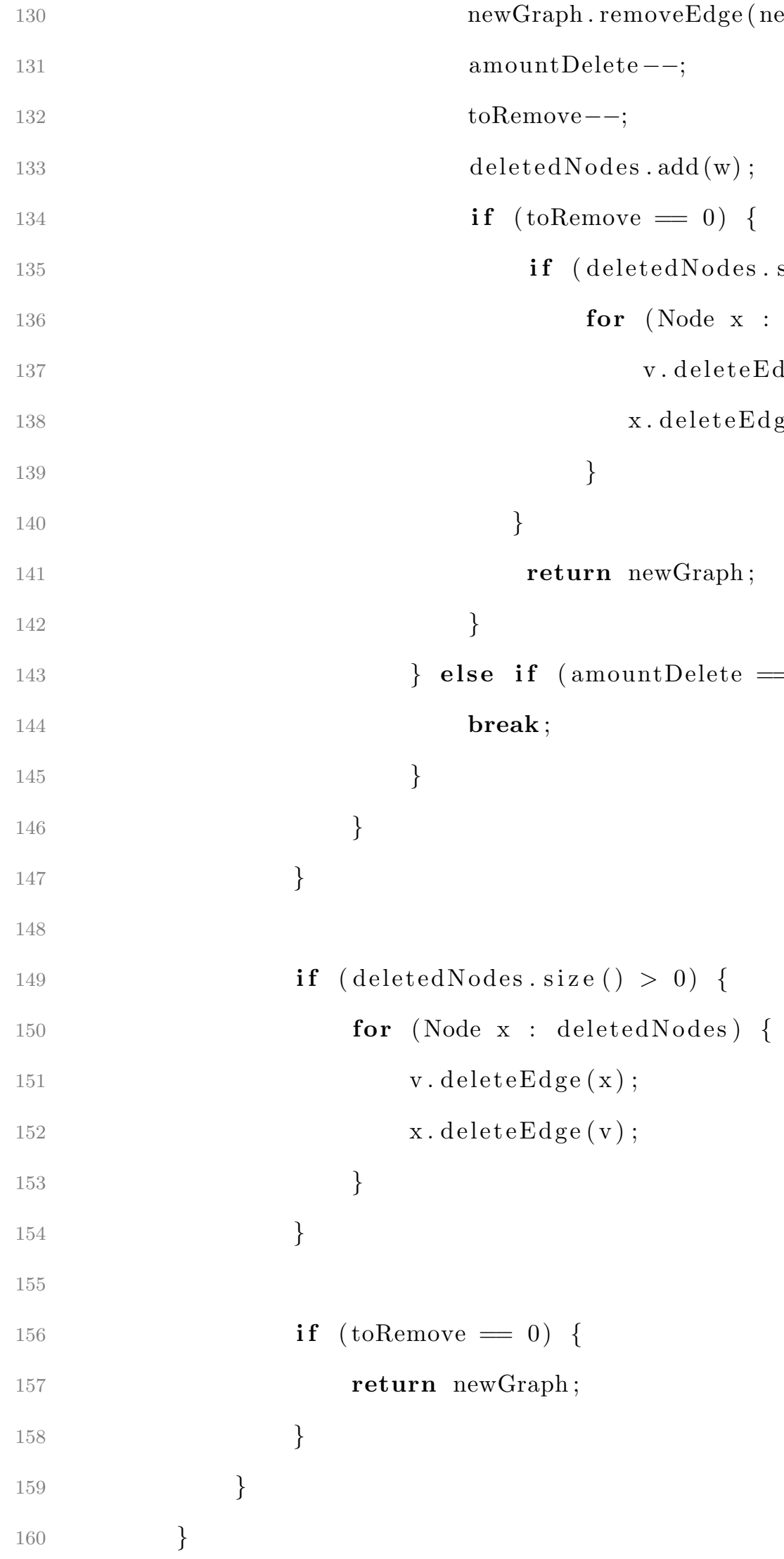




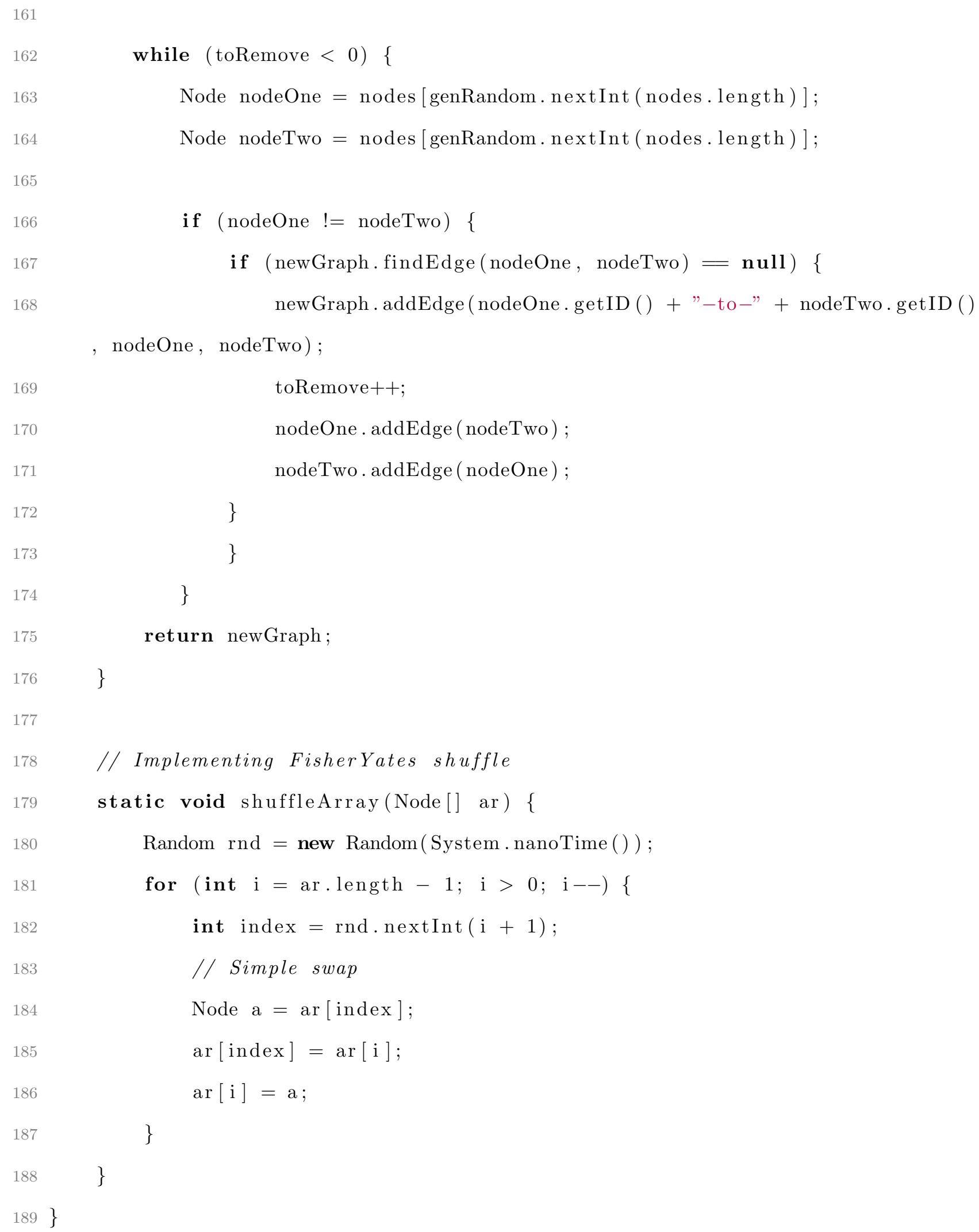

while (toRemove $<0)\{$

Node nodeOne $=$ nodes $[$ genRandom. nextInt $($ nodes. length $)]$;

Node $\operatorname{nodeTwo}=\operatorname{nodes}[$ genRandom. $\operatorname{nextInt}(\operatorname{nodes} . \operatorname{length})]$;

if (nodeOne $!=$ nodeTwo) \{

if (newGraph.findEdge (nodeOne, nodeTwo $)=\mathbf{n u l l}) \quad\{$ newGraph .addEdge (nodeOne.getID () + "-to-" + nodeTwo.getID ()

, nodeOne, nodeTwo); 


\section{FileReader.java}

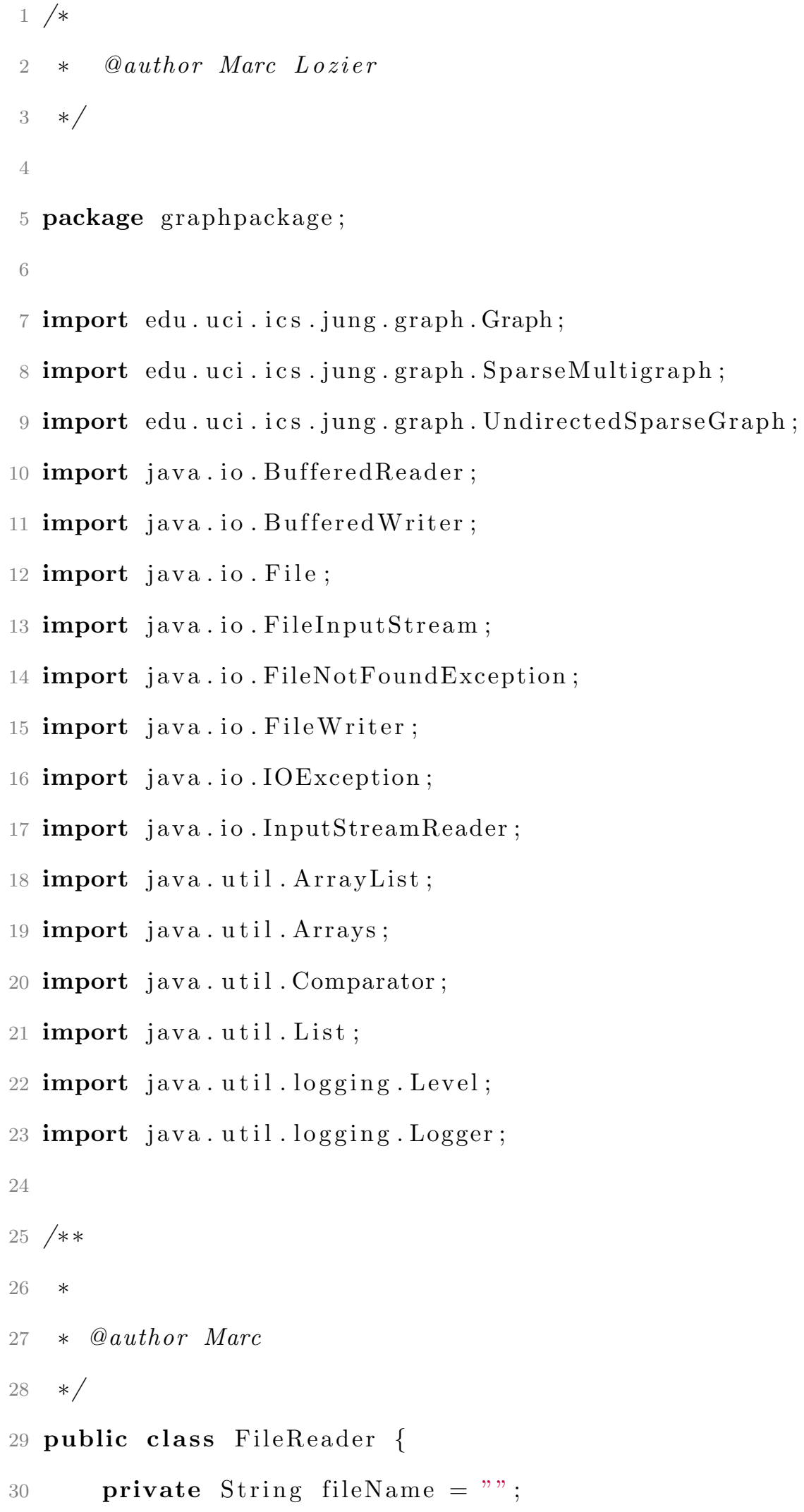




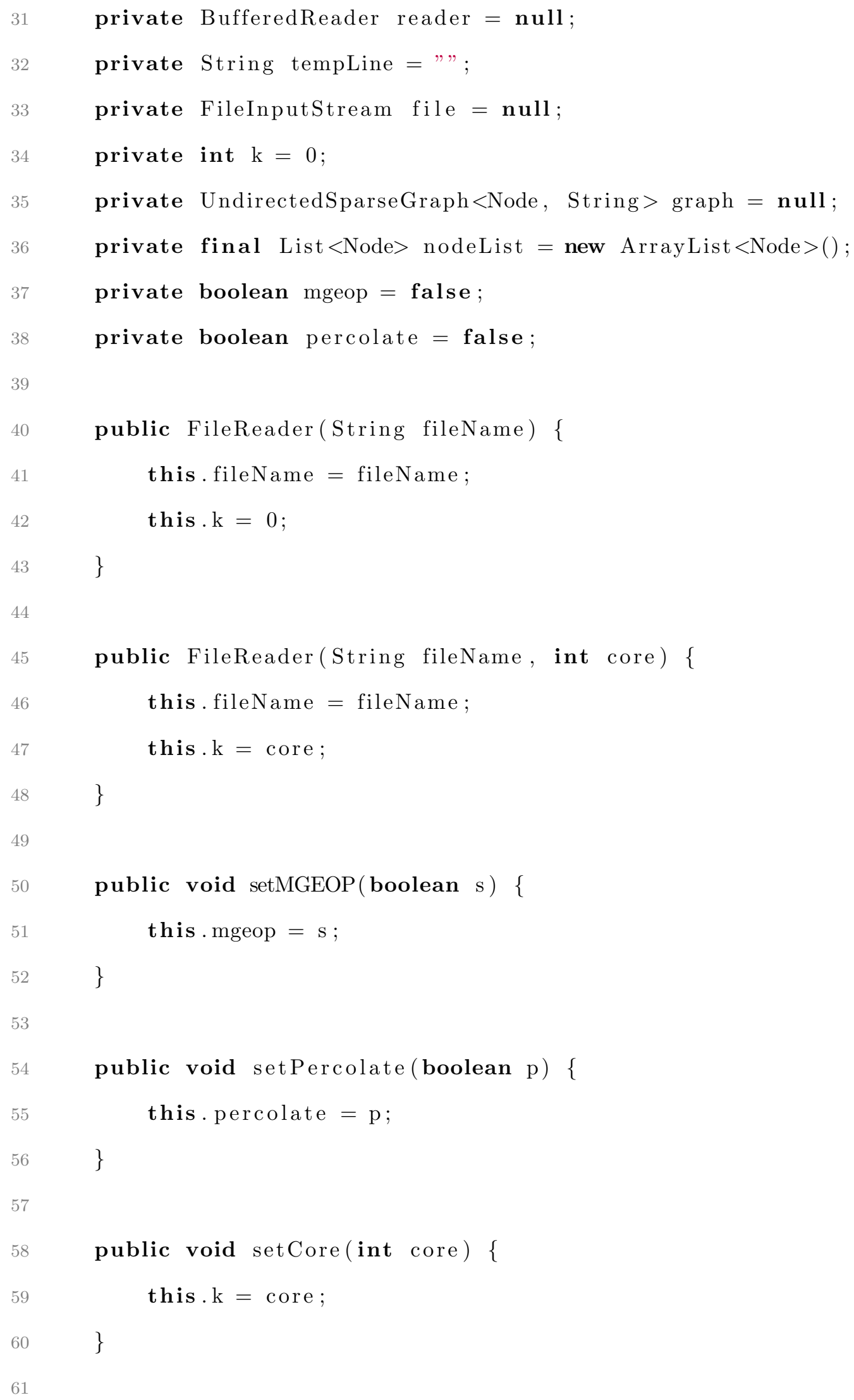



smat") ;

(2)

fileName = fileName.replace (".smat" , " _" + k+"_compressed.

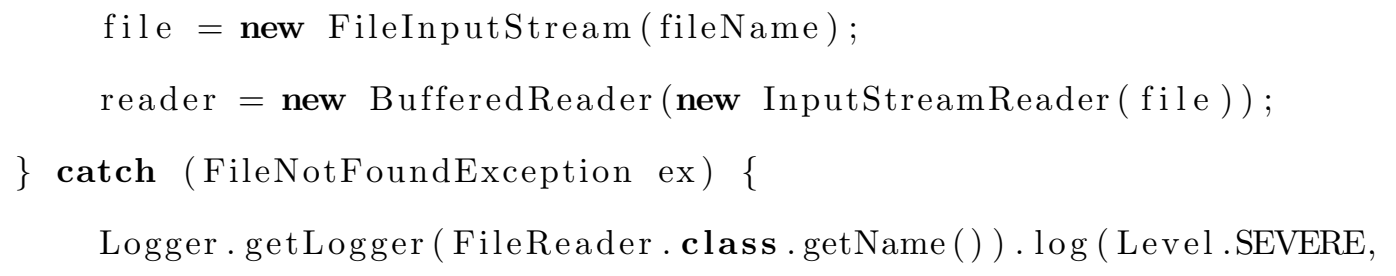




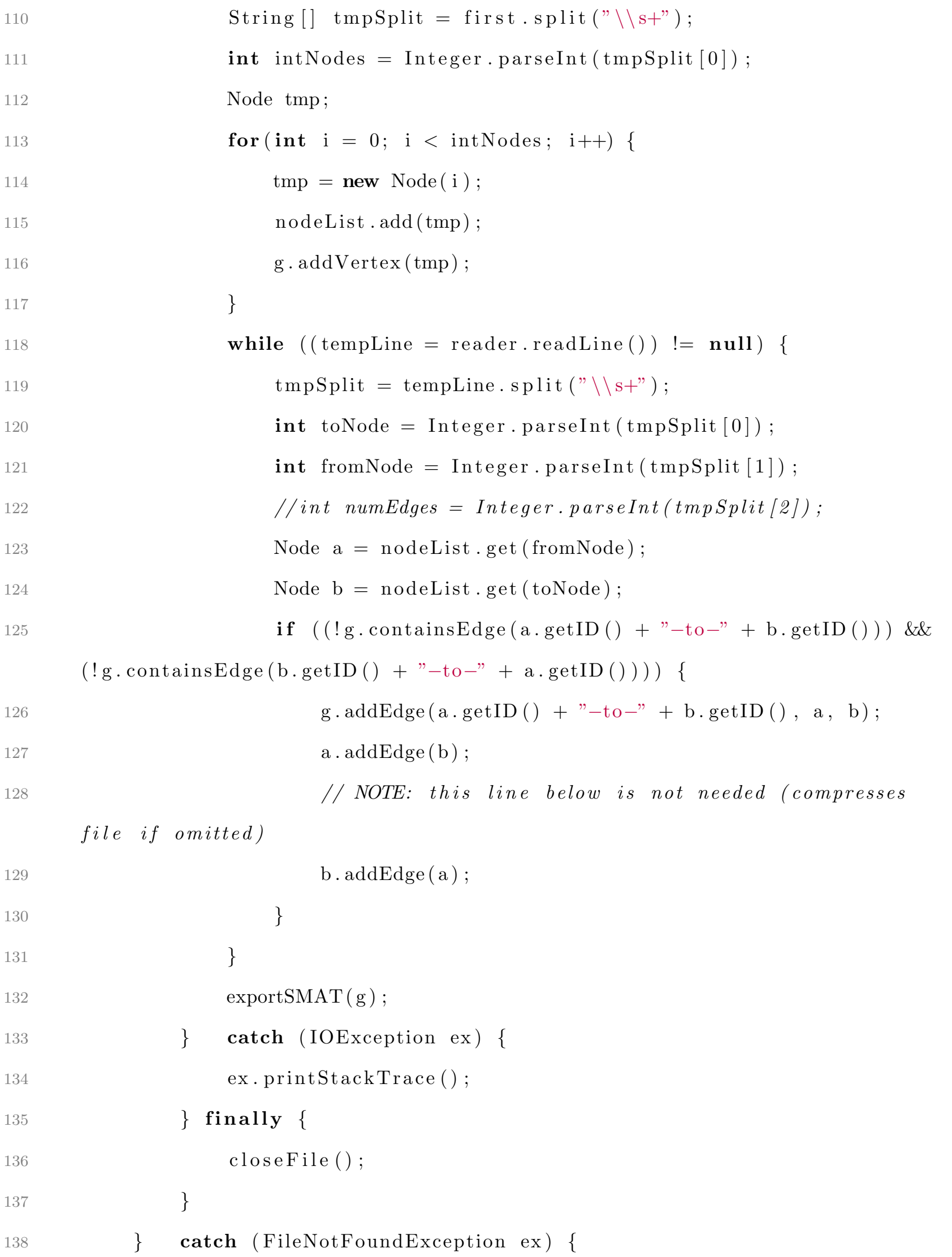

String [] tmpSplit $=$ first.split $(" \backslash \backslash \mathrm{s}+")$;

int intNodes $=$ Integer $\cdot$ parseInt $($ tmpSplit $[0])$;

Node tmp;

for $($ int $\mathrm{i}=0 ; \mathrm{i}<\operatorname{intNodes} ; \mathrm{i}++)\{$

tmp $=$ new Node $(\mathrm{i})$;

nodeList . add (tmp);

g. addVertex (tmp) ;

\}

while $(($ tempLine $=$ reader $\cdot$ readLine ()$) !=\mathbf{n u l l})\{$

tmpSplit $=$ tempLine. split $(" \backslash \backslash \mathrm{s}+")$;

int toNode $=$ Integer $\cdot$ parseInt (tmpSplit [0]);

int fromNode $=$ Integer $\cdot \operatorname{parseInt}(\operatorname{tmpSplit}[1])$;

$/ /$ int numEdges $=$ Integer.parseInt(tmpSplit[2]);

Node $\mathrm{a}=$ nodeList.get $($ fromNode $)$;

Node $\mathrm{b}=$ nodeList $\cdot \operatorname{get}($ toNode $)$;

if ( ( ! g.containsEdge (a.getID ()$+"-$ to $-"+b \cdot \operatorname{get}+\mathrm{DD}())) \& \&$

$(!$ g. containsEdge $(b \cdot \operatorname{getID}()+"-t o-"+a \cdot \operatorname{getID}())))\{$

$\mathrm{g} \cdot \operatorname{addEdge}(\mathrm{a} \cdot \operatorname{getID}()+"-\mathrm{to}-"+\mathrm{b} \cdot \operatorname{getID}(), \mathrm{a}, \mathrm{b})$;

a. addEdge (b);

// NOTE: this line below is not needed (compresses

file if omitted)

(1)


null, ex ) ;

\section{public void compressFile() \{}

UndirectedSparseGraph $<$ Node, String $>$ g $=$ new UndirectedSparseGraph $<$ Node , String $>()$;

readFile ();

try \{

nodeList. clear ();

String first $=$ reader readLine ()$;$

String [] tmpSplit $=$ first.split $(" \backslash \backslash s+")$;

int intNodes $=$ Integer $\cdot$ parseInt $($ tmpSplit $[0])$;

Node tmp;

for (int $\mathrm{i}=0 ; \mathrm{i}<\operatorname{intNodes} ; \mathrm{i}++)\{$

tmp $=$ new Node $($ i $) ;$

nodeList . add (tmp);

g. addVertex (tmp);

\}

while $(($ tempLine $=$ reader $\cdot$ readLine ()$) !=$ null $)\{$

tmpSplit = tempLine.split ("\\s+");

int toNode $=$ Integer $\cdot$ parseInt $(\operatorname{tmpSplit}[0])$;

int fromNode $=$ Integer.parseInt (tmpSplit [1]);

$/ /$ int numEdges $=$ Integer.parseInt (tmpSplit [2]);

Node $\mathrm{a}=$ nodelist.get (fromNode);

Node $\mathrm{b}=$ nodeList.get (toNode);

if $((! g$. containsEdge (a.getID ()$+"-t o-"+b \cdot g e t I D())) \& \&(! g$. containsEdge(b.getID () + "-to-" + a.getID ()) ) \{

$$
\text { g.addEdge (a.getID }()+"-\text { to-" + b.getID }(), \text { a , b ) ; }
$$


a . addEdge (b);

// NOTE: this line below is not needed (compresses file if

omitted)

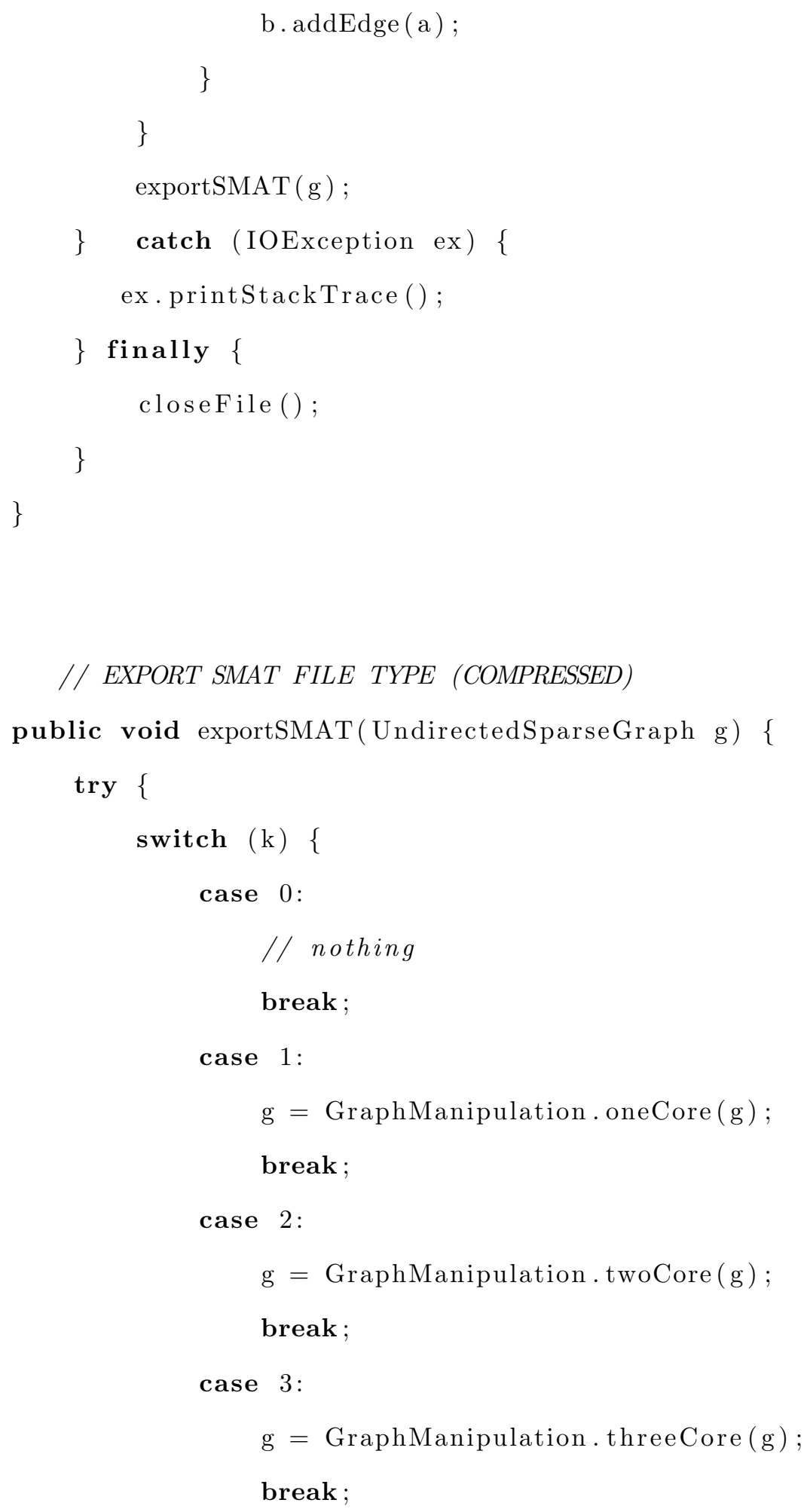




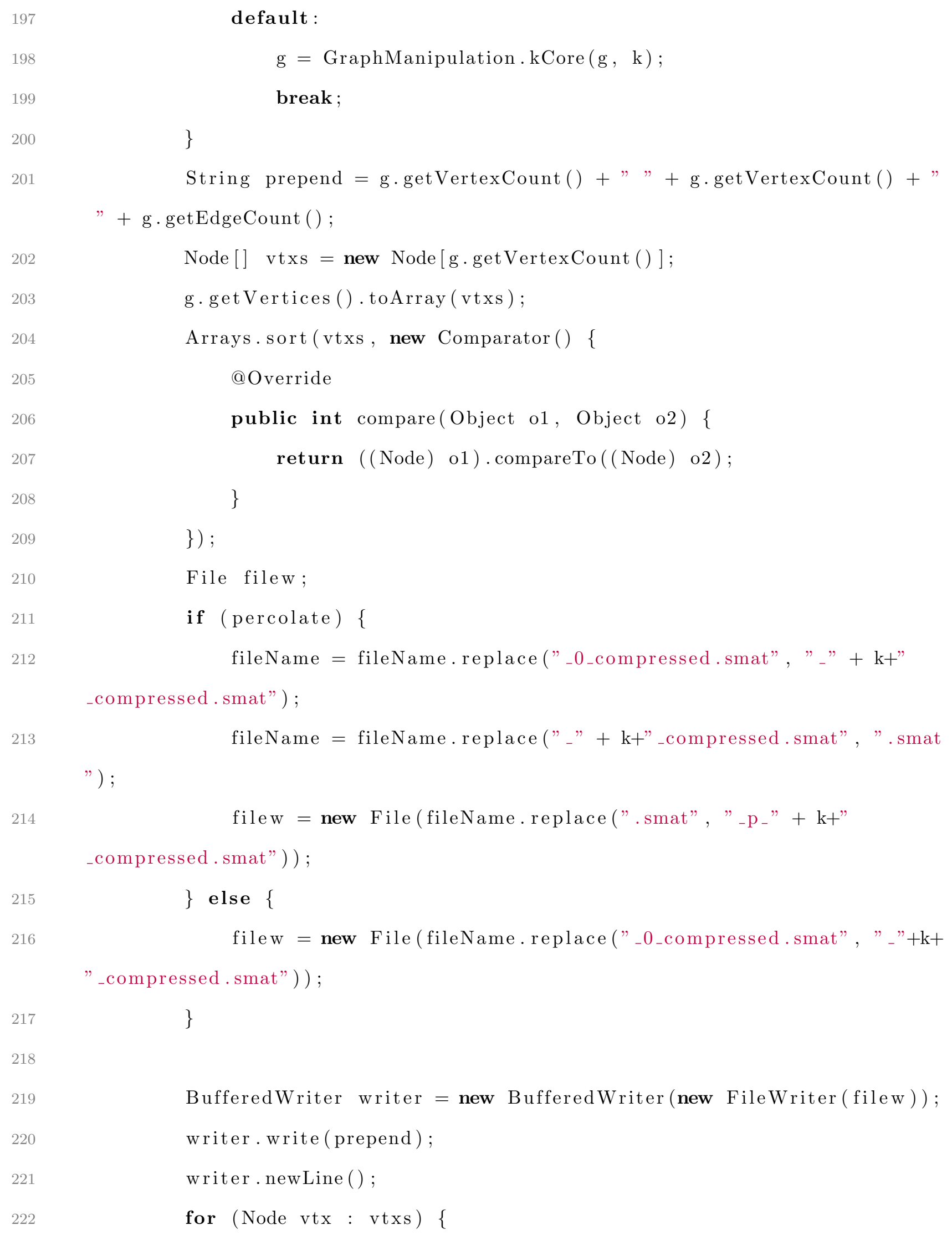

default : 


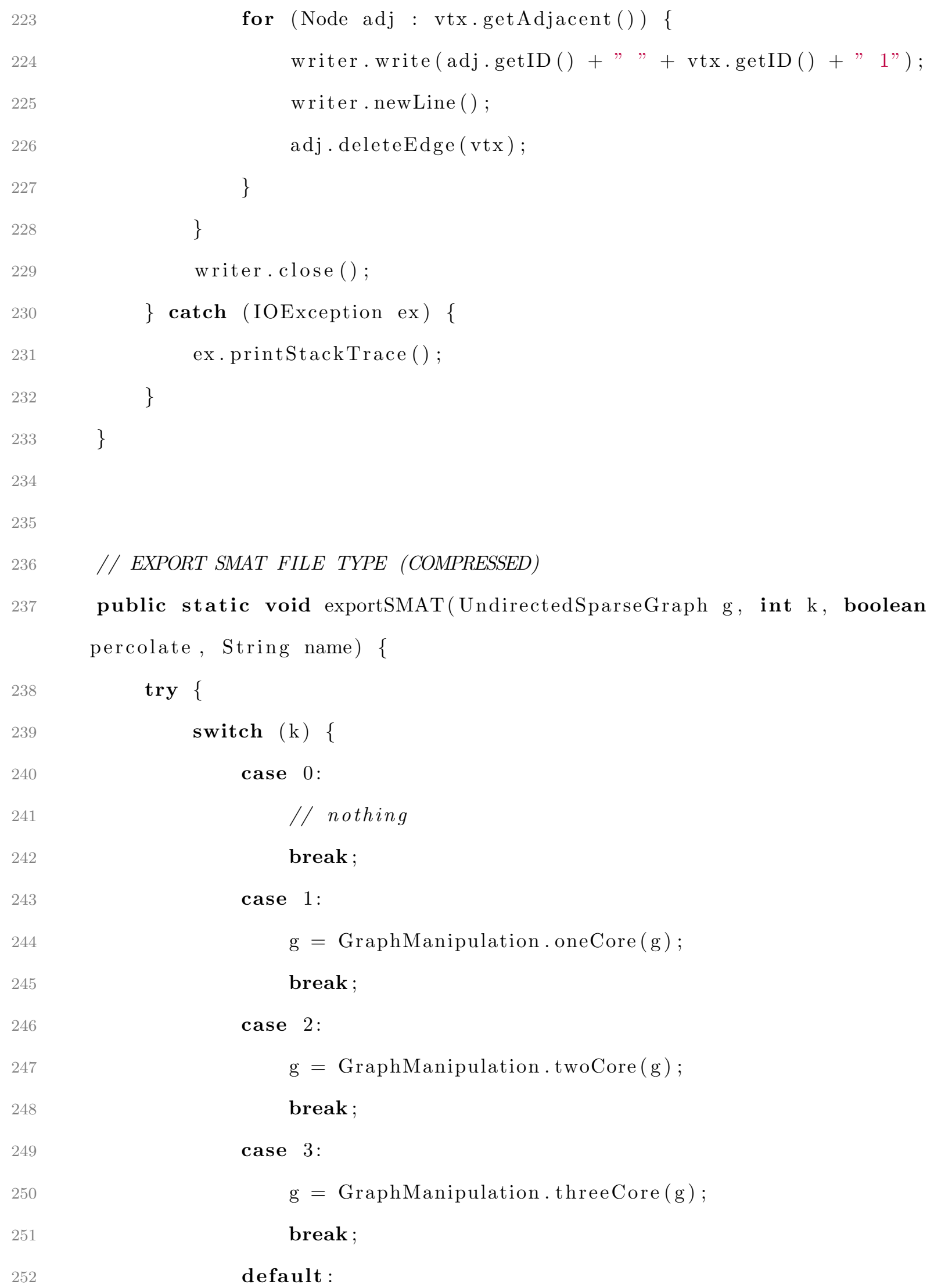

for (Node adj : vtx.getAdjacent ()$)\{$ writer.write $(\operatorname{adj} \cdot \operatorname{getID}()+"=+\operatorname{vtx} \cdot \operatorname{getID}()+" 1 ")$; writer. newLine ( ) ; adj. deleteEdge (vtx);

\}

\}

writer. close ();

\} $\operatorname{catch}($ IOException ex $)\{$ ex.printStackTrace ();

\}

\}

// EXPORT SMAT FILE TYPE (COMPRESSED)

public static void exportSMAT(UndirectedSparseGraph g, int $\mathrm{k}$, boolean percolate, String name) \{

$\operatorname{try}\{$

switch (k) \{

case 0 :

// nothing

break ;

case 1:

$\mathrm{g}=$ GraphManipulation . oneCore (g) ;

break ;

case 2:

$\mathrm{g}=$ GraphManipulation.twoCore (g);

break;

case 3 :

$\mathrm{g}=$ GraphManipulation . threeCore $(\mathrm{g})$;

break ;

default : 


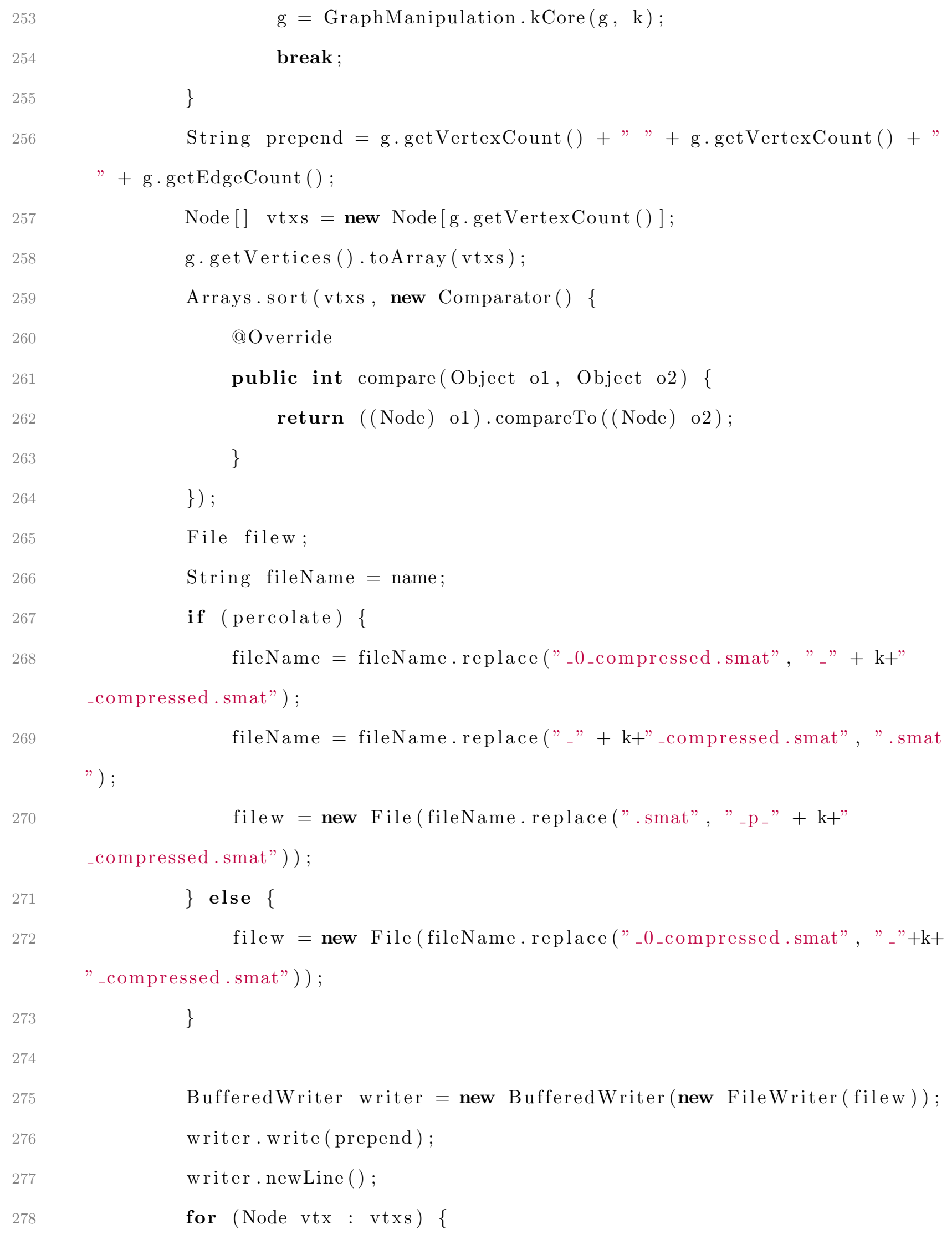

$\mathrm{g}=$ GraphManipulation $\cdot \mathrm{k} \operatorname{Core}(\mathrm{g}, \mathrm{k})$;

break ;

\}

String prepend $=$ g.getVertexCount ()$+"=+$ g.getVertexCount ()$+"$ $"+$ g.getEdgeCount () ;

Node [] $\operatorname{vtxs}=$ new Node $[\mathrm{g} \cdot \operatorname{getVertexCount}()]$;

g. getVertices (). toArray (vtxs);

Arrays.sort(vtxs, new Comparator () \{

@ Override

public int compare (Object o1, Object o2) \{

return (( Node) o1). compareTo ((Node) o2);

\}

\}) ;

File filew;

String fileName = name;

if (percolate) \{

fileName = fileName.replace ("_0_compressed.smat", " _" + k+" _compressed.smat");

fileName = fileName.replace (" _" + k+"_compressed.smat" , ".smat $")$ 


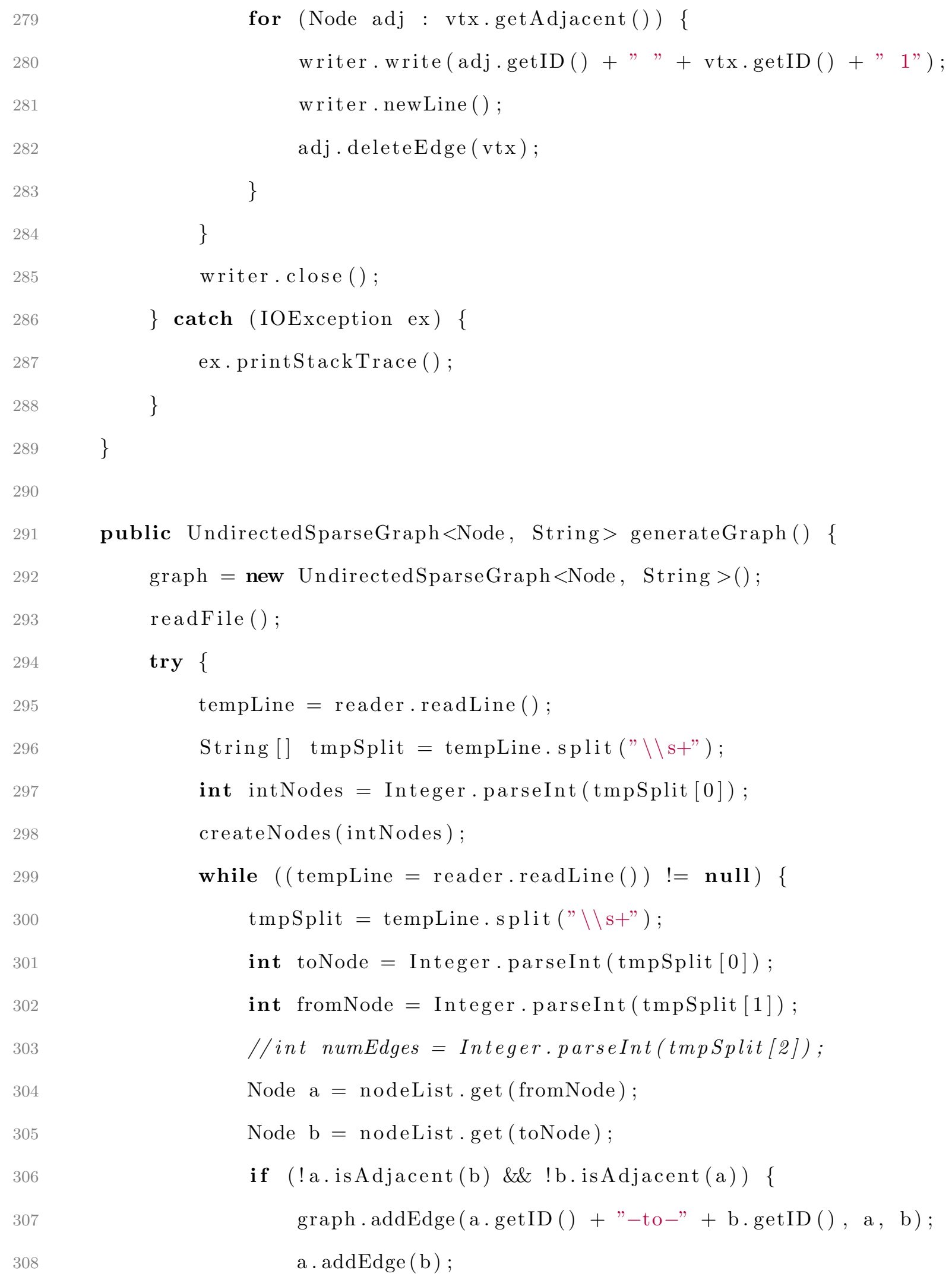

for (Node adj : vtx.getAdjacent ()$)\{$ writer.write $(\operatorname{adj} \cdot \operatorname{getID}()+"=+\operatorname{vtx} \cdot \operatorname{getID}()+" 1 ")$; writer. newLine ( ) ; adj . deleteEdge (vtx);

\} \} writer. close ();

\} $\operatorname{catch}($ IOException ex $)\{$ ex.printStackTrace ();

\} \} public UndirectedSparseGraph $<$ Node, String $>$ generateGraph ()\{ graph $=$ new UndirectedSparseGraph $<$ Node, String $>()$; readFile ();

try \{ tempLine $=$ reader $\cdot$ readLine ()$;$ String [] tmpSplit $=$ tempLine.split $(" \backslash \backslash \mathrm{s}+")$; int intNodes $=$ Integer $\cdot$ parseInt (tmpSplit [0]); createNodes (int Nodes); while $(($ tempLine $=$ reader. readLine ()$) !=$ null $)\{$ tmpSplit $=$ tempLine.split $(" \backslash \backslash \mathrm{s}+")$; int toNode $=$ Integer.parseInt (tmpSplit [0]); int fromNode $=$ Integer.parseInt (tmpSplit [1]); //int numEdges $=$ Integer.parseInt(tmpSplit[2]); Node $\mathrm{a}=$ nodeList.get (fromNode); Node $\mathrm{b}=$ nodeList $\cdot \operatorname{get}($ toNode $)$; if (!a.isAdjacent (b) \&\& ! b. isAdjacent (a)) \{ graph $\cdot \operatorname{addEdge}(a \cdot \operatorname{getID}()+"-t o-"+b \cdot \operatorname{getID}(), \mathrm{a}, \mathrm{b})$; a . addEdge (b) ; 
omitted)

\section{DominatingSet.java}

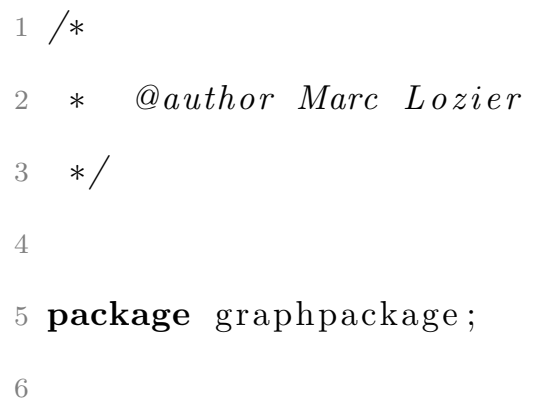




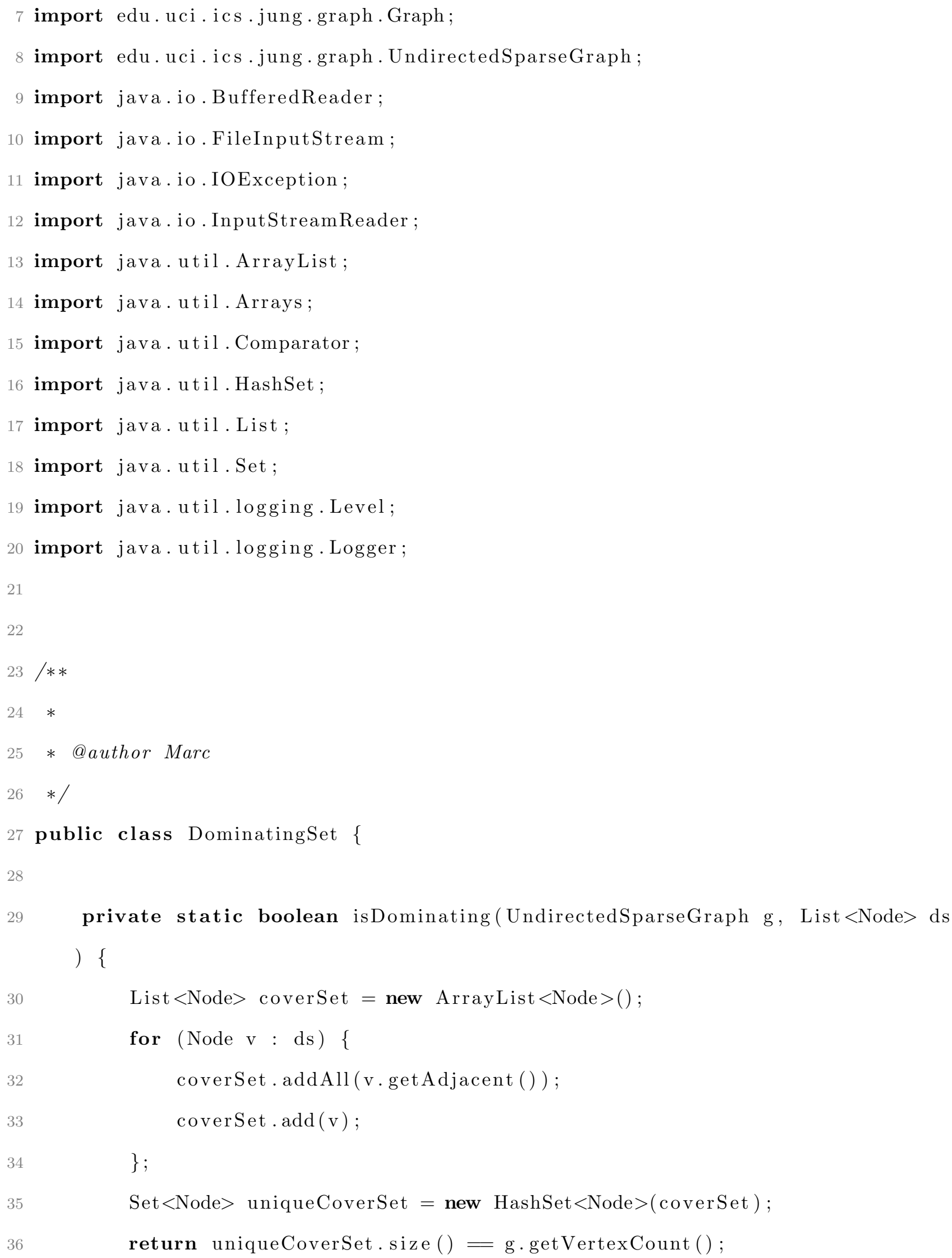




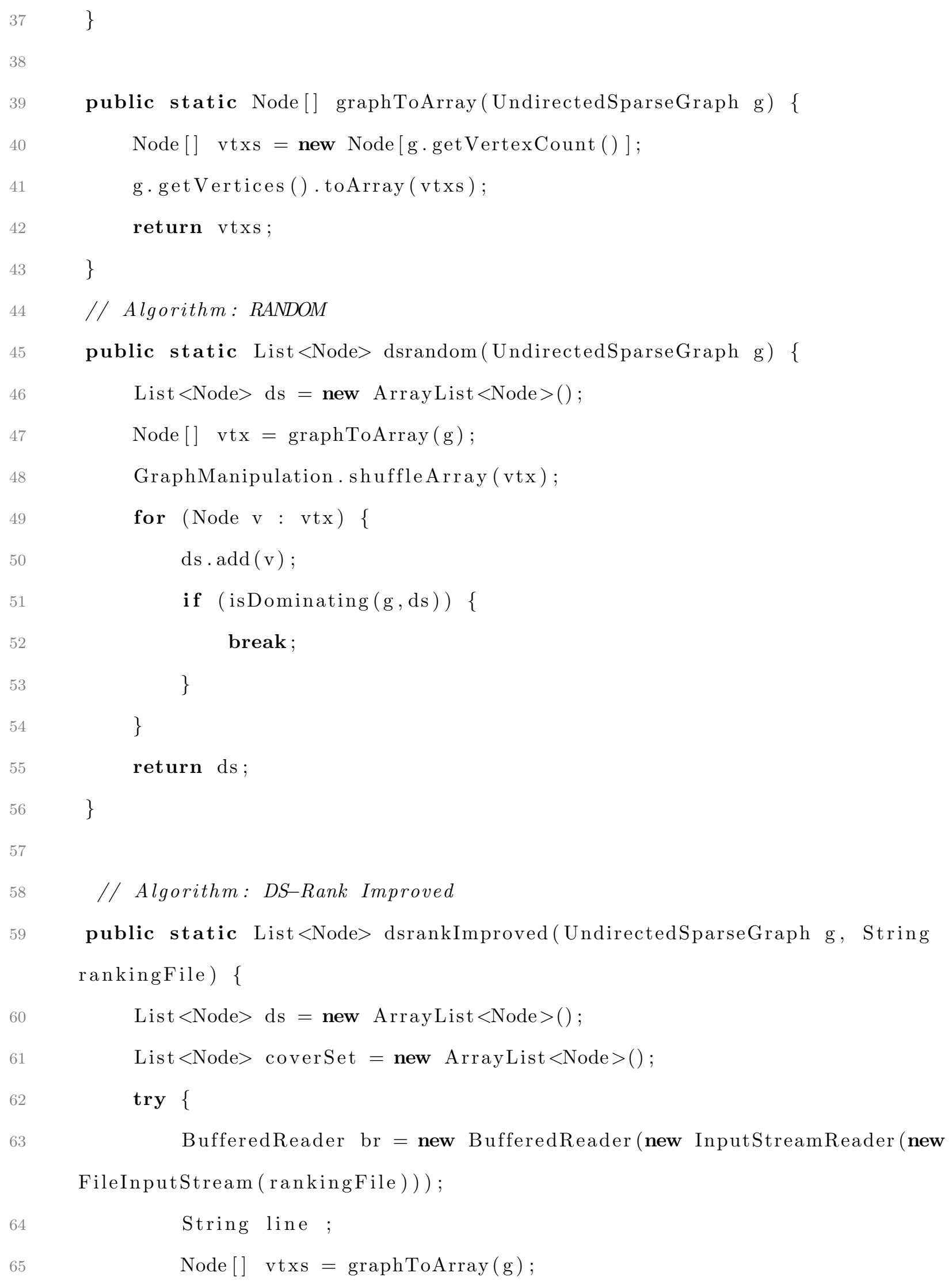

\section{\}}

public static Node[] graphToArray(UndirectedSparseGraph g) \{

Node [] $\operatorname{vtxs}=$ new Node[g.getVertexCount () ];

g.getVertices (). toArray (vtxs);

return vtxs;

\}

// Algorithm: RANDOM

public static List $<$ Node $>$ dsrandom(UndirectedSparseGraph g) \{

List $<$ Node $>$ ds $=$ new ArrayList $<$ Node $>()$

Node [] $\mathrm{vtx}=\operatorname{graphToArray}(\mathrm{g})$;

GraphManipulation.shuffleArray (vtx);

for (Node $\mathrm{v}: \mathrm{vtx})\{$

ds $\cdot \operatorname{add}(\mathrm{v})$;

if (isDominating $(\mathrm{g}, \mathrm{ds}))\{$

break ;

\}

\}

return ds;

\}

// Algorithm: DS-Rank Improved

public static List $<$ Node $>$ dsrankImproved(UndirectedSparseGraph g, String rankingFile ) \{

List $<$ Node $>$ ds $=$ new ArrayList $<$ Node $>() ;$

List $<$ Node $>$ coverSet $=$ new ArrayList $<$ Node $>()$;

$\operatorname{try}\{$

BufferedReader br = new BufferedReader (new InputStreamReader $($ new FileInputStream (rankingFile )) );

String line ;

Node [] $\operatorname{vtxs}=\operatorname{graphToArray}(\mathrm{g})$; 
while $(($ line $=$ br. readLine ()$) !=$ null $)\{$

for (Node $\mathrm{v}: \mathrm{vtxs})\{$ if (String.valueOf(v.getID ()) . equalsIgnoreCase(line)) \{

if (! coverSet. contains (v)) \{

$\mathrm{ds} \cdot \operatorname{add}(\mathrm{v})$;

coverSet . add (v);

coverSet . addAll (v.getAdjacent ());

break; // break out of for loop - only 1 node with

that ID

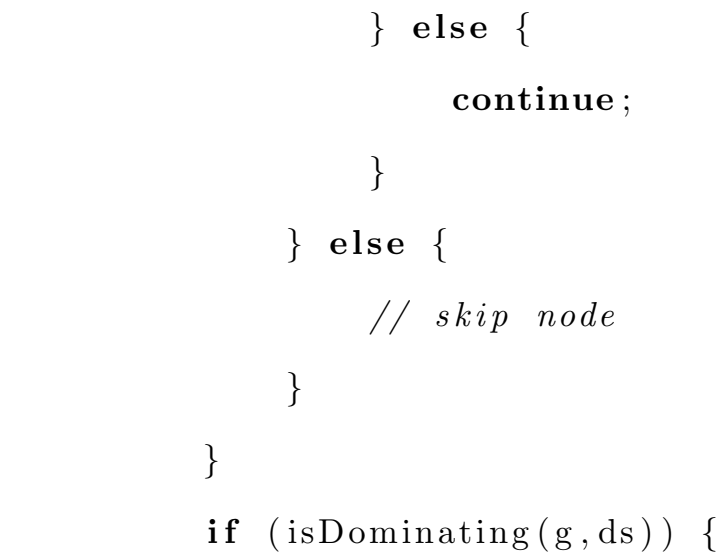

break; // leave file, found DS!

\}

\}

br. close ();

\} catch (IOException ex $)\{$

Logger . getLogger (DominatingSet . class .getName()) . log ( Level .SEVERE,

null, ex ) ;

return ds;

\}

// Algorithm: DS-Rank

public static List $<$ Node $>$ dsrank(UndirectedSparseGraph g, String rankingFile ) \{ 
List $<$ Node $>$ ds $=$ new ArrayList $<$ Node $>()$;

try \{

BufferedReader br = new BufferedReader(new InputStreamReader (new

FileInputStream (rankingFile)));

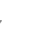

String line ;

Node [ ] vtxs $=$ graphToArray $(\mathrm{g})$;

while (( line $=$ br. readLine ()$) !=$ null $)\{$

for (Node $\mathrm{v}$ : vtxs) \{

if (String.valueOf (v.getID ( ) ) equalsIgnoreCase(line )) \{ ds . add (v);

break; // break out of for loop - only 1 node with

that ID

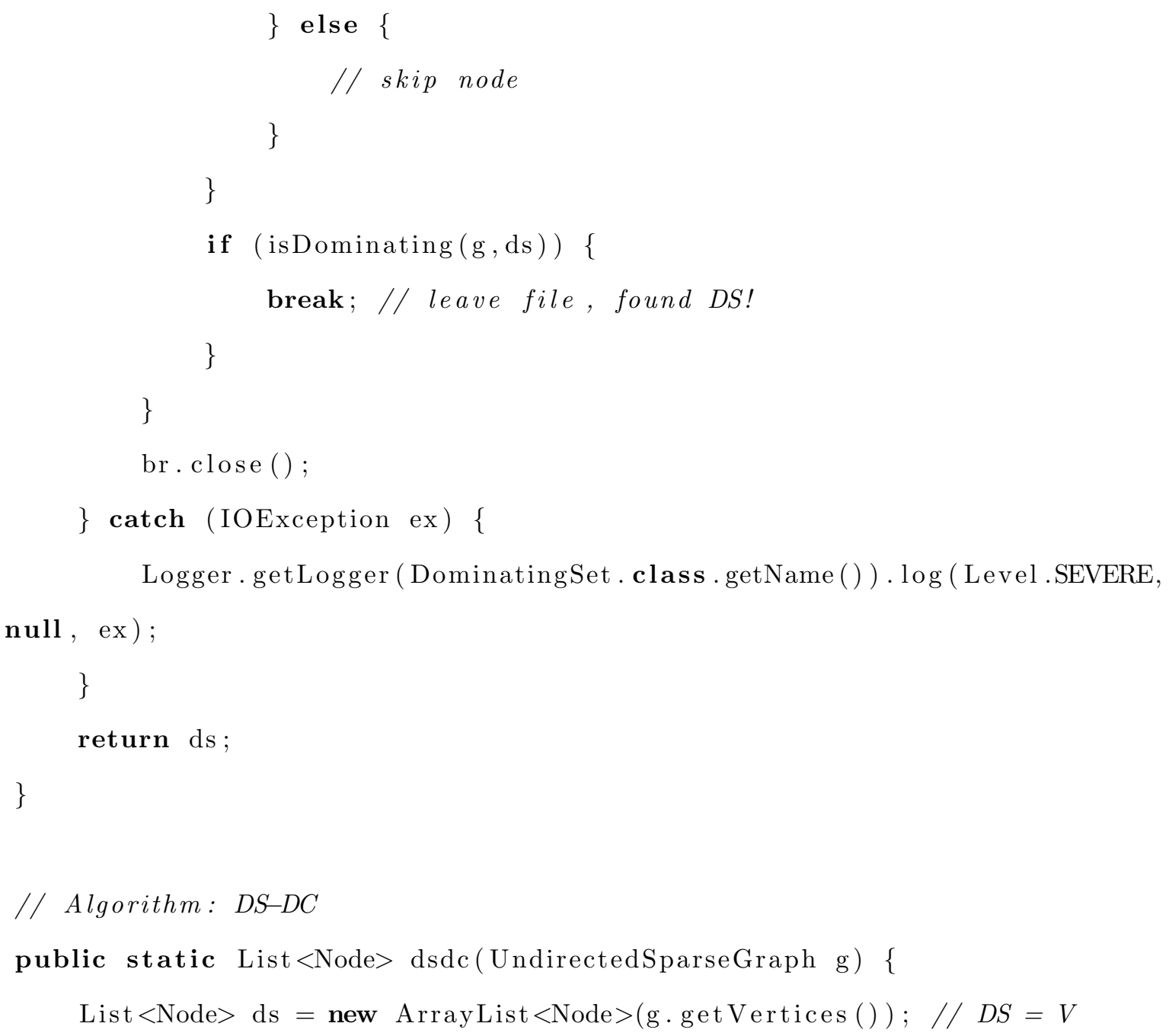


Node $\arg 0, \arg 1$;

Comparator $<$ Node $>$ comp $=$ new Comparator $<$ Node $>()\{$ @ Override

public int compare(final Node $\arg 0$, final Node $\arg 1)\{$ return $\arg 0$. compareDegreeToAsc $(\arg 1)$;

\}

\}

Node [] $\operatorname{vtxs}=$ graphToArray $(\mathrm{g}) ;$

Arrays.sort (vtxs, comp);

for (Node $\mathrm{v}: \mathrm{vtxs})\{$

$/ / v$ is the minimum node

ds.remove $(\mathrm{v})$;

if (! isDominating $(\mathrm{g}, \mathrm{ds}))\{$ ds . add (v);

\}

\}

return ds;

\}

\section{// Algorithm: DS-RAI}

public static List $<$ Node $>$ dsrai (UndirectedSparseGraph g, int phase) \{ List $<$ Node $>$ ds ;

List $<$ Node $>$ black $=$ new ArrayList $<$ Node $>()$;

List $<$ Node $>$ gray $=$ new ArrayList $<$ Node $>()$;

if $($ phase $=1)\{$

// phase 1

Node $\arg 0, \arg 1$;

Comparator $<$ Node $>$ comp $=$ new Comparator $<$ Node $>$ ( ) \{

@ Override

public int compare(final Node $\arg 0$, final Node $\arg 1)\{$ 


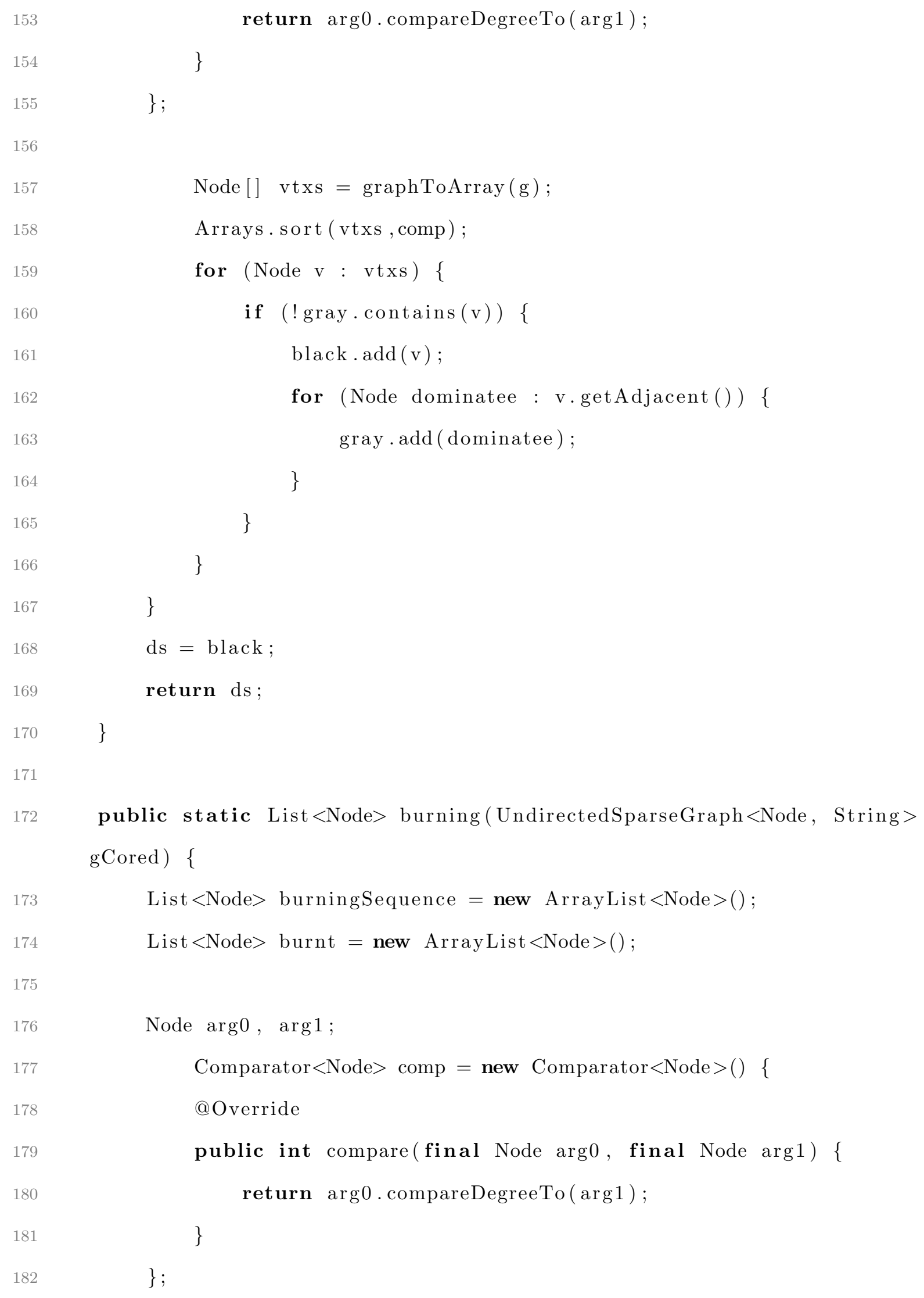

return arg0.compareDegreeTo $(\arg 1)$;

\}

\}

Node [] vtxs $=\operatorname{graphToArray}(\mathrm{g})$;

Arrays. sort (vtxs, comp);

for (Node $v: \operatorname{vtxs}) \quad\{$

if (! gray.contains (v)) \{

black $\cdot \operatorname{add}(\mathrm{v})$;

for (Node dominatee : v.getAdjacent ()) \{

gray $\cdot \operatorname{add}($ dominatee ) ;

\}

\}

\}

\}

$\mathrm{ds}=\mathrm{black}$

return ds;

\}

public static List $<$ Node $>$ burning (UndirectedSparseGraph $<$ Node, String $>$ gCored ) \{

List $<$ Node $>$ burningSequence $=$ new ArrayList $<$ Node $>()$;

List $<$ Node $>$ burnt $=$ new ArrayList $<$ Node $>()$;

Node $\arg 0, \arg 1$

Comparator $<$ Node $>$ comp $=$ new Comparator $<$ Node $>() \quad\{$

@Override

public int compare(final Node $\arg 0$, final Node $\arg 1)\{$

return arg0.compareDegreeTo $(\arg 1)$;

\}

\} 


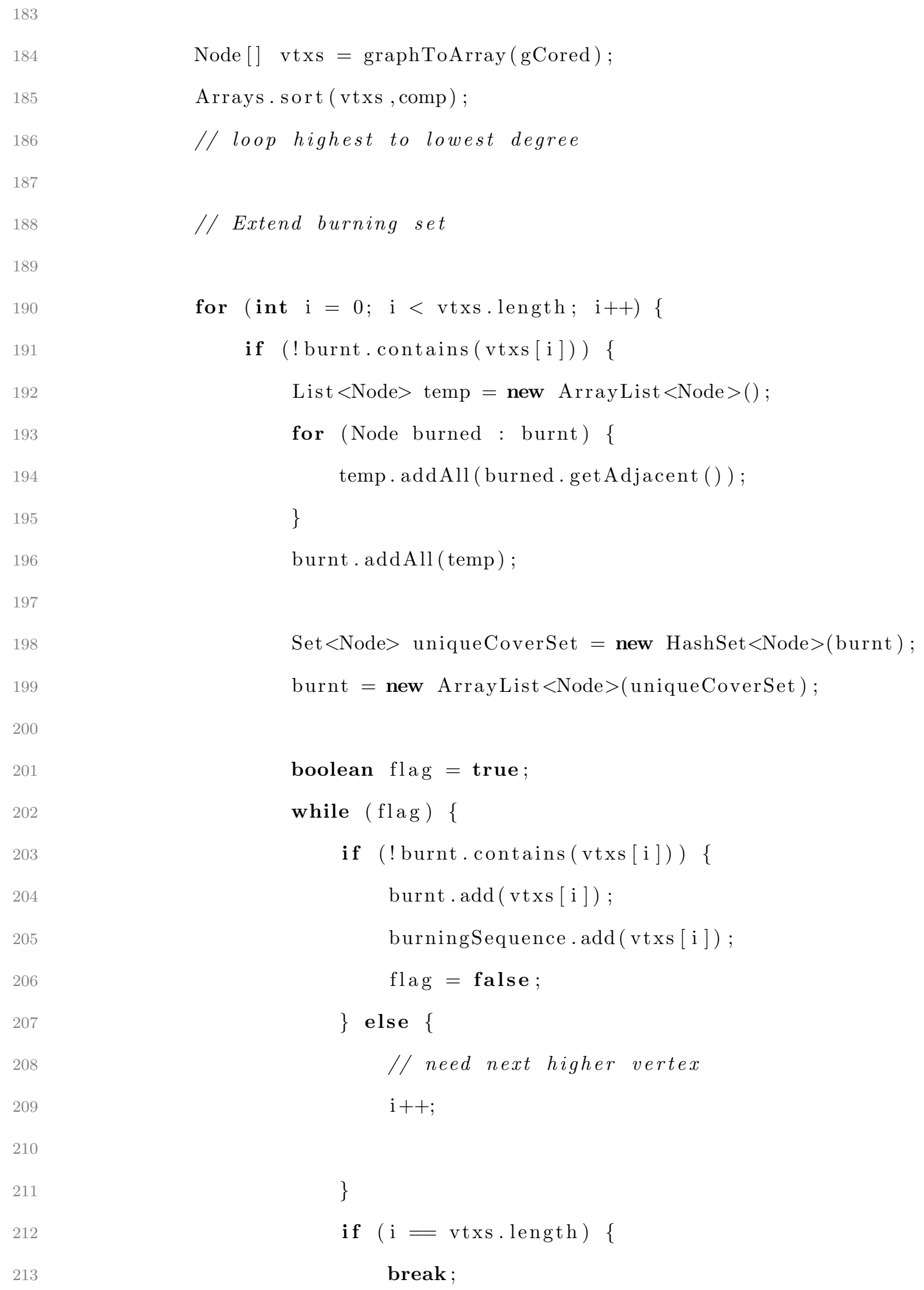

Node [] vtxs $=$ graphToArray $($ gCored $)$;

Arrays.sort (vtxs, comp);

// loop highest to lowest degree

// Extend burning set

for (int i $=0 ; \mathrm{i}<$ vtxs.length; i++) \{

if (! burnt. contains (vtxs [i])) \{

List $<$ Node $>$ temp $=$ new ArrayList $<$ Node $>()$;

for (Node burned : burnt) \{ temp.addAll (burned.getAdjacent ( )) ;

\}

burnt. addAll (temp);

Set $<$ Node $>$ uniqueCoverSet $=$ new HashSet $<$ Node $>$ (burnt $)$; burnt $=$ new ArrayList $<$ Node $>$ (uniqueCoverSet $)$;

boolean flag $=$ true;

while (flag) \{

if (!burnt.contains $(\operatorname{vtxs}[\mathrm{i}]))\{$

burnt.add $(\operatorname{vtxs}[\mathrm{i}])$;

burningSequence $\operatorname{add}(\operatorname{vtxs}[\mathrm{i}])$;

flag = false

\} else \{

// need next higher vertex

$\mathrm{i}++$;

\}

if $(\mathrm{i}=$ vtxs.length $)\{$

break ; 


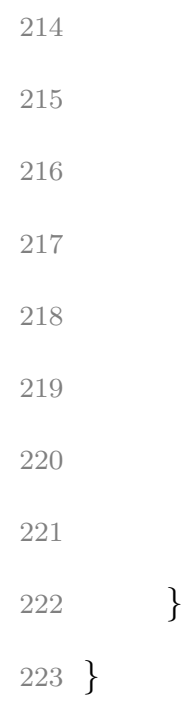

\section{MGEOP.java}

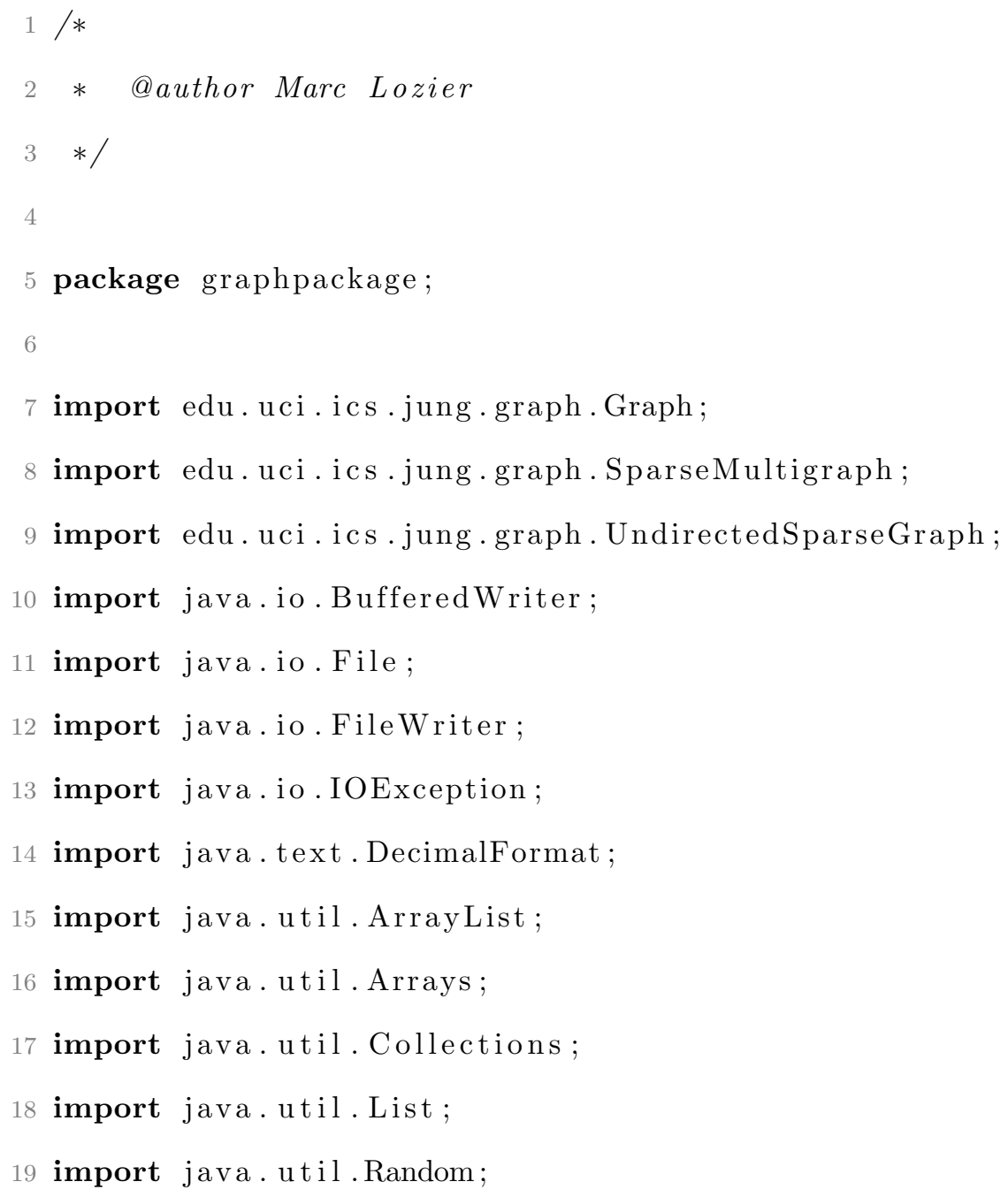




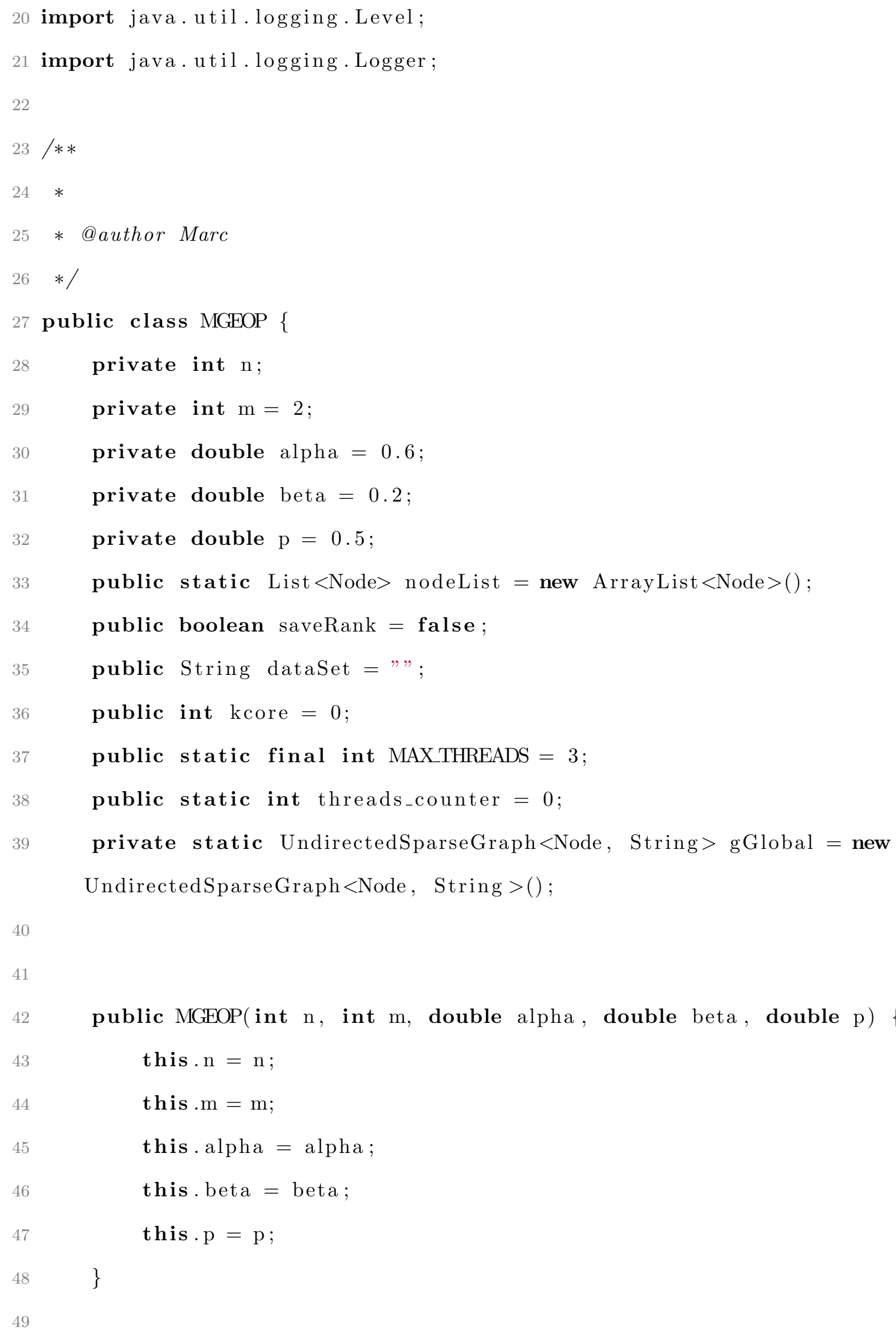


// INV-MGEO-P

public UndirectedSparseGraph $<$ Node, String $>$ generateInvGraph () \{ gGlobal $=$ new UndirectedSparseGraph $<$ Node, String $>()$;

createNodes ();

generateRank ( );

generateRadius ();

createEdgesInv ( ) ;

return gGlobal;

\}

// MGEQ-P

public UndirectedSparseGraph $<$ Node, String $>$ generateGraph ()\{

gGlobal $=$ new UndirectedSparseGraph $<$ Node, String $>()$;

createNodes ();

generateRank ( ) ;

generateRadius ( ) ;

createEdges ();

return gGlobal;

\}

public Graph $<$ Node, String $>$ generateGraphSpeedTest ()\{

System.out.println("> Creating Nodes");

createNodes ( ) ;

System.out.println("> Generating Rank");

generateRank ( ) ;

System.out.println("> Generating Radius");

generateRadius ( ) ;

System.out.println("> Creating Edges");

createEdgesThread ();

System.out.println("> Complete");

return gGlobal; 


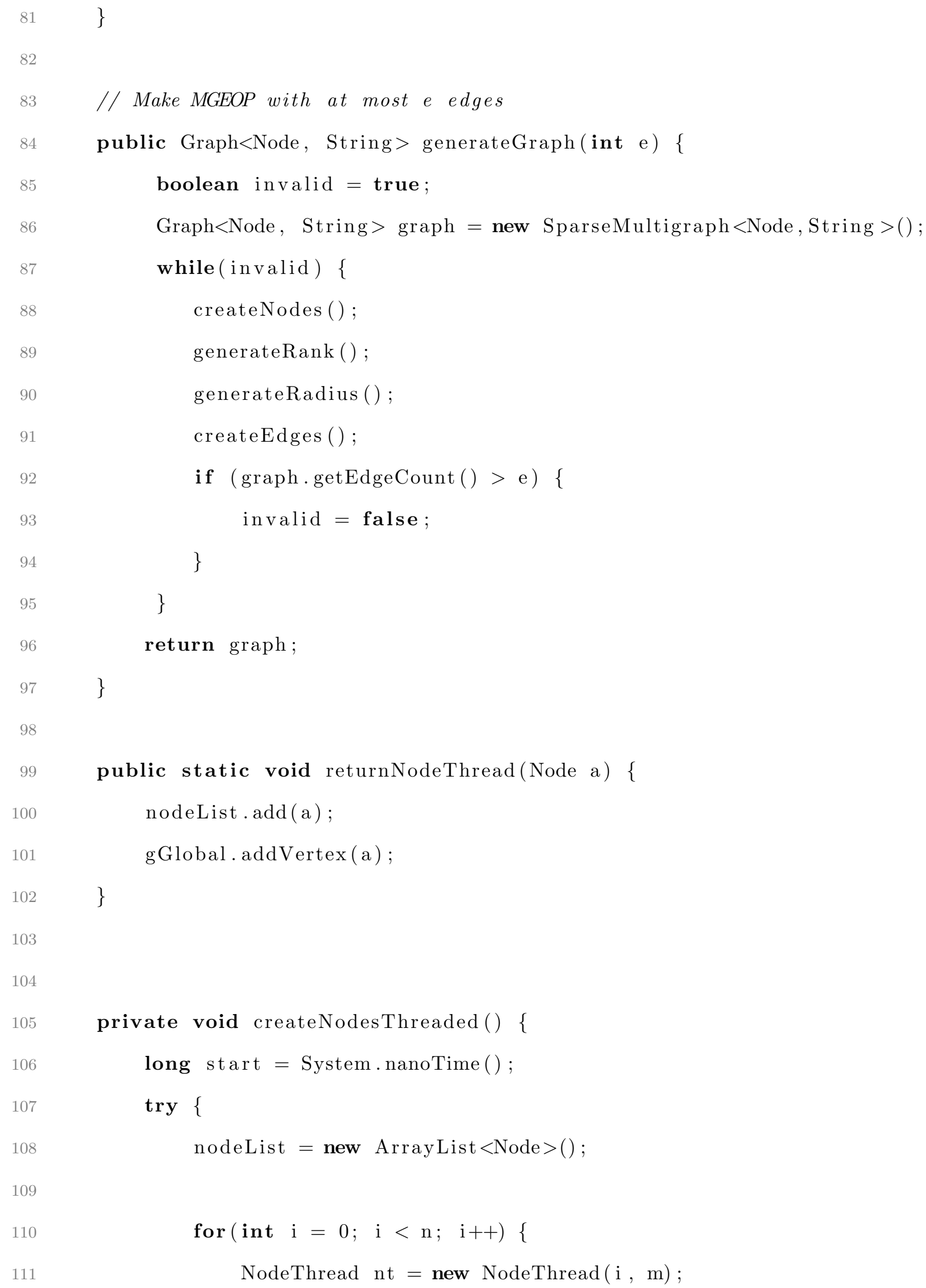


Thread $\mathrm{t}=$ new $\operatorname{Thread}(\mathrm{nt})$;

t. start ();

if $(\mathrm{i}=(\mathrm{n} / 4))\{$

System.out.println("25\% done Nodes");

t.join ();

\} else if $(\mathrm{i}=(\mathrm{n} / 2))\{$

System.out.println("50\% done Nodes");

t. join () ;

\} else if $(\mathrm{i}=(3 *(\mathrm{n} / 4)))\{$

System.out.println("75\% done Nodes");

t.join ();

\}

\section{\}}

\} catch (InterruptedException ex) \{

Logger . getLogger (MGEOP. class .getName()) . log (Level.SEVERE, null, ex ) ;

\}

long dur $=$ System.nanoTime ()$-$ start;

System .out.println (dur);

\}

private void createNodes () \{

long start $=$ System.nanoTime () ;

nodeList $=$ new ArrayList $<$ Node $>(n)$;

Node tmp;

for (int $\mathrm{i}=0 ; \mathrm{i}<\mathrm{n} ; \mathrm{i}++)\{$

tmp $=$ new $\operatorname{Node}(\mathrm{i}, \mathrm{m})$;

for $($ int temp $=0 ;$ temp $<\mathrm{m} ;$ temp ++$)\{$ tmp. setCoordinates (Math.random(), temp); 


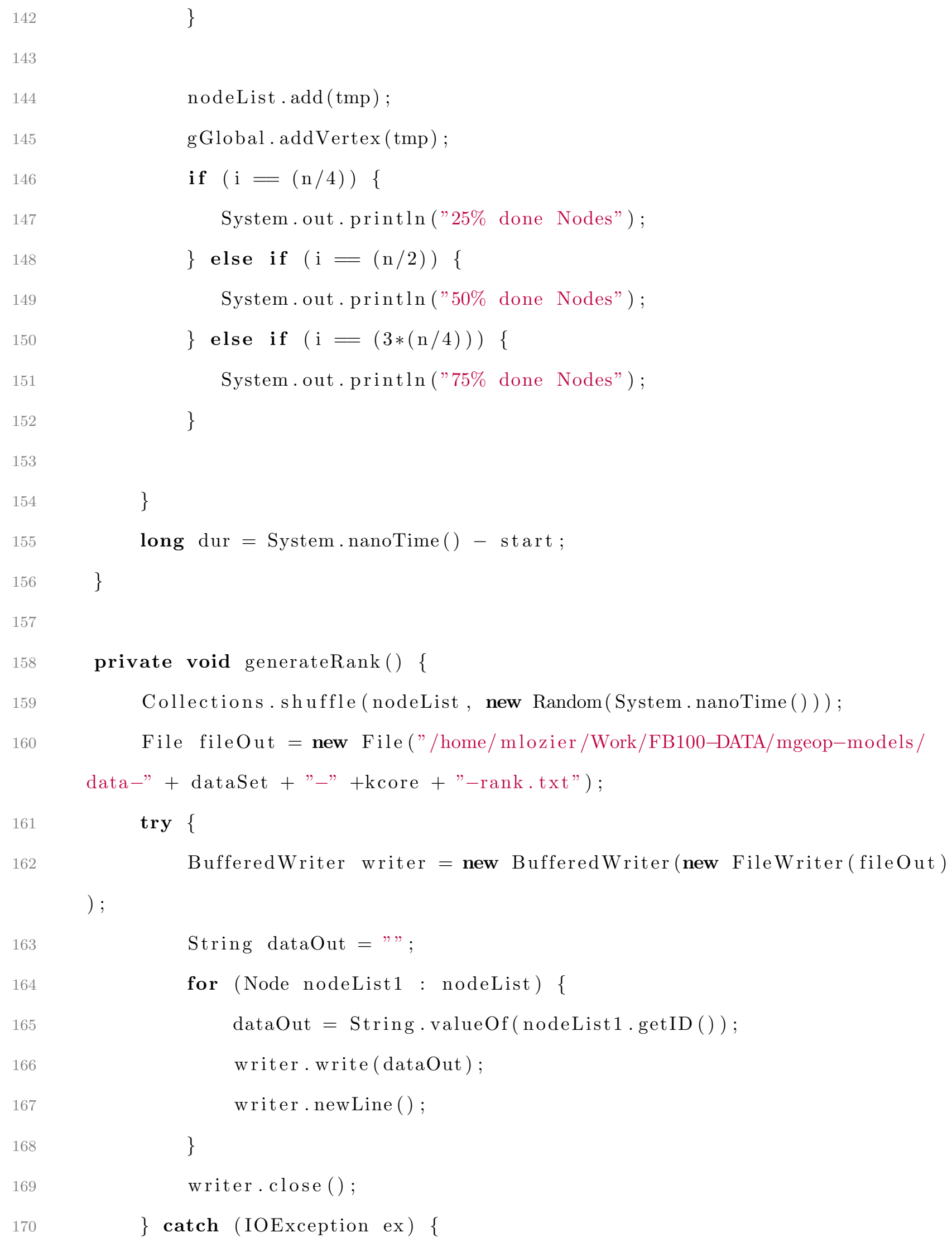

nodeList . add (tmp);

gGlobal . addVertex (tmp) ;

if $(\mathrm{i}=(\mathrm{n} / 4))\{$

System.out.println("25\% done Nodes");

\} else if $(\mathrm{i}=(\mathrm{n} / 2))\{$

System.out.println ("50\% done Nodes");

\} else if $(\mathrm{i}=(3 *(\mathrm{n} / 4)))\{$

System.out.println ("75\% done Nodes");

\}

\}

long dur = System.nanoTime ()$-$ start;

\}

private void generateRank () \{

Collections.shuffle (nodeList, new Random(System.nanoTime ()) );

File fileOut = new File("/home/mlozier/Work/FB100-DATA/mgeop-models/ data-" + dataSet + "-" +kcore + "-rank.txt");

$\operatorname{try}\{$

BufferedWriter writer = new BufferedWriter(new FileWriter(fileOut) )

String dataOut $="$, ;

for (Node nodeList 1 : nodeList) \{

dataOut $=$ String $\cdot$ valueOf $($ nodeList $1 \cdot \operatorname{getID}())$;

writer. write (dataOut);

writer newLine ( ) ;

\}

writer.close ();

\} $\operatorname{catch}($ IOException ex $)\{$ 

ex);

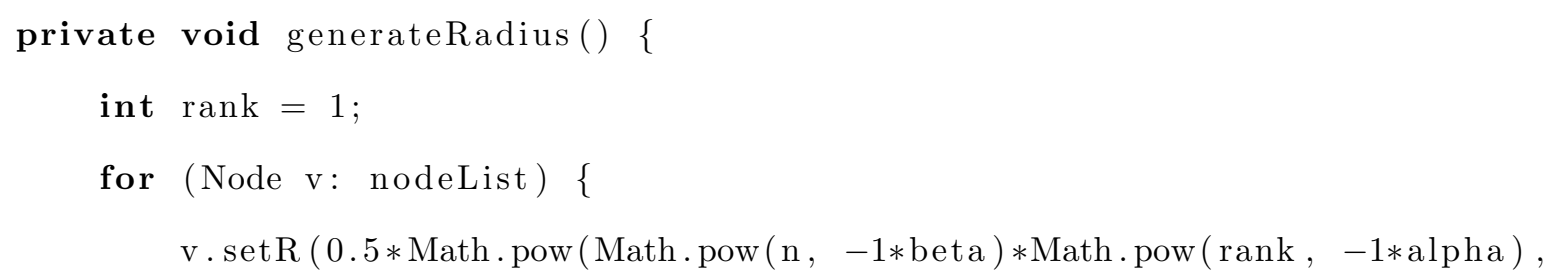

y) ; 


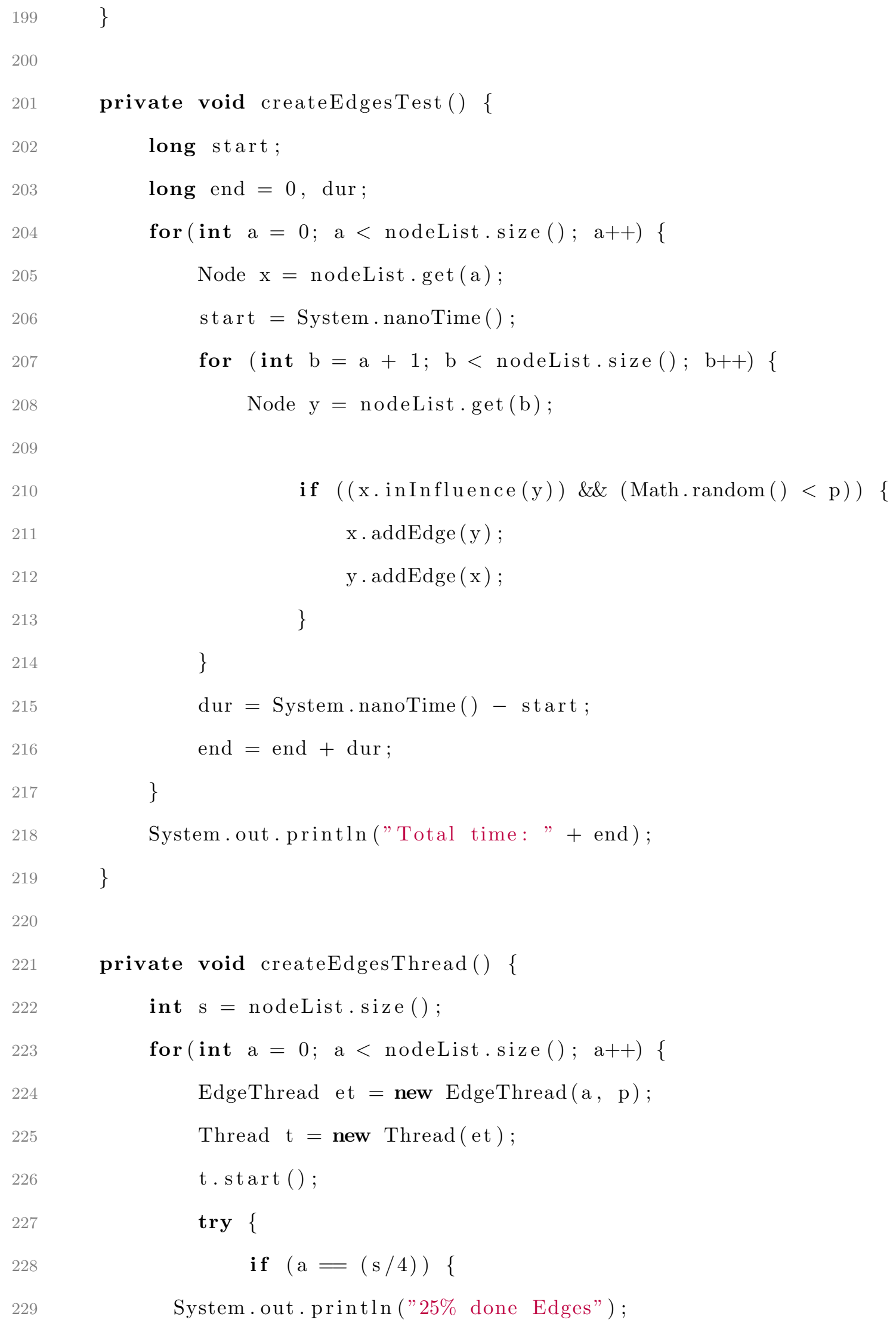




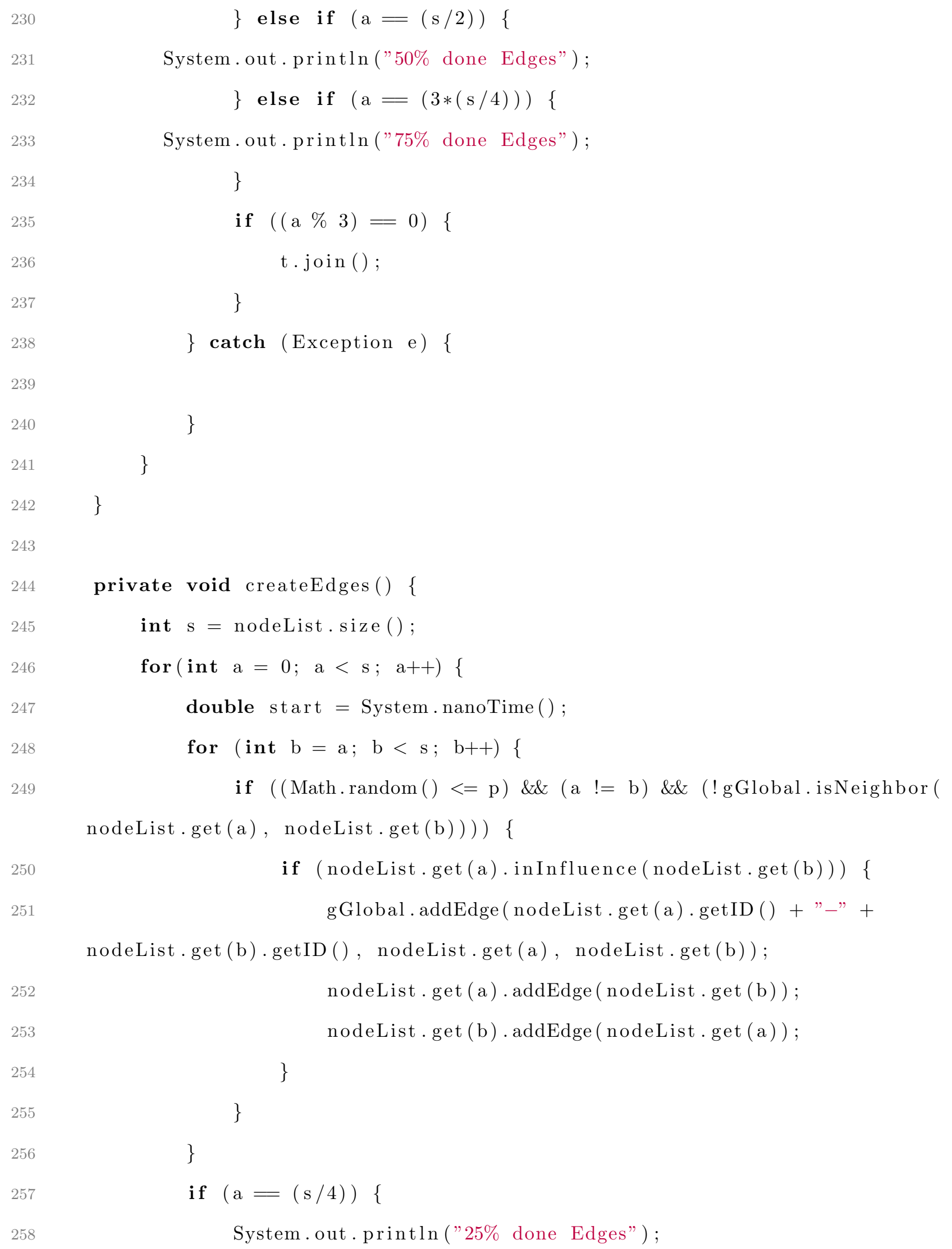




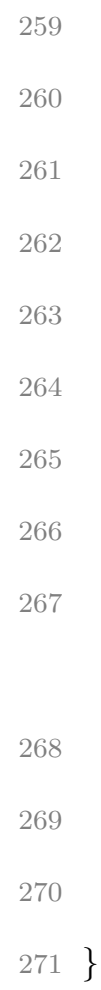

\section{RandomGraphGenerator.java}

1 package graphpackage;

2

3 import edu.uci.ics.jung.graph.Graph;

4 import edu.uci.ics.jung.graph.SparseMultigraph;

5 import edu.uci.ics.jung.graph. UndirectedSparseGraph;

6 import java.text. DecimalFormat;

7 import java.util. ArrayList;

8 import java.util. Arrays;

9 import java.util. Comparator;

10 import java.util. List;

import java.util. concurrent. TimeUnit;

import javafx.scene.shape.Box;

import matlabcontrol.*;

14

15 public class RandomGraphGenerator \{ 
public static Box box;

public RandomGraphGenerator(int $n$, double p) \{

this.n $=\mathrm{n}$;

this $\cdot \mathrm{p}=\mathrm{p}$;

\}

public UndirectedSparseGraph $<$ Node, String $>$ generateGNPRandomGraph() \{ UndirectedSparseGraph $<$ Node, String $>$ g $=$ new UndirectedSparseGraph $<$ Node , String $>()$;

List $<$ Node $>$ nodeList $=$ new ArrayList $<$ Node $>()$;

System . out . println ("Creating $G(n, p)=G("+n+", "+p+") ")$;

for $($ int $\mathrm{i}=0 ; \mathrm{i}<\mathrm{n} ; \mathrm{i}++)\{$

Node $\operatorname{tmp}=$ new Node $(\mathrm{i})$;

g. addVertex (tmp);

nodeList . add (tmp);

\}

for $($ int $\mathrm{i}=0 ; \mathrm{i}<\mathrm{n} ; \mathrm{i}++)\{$

Node $\mathrm{a}=\operatorname{nodeList} \cdot \operatorname{get}(\mathrm{i})$;

for (int $\mathrm{j}=\mathrm{i}+1 ; \mathrm{j}<\mathrm{n} ; \mathrm{j}++)\{$

Node $\mathrm{b}=$ nodeList. $\operatorname{get}(\mathrm{j})$;

if $($ Math $\cdot \operatorname{random}()<\mathrm{p})\{$

g.addEdge (a.getID ()$+"-$ to-" + b $\cdot \operatorname{getID}()$, a, b );

a . addEdge (b);

// NOTE: this line below is not needed (compresses file if

omitted)

b.addEdge (a); 


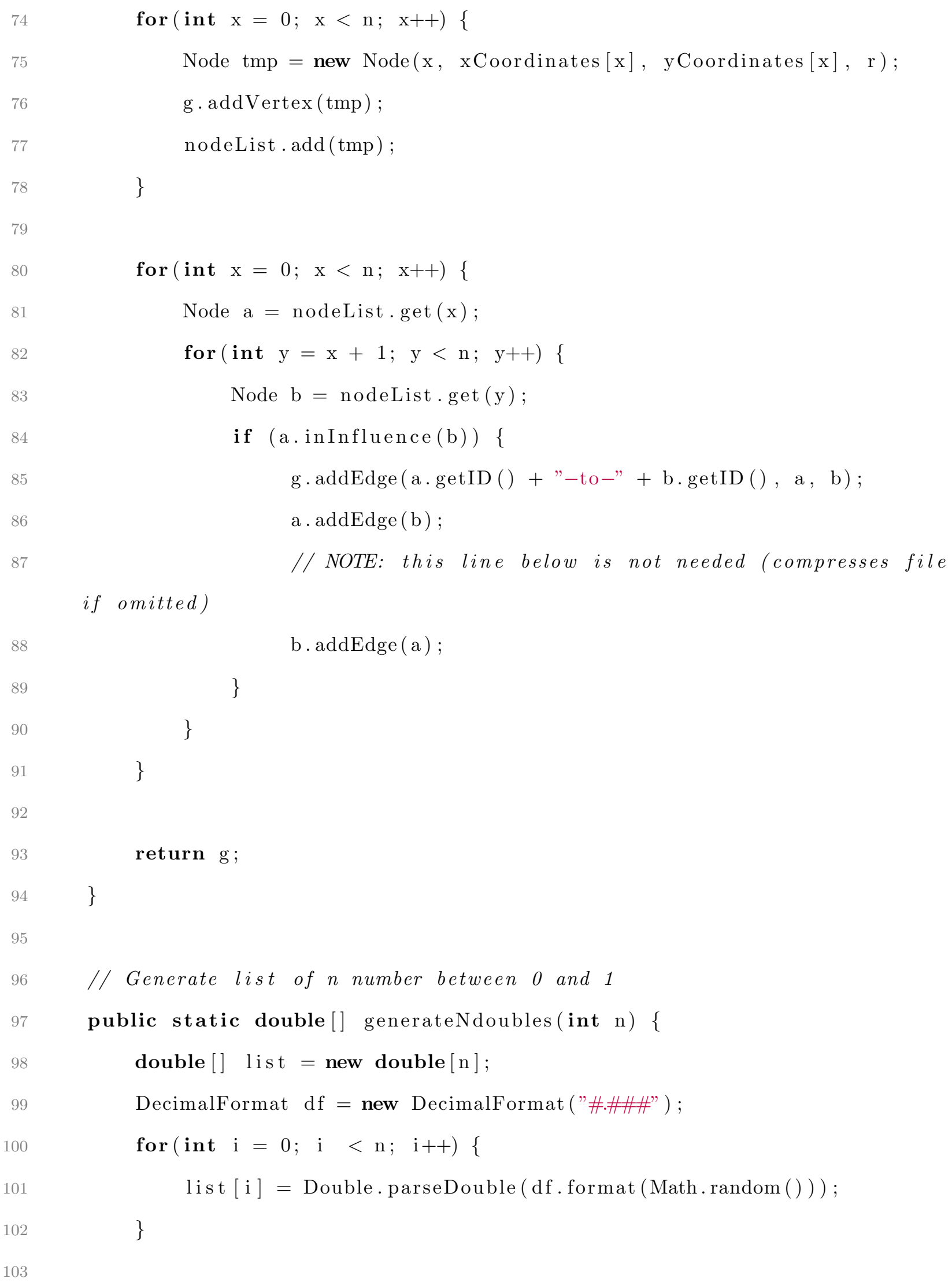

for $($ int $\mathrm{x}=0 ; \mathrm{x}<\mathrm{n} ; \mathrm{x}++)\{$

Node $\operatorname{tmp}=$ new $\operatorname{Node}(\mathrm{x}, \mathrm{x}$ Coordinates $[\mathrm{x}], \mathrm{yCoordinates}[\mathrm{x}], \mathrm{r})$; g. addVertex (tmp); nodeList . add (tmp); \}

for $($ int $\mathrm{x}=0 ; \mathrm{x}<\mathrm{n} ; \mathrm{x}++)\{$

Node $\mathrm{a}=$ nodeList.get $(\mathrm{x})$;

for $($ int $\mathrm{y}=\mathrm{x}+1 ; \mathrm{y}<\mathrm{n} ; \mathrm{y}++)\{$

Node $\mathrm{b}=$ nodeList.get $(\mathrm{y})$;

if (a.inInfluence (b)) \{ $\mathrm{g} \cdot \operatorname{addEdge}(\mathrm{a} \cdot \operatorname{getID}()+"-\mathrm{to}-"+\mathrm{b} \cdot \operatorname{getID}(), \mathrm{a}, \mathrm{b})$;

a . addEdge (b) ;

// NOTE: this line below is not needed (compresses file

if omitted)

8


return list;

$105\}$

106

$107\}$ 


\section{B.2. SNAP Code $(\mathrm{C}++)$}

graphgenerator.cpp

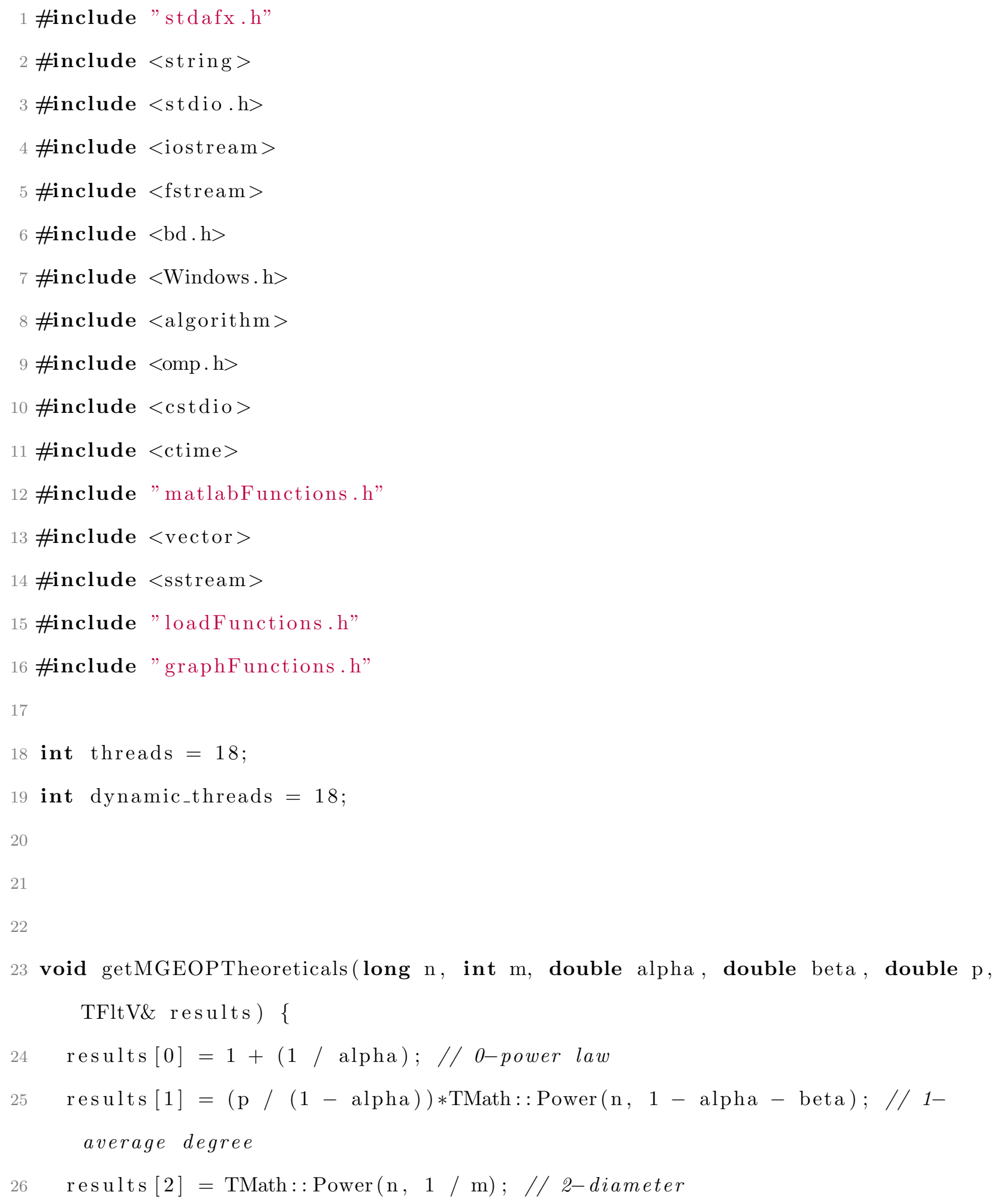




\section{\}}

// $x$ - degrees | $y$ - number of vertices

TVec $<$ TIntPr $>$ degreeCount ;

\{

bool exist $=$ false

degreeCount [tmp ]. Val2++;

exist $=$ true

\}

\}

if (! exist) \{

degreeCount . Add ( TIntPr(NI.GetDeg ( ), 1));

\}

\}

int $\mathrm{n}=$ degreeCount.Len ();

double b_max $=0$;

double s_max $=0$;

double e_max $=\mathrm{n}$;

double sum $=0.0 ;$

double sum $2=0.0 ;$

double sum $3=0.0$;

double sum $4=0.0$ results $[3]=$ TMath: $: \log ($ TMath $:: \log (\mathrm{n})) ; / / 3$-average distance

for (PUNGraph: : TObj : : TNodeI NI $=$ Graph->BegNI ()$;$ NI $<$ Graph - EndNI ()$;$ NI++)

for (int $\operatorname{tmp}=0 ; \operatorname{tmp}<$ degreeCount. Len ()$; \operatorname{tmp}++)\{$

if ( degreeCount $[\operatorname{tmp}]$. Val1 $=$ NI. GetDeg ()$)\{$

for $($ double incre $=0 ;$ incre $<0.5 ;$ incre $=$ incre +0.001$)\{$ 
double $\mathrm{s}=$ incre $* \mathrm{n}$;

double $\mathrm{e}=\mathrm{n}$;

for $($ int $\operatorname{tmp}=\operatorname{int}(\mathrm{s}) ; \operatorname{tmp}<\operatorname{int}(\mathrm{e}) ; \operatorname{tmp}++)\{$

int $\mathrm{x}=$ degreeCount $[\mathrm{tmp}]$. Val1;

int $\mathrm{y}=$ degreeCount $[\mathrm{tmp}]$. Val2;

sum $+=$ TMath $:: \log (\mathrm{x}) *$ TMath $:: \log (\mathrm{y}) ;$

sum2 += TMath: : $\log (\mathrm{x})$;

sum3 += TMath: : $\log (\mathrm{y})$;

sum4 += TMath: : $\log (\mathrm{x}) *$ TMath $:: \log (\mathrm{x})$;

\}

int $\mathrm{n} 2=\operatorname{int}(\mathrm{e})-\operatorname{int}(\mathrm{s})$;

double $\mathrm{b}=((\mathrm{n} 2 * \operatorname{sum})-(\operatorname{sum} 2 * \operatorname{sum} 3)) /((\mathrm{n} 2 * \operatorname{sum} 4)-(\operatorname{sum} 2 * \operatorname{sum} 2))$;

if $($ b_max $>$ b) \{

$\mathrm{b} \_\max =\mathrm{b}$;

$\mathrm{s} \_\max =\mathrm{s} ;$

\}

\}

for $($ double incre $=0 ;$ incre $<0.5 ;$ incre $=$ incre +0.001$)\{$

double sum $=0.0$;

double $\operatorname{sum} 2=0.0 ;$

double $\operatorname{sum} 3=0.0 ;$

double $\operatorname{sum} 4=0.0 ;$

double $\mathrm{s}=\mathrm{s} \_$max;

double $\mathrm{e}=(1-$ incre $) * \mathrm{n}$;

for $($ int $\operatorname{tmp}=\operatorname{int}(\mathrm{s}) ; \operatorname{tmp}<\operatorname{int}(\mathrm{e}) ; \operatorname{tmp}++)\{$

int $\mathrm{x}=$ degreeCount $[\mathrm{tmp}]$. Val1;

int $\mathrm{y}=$ degreeCount $[\mathrm{tmp}]$. Val2;

sum $+=$ TMath $:: \log (\mathrm{x}) *$ TMath $:: \log (\mathrm{y})$;

sum2 += TMath: : $\log (\mathrm{x})$; 


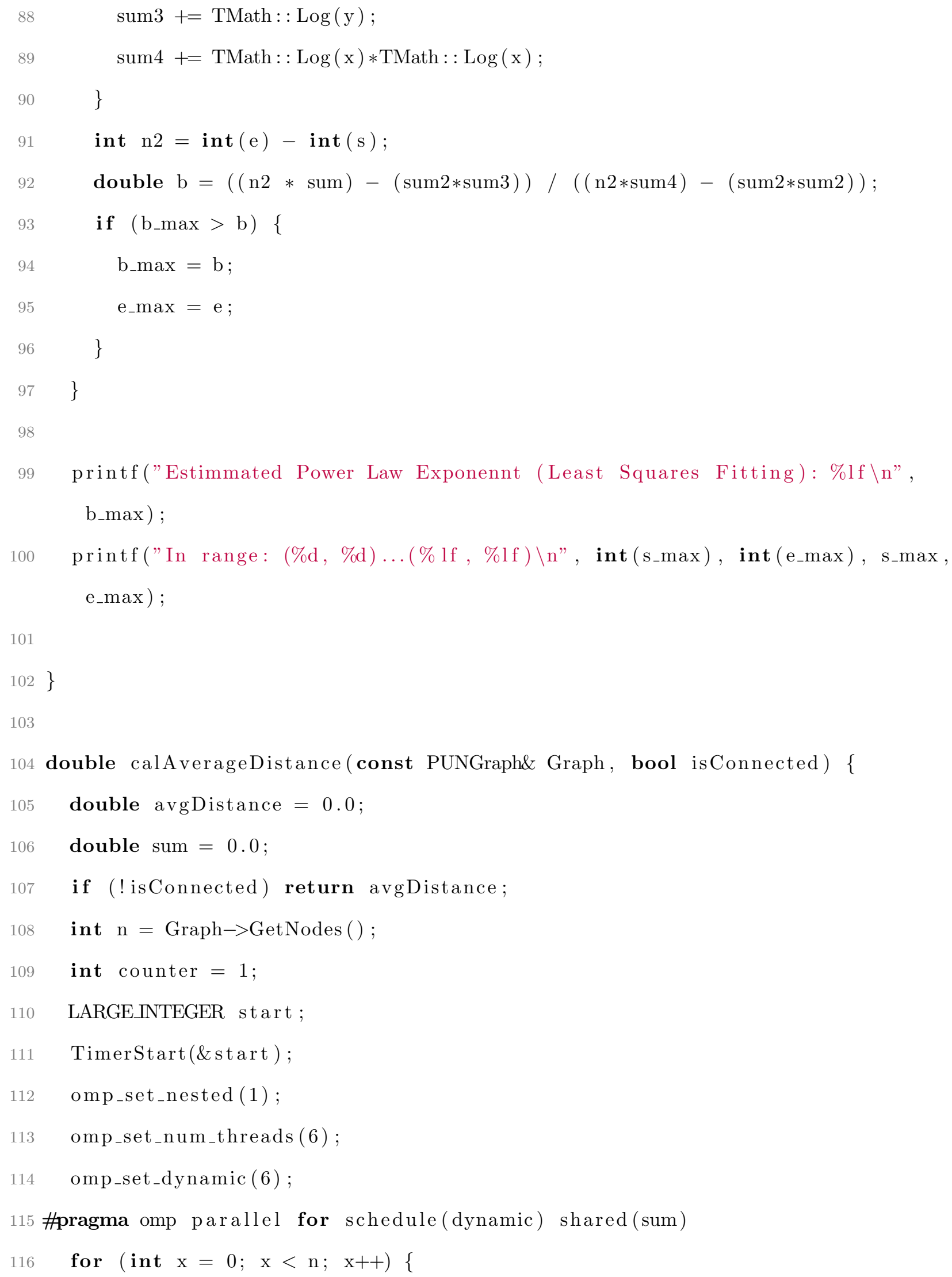




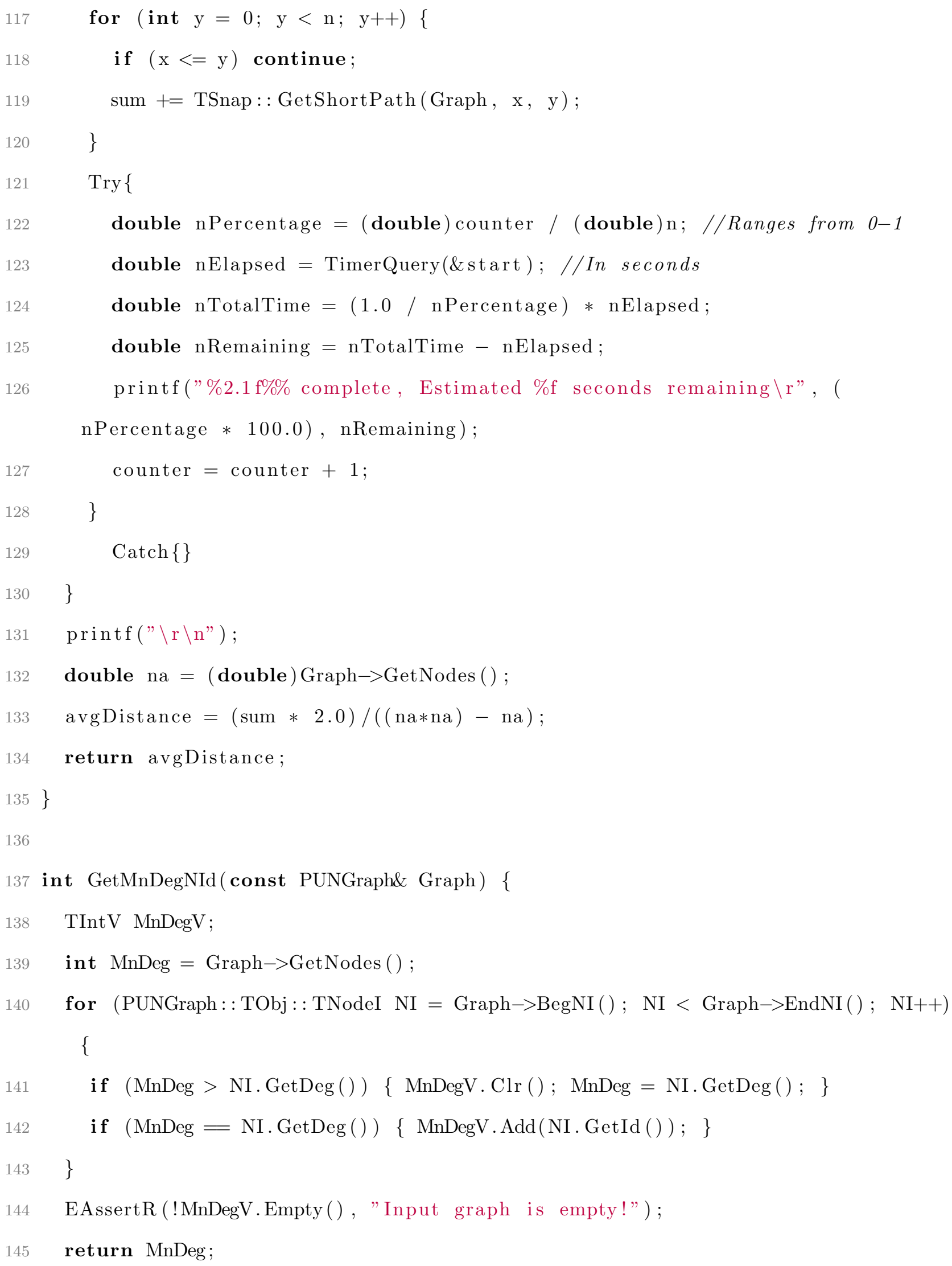

for (int $\mathrm{y}=0 ; \mathrm{y}<\mathrm{n} ; \mathrm{y}++)\{$

if $(\mathrm{x}<=\mathrm{y})$ continue; sum $+=$ TSnap : : GetShortPath (Graph, x, y) ;

\}

$\operatorname{Try}\{$

double nPercentage $=($ double $)$ counter $/$ (double $) \mathrm{n} ; / /$ Ranges from $0-1$ double nElapsed $=$ TimerQuery(\&start) $/ /$ In seconds double nTotalTime $=(1.0 /$ nPercentage $) *$ nElapsed;

double nRemaining $=$ nTotalTime - nElapsed; printf ( $\% 2.1 \mathrm{f} \% \%$ complete, Estimated \%f seconds remaining $\backslash \mathrm{r} "$, ( nPercentage *100.0), nRemaining); counter $=$ counter +1 ;

\}

$\operatorname{Catch}\{\}$ \} printf $(" \backslash \mathrm{r} \backslash \mathrm{n} ")$;

double na $=($ double $)$ Graph $\rightarrow$ GetNodes ()$;$ $\operatorname{avgDistance}=(\operatorname{sum} * 2.0) /(($ na $*$ na $)-$ na $) ;$

return avgDistance;

\}

int GetMnDegNId (const PUNGraph\& Graph) \{

TIntV MnDegV;

int $\mathrm{MnDeg}=$ Graph $\rightarrow$ GetNodes () ;

for (PUNGraph:: TObj:: TNodeI NI = Graph $\rightarrow$ BegNI ()$;$ NI $<$ Graph $\rightarrow$ EndNI ()$;$ NI++)

\{

if $(\operatorname{MnDeg}>$ NI.GetDeg ()$) \quad\{\operatorname{MnDegV} \cdot \operatorname{Clr}() ; \operatorname{MnDeg}=$ NI.GetDeg ()$;\}$

if $(\operatorname{MnDeg}=\mathrm{NI} \cdot \operatorname{GetDeg}())\{\operatorname{MnDegV} \cdot \operatorname{Add}(\mathrm{NI} \cdot \operatorname{GetId}()) ;\}$

\}

EAssertR (!MnDegV.Empty (), "Input graph is empty!"); return $\mathrm{MnDeg}$; 


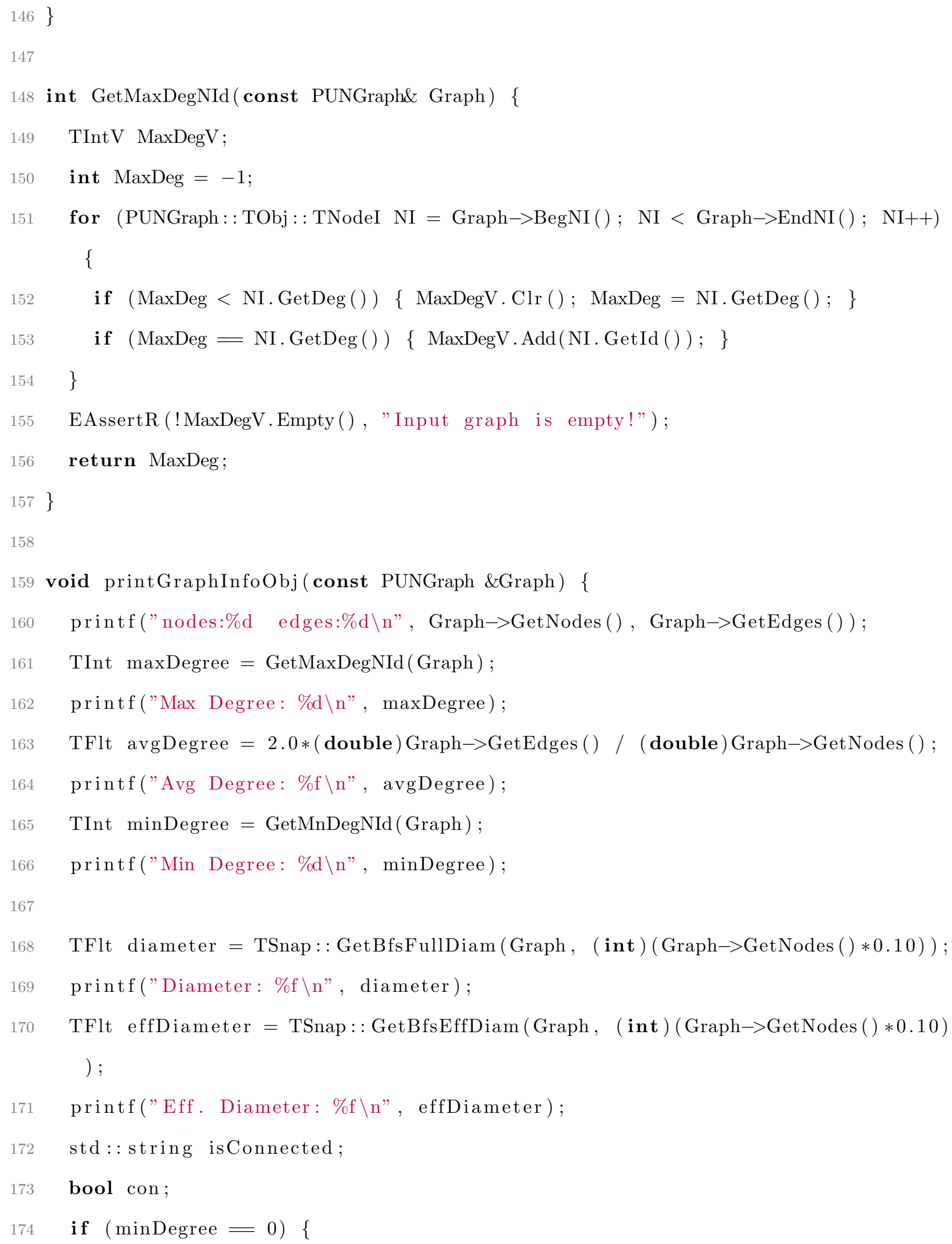




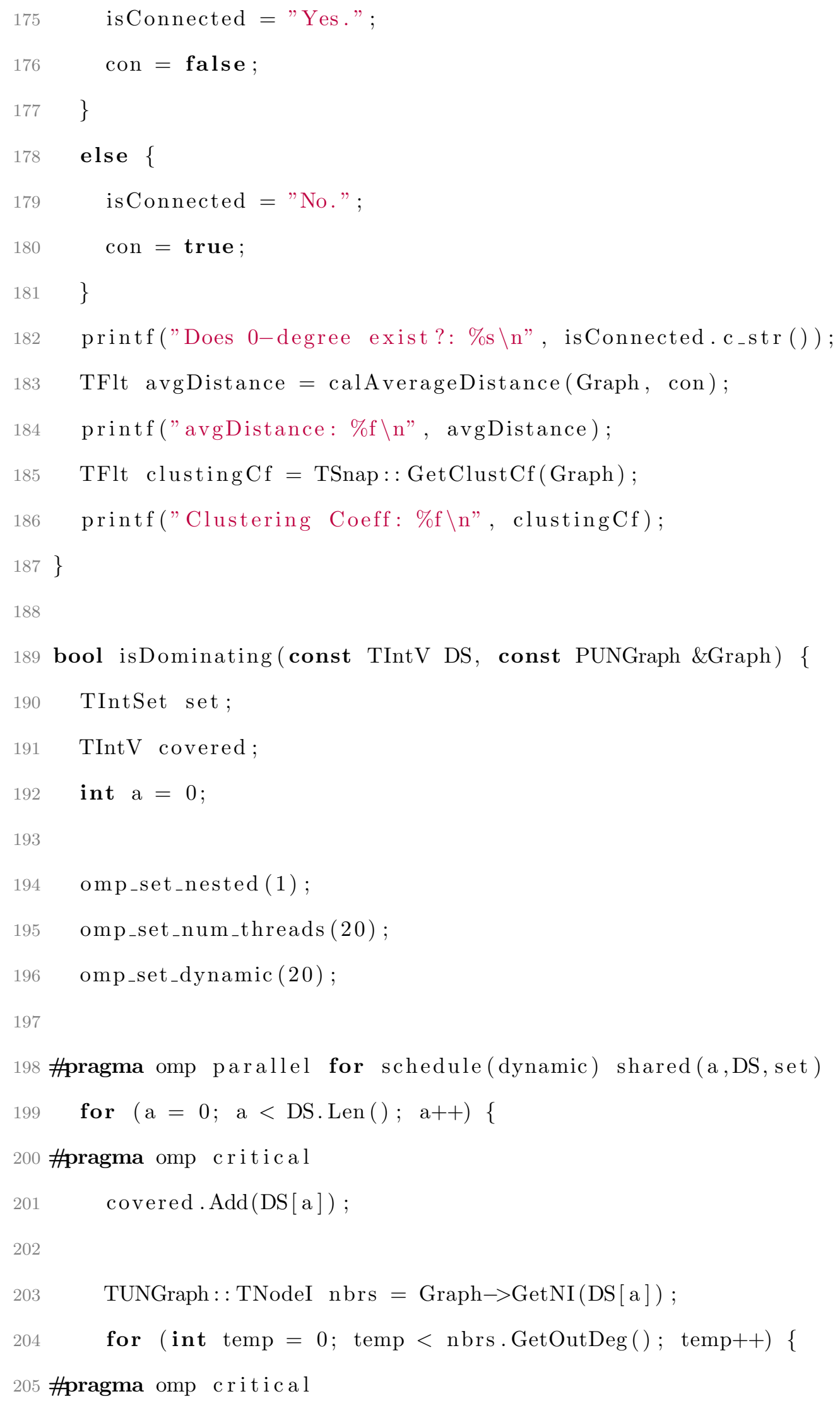




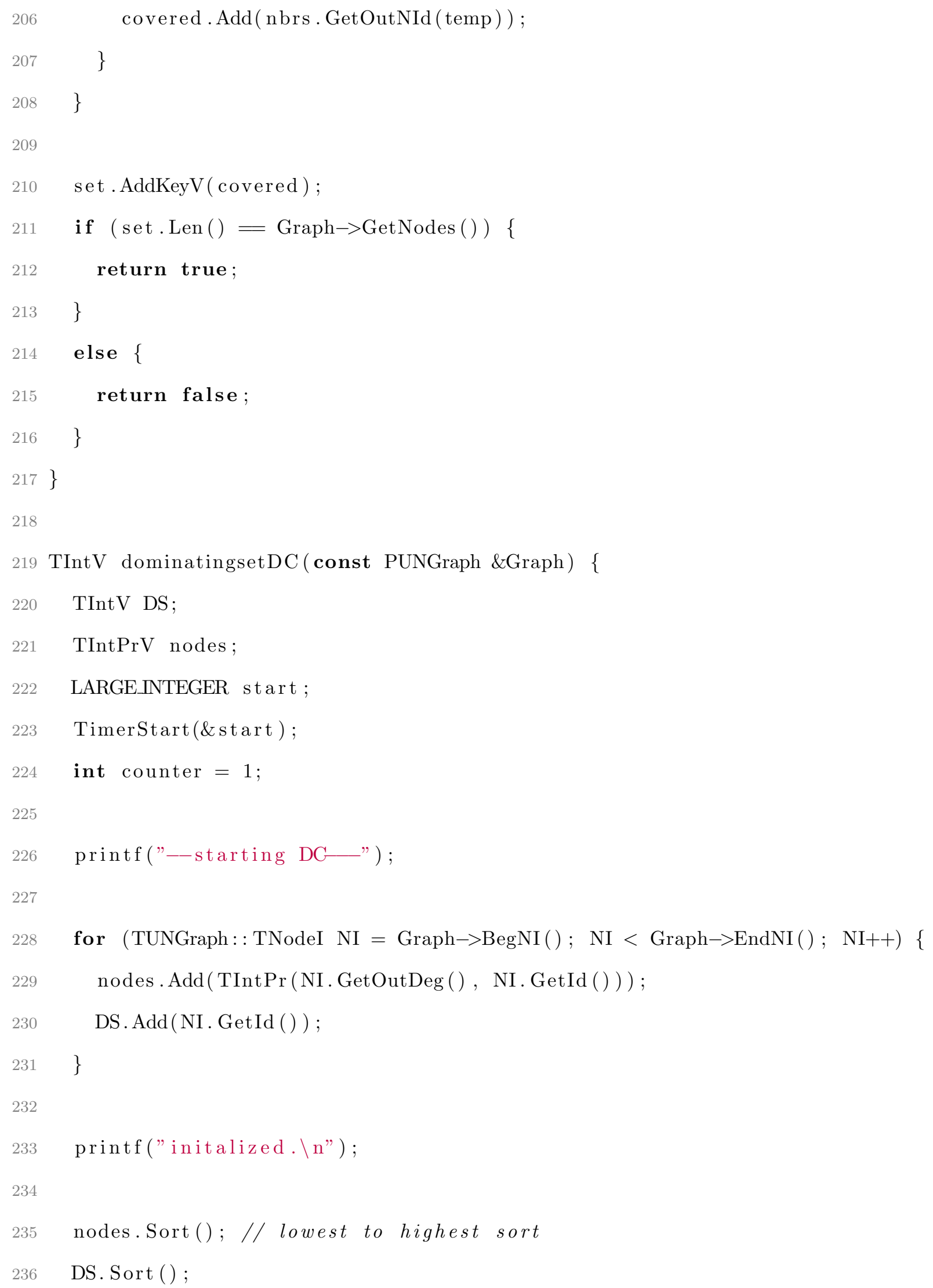




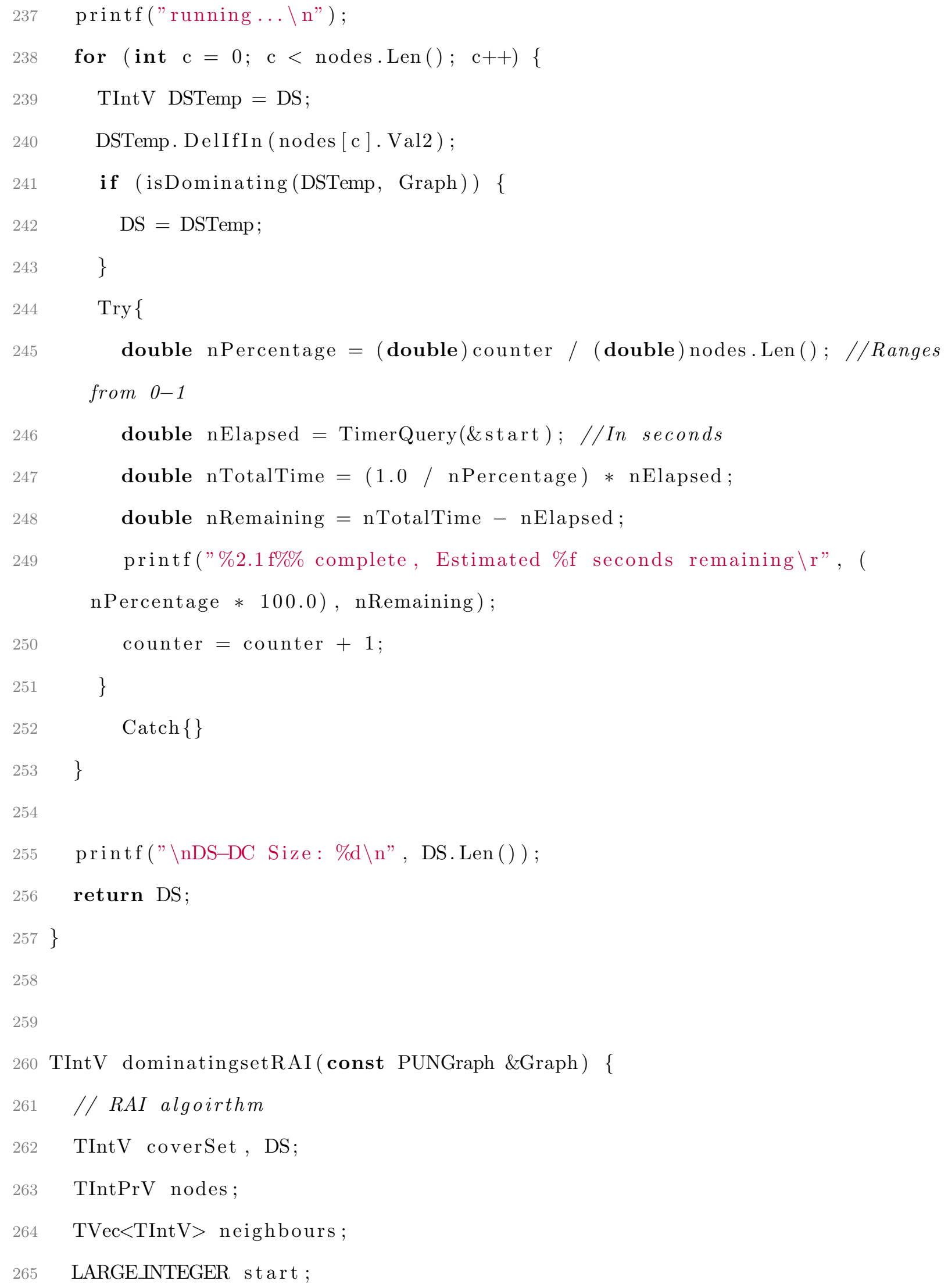




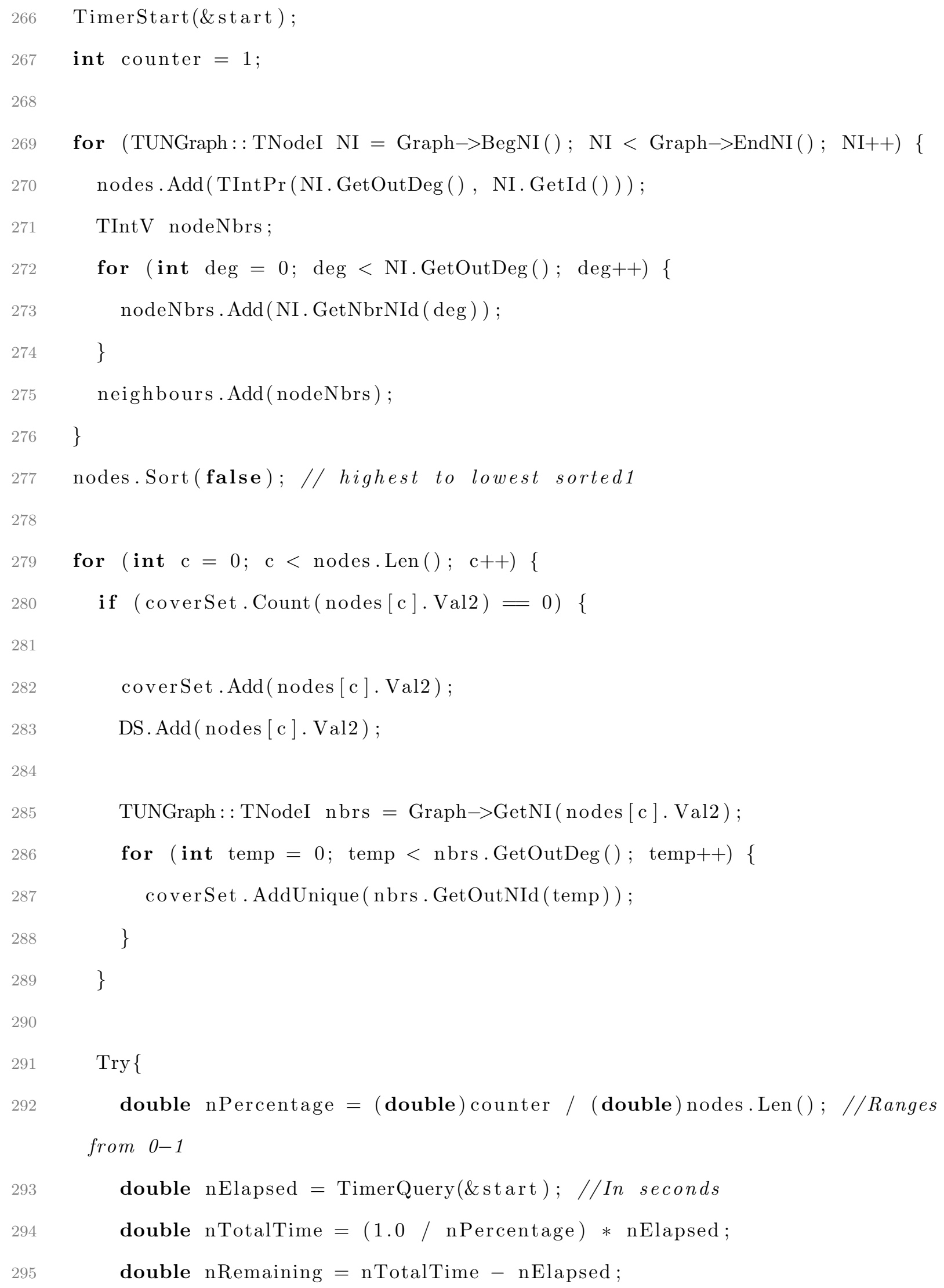

for (TUNGraph: : TNodeI NI = Graph->BegNI () ; NI < Graph $->$ EndNI ()$;$ NI++) \{ nodes.Add (TIntPr (NI.GetOutDeg (), NI.GetId ( ) ) ); TIntV nodeNbrs;

double nElapsed $=$ TimerQuery(\&start); //In seconds

double nTotalTime $=(1.0 /$ nPercentage $) *$ nElapsed;

double nRemaining $=$ nTotalTime - nElapsed; 


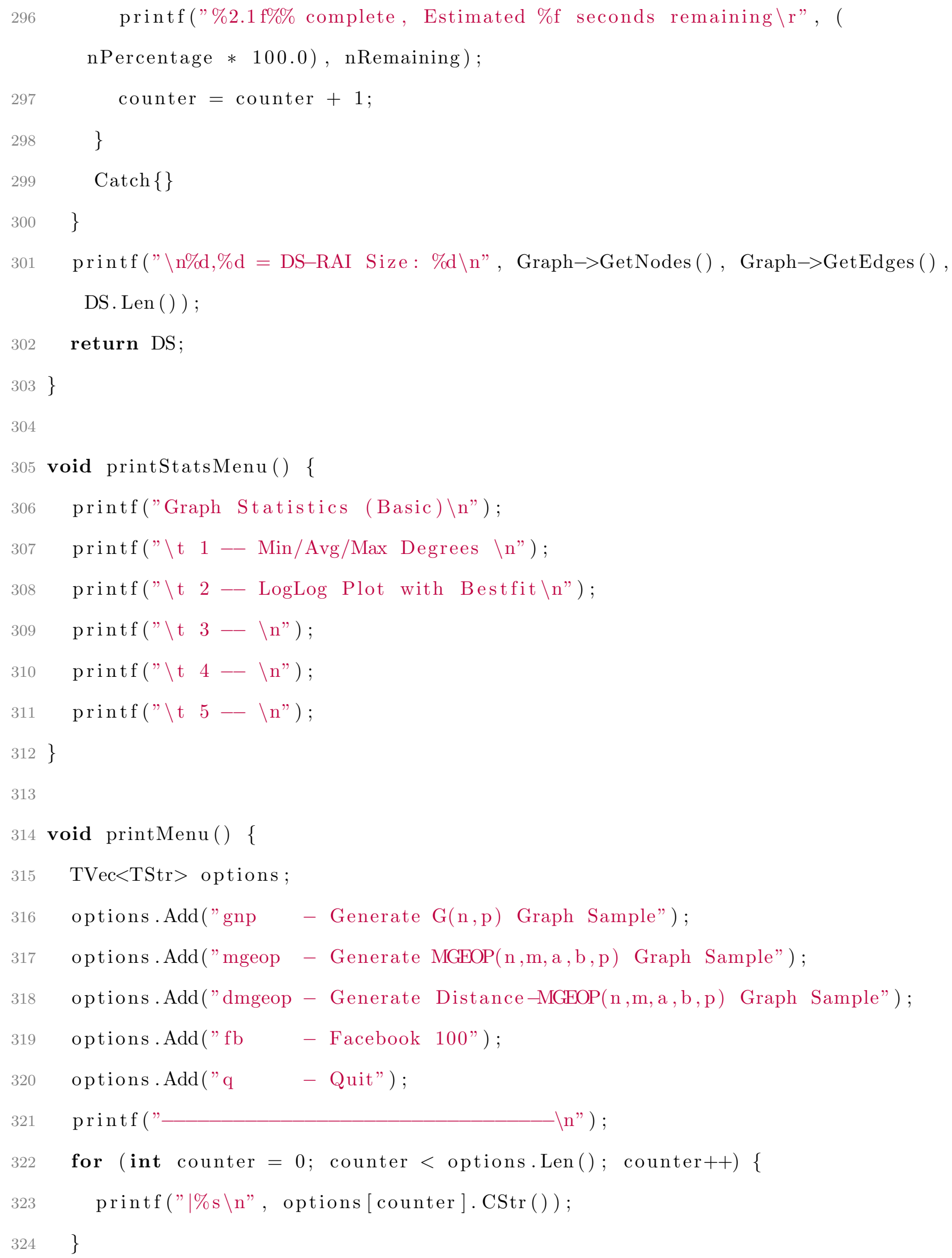




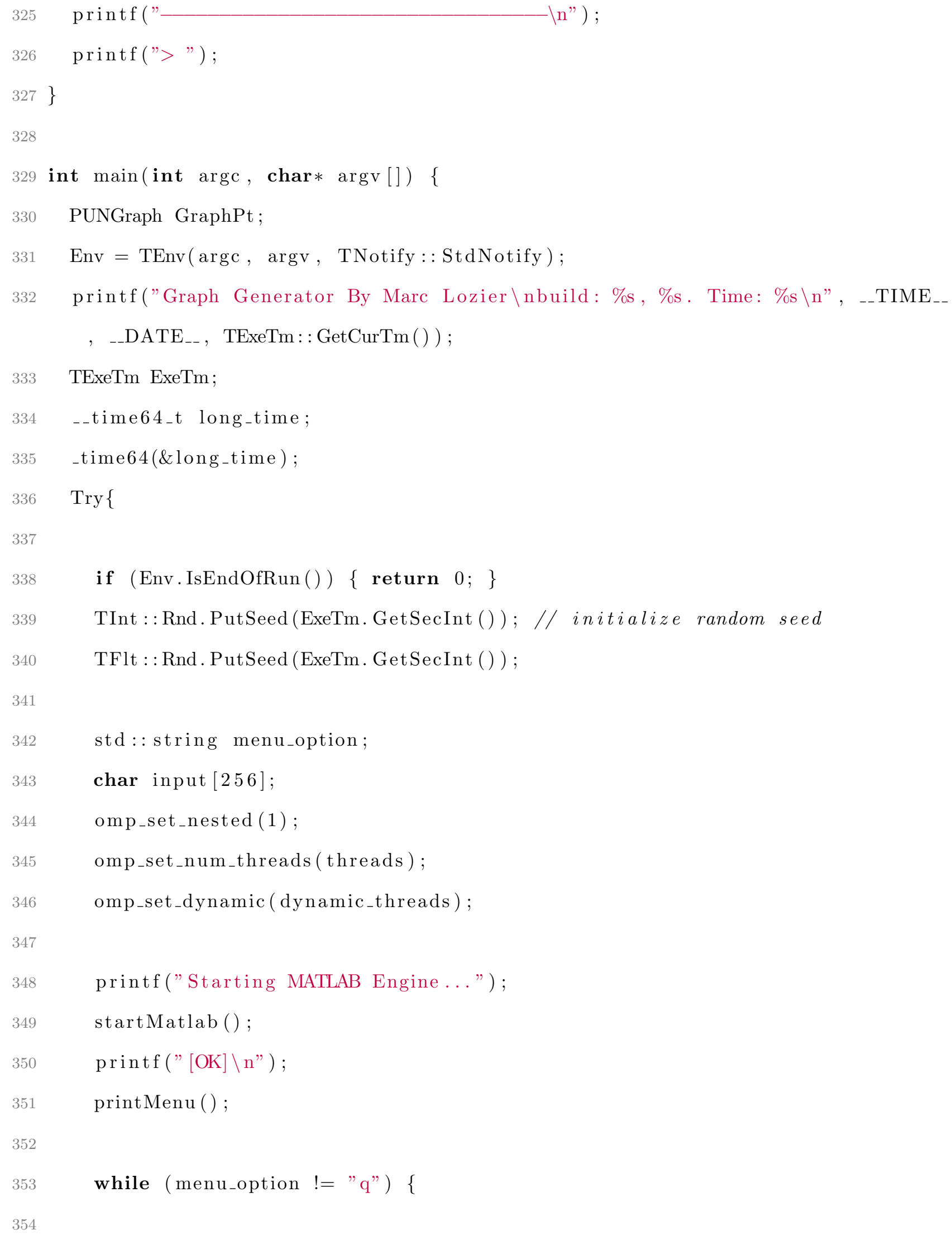




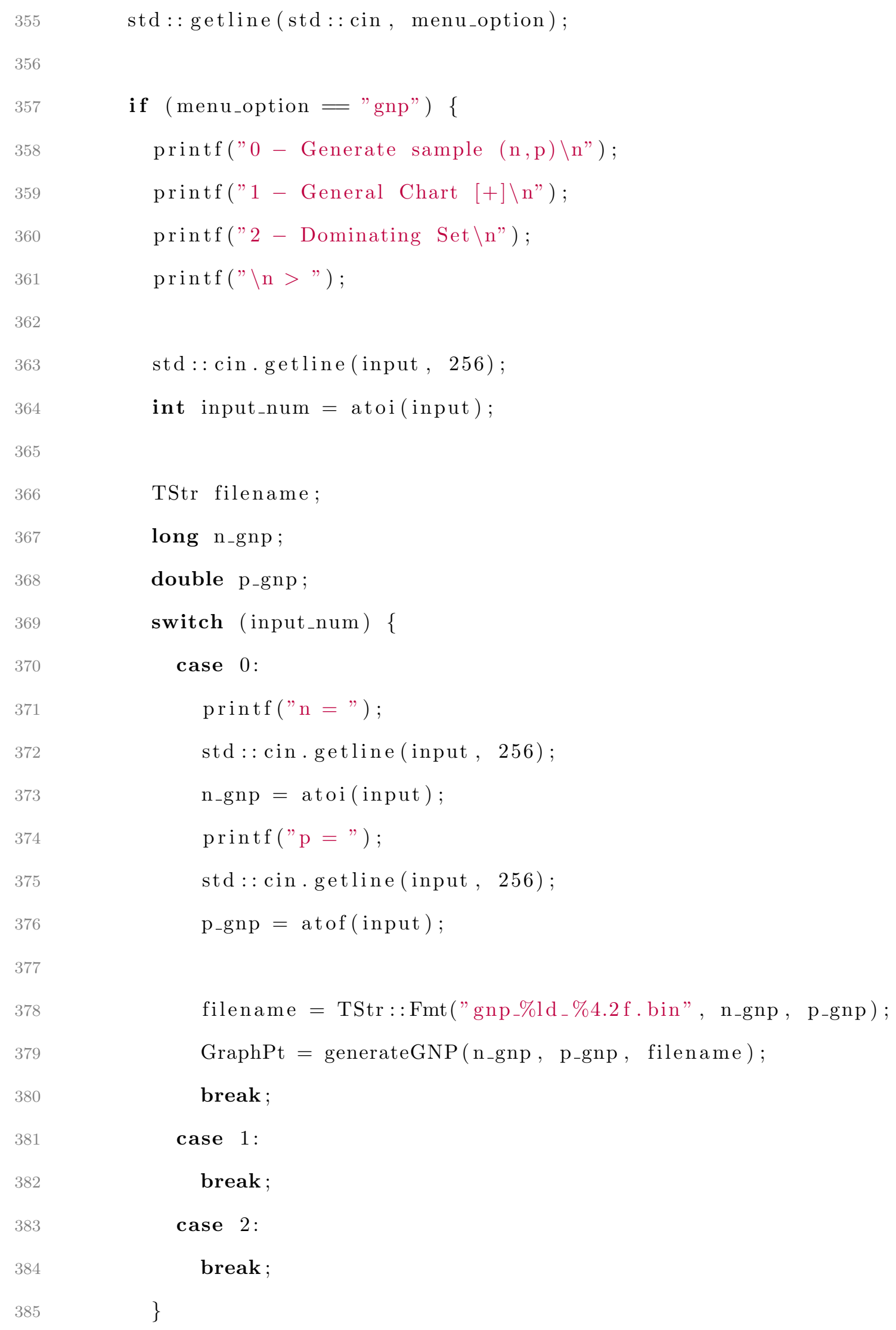




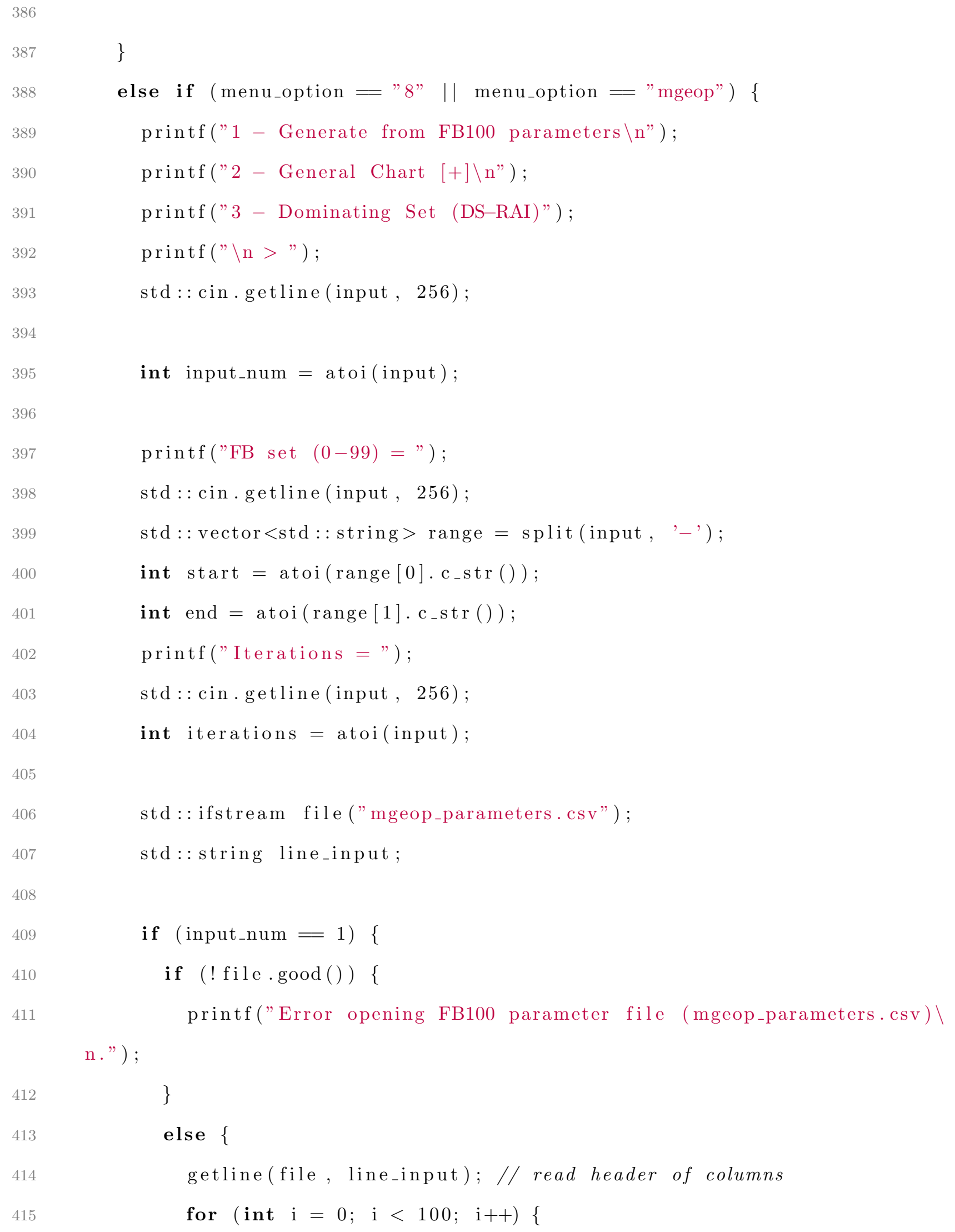

else if (menu_option $=" 8 "||$ menu_option $=="$ mgeop" $)\{$ printf("1 - Generate from FB100 parameters $\backslash n ")$; 


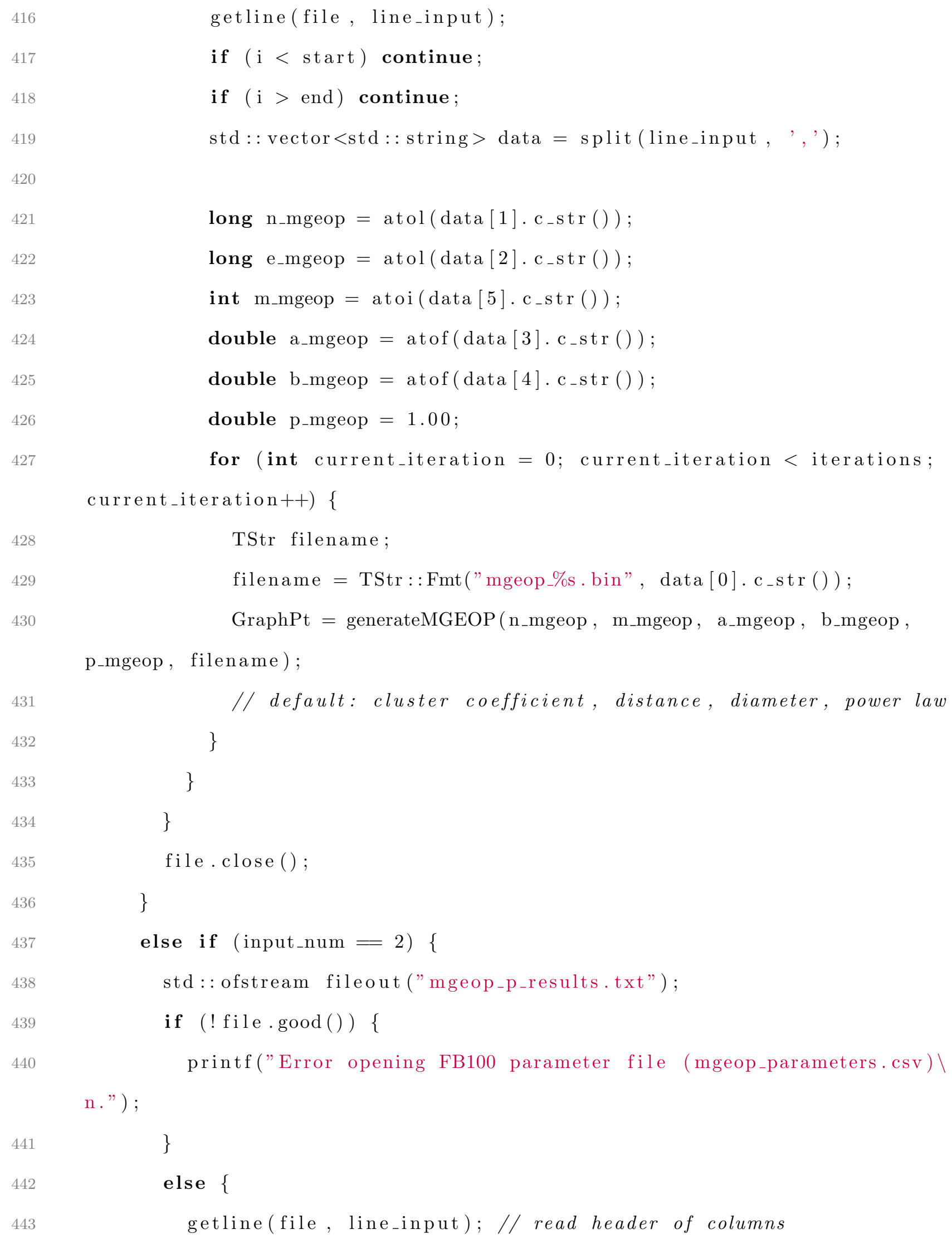


for (int $\mathrm{i}=0 ; \mathrm{i}<100 ; \mathrm{i}++)\{$

getline(file, line_input);

if $(\mathrm{i}<\mathrm{start})$ continue;

if $(\mathrm{i}>$ end ) continue;

std $:$ : vector $<$ std $::$ string $>$ data $=\operatorname{split}($ line_input , ', ' $)$;

TStr filename = TStr::Fmt("mgeop_\%s_p_0_compressed.smat", data

$\left.[0] \cdot c_{-} \operatorname{str}()\right)$;

GraphPt $=$ loadFile (filename);

TFlt e $=($ double $)$ GraphPt $->$ GetEdges ( ) ;

TFlt $\mathrm{v}=$ (double) GraphPt $->$ GetNodes ( ) ;

TInt maxDegree $=$ GetMaxDegNId (GraphPt);

int avgDegree $=($ int $)((2.0 *($ double $)$ GraphPt $\rightarrow$ GetEdges ()$/($ double

) GraphPt $\rightarrow$ GetNodes ()) + 0.5);

TInt $\operatorname{minDegree}=$ GetMnDegNId (GraphPt);

TFlt density $=(2 * \mathrm{e}) /(\mathrm{v} *(\mathrm{v}-1)) ;$

TStr output = TStr: : Fmt $(\% \mathrm{~s} \backslash \mathrm{t} \% \mathrm{~d} \backslash \mathrm{t} \% \mathrm{~d} \backslash \mathrm{t} \% \mathrm{~d} \backslash \mathrm{t} \% \mathrm{~d} \backslash \mathrm{t} \% \mathrm{~d} \backslash \mathrm{t} \% .3 \mathrm{f} \backslash \mathrm{n} "$, data

[0].c_str(), GraphPt $\rightarrow$ GetNodes(), GraphPt $\rightarrow$ GetEdges (), minDegree,

avgDegree, maxDegree, density);

fileout $<<$ output.CStr ();

\}

\}

fileout.close ();

\}

else if (input_num $=3$ ) \{

std:: ofstream fileout_ds_rai0 ("0_rai_mgeop_domintaing_results.txt");

std:: ofstream fileout_ds_rai1 ("1_rai_mgeop_domintaing_results.txt");

std:: ofstream fileout_ds_rai2 ("2_rai_mgeop_domintaing_results.txt");

std:: ofstream fileout_ds_rai3 ("3_rai_mgeop_domintaing_results.txt");

std:: ofstream fileout_ds_rai4 ("4_rai_mgeop_domintaing_results.txt"); 
std:: ofstream fileout_ds_rai5 ("5_rai_mgeop_domintaing_results.txt");

if $(! \mathrm{file} \cdot \operatorname{good}())\{$

printf("Error opening FB100 parameter file (mgeop_parameters.csv) n." );

\}

else \{

TStrV datasets;

getline(file, line_input); // read header of columns

for (int $\mathrm{i}=0 ; \mathrm{i}<100 ; \mathrm{i}++)\{$

getline (file, line_input);

if $(i<$ start $)$ continue;

if $(\mathrm{i}>$ end) continue;

std $:$ : vector $<$ std $::$ string $>$ data $=\operatorname{split}($ line_input, ', ') ;

TStr filename = TStr::Fmt("mgeop_\%s.bin", data[0].c_str()); datasets. Add (filename);

\}

for (int $\mathrm{z}=0 ; \mathrm{z}<\mathrm{datasets.Len}() ; \mathrm{z}++)\{$

TStr filename $=\operatorname{datasets}[\mathrm{z}]$;

GraphPt $=$ loadFile (filename);

for (int $\mathrm{k}=0 ; \mathrm{k}<=5 ; \mathrm{k}++) \quad\{$

TIntV del;

TIntPrV edges_del;

PUNGraph grp;

grp. New ();

grp $=$ TSnap : : GetKCore $($ GraphPt, k);

while $($ GetMnDegNId $(\operatorname{grp})<\mathrm{k})\{$ 
switch (k) \{

case 0 :

fileout_ds_rai $0<$ core.CStr () ;

break;

case 1:

fileout_ds_rai $1<$ core.CStr();

break;

case 2:

fileout_ds_rai $2<<$ core.CStr ();

break;

case 3 :

fileout_ds_rai3 $<$ core.CStr();

break;

case 4:

fileout_ds_rai4 $<$ core.CStr ();

break;

case 5:

fileout_ds_rai5 $<$ core.CStr();

break;

\}

\}

\}

fileout_ds_rai0.close (); fileout_ds_rai1.close (); fileout_ds_rai2.

close (); fileout_ds_rai3.close (); fileout_ds_rai4.close()；fileout_ds_rai5

. close ();

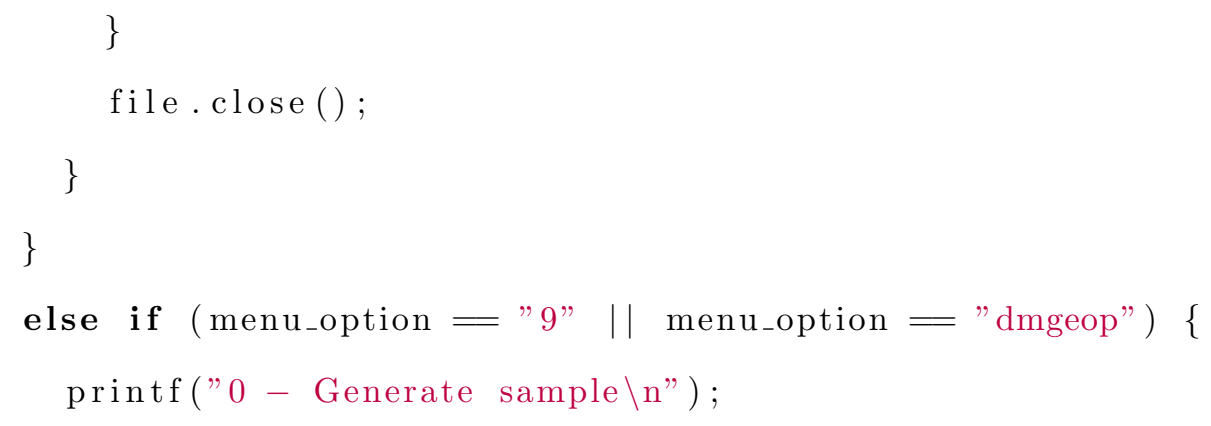


printf("1 - Generate from FB100 parameters $\backslash n ")$;

printf ("2 - General Chart $[+] \backslash \mathrm{n} ")$;

printf ("3 - Dominating Set (RAI) \n");

printf (" 4 - Power Law Graphs $\backslash n>")$;

std : : cin. getline (input, 256);

int input_num = atoi(input);

std:: string line_input;

if (input_num $=0)\{$

printf ("n =");

std: : cin.getline (input, 256);

int n_temp = atoi (input);

printf $(" \mathrm{~m}=")$;

std: : cin. getline (input, 256);

int m_temp = atoi (input);

printf ("a = ");

std : : cin.getline (input, 256);

double a_temp $=$ atof (input);

printf $(" b=")$;

std: : cin. getline (input, 256);

double b_temp = atof (input);

printf ("Iterations $=")$;

std: : cin. getline (input, 256);

int iterations = atoi(input);

TStr filename = TStr::Fmt("dmgeop_\%d_\%d. bin", n_temp, m_temp);

GraphPt $=$ generateMGEOPDistance (n_temp, m_temp, a_temp, b_temp ,

\section{filename );}

else if (input_num $=1)\{$ 


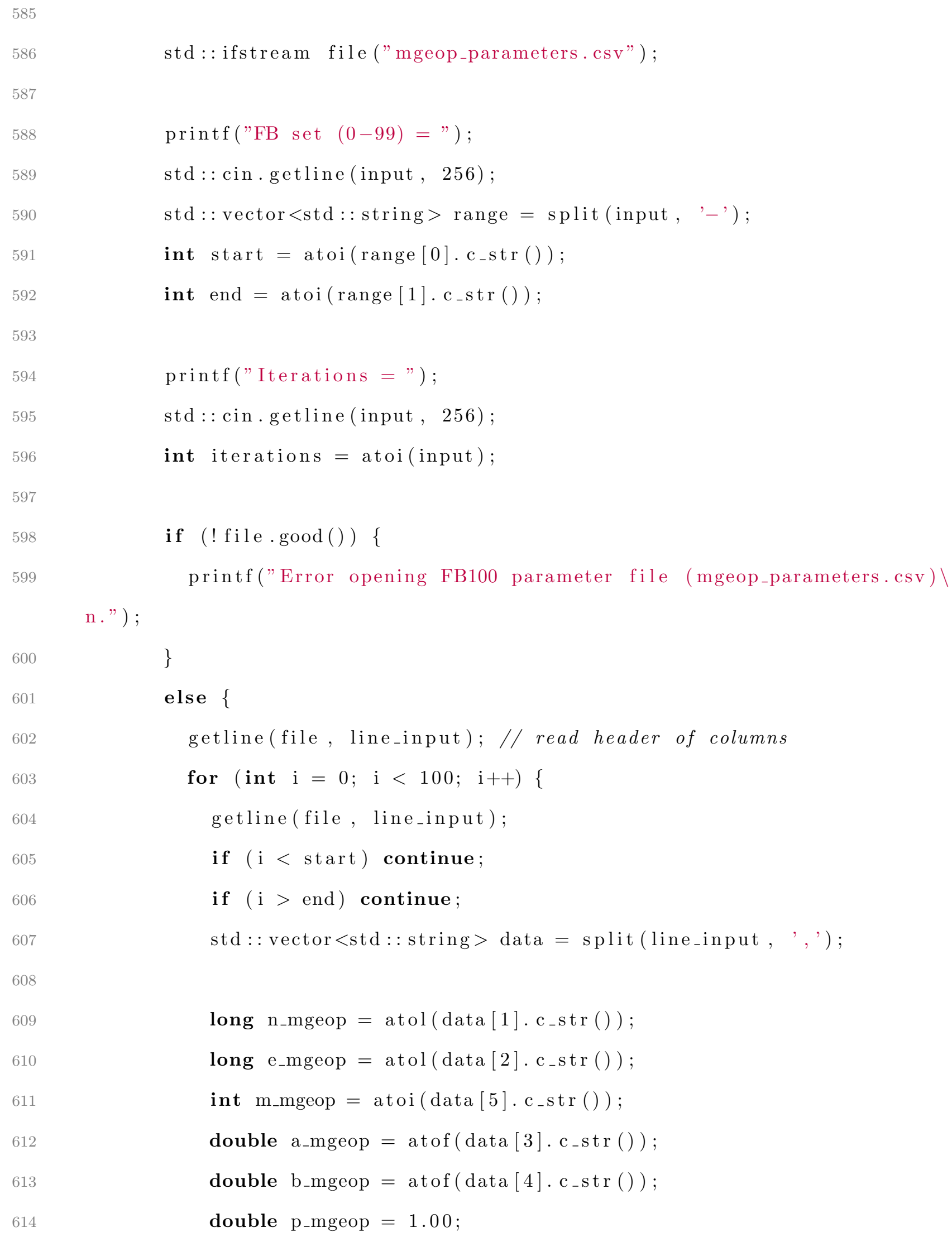

n." ) ;

printf ("FB set $(0-99)=")$;

std : : cin. getline (input, 256);

std $:$ : vector $<$ std $::$ string $>$ range $=$ split (input, '- ') ;

int $\operatorname{start}=\operatorname{atoi}\left(\operatorname{range}[0] \cdot \mathrm{c}_{-} \mathrm{str}()\right)$;

int end $=\operatorname{atoi}\left(\operatorname{range}[1] . c_{-} \operatorname{str}()\right)$;

printf ("Iterations $=")$;

std : : cin. getline (input, 256);

int iterations $=$ atoi(input);

if $(! \mathrm{file} \cdot \operatorname{good}())\{$

printf("Error opening FB100 parameter file (mgeop_parameters.csv)

\}

else \{

getline(file, line_input); // read header of columns

for (int i $=0 ; \mathrm{i}<100 ; \mathrm{i}++)\{$

getline (file, line_input);

if $(\mathrm{i}<\mathrm{start})$ continue;

if $(\mathrm{i}>$ end $)$ continue;

std : : vector $<$ std $::$ string $>$ data $=$ split (line_input, ', ') ;

long $\mathrm{n}_{-}$mgeop $=\operatorname{atol}\left(\operatorname{data}[1] \cdot \mathrm{c}_{-} \mathrm{str}()\right)$;

long e_mgeop $=\operatorname{atol}\left(\operatorname{data}[2] . c_{-} s t r()\right)$;

int m_mgeop $=\operatorname{atoi}\left(\operatorname{data}[5] \cdot c_{-}\right.$str ()$)$;

double a_mgeop $=\operatorname{atof}\left(\operatorname{data}[3] \cdot \mathrm{c}_{-} \mathrm{str}()\right)$;

double b_mgeop $=$ atof $\left(\operatorname{data}[4] \cdot c_{-}\right.$str ()$)$;

double p_mgeop $=1.00 ;$ 


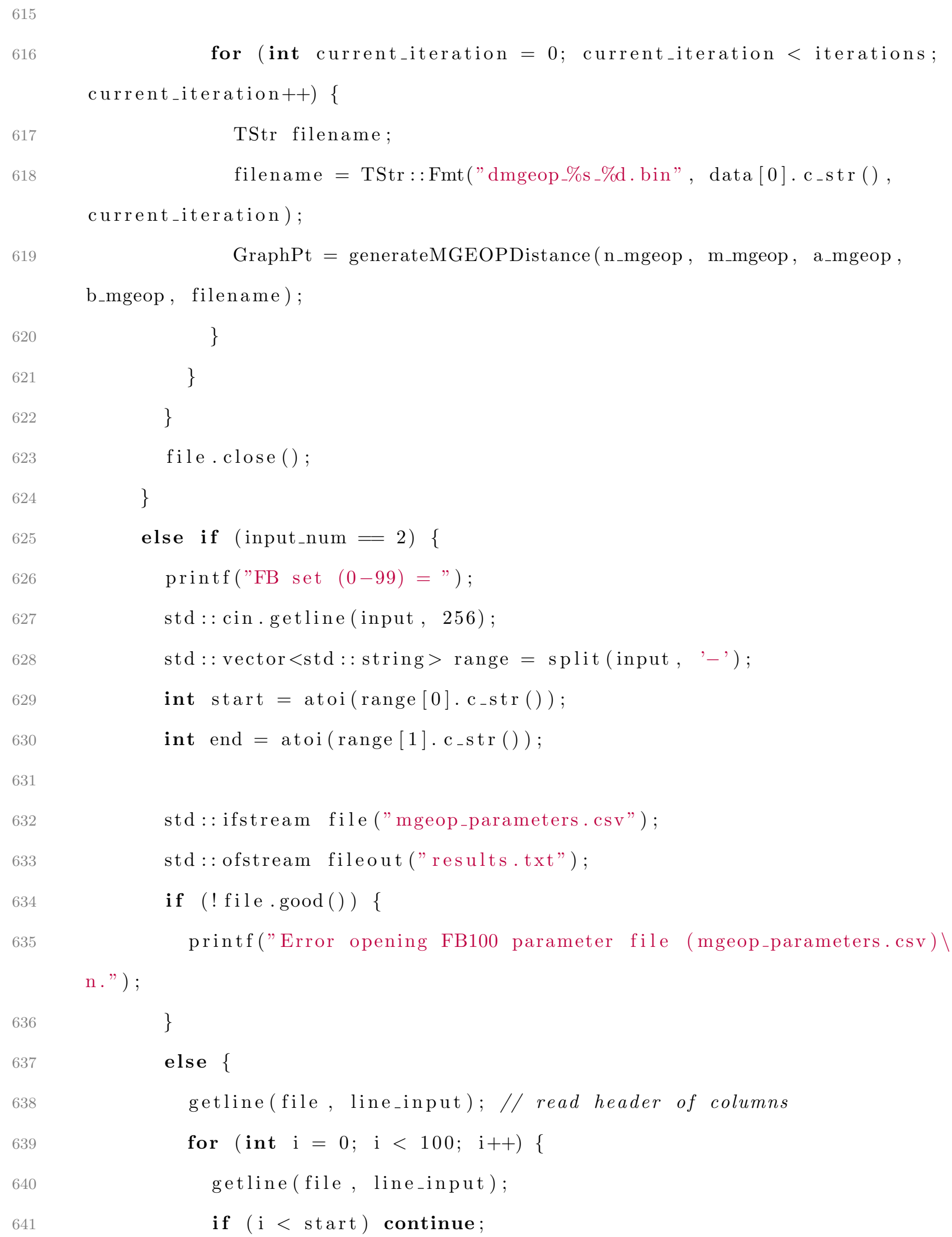

636

637

638

639

640

641

for (int current_iteration $=0$; current_iteration < iterations; current_iteration ++$)\{$

TStr filename;

filename = TStr: $:$ Fmt("dmgeop_\%s_\%d.bin", data $[0] . c_{-}$str () current_iteration);

GraphPt $=$ generateMGEOPDistance $\left(\mathrm{n} \_\right.$mgeop , m_mgeop , a_mgeop, b_mgeop, filename ); 
if $(\mathrm{i}>$ end $)$ continue;

std $:$ : vector $<$ std $::$ string $>$ data $=$ split $($ line_input , ', ') ;

TStr filename $=$ TStr::Fmt("dmgeop_\%s_0.bin", data [0]. c_str ());

GraphPt $=$ loadFile (filename);

TFlt $\mathrm{e}=($ double $)$ GraphPt $->$ GetEdges () ;

TFlt $\mathrm{v}=($ double $)$ GraphPt $->$ GetNodes ( ) ;

TInt $\operatorname{maxDegree}=$ GetMaxDegNId (GraphPt) ;

int avgDegree $=($ int $)((2.0 *($ double $)$ GraphPt $->$ GetEdges ()$/$ (double

) GraphPt $\rightarrow$ GetNodes ()) + 0.5);

TInt $\operatorname{minDegree}=\operatorname{GetMnDegNId}($ GraphPt $)$;

TFlt density $=(2 *$ e $) /(\mathrm{v} *(\mathrm{v}-1))$;

TStr output $=$ TStr : :Fmt $(" \% \mathrm{~s} \backslash \mathrm{t} \% \mathrm{~d} \backslash \mathrm{t} \% \mathrm{~d} \backslash \mathrm{t} \% \mathrm{~d} \backslash \mathrm{t} \% \mathrm{~d} \backslash \mathrm{t} \% \mathrm{~d} \backslash \mathrm{t} \% .3 \mathrm{f} \backslash \mathrm{n} "$, data [0].c_str (), GraphPt->GetNodes(), GraphPt->GetEdges(), minDegree, avgDegree, maxDegree, density);

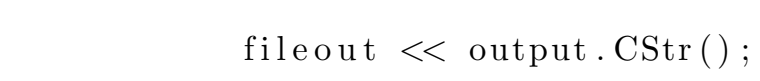


683
684
685
686
687
688
689
690
691
692

std:: ofstream fileout_ds_rai1("1_rai_dmgeop_domintaing_results.txt")

; std:: ofstream fileout_ds_rai2 ("2_rai_dmgeop_domintaing_results.txt") std : : ofstream fileout_ds_rai3 ("3_rai_dmgeop_domintaing_results.txt")

; std::ofstream fileout_ds_rai4 ("4_rai_dmgeop_domintaing_results.txt") ; ; ; std:: ofstream fileout_ds_rai5 ("5_rai_dmgeop_domintaing_results.txt")

printf $(" F B$ set $(0-99)=")$;

std: : cin.getline (input, 256);

std $:$ : vector $<\operatorname{std}::$ string $>$ range $=\operatorname{split}\left(\right.$ input,,${ }^{-}$');

int $\operatorname{start}=$ atoi $\left(\right.$ range $[0] \cdot c_{-}$str ()$)$;

int end $=$ atoi $\left(\operatorname{range}[1] \cdot c_{-}\right.$str ()$)$;

std:: ifstream file("mgeop_parameters.csv");

if $(! \mathrm{file} \cdot \operatorname{good}())\{$ printf("Error opening FB100 parameter file (mgeop_parameters.csv) n.");

\}

else \{

TStrV datasets;

getline(file, line_input); // read header of columns

for (int $\mathrm{i}=0 ; \mathrm{i}<100 ; \mathrm{i}++)\{$

getline (file, line_input);

if $(\mathrm{i}<\mathrm{start})$ continue;

if $(\mathrm{i}>$ end) continue;

std $:$ : vector $<\operatorname{std}:: \operatorname{string}>$ data $=\operatorname{split}($ line_input, ', ') ;

TStr filename $=$ TStr::Fmt("dmgeop_\%s_0.bin", data $[0] . c_{-}$str ()$)$; 


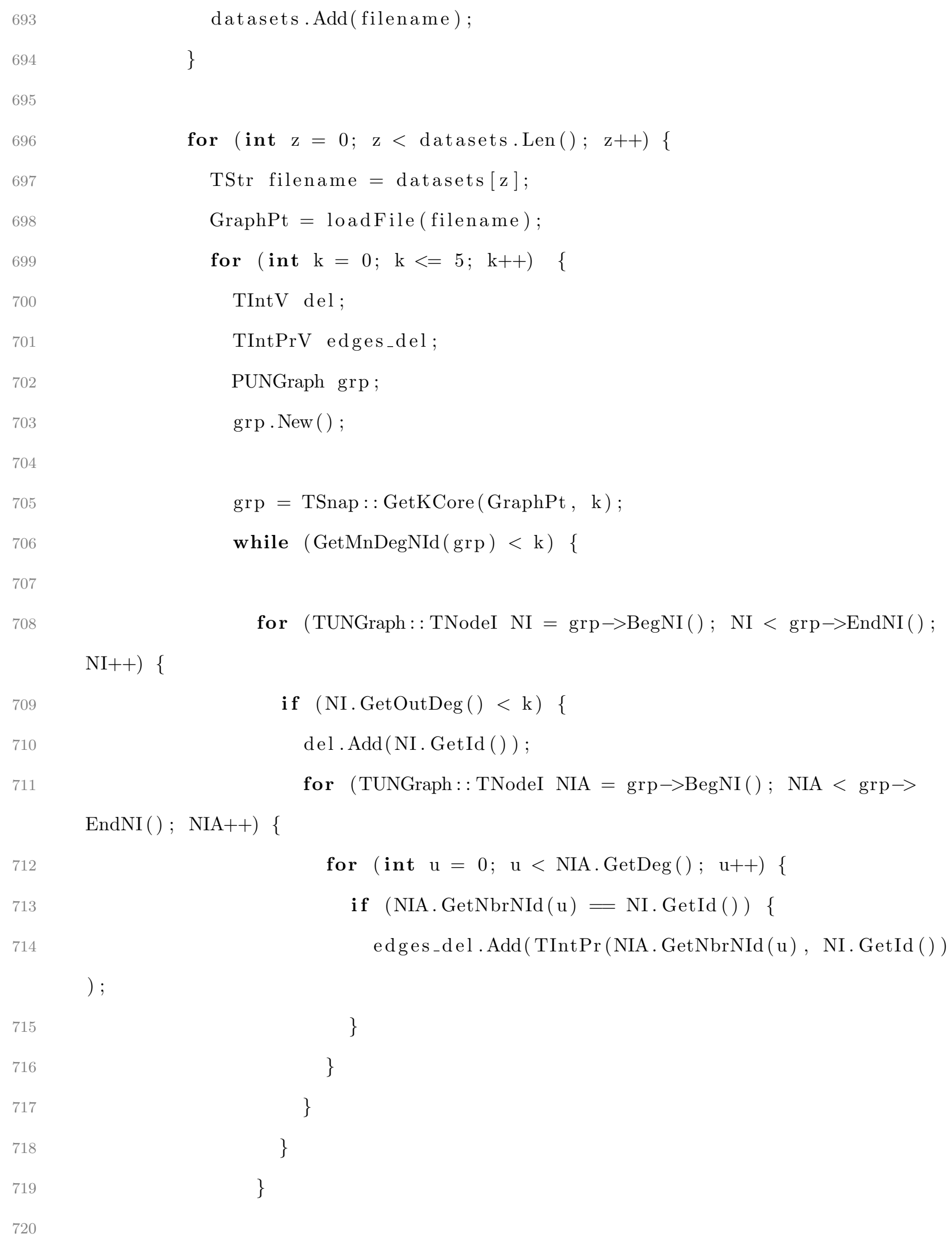

$$
\text { datasets. Add (filename); }
$$

\}

for (int $\mathrm{z}=0 ; \mathrm{z}<$ datasets. Len ()$; \mathrm{z}++)\{$

TStr filename $=$ datasets $[\mathrm{z}]$

GraphPt $=$ loadFile (filename $) ;$

for $($ int $\mathrm{k}=0 ; \mathrm{k}<=5 ; \mathrm{k}++) \quad\{$

TIntV del;

TIntPrV edges_del;

PUNGraph grp ;

$\operatorname{grp}$. New ()

$\operatorname{grp}=$ TSnap : : GetKCore (GraphPt, k ) ;

while $($ GetMnDegNId $(\operatorname{grp})<\mathrm{k})\{$

for (TUNGraph: : TNodeI NI $=\operatorname{grp} \rightarrow \operatorname{BegNI}() ; \mathrm{NI}<\operatorname{grp} \rightarrow$ EndNI () ; $\mathrm{NI}++)\{$ del . Add (NI. GetId ());

for $($ TUNGraph : : TNodeI NIA $=\operatorname{grp} \rightarrow$ BegNI ()$;$ NIA $<\operatorname{grp} \rightarrow$

$\operatorname{EndNI}() ; \mathrm{NIA}++)\{$

for $($ int $\mathrm{u}=0 ; \mathrm{u}<\mathrm{NIA} \cdot \operatorname{GetDeg}() ; \mathrm{u}++)\{$

if $(\operatorname{NIA} \cdot \operatorname{GetNbrNId}(\mathrm{u})=\operatorname{NI} \cdot \operatorname{GetId}())\{$ edges_del.Add (TIntPr(NIA.GetNbrNId $(u)$, NI.GetId ()$)$

) 
for (int del_len $=0 ;$ del_len $<$ del.Len ()$;$ del_len ++ ) \{ grp $\rightarrow$ DelNode (del [del_len ]);

\}

for (int del_len $=0 ;$ del_len $<$ del.Len ()$;$ del_len ++$)\{$ grp $\rightarrow$ DelEdge(edges_del [del_len]. Val1, edges_del [del_len ].

Val2);

\}

\}

$\operatorname{grp} \rightarrow$ Defrag () ;

GraphPt $=$ grp ;

printf("\%d,\%d $\backslash$ n", $\operatorname{grp} \rightarrow$ GetNodes (), $\operatorname{grp} \rightarrow$ GetEdges ()) ;

//TStr core;

if (input_num $=3)\{$

TIntV ds_rai_k = dominatingsetRAI (grp);

TStr core $=$ TStr $::$ Fmt $(" \% d \& \% d \backslash \backslash \backslash \backslash \backslash \backslash$ hline $\backslash$ n", grp $\rightarrow$ GetNodes ()

, ds_rai_k. Len ( ));

switch (k) \{

case 0 :

fileout_ds_rai $0<<$ core.CStr () ;

break ;

case 1 :

fileout_ds_rail $<<$ core.CStr () ;

break ;

case 2:

fileout_ds_rai $2<<$ core.CStr();

break ;

case 3 :

fileout_ds_rai $3<$ core. CStr();

break ; 


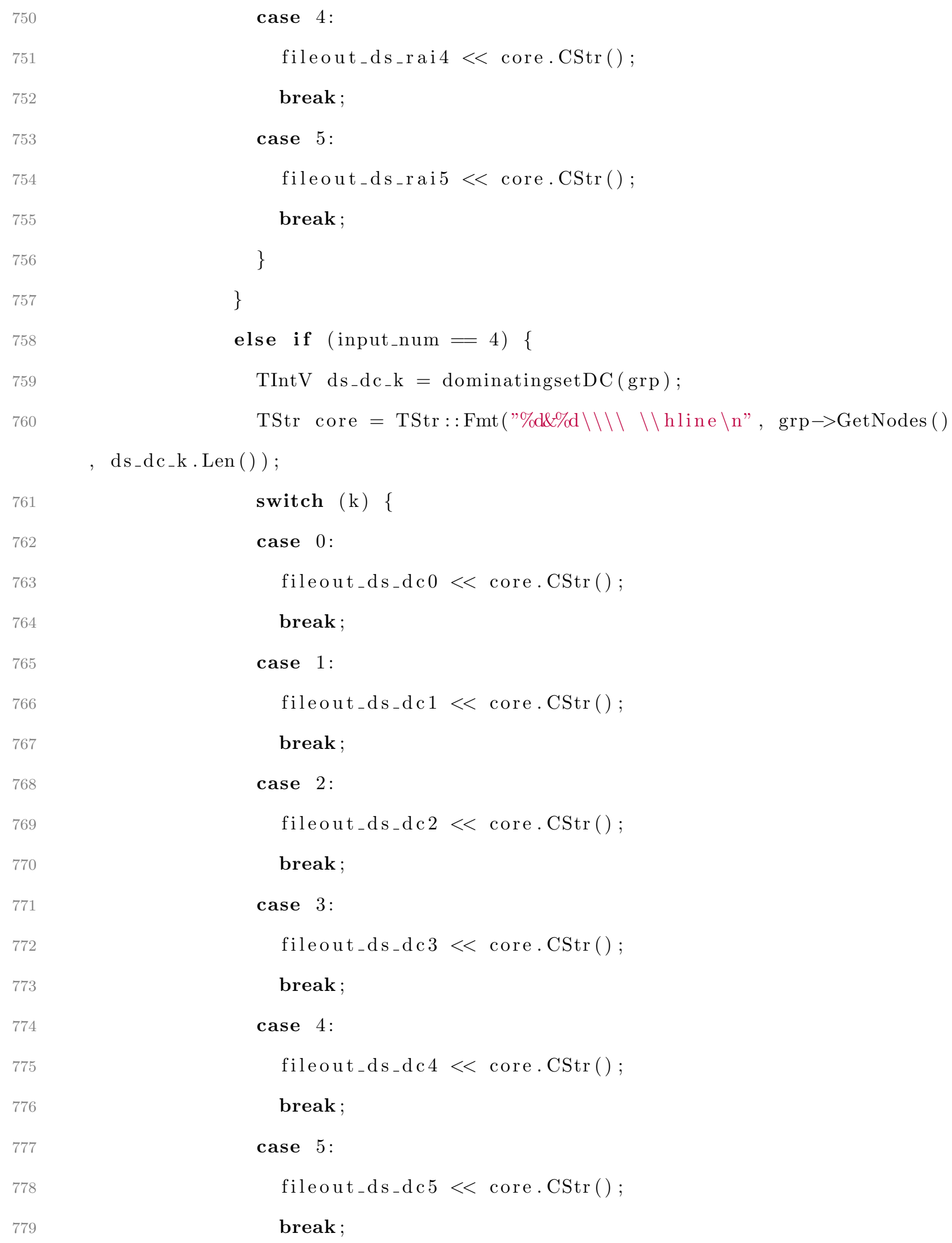

case 4:

fileout_ds_rai $4<<$ core. CStr () ;

break ;

case 5:

fileout_ds_rai5 $<<$ core. CStr () ;

break ;

\}

\}

else if (input_num $=4)\{$

TIntV ds_dc_k = dominatingsetDC ( grp);

TStr $\operatorname{core}=$ TStr $::$ Fmt $(" \% d \& \% d \backslash \backslash \backslash \backslash \backslash \backslash$ hline $\backslash n ", \operatorname{grp} \rightarrow$ GetNodes () , ds_dc_k. Len ( ) ) ;

\section{switch $(\mathrm{k})\{$}

case 0 :

fileout_ds_dc0 $0<\operatorname{core} \cdot \operatorname{CStr}()$;

break ;

case 1:

fileout_ds_dc1 $<$ core. CStr () ;

break ;

case 2:

fileout_ds_dc2 $2<$ core. CStr () ;

break ;

case 3 :

fileout_ds_dc $3<<$ core. CStr () ;

break ;

case 4:

fileout_ds_dc4 $4<$ core. $\operatorname{CStr}()$;

break ;

case 5 :

fileout_ds_dc $5<<$ core.CStr () ;

break ; 


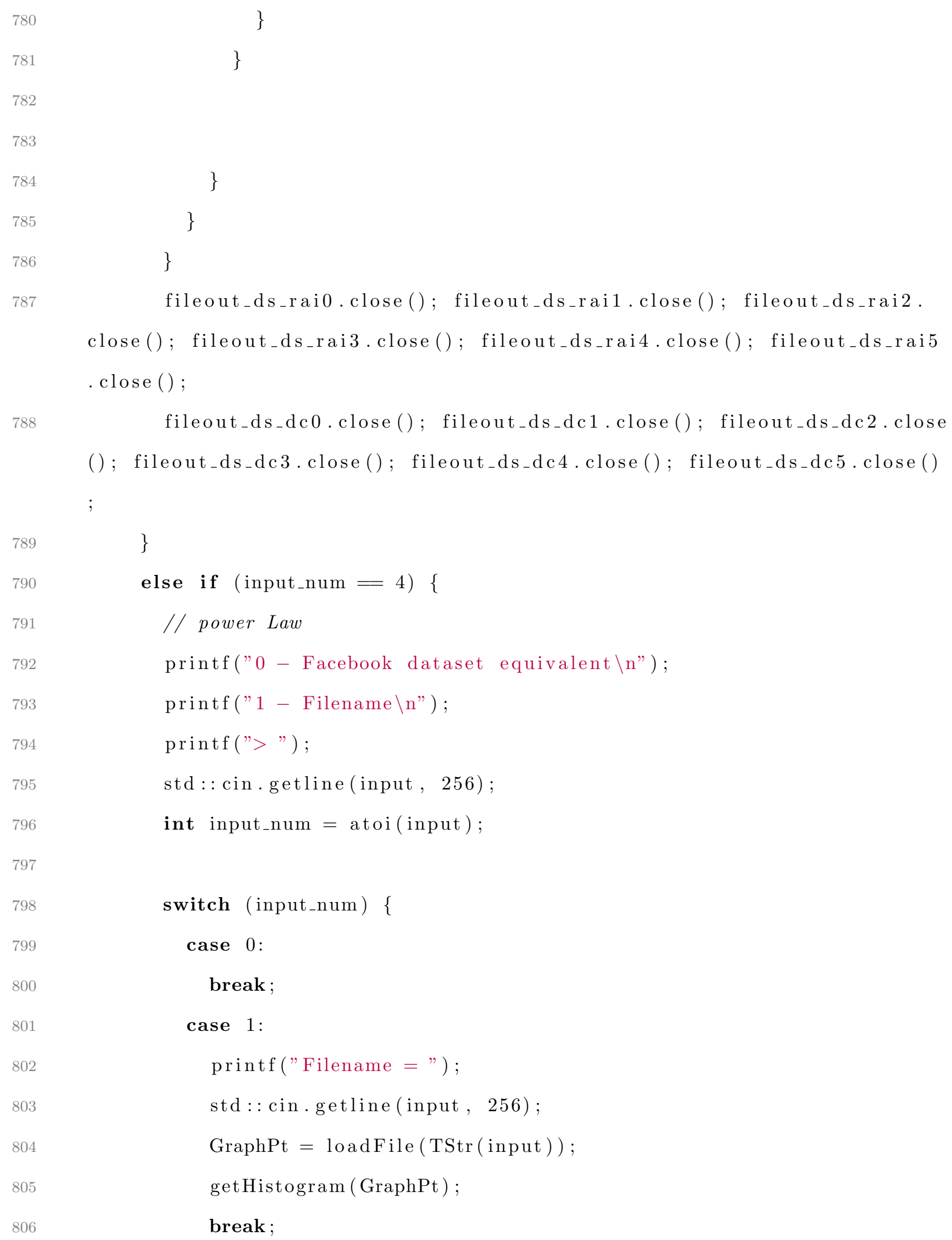

\}

fileout_ds_rai0.close (); fileout_ds_rail.close (); fileout_ds_rai2.

close (); fileout_ds_rai3.close ()$;$ fileout_ds_rai4_close ()$;$ fileout_ds_rai5 . close () ;

88

fileout_ds_dc0.close (); fileout_ds_dc1.close (); fileout_ds_dc2.close (); fileout_ds_dc3.close ()； fileout_ds_dc4.close ()； fileout_ds_dc5.close ( ) ; \} else if (input_num $=4)\{$ // power Law printf("0 - Facebook dataset equivalent $\backslash n ")$; printf ("1 - Filename $\backslash n ")$; printf $(">")$; std: : cin. getline (input, 256); int input_num = atoi (input);

switch (input_num) \{ case 0 : break ; case 1: printf ("Filename $=")$; std : : cin. getline (input, 256); GraphPt $=$ loadFile $($ TStr $($ input $))$; getHistogram (GraphPt) ; break ; 


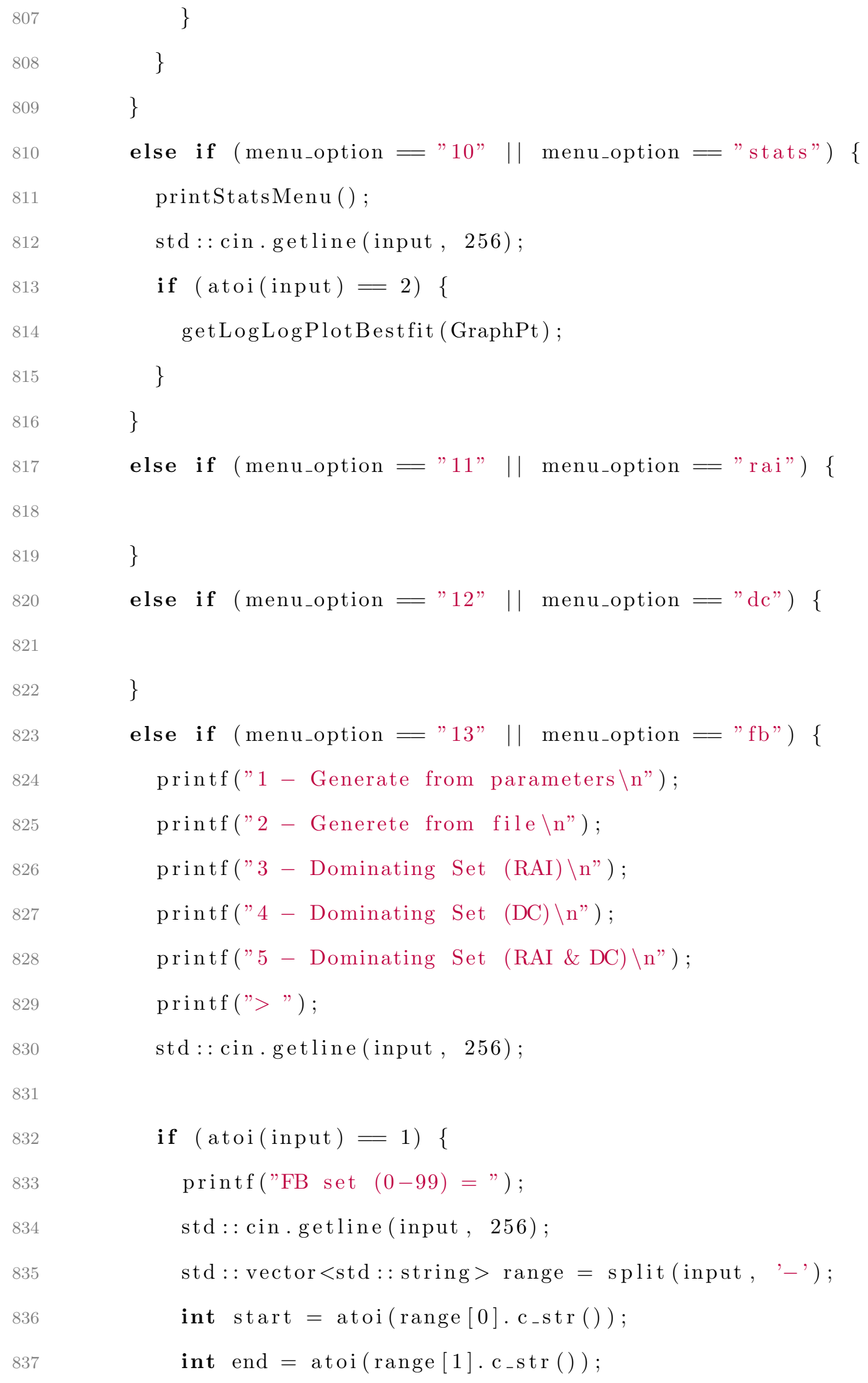




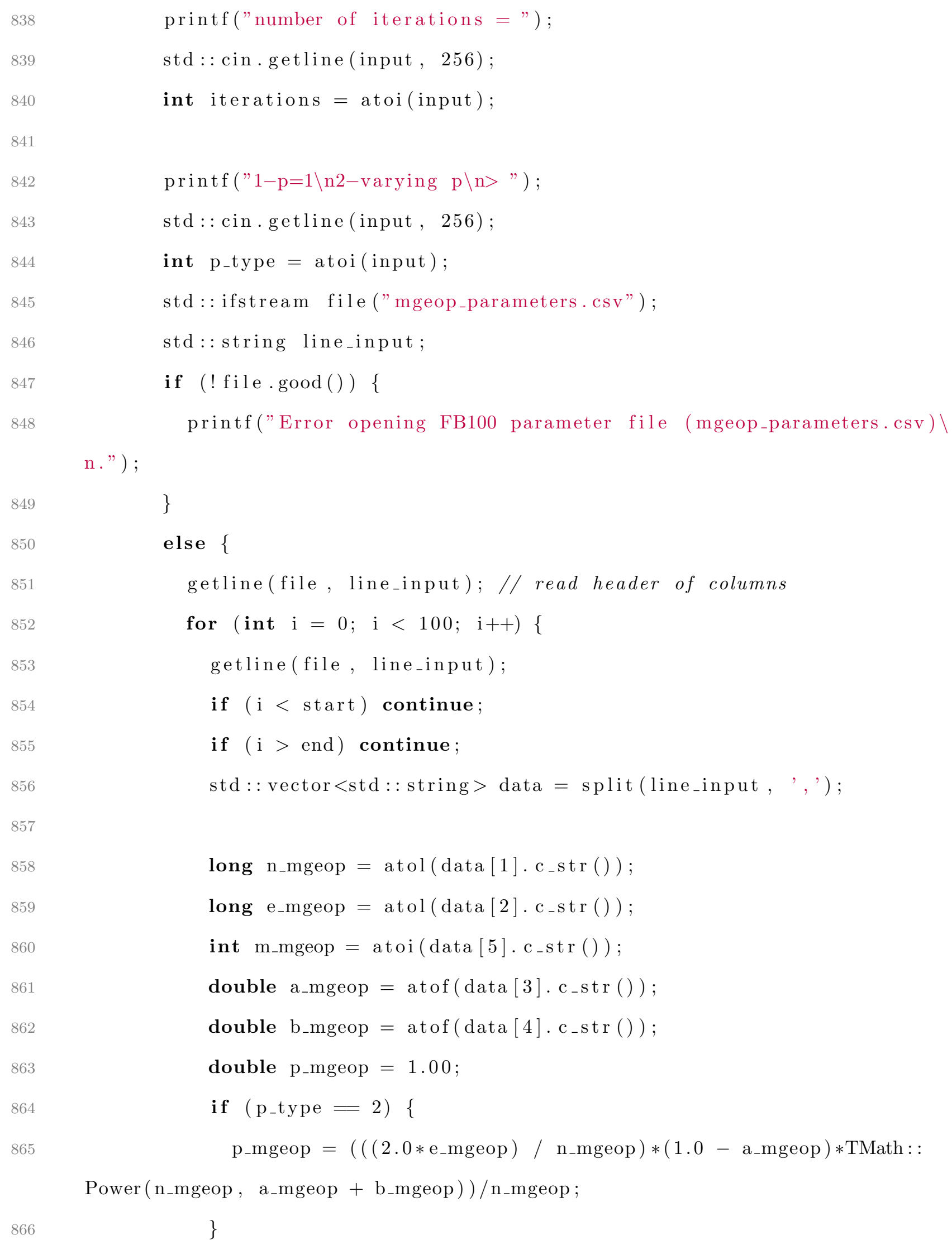

printf ("number of iterations =");

std : : cin. getline (input, 256);

int iterations = atoi(input);

printf $(" 1-\mathrm{p}=1 \backslash \mathrm{n} 2-\mathrm{varying} \quad \mathrm{p} \backslash \mathrm{n}>$ " $)$;

std : : cin. getline (input, 256);

int p_type = atoi (input);

std:: ifstream file ("mgeop_parameters.csv");

std:: string line_input;

if $(! \mathrm{file} \cdot \operatorname{good}())\{$

printf("Error opening FB100 parameter file (mgeop_parameters.csv) n."

\}

else \{

getline(file, line_input); // read header of columns

for (int i $=0 ; \mathrm{i}<100 ; \mathrm{i}++)\{$

getline (file, line_input);

if $(\mathrm{i}<\mathrm{start})$ continue;

if $(\mathrm{i}>$ end $)$ continue;

std $::$ vector $<$ std $::$ string $>$ data $=$ split (line_input, , ,');

long $\mathrm{n}_{-}$mgeop $=\operatorname{atol}\left(\operatorname{data}[1] \cdot \mathrm{c}_{-} \mathrm{str}()\right)$;

long e_mgeop $=\operatorname{atol}\left(\operatorname{data}[2] . c_{-}\right.$str ()$)$;

int m_mgeop $=\operatorname{atoi}\left(\operatorname{data}[5] \cdot \mathrm{c}_{-}\right.$str ()$)$;

double a_mgeop $=\operatorname{atof}\left(\operatorname{data}[3] \cdot c_{-}\right.$str ()$)$;

double b_mgeop $=\operatorname{atof}\left(\operatorname{data}[4] \cdot c_{-}\right.$str ()$)$;

double p_mgeop $=1.00$;

if $\left(\mathrm{p}_{-}\right.$type $\left.=2\right)\{$

p_mgeop $=(((2.0 *$ e_mgeop $) \quad /$ n_mgeop $) *(1.0-$ a_mgeop $) *$ TMath : :

Power (n_mgeop, a_mgeop + b_mgeop))/n_mgeop ; 
869

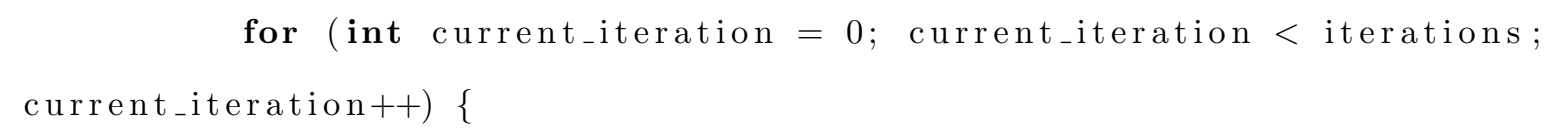

for (int current_iteration $=0 ;$ current_iteration $<$ iterations; current_iteration ++$)\{$

8




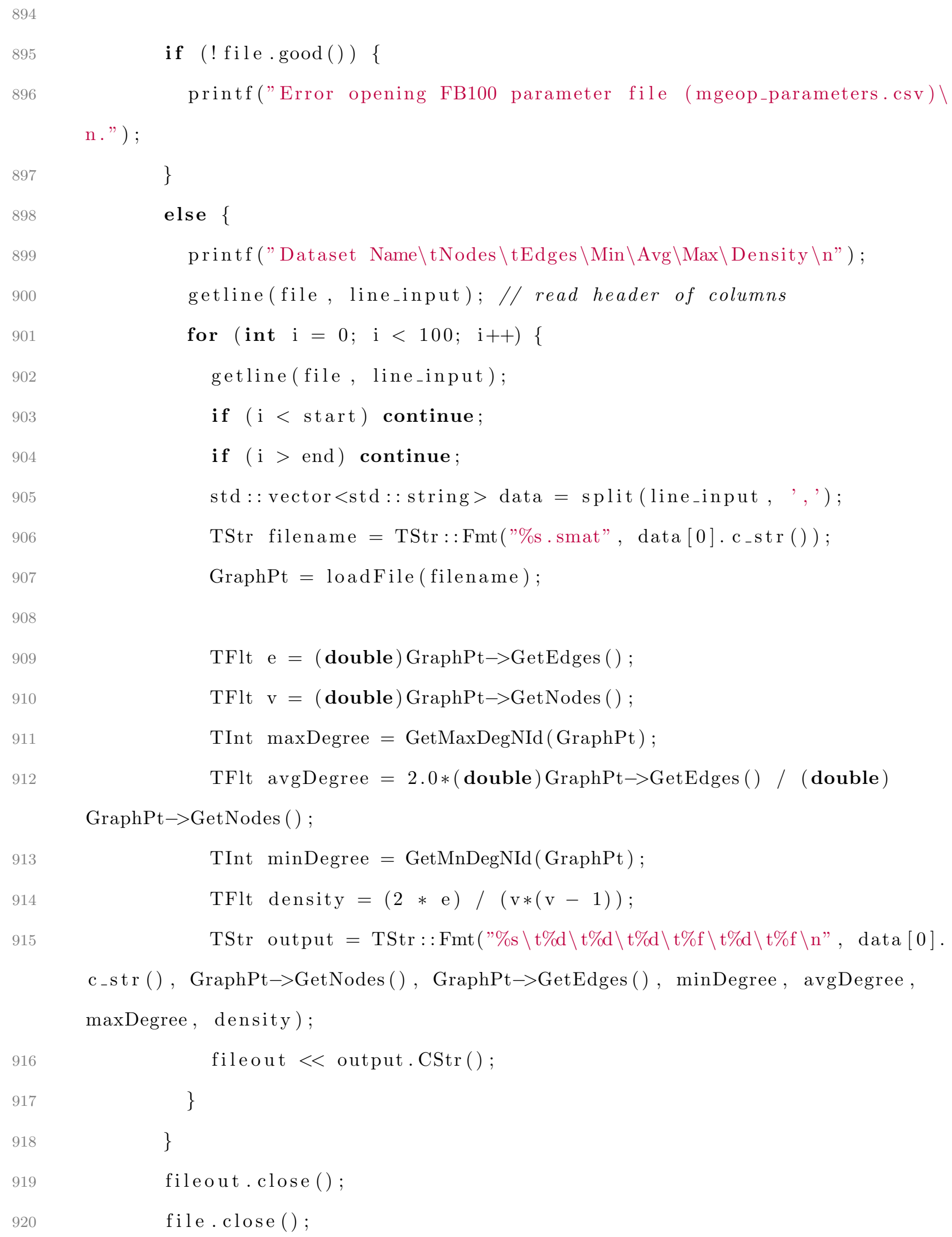

TFlt $\mathrm{e}=($ double $)$ GraphPt $->$ GetEdges ( ) ;

TFlt $\mathrm{v}=($ double $)$ GraphPt $->$ GetNodes ( ) ;

TInt maxDegree $=$ GetMaxDegNId (GraphPt);

TFlt avgDegree $=2.0 *($ double $)$ GraphPt $->\operatorname{GetEdges}() /($ double $)$

GraphPt $->$ GetNodes ( ) ; 


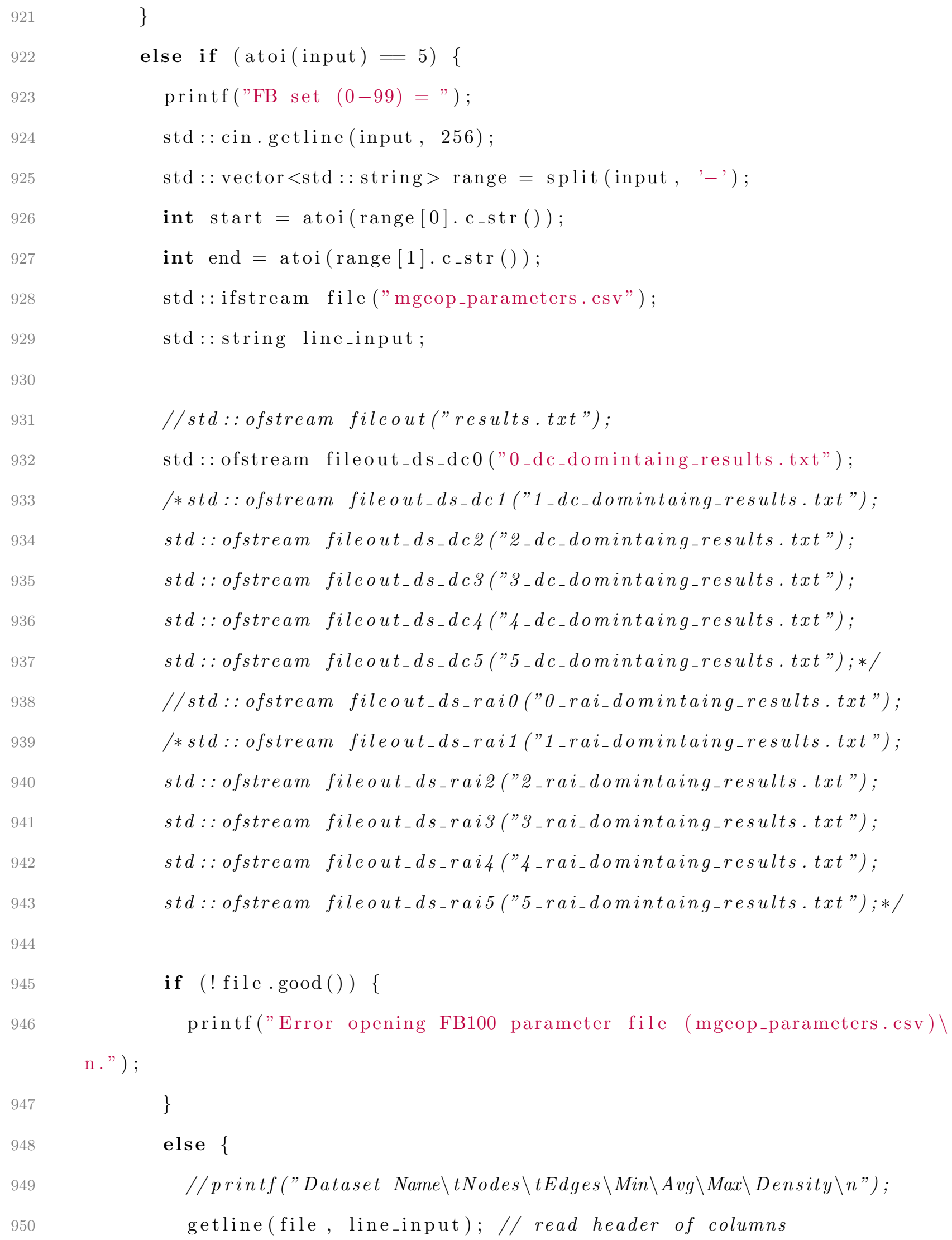
n." );

else if (atoi (input) $=5)\{$

printf ("FB set $(0-99)=")$;

std: : cin.getline(input, 256);

std $:$ : vector $<\operatorname{std}:: \operatorname{string}>$ range $=\operatorname{split}\left(\right.$ input,,$\left.-{ }^{\prime}\right)$;

int $\operatorname{start}=$ atoi $\left(\right.$ range $[0] \cdot c_{-}$str ()$)$;

int end $=$ atoi $\left(\operatorname{range}[1] \cdot \mathrm{c}_{-}\right.$str ()$)$;

std:: ifstream file("mgeop_parameters.csv");

std:: string line_input;

//std::ofstream fileout("results.txt");

std:: ofstream fileout_ds_dc0 ("0_dc_domintaing_results.txt");

/*std:: ofstream fileout_ds_dc1("1_dc_domintaing_results.txt");

std:: ofstream fileout_ds_dc2 ("2_dc_domintaing_results.txt");

std::ofstream fileout_ds_dc3("3_dc_domintaing-results.txt");

std:: ofstream fileout_ds_dc4 ("4_dc_domintaing_results.txt");

std::ofstream fileout_ds_dc5("5_dc_domintaing_results.txt");*/

//std::ofstream fileout_ds_raio("0_rai_domintaing_results.txt");

/*std::ofstream fileout_ds_rai1("1_rai_domintaing_results.txt");

std:: ofstream fileout_ds_rai2("2_rai_domintaing_results.txt");

std:: ofstream fileout_ds_rai3 ("3_rai_domintaing_results.txt");

std::ofstream fileout_ds_rai4("4_rai_domintaing_results.txt");

std::ofstream fileout_ds_rai5 ("5_rai_domintaing_results.txt");*/

if $(! \mathrm{file} \cdot \operatorname{good}())\{$

printf("Error opening FB100 parameter file (mgeop_parameters.csv)

\}

else \{

//printf("Dataset Name $\backslash$ tNodes $\backslash$ tEdges $\backslash$ Min $\backslash A v g \backslash M a x \backslash D$ ensity $\backslash n$ ");

getline(file, line_input); // read header of columns 


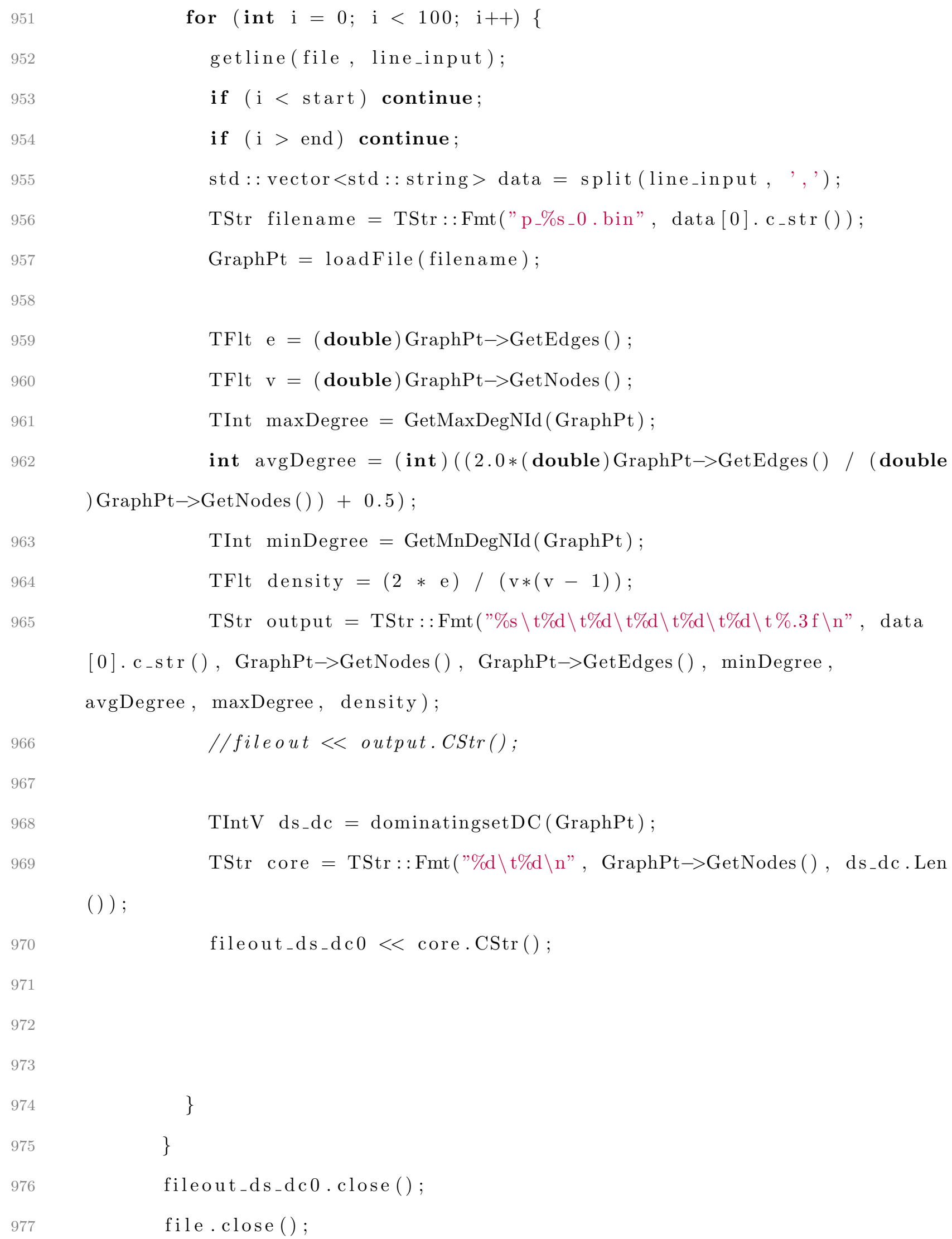

for (int $\mathrm{i}=0 ; \mathrm{i}<100 ; \mathrm{i}++)\{$

getline (file, line_input);

if $(\mathrm{i}<$ start $)$ continue;

if $(\mathrm{i}>$ end $)$ continue;

std $::$ vector $<$ std $:: \operatorname{string}>$ data $=$ split (line_input, ', ' ) ;

TStr filename $=$ TStr: $:$ Fmt (" p $_{-} \%_{s_{-}} 0$. bin", data $[0] \cdot c_{-}$str ()$)$;

GraphPt $=$ loadFile (filename);

TFlt $\mathrm{e}=$ (double) GraphPt $\rightarrow$ GetEdges ( ) ;

TFlt $\mathrm{v}=($ double $)$ GraphPt $->$ GetNodes ( ) ;

TInt $\operatorname{maxDegree}=\operatorname{GetMaxDegNId}($ GraphPt $)$;

int avgDegree $=($ int $)((2.0 *($ double $)$ GraphPt $\rightarrow$ GetEdges ()$/$ double

) GraphPt $\rightarrow$ GetNodes ( ) ) +0.5$)$;

TInt minDegree $=\operatorname{GetMnDegNId}($ GraphPt $)$;

TFlt density $=(2 * \mathrm{e}) /(\mathrm{v} *(\mathrm{v}-1))$;

TStr output = TStr: : Fmt $(" \% \mathrm{~s} \backslash \mathrm{t} \% \mathrm{~d} \backslash \mathrm{t} \% \mathrm{~d} \backslash \mathrm{t} \% \mathrm{~d} \backslash \mathrm{t} \% \mathrm{~d} \backslash \mathrm{t} \% \mathrm{~d} \backslash \mathrm{t} \% .3 \mathrm{f} \backslash \mathrm{n} "$, data

[0]. c_str (), GraphPt $\rightarrow$ GetNodes (), GraphPt $\rightarrow$ GetEdges (), minDegree,

avgDegree, maxDegree, density);

//fileout $<<$ output.CStr();

TIntV ds_dc $=$ dominatingsetDC (GraphPt);

TStr $\operatorname{core}=$ TStr $::$ Fmt $\left(" \% \mathrm{~d} \backslash \mathrm{t} \% \mathrm{~d} \backslash \mathrm{n} "\right.$, GraphPt $\rightarrow$ GetNodes ()$, \mathrm{ds} \_\mathrm{dc}$. Len

()) ; 


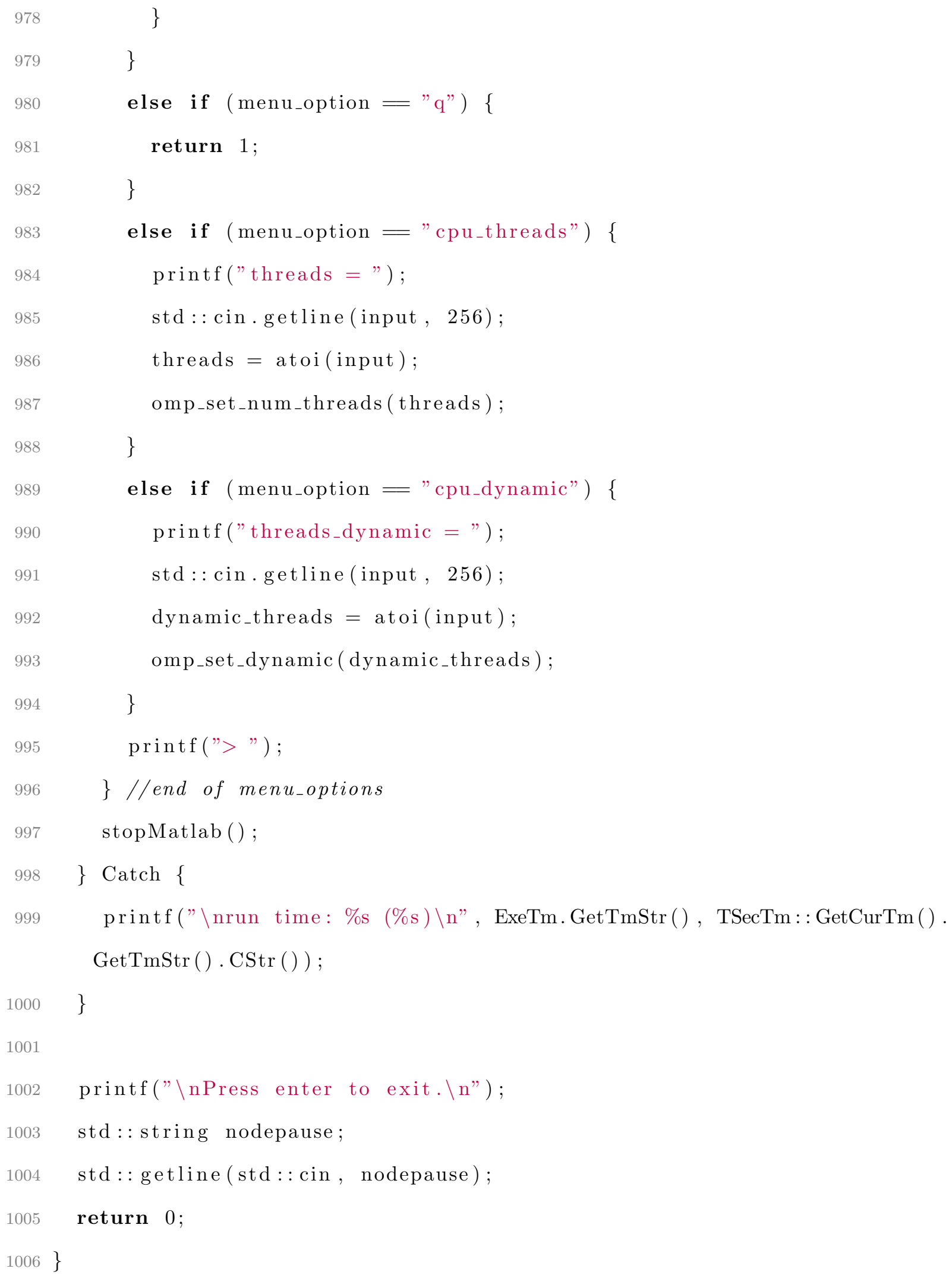




\section{loadFunctions.h}

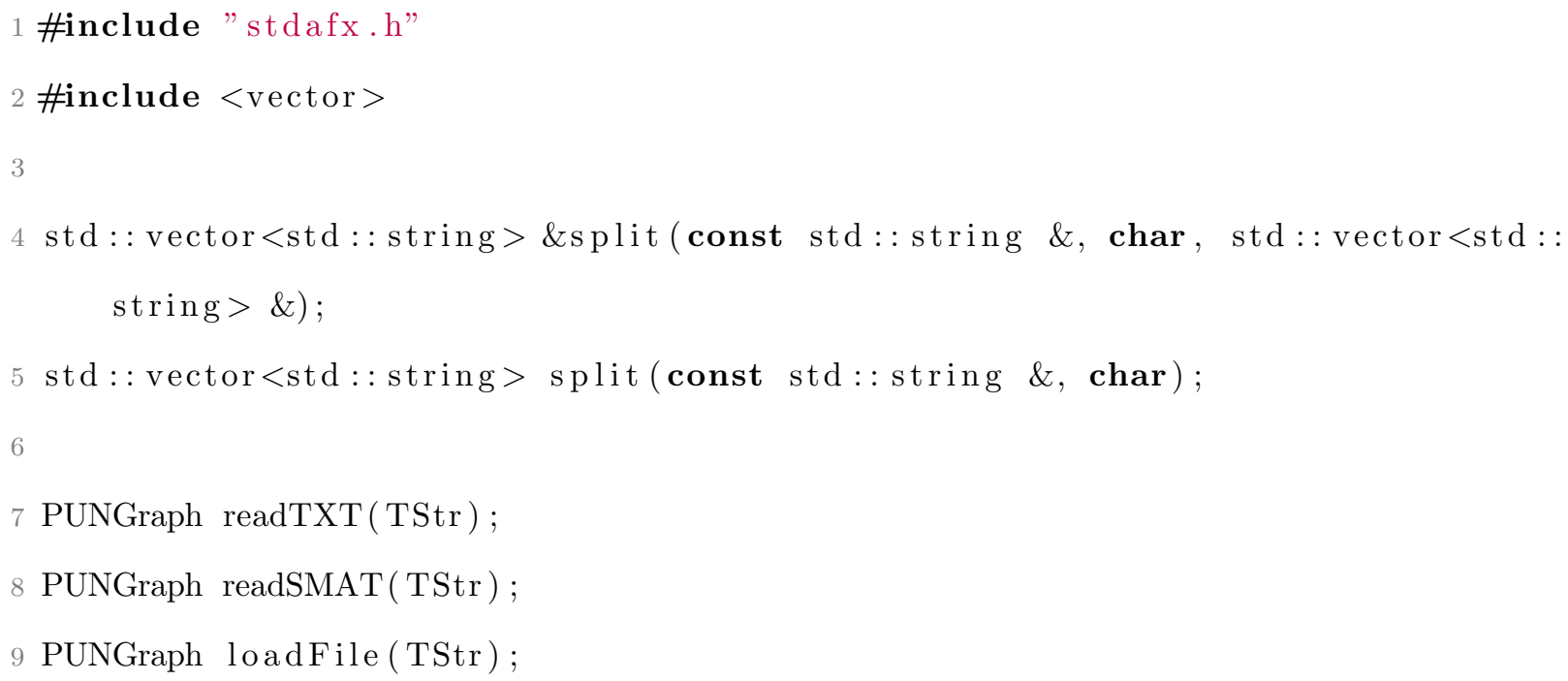


return elems;

$19\}$

20

21 PUNGraph readTXT(TStr filename) \{

22 PUNGraph GraphPt = TUNGraph: : New ();

23 int nodes1, nodes 2 ;

24 std::ifstream fin (filename.CStr());

25

$40\}$

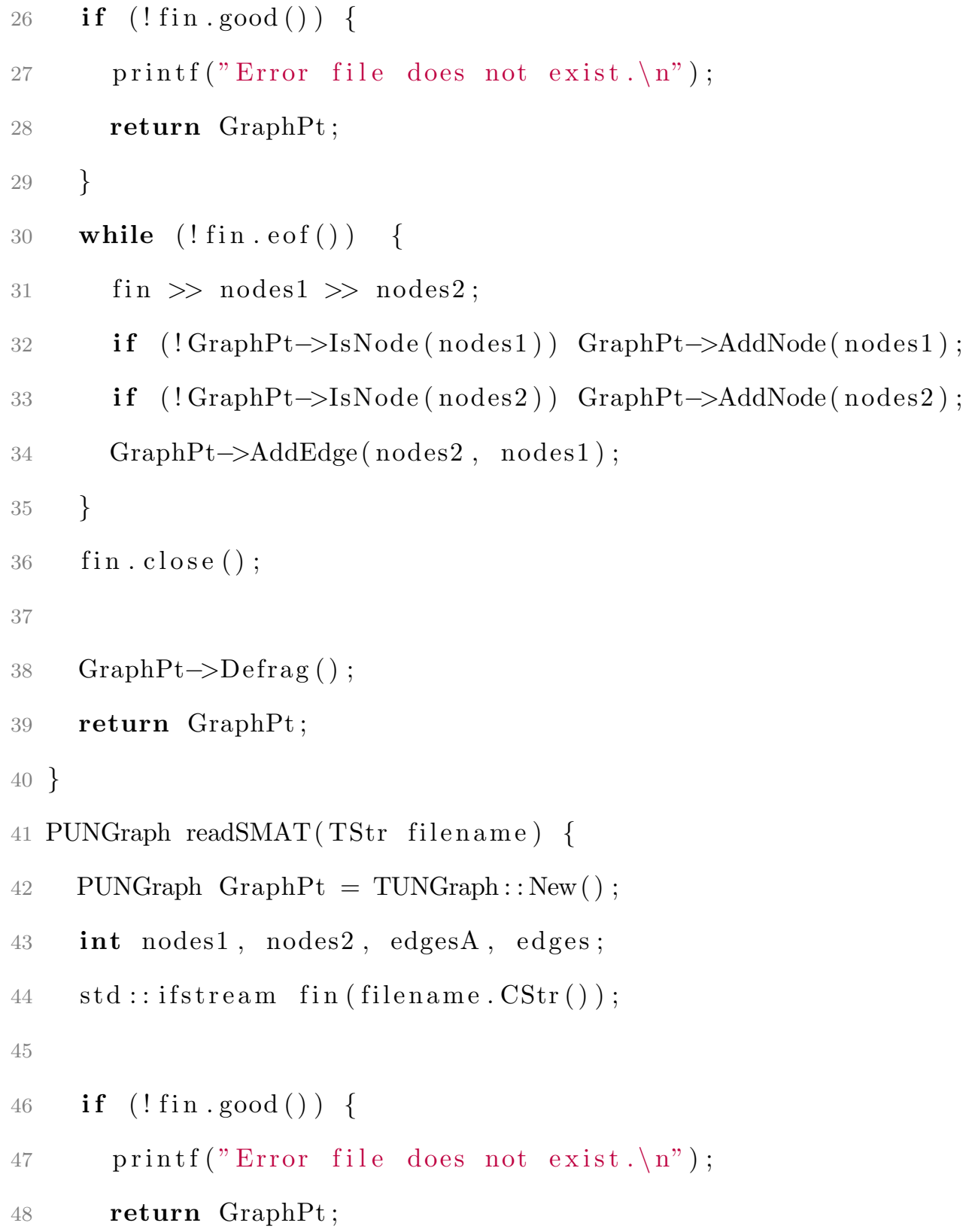

41 PUNGraph readSMAT(TStr filename) \{ 
$64\}$

65

66 PUNGraph loadFile(TStr file_name) \{

68

fin $>\operatorname{nodes} 1>\operatorname{nodes} 2>$ edges A;

for (int $\mathrm{i}=0 ; \mathrm{i}<\operatorname{nodes} 1 ; \mathrm{i}++)\{$

GraphPt->AddNode( i ) ;

\}

for $($ int $\mathrm{i}=0 ; \mathrm{i}<\operatorname{edgesA} ; \mathrm{i}++)\{$

fin $>$ nodes $1>\operatorname{nodes} 2>$ edges;

GraphPt->AddEdge(nodes2, nodes1);

\}

fin. close ();

GraphPt->Defrag ( ) ;

return GraphPt;

PUNGraph GraphPt $=$ TUNGraph : : New () ;

// load bin for faster loading $, \quad, ')$;

if (test_bin.good ()$)\{$

test_bin.close ();

TFIn FIn(file_name);

GraphPt $=$ TUNGraph $:: \operatorname{Load}($ FIn $) ;$

printf("Loading file: \%s...", file_name.CStr());

std $::$ vector $<$ std $::$ string $>$ names $=$ split (std : : string(file_name.CStr ()$)$. $c_{-}$str ()

std:: ifstream test_bin(names [0] + ". bin");

TStr file_name = TStr::Fmt("\%s.bin", names [0]. c_str()); 


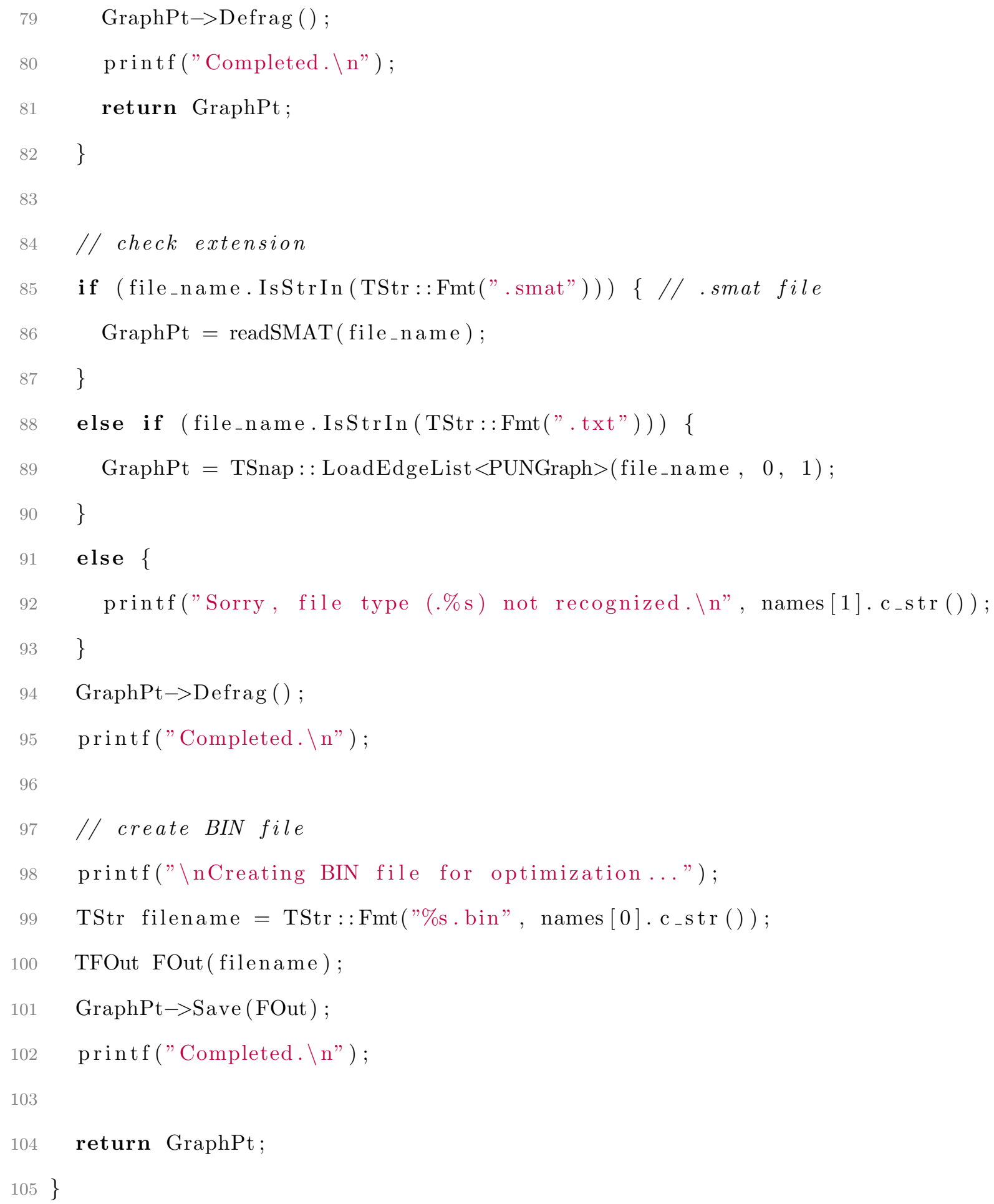


3 void TimerStart (LARGEINTEGER *);

4 double TimerQuery (LARGEINTEGER *);

5 PUNGraph generateGNP(const long, const double, TStr);

6 PUNGraph generateMGEOP (const long, const long, const double, const double, const double, TStr);

7 PUNGraph generateMGEOPDistance(const long, const long, const double, const double, TStr) ;

8 void GetHypercubeCoordinates (const int \&, TRnd \&, TFltV \&);

9 double getMaxElement (const TFltV \&, const TFltV \&, const TIntVV \&, int, int);

10 double GetInfinityDistance(const TFltV \&, const TFltV \&, const int, const TIntVV \&) ;

11 TIntVV generateCombinations (int, TIntV \&);

\section{graphFunctions.cpp}

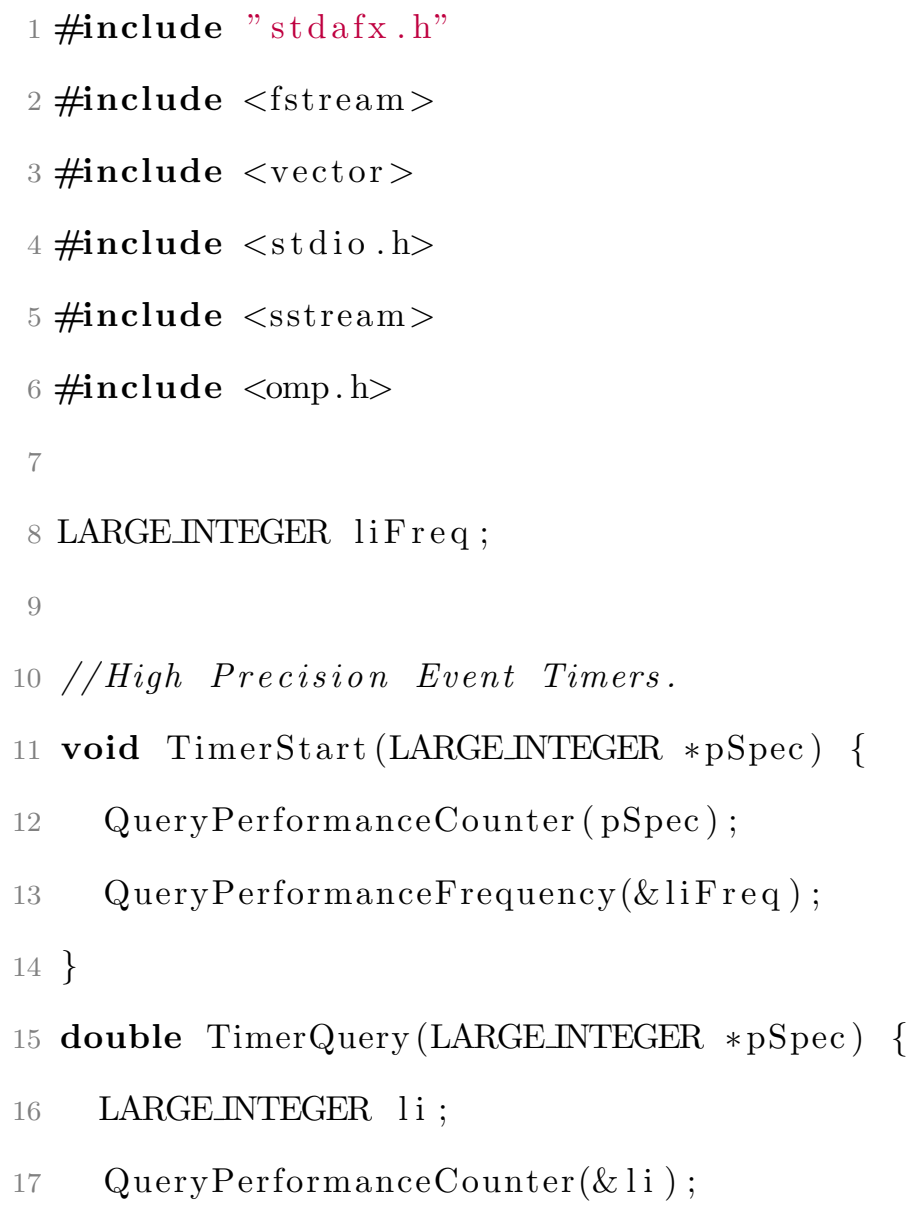


return ( li.QuadPart - pSpec->QuadPart) / (double)liFreq.QuadPart;

$19\}$

20

21 void GetHypercubeCoordinates (const int\& Dim, TRnd\& Rnd, TFltV\& ValV) \{

22 if (ValV.Len () != Dim) \{ValV.Gen(Dim); \}

23 for (int $\mathrm{i}=0 ; \mathrm{i}<\operatorname{Dim} ; \mathrm{i}++)\{$

$24 \quad$ ValV [i $]=$ Rnd.GetUniDev ( );

$25\}$

$26\}$

27

8 double getMaxElement (const TFltV\& $u$, const TFltV\& $v$, const TIntVV\& sets, int ii , int $\mathrm{m})\{$

29

30

double GetInfinityDistance (const TFltV\& u, const TFltV\& v, const int\& dim, const TIntVV\& sets ) \{

39 TFltV result;

$40 \quad$ int $\operatorname{size}=($ int $) \operatorname{pow}(3, \operatorname{dim}) ;$

41 result.Gen( size);

42 for (int $\mathrm{i}=0 ; \mathrm{i}<$ size; $\mathrm{i}++)\{$

$43 \operatorname{result}[\mathrm{i}]=\operatorname{getMaxElement}(\mathrm{u}, \mathrm{v}, \operatorname{sets}, \mathrm{i}, \operatorname{dim})$;

$44\}$

45 result.Sort (false);

46 return (double) result. Last (); 
48

49 TIntVV generateCombinations(int arraySize, TIntV\& possibleValues) \{

51 TIntV indices(arraySize);

52 int $\operatorname{comb}=($ int $) \operatorname{pow}(3$, arraySize $)$;

53 TIntVV sets (comb, arraySize);

54 int $\operatorname{count}=0$;

55 int $\mathrm{b}=0$;

56

for $($ int $\mathrm{q}=0 ; \mathrm{q}<$ arraySize $; \mathrm{q}++)\{$ indices $[\mathrm{q}]=0$;

$59\}$

60

do \{ 


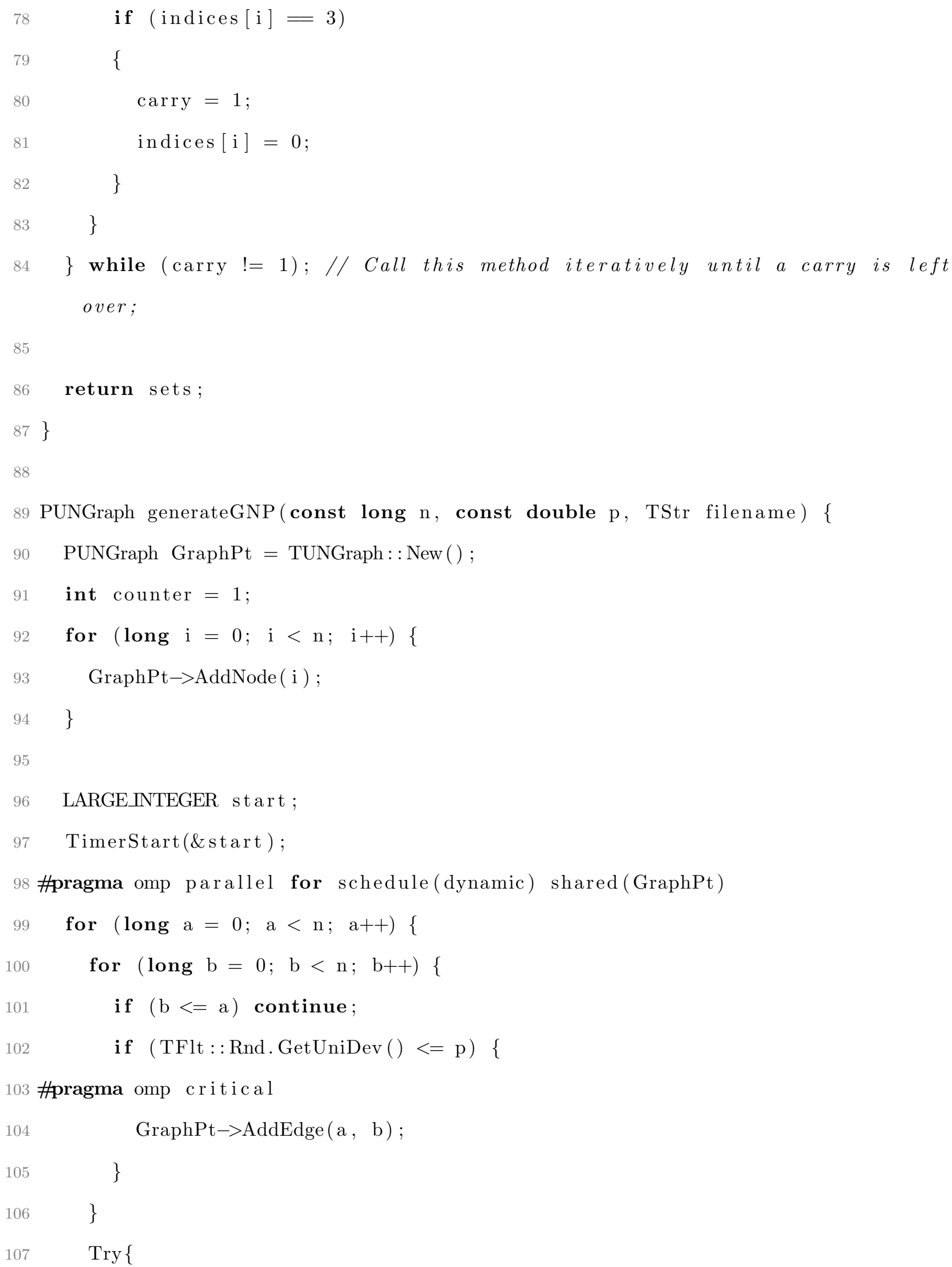


$122\}$

123

\}

double nPercentage $=($ double $)$ counter $/($ double $) \mathrm{n} ; / /$ Ranges from $0-1$

double nElapsed $=$ TimerQuery(\&start $) ; / /$ In seconds

double nTotalTime $=(1.0 /$ nPercentage $) *$ nElapsed;

double nRemaining $=$ nTotalTime - nElapsed; printf("\%2.1f\%\% complete, Estimated \%f seconds remaining \r", ( nPercentage $* 100.0)$, nRemaining);

counter $=$ counter +1 ;

\}

$\operatorname{Catch}\{\}$

// nothing

\}

GraphPt $\rightarrow$ Defrag ();

TFOut FOut (filename);

GraphPt $\rightarrow$ Save (FOut);

return GraphPt;

PUNGraph generateMGEOP(const long $\mathrm{n}$, const long $\mathrm{m}$, const double alpha, const double beta, const double p, TStr filename) \{

PUNGraph GraphPt $=$ TUNGraph : : New () ;

TFltV ValRadius;

TFltV ValV;

printf("Creating graph:\nAllocating nodes...\n");

ValRadius.Gen(n);

TVec $<$ TFltV $>$ coord ;

for (long $\mathrm{i}=0 ; \mathrm{i}<\mathrm{n} ; \mathrm{i}++)\{$

GetHypercubeCoordinates (m, TFlt:: Rnd, ValV);

/* For THESIS Sample

if $(i==0)\{$ 


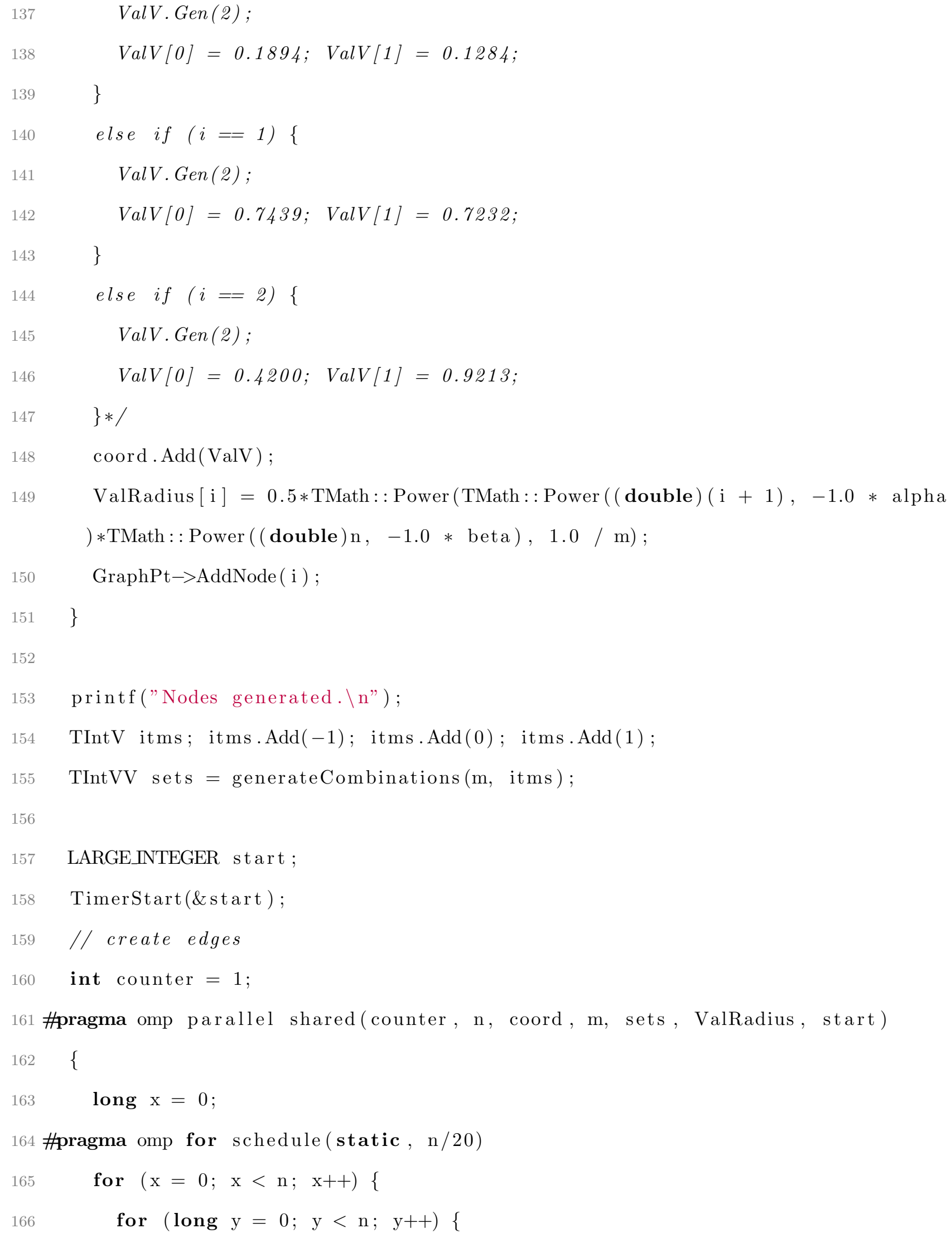

161 \#pragma omp parallel shared(counter, n, coord, m, sets, ValRadius, start)

$163 \quad$ long $\mathrm{x}=0$;

164 \#pragma omp for schedule (static, n/20)

165 for $(\mathrm{x}=0 ; \mathrm{x}<\mathrm{n} ; \mathrm{x}++)\{$

166

$$
\text { ValV.Gen(2); }
$$




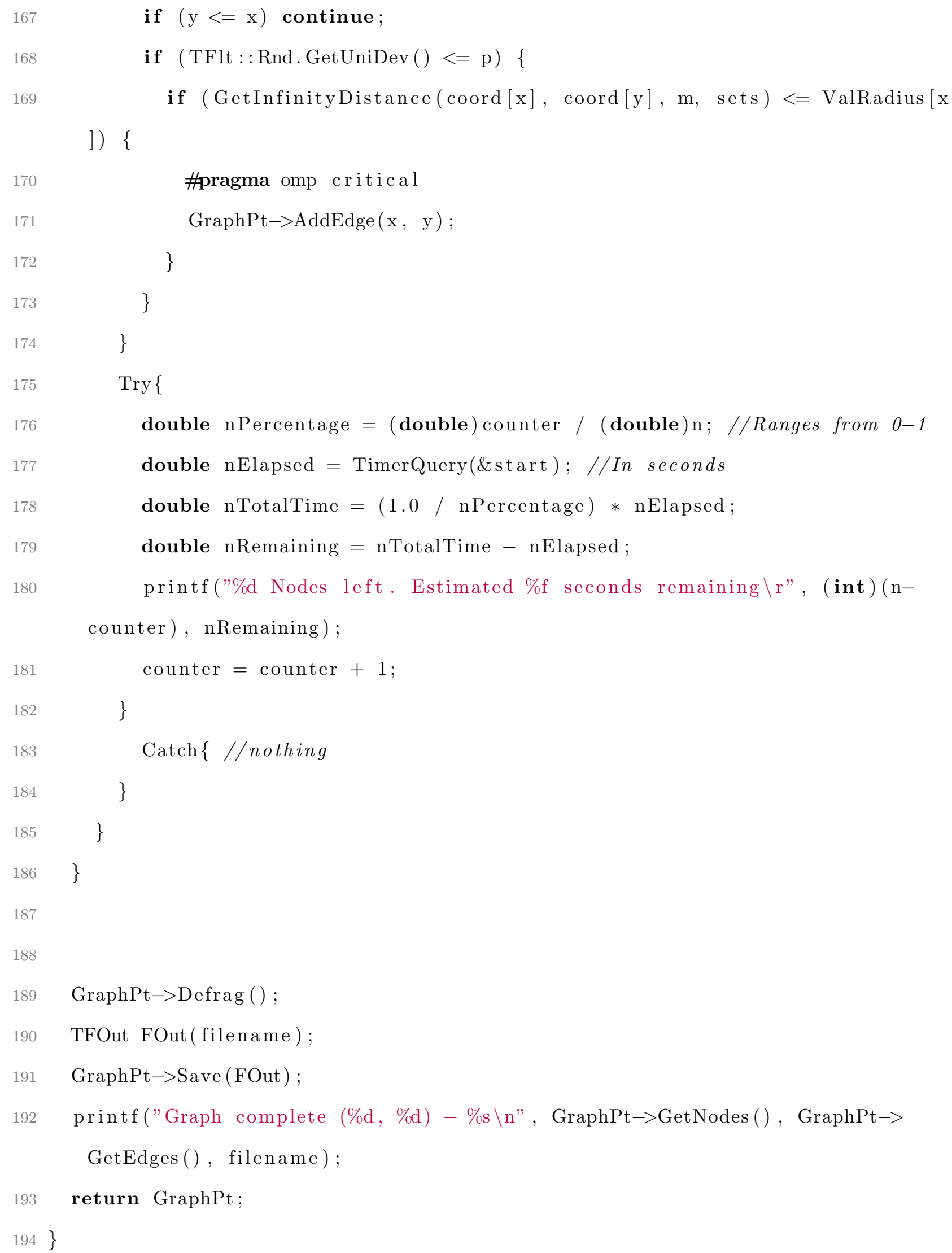

$194\}$ 
196 PUNGraph generateMGEOPDistance(const long $\mathrm{n}$, const long m, const double alpha, const double beta, TStr filename) \{

197

198

199

200

201

202

203

204

205

206

207

208

209

210

211

212

213

214

215

216

217

218

219

220

221

222 \#pragma omp parallel shared(counter, n, coord, m, sets, ValRadius, start)

printf("Creating graph:\nAllocating nodes...\n");

ValRadius.Gen(n);

// Generate random m-coordinates, radius for all nodes (rank is nodes id, since arbitrary)

for (long $\mathrm{i}=0 ; \mathrm{i}<\mathrm{n} ; \mathrm{i}++)\{$

GetHypercubeCoordinates (m, TFlt::Rnd, ValV);

coord. Add (ValV);

ValRadius $[\mathrm{i}]=0.5 *$ TMath: : Power (TMath: : Power $(($ double $)(\mathrm{i}+1),-1.0 *$ alpha )*TMath : : Power $(($ double $) \mathrm{n},-1.0 *$ beta $), 1.0 / \mathrm{m})$;

printf("Nodes generated.\n");

TIntV itms; itms.Add(-1); itms.Add(0); itms.Add(1);

TIntVV sets $=$ generateCombinations $(\mathrm{m}$, itms $) ;$

\section{LARGEINTEGER start;}

TimerStart(\&start);

// create edges

int counter $=1$; 


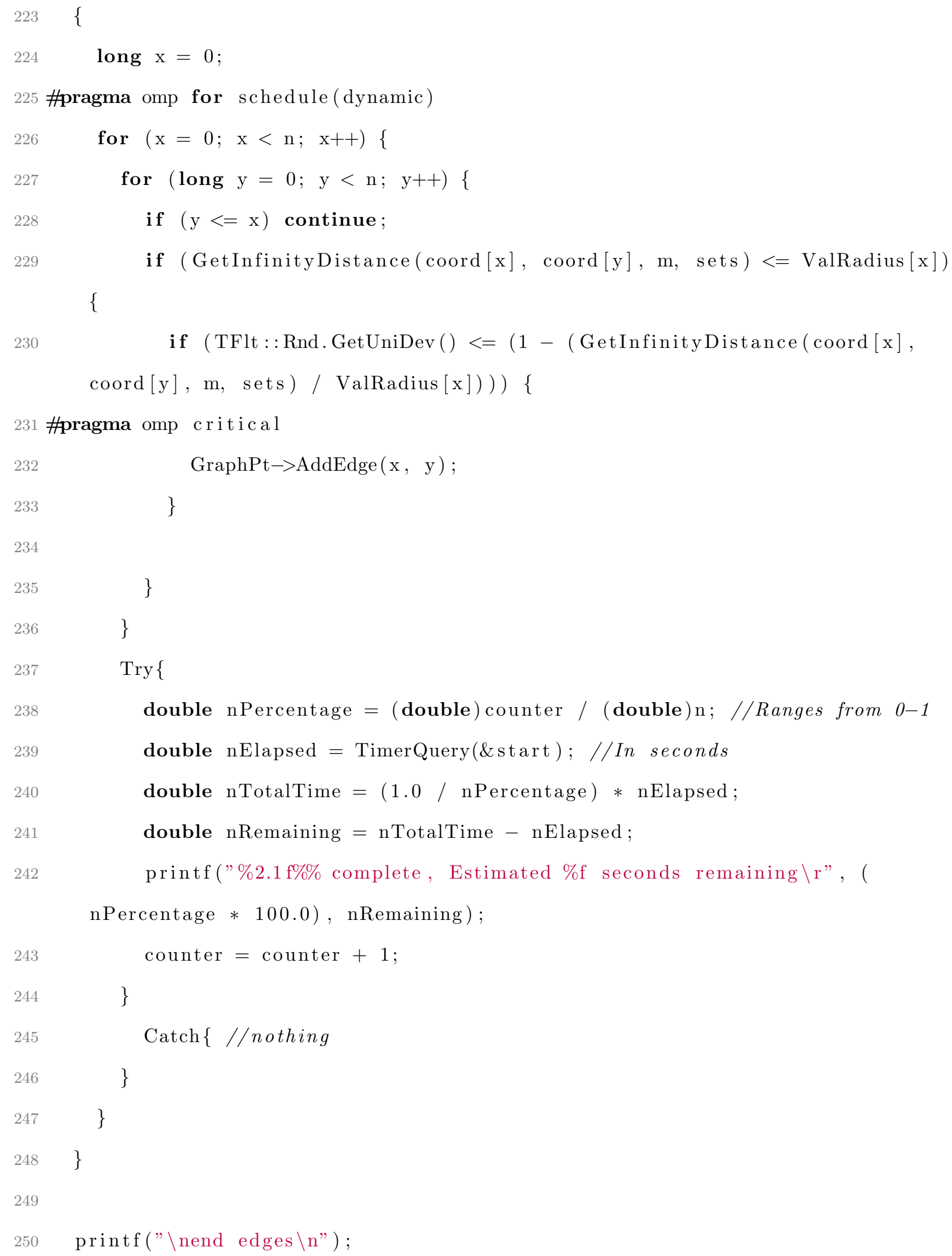


14 void startMatlab() \{

25 // Histogram

26 void getHistogram (const PUNGraph \&Graph) \{

27 std:: string evalaa("figure; histogram ([") ;

28

29

30

31

36 void printMatlabOutput() \{

37 if (buff $!=$ NULL)

$38 \quad$ printf(buff);

$39\}$

40

41 TFltPr bestPowerLawLocation (const PUNGraph \&Graph) \{

$42 / / x$ - degrees | $y$ - number of vertices 
TVec $<$ TIntPr $>$ degreeCount ;

for (PUNGraph:: TObj : : TNodeI NI $=$ Graph->BegNI ()$; \mathrm{NI}<\operatorname{Graph}->\operatorname{EndNI}() ; \mathrm{NI}++$ )

\{

$$
\text { bool exist }=\text { false }
$$$$
\text { for (int } \operatorname{tmp}=0 ; \operatorname{tmp}<\text { degreeCount.Len }() ; \operatorname{tmp}++)\{
$$$$
\text { if (degreeCount }[\mathrm{tmp}] \text {. Val1 }=\text { NI.GetDeg }())\{
$$ 
sum $+=$ TMath $:: \log (\mathrm{x}) *$ TMath $:: \log (\mathrm{y})$;

sum2 += TMath: : $\log (\mathrm{x})$;

sum3 += TMath : : $\log (\mathrm{y})$;

sum $4+=$ TMath $:: \log (\mathrm{x}) *$ TMath $:: \log (\mathrm{x})$;

\}

int $\mathrm{n} 2=\operatorname{int}(\mathrm{e})-\operatorname{int}(\mathrm{s}) ;$ if $(\mathrm{n} 2<0.1 * \mathrm{n})$ continue;

double $\mathrm{b}=((\mathrm{n} 2 * \operatorname{sum})-(\operatorname{sum} 2 * \operatorname{sum} 3)) /((\mathrm{n} 2 * \operatorname{sum} 4)-(\operatorname{sum} 2 * \operatorname{sum} 2))$;

if $($ b_max $>$ b) \{

$\mathrm{b} \_\max =\mathrm{b}$;

s_max $=\mathrm{s} ;$

\}

\}

for (double incre $=0$; incre $<0.5 ;$ incre $=$ incre +0.001$)\{$

double sum $=0.0 ;$

double $\operatorname{sum} 2=0.0 ;$

double $\operatorname{sum} 3=0.0 ;$

double $\operatorname{sum} 4=0.0 ;$

double $\mathrm{s}=\mathrm{s} \_$max

double $\mathrm{e}=(1-$ incre $) * \mathrm{n}$;

for $($ int $\operatorname{tmp}=\operatorname{int}(\mathrm{s}) ; \operatorname{tmp}<\operatorname{int}(\mathrm{e}) ; \operatorname{tmp}++)\{$

int $\mathrm{x}=$ degreeCount $[\mathrm{tmp}]$. Val1;

int $\mathrm{y}=$ degreeCount $[\mathrm{tmp}]$. Val2;

sum $+=$ TMath $:: \log (\mathrm{x}) *$ TMath $:: \log (\mathrm{y}) ;$

$\operatorname{sum} 2+=$ TMath : $\log (\mathrm{x})$;

sum3 += TMath : : $\log (\mathrm{y})$;

sum4 += TMath: : $\log (\mathrm{x}) *$ TMath $:: \log (\mathrm{x})$;

\}

int $\mathrm{n} 2=\operatorname{int}(\mathrm{e})-\operatorname{int}(\mathrm{s}) ;$ if $(\mathrm{n} 2<0.1 * \mathrm{n})$ continue;

double $\mathrm{b}=((\mathrm{n} 2 * \operatorname{sum})-(\operatorname{sum} 2 * \operatorname{sum} 3)) /((\mathrm{n} 2 * \operatorname{sum} 4)-(\operatorname{sum} 2 * \operatorname{sum} 2))$; 


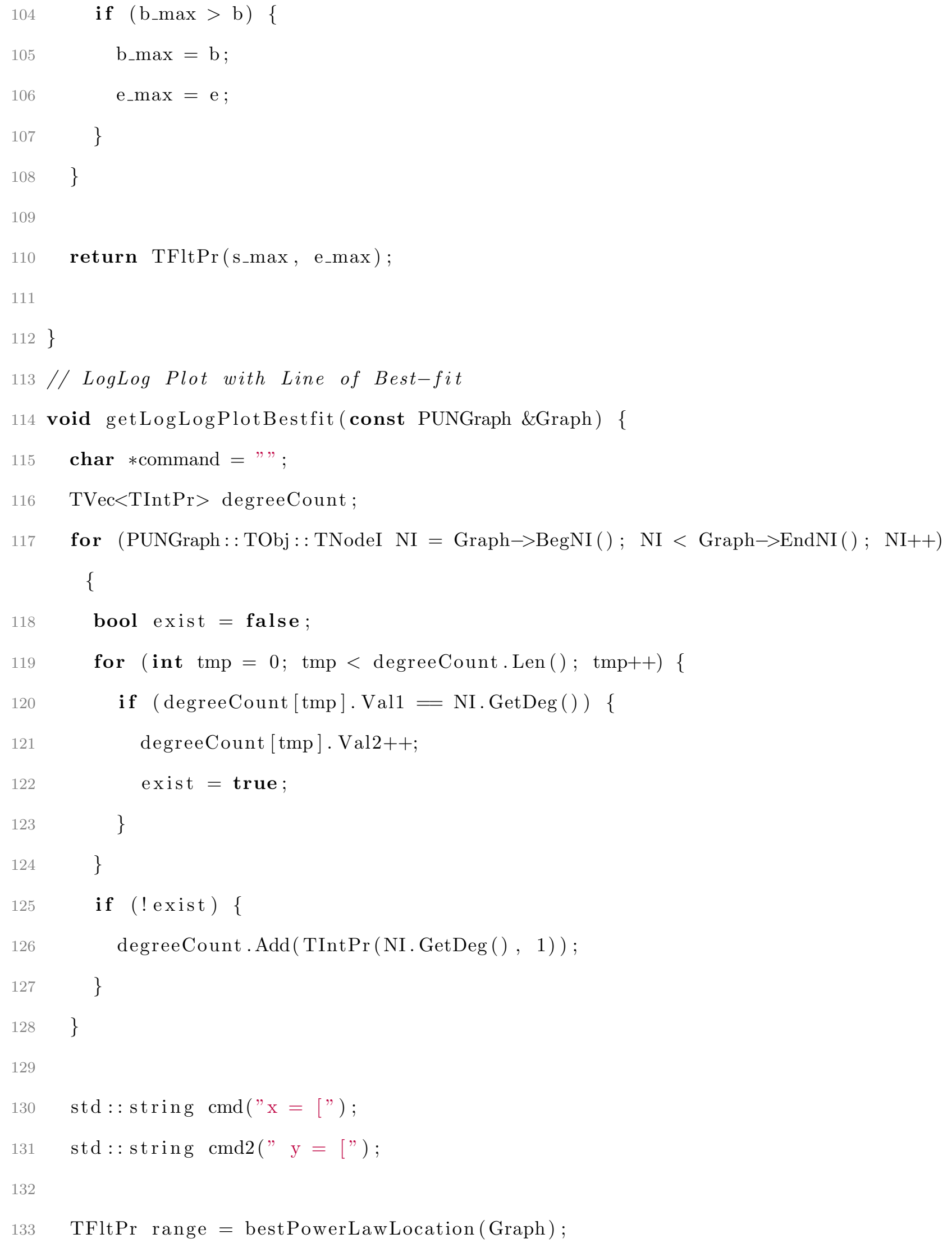


$154\}$

155

156

157 // LogLog Plot

158

\}

cmd $+="] ; "$;

$\operatorname{cmd} 2+="] ; "$;

cmd $+=\operatorname{cmd} 2$;

$$
\text { ;" ; }
$$

$$
\}
$$

\{

\}

for $($ int $\operatorname{tmp}=$ int $($ range. Val1 $) ; \operatorname{tmp}<$ int $($ range.Val2 $) ; \operatorname{tmp}++)\{$ cmd $+=$ std : : to_string (degreeCount $[$ tmp $]$. Val2 $)+"$; cmd2 += std : : to_string (degreeCount $[$ tmp $] \cdot$ Val1 $)+"$;

cmd $+="[\mathrm{x}$, index $]=\operatorname{sort}(\mathrm{x}) ; \mathrm{y}=\mathrm{y}(\operatorname{index}) ; \mathrm{y} 2=\mathrm{x} ; \mathrm{x}=\mathrm{y} ; \mathrm{y}=\mathrm{y} 2 ; " ;$

cmd $+="$ figure; hold on;";

cmd $+=" \log \log (\mathrm{x}, \mathrm{y}, \quad, \cdot) ; "$;

cmd $+=" p=$ polyfit $(\log (\mathrm{x}), \log (\mathrm{y}), 1) ; " ;$

cmd $+=" y_{-}$hat $=\exp (\mathrm{p}(1) * \log (\mathrm{x})+\mathrm{p}(2)) ; " ;$

cmd $+=" \log \log \left(\mathrm{x}, \mathrm{y}_{-}\right.$hat $) ; "$;

cmd $+="$ label $=[' \log (\mathrm{y})=, \operatorname{num} 2 \operatorname{str}(\mathrm{p}(1)) \quad \log (\mathrm{x})+\quad \operatorname{num} 2 \operatorname{str}(\mathrm{p}(2))] ; \quad$; cmd $+=$ "legend ('data', label); set (gca, 'xscale', 'log ') ; set (gca, ' yscale', ' log ')

engEvalString (ep, cmd. c_str ());

printMatlabOutput ();

void getLogLogPlot (const PUNGraph \&Graph) \{

std : : string evalaa ("figure; loglog ([");

for (PUNGraph: : TObj : :TNodeI NI = Graph $\rightarrow$ BegNI ()$;$ NI $<$ Graph $\rightarrow$ EndNI ()$;$ NI++)

$$
\text { evalaa }+=\operatorname{std}:: \text { to_string }(\text { NI.GetDeg }())+" " ;
$$


evalaa $+="]$ ) ; grid;";

164 engEvalString(ep, evalaa.c_str ());

$165\}$

166

167

168 // Stop MATLAB Engine

169 void stopMatlab () \{

170 eng Close (ep);

171 delete [] buff;

$172\}$ 


\section{Bibliography}

[1] L.A. Adamic, O. Buyukkokten, E. Adar, A social network caught in the web, First Monday 8 (2003).

[2] Y. Ahn, S. Han, H. Kwak, S. Moon, H. Jeong, Analysis of topological characteristics of huge on-line social networking services, In: Proceedings of the 16th International Conference on World Wide Web, 2007.

[3] N. Alon, J. Spencer, The Probabilistic Method, Wiley, New York, 2000.

[4] L. Backstorm, P. Boldi, M. Rosa, J. Ugander, S. Vigna, Four degrees of separation, In: Proceedings of the 4th Annual ACM Web Science Conference, 2012.

[5] A.L. Barabási, R. Albert, Emergence of scaling in random networks, Science 286 (1999) 509-512.

[6] P. M. Blau, Population structure and exchange process, Ethik Und Sozialwissenschaften 6 (1995) $20-22$.

[7] B. Bollobás, O. Riordan, J. Spencer, G. Tusnády, The degree sequence of a scale-free random graph process, Random Structures and Algorithms 18 (2001) 279-290.

[8] A. Bonato, A Course on the Web Graph, American Mathematical Society, Providence, Rhode Island, 2008.

[9] A. Bonato, D.F. Gleich, M. Kim, D. Mitsche, P. Pralat, A. Tian, S.J. Young, Dimensionality matching of social networks using motifs and eigenvalues, PLOS ONE 9 (2014) e106052.

[10] A. Bonato, N. Hadi, P. Horn, P. Prałat, C. Wang, Models of On-Line Social Networks, Internet Mathematics 6 (2011) 285-313.

[11] A. Bonato, J. Janssen, P. Prałat, Geometric protean graphs, Internet Mathematics 8 (2012) 2-28.

[12] A. Bonato, M. Lozier, D. Mitsche, X. Pérez-Giménez, P. Prałat, The domination number of on-line social networks and random geometric graphs, In: Proceedings of the 12th Conference on Theory and Applications of Models of Computation (TAMC'15), 2015.

[13] T.F. Coleman, J.J. Moré, Estimation of sparse Jacobian matrices and graph coloring problems, SIAM Journal on Numerical Analysis 20 (1983) 187-209.

[14] B. Corominas-Murtra, B. Fuches, S. Thurner, Detection of the elite structure in a virtual multiplex social system by means of a generalized K-core, Preprint 2015. 
[15] N.J. Cowan, E.J. Chastain, D.A. Vilhena, J.S. Freudenberg, C.T. Bergstrom, Nodal dynamics, not degree distributions, determine the structural controllability of complex networks, PLOS ONE 7 (2012) e38398.

[16] D. Crandall, D. Cosley, D. Huttenlocher, J. Kleinberg, S. Suri, Feedback effects between similarity and social influence in on-line communities, In: Proceedings of the 14th ACM SIGKDD Intl. Conf. on Knowledge Discovery and Data Mining, 2008.

[17] P. Erdős, A. Rényi, On random graphs I, Publicationes Mathematicae Debrecen 6 (1959) 290-297.

[18] E. Estrada, Spectral scaling and good expansion properties in complex networks, Europhysics Letters 73 (2006), 649-655.

[19] M.R. Garey, D.S. Johnson, Computers and intractability: a guide to theory of NP-completeness, W.H. Freeman \& Company, New York, 1979.

[20] S. Golder, D. Wilkinson, B. Huberman, Rhythms of social interaction: messaging within a massive on-line network, In: Proceedings of 3rd International Conference on Communities and Technologies, 2007.

[21] G.R. Grimmett, D.R. Stirzaker, Probability and Random Processes, Oxford University Press, 2001.

[22] S. Janson, T. Luczak, A. Rucinski, Random Graphs, Wiley-Interscience Series in Discrete Mathematics and Optimization, 2000.

[23] A. Java, X. Song, T. Finin, B. Tseng, Why we twitter: understanding microblogging usage and communities, In: Proceedings of the Joint 9th WEBKDD and 1st SNA-KDD Workshop 2007, 2007.

[24] A.D.I. Kramer, J.E. Guillory, J.T. Hancock, Experimental evidence of massive-scale emotional contagion through social networks, In: Proceedings of the National Academy of Sciences, 2014.

[25] R. Kumar, J. Novak, A. Tomkins, Structure and evolution of on-line social networks, In: In Proceedings of the 12th ACM SIGKDD International Conference on Knowledge Discovery and Data Mining, 2006.

[26] H. Kwak, C. Lee, H. Park, S. Moon, What is twitter, a social network or a news media?, In: Proceedings of the 19th International World Wide Web Conference, 2010.

[27] J. Leskovec, D. Chakrabarti, J. Kleinberg, C. Faloutsos, Realistic, mathematically tractable graph generation and evolution, using Kronecker multiplication, In: Proceedings of European Conference on Principles and Practice of Knowledge Discovery in Databases, 2005.

[28] J. Leskovec, J. Kleinberg, C. Faloutsos, Graphs over time: densification laws, shrinking diameters and possible explanations, In: Proceedings of the 13th ACM SIGKDD International Conference on Knowledge Discovery and Data Mining, 2005. 
[29] S. Lattanzi, D. Sivakumar, Affiliation Networks, In: In Proceedings of the 41st Annual ACM Symposium on Theory of Computing, 2009.

[30] M. McPherson, A Blau space primer: prolegomenon to an ecology of affiliation, In: Industrial and Corporate Change, Oxford University Press, 2004.

[31] J.M. McPherson, J.R. Ranger-Moore, Evolution on a dancing landscape: organizations and networks in dynamic Blau space, Social Forces (1991) 70 19-42.

[32] M. McPherson, L. Smith-John, J.M. Cook, Birds of a feather: Homophily in social networks, Annual Review of Sociology 27 (2001) 415-444.

[33] T. Milenković, V. Memišević, A. Bonato, N. Pržulj, Dominating biological networks, PLOS ONE 6 (2013) (8), e23016.

[34] S. Milgram, The small world problem, Psychology Today 2 (1967) 60-67.

[35] A. Mislove, M. Marcon, K. Gummadi, P. Druschel, B. Bhattacharjee, Measurement and analysis of on-line social networks, In: Proceedings of the 7th ACM SIGCOMM Conference on Internet Measurement, 2007.

[36] M.E.J. Newman, J. Park, Why social networks are different from other types of networks, Physical Review E 68(3) 036122 (2003).

[37] Ø. Ore, Theory of Graphs, American Mathematical Society Colloquium Publications, Providence, Rhode Island, 1962.

[38] V. Pareto, Cours d'Economie Politique, Geneva: Droz, 1896.

[39] M. Rai, S. Verma, S. Tapaswi, A power aware minimum connected dominating set for wireless sensor networks, Journal of networks 4 (2009) 511-519.

[40] I. Stojmenovic, M. Seddigh, J. Zunic, Dominating sets and neighbor elimination-based broadcasting algorithms in wireless networks, IEEE Transactions on Parallel and Distributed Systems 13 (2002) $14-25$.

[41] A.L. Traud, P.J. Mucha, M.A. Porter, Social structure of Facebook networks, Physica A: Statistical Mechanics and its Applications 391 (2012) 4165-4180.

[42] J.M.M. van Rooij, J. Nederlof, T.C. van Dijk, Inclusion/Exclusion Meets Measure and Conquer, Algorithms - ESA 20095757 (2009) 554-565.

[43] B. Viswanath, A. Mislove, M. Cha, K.P. Gummadi, On the evolution of user interaction in Facebook, In: Proceedings of the 2nd ACM SIGCOMM Workshop on Social Networks (WOSN'09), 2009.

[44] D.J. Watts, S. H., CollectiStrogatzve dynamics of 'small-world' networks, Nature 393 (1998) 440-442.

[45] J. Wu, M. Cardei, F. Dai, S. Yang, Extended Dominating Set and its Applications in Ad-Hoc Networks using Cooperative Communication, In: IEEE Transactions on Parallel and Distributed Systems, 2006. 